\title{
China Kidney Disease Network (CK-NET) 2015 Annual Data Report
}

\section{CHIEF EDITORS}

Luxia Zhang

Renal Division, Department of Medicine, Peking

University First Hospital; Peking University

Institute of Nephrology, Beijing, China; and Center

for Data Science in Health and Medicine, Peking

University, Beijing, China

\section{ASSOCIATE EDITORS}

Li Zuo

Department of Nephrology, Peking University

People's Hospital, Beijing, China

\section{Feng $\mathrm{Yu}$}

Renal Division, Department of Medicine, Peking

University First Hospital; Peking University

Institute of Nephrology, Beijing, China; and Blood

Purification Center of Nephrology Department,

Peking University International Hospital, Beijing,

China

Haibo Wang

Center for Data Science in Health and Medicine,

Peking University, Beijing, China; and China

Standard Medical Information Research Center,

Shenzhen, China

\section{CK-NET WORK GROUP (ALPHABETICALLY)}

Kunhao Bai

Department of Endoscopy, Sun Yat-sen University Cancer Center, State Key Laboratory of Oncology in South China, Collaborative Innovation Center for Cancer Medicine, Guangzhou, China

\section{Rui Chen}

Renal Division, Department of Medicine, Peking University First Hospital; Peking University Institute of Nephrology, Beijing, China

Hong Chu

Renal Division, Department of Medicine, Peking University First Hospital; Peking University Institute of Nephrology, Beijing, China

\section{Lanxia Gan}

China Standard Medical Information Research

Center, Shenzhen, China

Bixia Gao

Renal Division, Department of Medicine, Peking University First Hospital; Peking University Institute of Nephrology, Beijing, China
Ming-Hui Zhao

Renal Division, Department of Medicine, Peking

University First Hospital; Peking University

Institute of Nephrology, Beijing, China; and Peking-Tsinghua Center for Life Sciences, Beijing, China

Yue Wang

Department of Nephrology, Peking University

Third Hospital, Beijing, China

Hong Zhang

Renal Division, Department of Medicine, Peking University First Hospital; and Peking University Institute of Nephrology, Beijing, China

Xiangxiang $\mathrm{He}$

China Standard Medical Information Research Center, Shenzhen, China

Lili Liu

Renal Division, Department of Medicine, Peking University First Hospital; Peking University Institute of Nephrology, Beijing, China

Jianyan Long

Clinical Trial Unit, The First Affiliated Hospital of Sun Yat-sen University, Guangzhou, China

Ying Shi

China Standard Medical Information Research Center, Shenzhen, China

Zaiming Su

Center for Data Science in Health and Medicine, Peking University, Beijing, China

Xiaoyu Sun

Center for Data Science in Health and Medicine, Peking University, Beijing, China 


\section{Wen Tang}

Department of Nephrology, Peking University

Third Hospital, Beijing, China

Fang Wang

Renal Division, Department of Medicine, Peking University First Hospital; Peking University Institute of Nephrology, Beijing, China

Haibo Wang

China Standard Medical Information Research Center, Shenzhen, China; and Center for Data Science in Health and Medicine, Peking University, Beijing, China

Jinwei Wang

Renal Division, Department of Medicine, Peking University First Hospital; Peking University Institute of Nephrology, Beijing, China

\section{Song Wang}

Department of Nephrology, Peking University Third Hospital, Beijing, China

Yue Wang

Department of Nephrology, Peking University Third Hospital, Beijing, China

Chao Yang

Renal Division, Department of Medicine, Peking University First Hospital; Peking University Institute of Nephrology, Beijing, China

Feng Yu

Renal Division, Department of Medicine, Peking University First Hospital; Peking University Institute of Nephrology, Beijing, China; and Blood Purification Center of Nephrology Department, Peking University International Hospital, Beijing, China

\section{CK-NET INTERNATIONAL ADVISORY COMMITTEE (ALPHABETICALLY)}

Joseph Coresh

David Jayne

Andrew Levey

Vlado Perkovic

Rajiv Saran

\section{CK-NET DOMESTIC ADVISORY COMMITTEE (ALPHABETICALLY)}

Menghua Chen

Ping Fu

Guisen Li

Xinling Liang

Hongli Lin

Zhangsuo Liu
Dongliang Zhang

Blood Purification Center of Nephrology Department, Peking University International Hospital, Beijing, China

Hong Zhang

Renal Division, Department of Medicine, Peking University First Hospital; Peking University Institute of Nephrology, Beijing, China

Luxia Zhang

Renal Division, Department of Medicine, Peking University First Hospital; Peking University Institute of Nephrology, Beijing, China; and Center for Data Science in Health and Medicine, Peking University, Beijing, China

Minghui Zhao

Renal Division, Department of Medicine, Peking University First Hospital; Peking University Institute of Nephrology, Beijing, China; and PekingTsinghua Center for Life Sciences, Beijing, China

Xinju Zhao

Department of Nephrology, Peking University People's Hospital, Beijing, China

Liren Zheng

Blood Purification Center of Nephrology Department, Peking University International Hospital, Beijing, China

Zhiye Zhou

China Standard Medical Information Research Center, Shenzhen, China

Li Zuo

Department of Nephrology, Peking University People's Hospital, Beijing, China

Harold Feldman

Vivek Jha

Adeera Levin

Pierre Ronco

Sydney Tang

Jie Ding

Detian Li

Shaomei Li

Yunhua Liao

Jian Liu

Yingchun $\mathrm{Ma}$ 
Yonghui Mao

Caili Wang

Weiming Wang

Xiaoqin Wang

Zuying Xiong

Dongmei Xu

Xiaoping Yang

Yan Zha

Chun Zhang

Yiming Zhao

\section{CK-NET TECHNICAL ADVISORY COMMITTEE (ALPHABETICALLY)}

Kevin $\mathrm{He}$

Xiaohua Zhou
Luying Sun

Rong Wang

Wenke Wang

Changying Xing

Xudong Xu

Xiangdong Yang

Fan Yi

Aihua Zhang

Jinghong Zhao

Qiaoling Zhou

Guilan Kong 

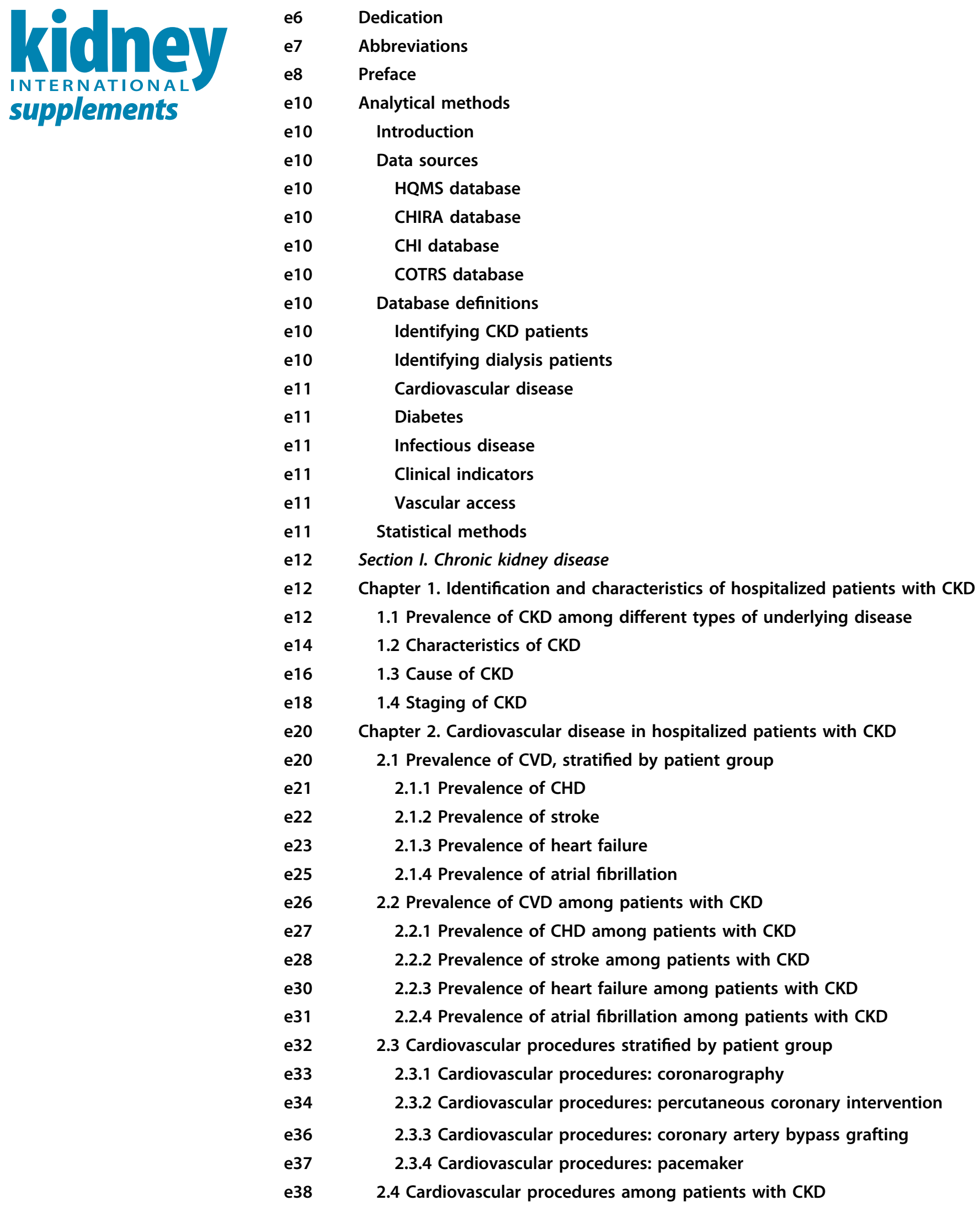
Chapter 3. Health care resource utilization of hospitalized patients with CKD 3.1 Costs

3.1.1 Overall medical care costs stratified by CKD, DM, and heart failure 3.1.2 Costs stratified by types of health insurance

3.1.3 Costs stratified by sex

3.1.4 Costs stratified by age

3.2 Length of stay

3.2.1 Overall length of stay stratified by CKD, DM, and heart failure

3.2.2 Length of stay stratified by types of health insurance

3.2.3 Length of stay stratified by sex

3.2.4 Length of stay stratified by age

Chapter 4. In-hospital mortality of hospitalized patients with CKD

4.1 In-hospital mortality rate stratified by CKD, DM, and heart failure

4.2 In-hospital mortality rate stratified by types of insurance

4.3 In-hospital mortality rate stratified by sex

4.4 In-hospital mortality rate stratified by age

Chapter 5. Acute kidney injury

5.1 Characteristics of AKI

5.1.1 Sex distribution of AKI, stratified by age

5.1.2 Age distribution of AKI, stratified by sex

5.2 Percentage of AKI

5.3 Percentage of CKD and DM among patients with AKI

Section II. End-stage kidney disease

Chapter 6. Prevalence, incidence, and characteristics of dialysis patients

Chapter 7. Clinical measurement and treatment among dialysis patients

Chapter 8. Vascular access

Chapter 9. Cardiovascular diseases and diabetes among dialysis patients

Chapter 10. Hospitalization

Chapter 11. Medical expenditures for dialysis patients

Chapter 12. Kidney transplant waiting list

Chapter 13. Discussion

References

Appendices: Definitions of ICD coding

Appendix 1 | Coding of various CKD etiologies

Appendix 2 | Coding of CKD stages

Appendix 3 | Coding of diabetes mellitus

Appendix 4 | Coding of hypertension

Appendix 5 | Coding of CVD

Appendix 6 | Coding of CVD operations

Appendix 7 | Coding of AKI 


\section{Dedication}

CHINA KIDNEY DISEASE NETWORK: A SWORD GRINDED WITH A DECADE'S DEDICATION

The China Kidney Disease Network (CK-NET), an initiative begun by the late Professor Hai-Yan Wang in 2014, is further developed in accordance with the national strategy of prompting big data application in China. Because surveillance systems for kidney disease are insufficient in China, CK-NET has provided resourceful information and benchmark data for kidney disease.

For China, a country with large population and vast territory, prompt and effective integration and mining of existing databases could be a promising strategy to improve the management of kidney disease. Further, the excellent paradigm CK-NET has set up can serve as a model for other diseases.

The establishment of CK-NET is the fruit of long-term preparation and unswerving effort. After the concept of chronic kidney disease (CKD) was proposed in 2002, epidemiological study of CKD soon started to be a hot topic all over the world. I am privileged to have the opportunity to bear witness to the important work and efforts of the team led by Professor Hai-Yan Wang over the past decade. Starting from a regional survey in Beijing in 2004 to a national multicenter cross-sectional study in 2012, and then to a largescale population cohort study in 2014, CK-NET has provided invaluable data regarding the disease burden of $\mathrm{CKD}$ in China, which are extremely valuable but scarce in other developing countries. As big data emerges in recent years, new methodologies and technologies have brought new opportunities for research on CKD at the population level. Against this backdrop, Professor Ming-Hui Zhao and
Professor Luxia Zhang have led the CK-NET team to embark on tremendous innovative and creative tasks, such as building a team composed of nephrologists, epidemiologists, and statisticians as well as setting up a platform for data integration and sharing, efforts that greatly promoted the application of big data in the field of nephrology.

The Annual Data Report (ADR) regarding kidney disease is the major output of CK-NET. The first ADR of CK-NET, which was published in 2017, focused on mostly pre-dialysis hospitalized patients in China. It is exciting to see that information regarding dialysis patients was included in this year's ADR. This is a gift that allows China and the rest of the world to have a comprehensive understanding of characteristics and trends of kidney disease with limited resources.

As the former president of the Chinese Society of Nephrology, it is my strong belief that CK-NET is a great initiative, and its contribution is a milestone for kidney disease management, prevention, and control. The ADR presented here shall provide nephrologists and health policy makers new insights into current the status of kidney disease in China. As depicted in an old Chinese poem, "10 years of effort it takes to grind this sword." CK-NET, facilitated by big data, is developing rapidly on the solid foundation laid by its predecessors.

Xue-Qing Yu, MD, PhD Former Chairman, Chinese Society of Nephrology Department of Nephrology Guangdong Provincial People's Hospital Guangdong Academy of Medical Science Guangzhou, Guangdong, China 


\section{Abbreviations}

\begin{tabular}{|c|c|c|c|}
\hline ADR & Annual Data Report & $\mathrm{HF}$ & heart failure \\
\hline $\mathrm{AF}$ & atrial fibrillation & HQMS & Hospital Quality Monitoring System \\
\hline AKI & acute kidney injury & $\mathrm{HT}$ & hypertension \\
\hline AMI & acute myocardial infarction & HTN & hypertensive nephropathy \\
\hline AVF & arteriovenous fistula & IBNR & incurred but not reported \\
\hline AVG & arteriovenous graft & ICD & International Classification of Diseases \\
\hline CABG & coronary artery bypass grafting & $\mathrm{ICU}$ & intensive care unit \\
\hline CAD & coronary artery disease & IQR & interquartile range \\
\hline CAG & coronarography & i.v. & intravenous \\
\hline $\mathrm{CHD}$ & coronary heart disease & LOS & length of stay \\
\hline $\mathrm{CHI}$ & commercial health insurance & $\mathrm{MBD}$ & mineral and bone disorder \\
\hline CHIRA & China Health Insurance Research & $\mathrm{NCC}$ & noncuffed catheter \\
\hline CKD & chronic kidney disease & NRCMS & new rural co-operative medical care \\
\hline CK-NET & China Kidney Disease Network & $\mathrm{ON}$ & obstructive nephropathy \\
\hline COTRS & China Organ Transplant Response System & PAD & peripheral arterial disease \\
\hline CTIN & chronic tubulointerstitial nephritis & PCI & percutaneous coronary intervention \\
\hline CVA & cerebrovascular accident & $\mathrm{PD}$ & peritoneal dialysis \\
\hline CVC & central venous catheter & PMP & per million population \\
\hline CVD & cardiovascular disease & PPPY & per person per year \\
\hline DKD & diabetic kidney disease & PTH & parathyroid hormone \\
\hline DM & diabetes mellitus & $\mathrm{RMB}$ & renminbi \\
\hline $\mathrm{EPO}$ & erythropoietin & SD & standard deviation \\
\hline ESKD & end-stage kidney disease & TCC & tunneled cuffed catheter \\
\hline FFBI & Fistula First Breakthrough Initiative & TIA & transient ischemic attack \\
\hline GN & CKD due to glomerulonephritis & UBMI & urban basic medical insurance \\
\hline HbA1c & hemoglobin A1c & USRDS & United States Renal Data System \\
\hline $\mathrm{HD}$ & hemodialysis & VA & vascular access \\
\hline
\end{tabular}




\section{Preface}

Chronic kidney disease (CKD) has received increased attention as a leading public health problem worldwide. Along with the rapid growth of economy and gradual changes of lifestyle, China is facing an ever-increasing burden of CKD. However, the lack of a national surveillance system for kidney disease and unmet need for nephrology research in China have become an obstacle to develop effective preventive strategies.

The emergence and development of big data provided a unique opportunity to address the burden of CKD in China. After long-term planning, China Kidney Disease Network (CK-NET) was founded based on previous nationwide epidemiological studies regarding kidney disease led by Peking University First Hospital and the collaboration network involving 59 large renal centers in China (Figure 1). The mission of CK-NET is, by integrating various sources of data involving kidney disease in China and utilizing cuttingedge data analytics, to provide data-based evidence for health care policy, strengthen academic research, and promote effective disease management in the field of nephrology.

In 2017, CK-NET released its first publication of an Annual Data Report (ADR), which provided useful and substantial information regarding CKD. It was published in a supplement to the American Journal of Kidney Diseases. ${ }^{1}$ This year marks the second publication of a CK-NET ADR. Based on the Hospital Quality Monitoring System (HQMS), which is also the data source of the first ADR, this year's ADR describes 887,816 hospitalized patients with CKD in 2015. Compared with the first ADR, contents that are less relevant to international readers have been deleted and certain parts (especially the cardiovascular chapter) have been enriched in the second ADR. Acute kidney injury, as an important topic, is described in a separate chapter.

Furthermore, 2 more nationwide claims databases, the China Health Insurance Research (CHIRA) database and commercial health insurance $(\mathrm{CHI})$ database, were included

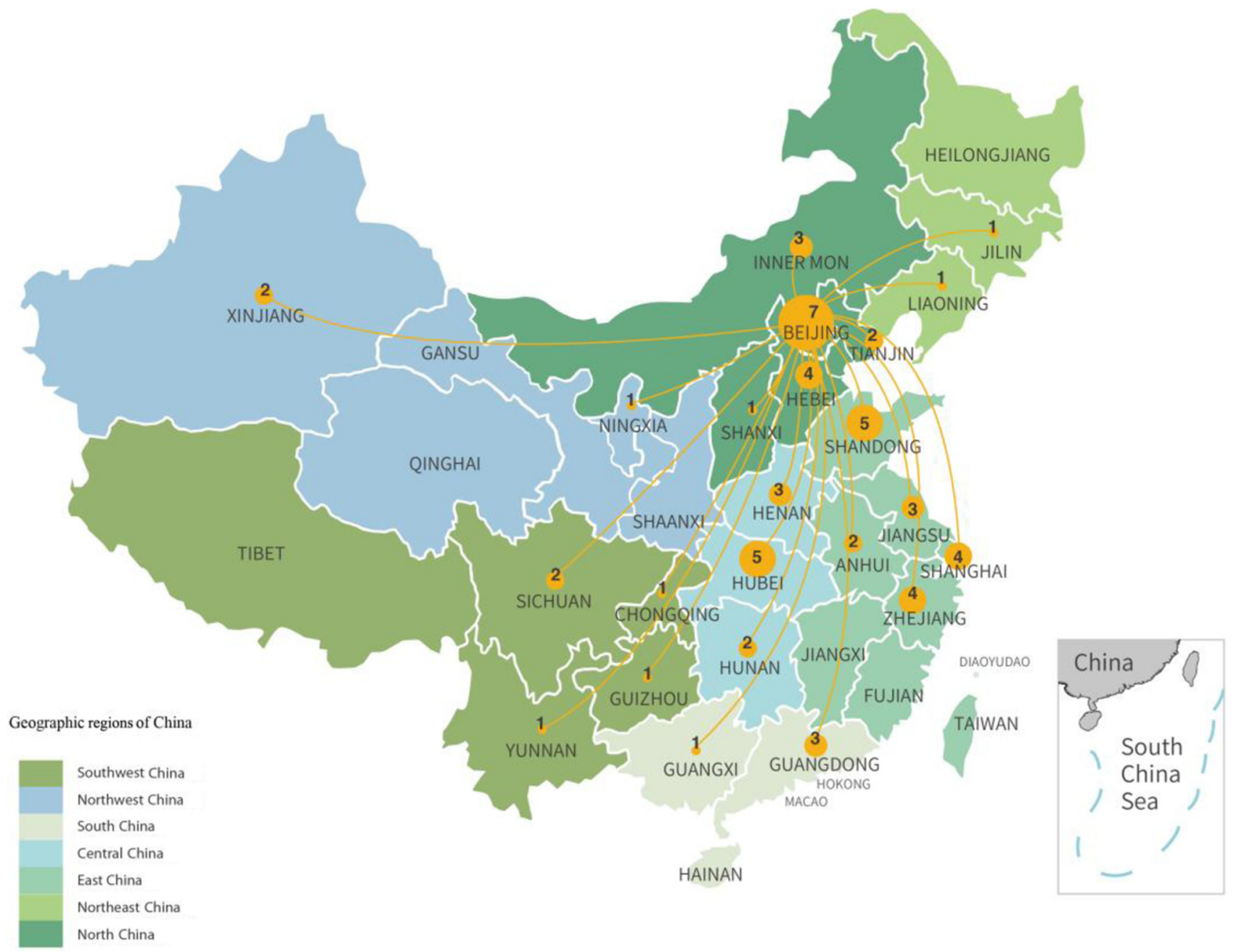

Figure 1| Geographical distribution of China Kidney Disease Network (CK-NET) collaborating renal centers in China. The size of each yellow circle and its corresponding number indicate the number of collaborating renal centers in the city or province. 
and provided information regarding dialysis in China, which enriched the contents of the ADR substantially. Seven chapters were included to provide a better understanding of endstage kidney disease (ESKD) in China.

The strengths of the second CK-NET ADR include a large patient sample size, stringent data quality control during data collection, and maximum utilization of multiple-source data. However, several points should be considered when interpreting the results in the second CK-NET ADR. First, selection bias cannot be ruled out because of limitations in data sampling. Second, International Classification of Diseases (ICD) coding is used to define CKD and certain other diseases likely with low sensitivity and high specificity, which may be influenced by various diagnostic criteria and coding procedures. Finally, the current ADR includes only cross-sectional data, making causal inference difficult. A brief interpretation is included in each chapter to facilitate understanding of the contents.

The second CK-NET ADR symbolizes a successful research effort in the era of big data. It uses the platform built by the
National Health Commission of the People's Republic of China, with support from the specialists of Peking University and its affiliated hospitals. The innovative collaborative model will bring inspiration and substantial changes to health service and medical research in China.

\section{DISCLOSURE}

Publication of this article was supported by Peking University. All the authors declared no competing interests.

\section{ACKNOWLEDGMENTS}

We thank the National Health Commission of China, China Health Insurance Research Association, China Organ Transplantation Development Foundation, Peking University, and China Standard Medical Information Research Center for support of this study. We thank CK-NET collaborating centers, members, and volunteers for their hard work and efforts. We thank every participant who has contributed important and invaluable data to CK-NET. 


\section{Analytical methods}

\section{INTRODUCTION}

The analytical methods chapter describes the data sources, database definition, and analytical methods of the second ADR. For this ADR, we report on data from January 1, 2015, to December 31, 2015. Only data from adult patients (aged 18 years or older) are included. The analyses are based on 4 national databases: the Hospital Quality Monitoring System (HQMS) database, China Health Insurance Research (CHIRA) database, commercial health insurance $(\mathrm{CHI})$ database, and China Organ Transplant Response System (COTRS) database.

The ethics committee of Peking University First Hospital approved this study. The contents of this report have been internally and externally reviewed and submitted to the National Health Commission of the People's Republic of China. The statistical analyses were performed using Microsoft Excel 2016 (Microsoft Corp., Redmond, WA) and SAS 9.4 (SAS Institute Inc., Cary, NC).

\section{DATA SOURCES}

HQMS database. HQMS is a mandatory national inpatient database system under the authority of the National Health Commission of the People's Republic of China. All tertiary hospitals in China have been requested to submit standardized inpatient discharge records to HQMS daily in an automated manner since 2013. Tertiary hospitals constitute the top tier of the medical system in China; at a minimum, they must have 500 beds and accreditation from health authorities. As opposed to tertiary hospitals in the Western medical system, tertiary hospitals in China provide primary, secondary, and tertiary care and have exposure to a nationwide patient population.

Patient-level data were collected from the nationally uniform front page of the hospitalization medical record. Altogether 353 variables including patient demographics, clinical diagnosis (ICD10 ), procedures and operations, financial breakdowns, and information of affiliated hospitals or divisions were collected. As a part of stringent standard practice in China, the front page has legal validity and must be completed by the caregiving doctors who have the most accurate and comprehensive understanding of the patient's medical condition. The diagnoses were then coded according to the ICD-10 coding system by certified professional medical coders at each hospital. The HQMS data reporting system performs automated data quality control on a daily basis at the time of data submission to ensure the completeness, consistency, and accuracy of data. If inconsistencies are detected, the entire daily data package of the hospital will be rejected and the hospital will be required to review and resubmit data. As of December 2015, the HQMS database automated data exchange network covered more than 900 tertiary hospitals in 31 provinces, and had collected over 40 million inpatient records.

CHIRA database. Urban basic medical insurance (UBMI) is the predominant medical insurance program in urban areas of China, covering 31 provinces and municipalities (Hong Kong, Macao, and Taiwan are not included). UBMI comprises the Urban Employee Basic Health Insurance and the Urban Resident Basic Health Insurance. By 2015, the number of insured people reached 600 million and 377 million, respectively.

CHIRA is an administrative database initiated in 2007 and managed by the China Health Insurance Research Association. Information on every hospital visit and medical service claim was recorded in the database. Primary diagnosis, patient demographics, frequency of laboratory tests, prescription drugs, operation procedures, and medical expenditures were also recorded. The CHIRA data covered not only inpatients' information, but also that of outpatients. All medical information was recorded at each hospital grade and was not limited to tertiary hospitals. A 2-stage sampling design was used to draw this national sample of the UBMI beneficiaries in 30 provinces, autonomous regions, and municipalities directly under the central government (excluding Ningxia, Hong Kong, Macao, and Taiwan). In the first stage, convenience sampling was performed on 4 municipalities directly under the central government (Beijing, Shanghai, Tianjin, and Chongqing), 26 provincial capital cities, and a certain number of prefecture cities. In the second stage, a systematic random sampling sorting by age was used to extract $2 \%$ of the beneficiaries from those of the municipalities and provincial capital cities, and 5\% from those of prefecture-level cities. The number of sampling beneficiaries in the CHIRA database in 2015 was $6,736,536$, with their whole-year medical records recorded. All personal information was anonymized and de-identified prior to analysis for privacy protection reasons.

CHI database. $\mathrm{CHI}$ is a database from the 6 top commercial insurance companies with the largest market share in mainland China, covering 22 kinds of major disease insurance and over 60 million customers in 2015. The number of insurance policies exceeded 95 million from 1995 to 2016 in 31 provinces, autonomous regions, and municipalities directly under the central government (excluding Hong Kong, Macao, and Taiwan). Information of sex, age, insured amount, region, occupation, income, and disease diagnosis of policy holders was recorded in the database. People aged 0 to 4 years and 25 to 49 years were the core insured population, with an average age of 30.9 years. Dialysis incidence and mortality of patients receiving dialysis were analyzed based on the $\mathrm{CHI}$ database.

COTRS database. As of September 1, 2013, it became mandatory to allocate organs through COTRS, which is a national open and transparent organ allocation computer system. COTRS is maintained by an impartial third party. The matching of donor organs to recipients initially includes medical emergency status, time on waiting list, and histocompatibility. The chapter about waiting lists for kidney transplantation in China was based on analysis of the COTRS database.

\section{DATABASE DEFINITIONS}

Identifying CKD patients. Three sets of ICD-10 disease codes are used to identify patients with CKD in tertiary hospitals in China based on the HQMS database: Beijing version 4.0, national standard version 1.0, and national clinical version 1.0. Codes for procedures and operations are based on the Beijing version and national clinical version. Patients with diabetic kidney disease were defined as those with both diabetes and CKD, and without reported causes of CKD other than diabetic kidney disease. ${ }^{2-4}$ Results of renal biopsy were not available for most of them. Despite being aware of that acute kidney injury might be substantially underestimated by ICD-10 coding, we retained the chapter because it could reflect the reality of diagnoses. All relevant ICD codes are listed in Appendices 1 through 7.

Identifying dialysis patients. Dialysis patients were identified according to the items of medical service claim and ICD-10 codes, which was defined as CKD requiring dialysis (hemodialysis [HD] and peritoneal dialysis $[\mathrm{PD}]$ ), excluding acute renal failure. PD patients were identified by claim records of peritoneal dialysis fluid, and HD patients were identified by related claim records of HD. 
Cardiovascular disease. Patients with cardiovascular disease (CVD) were identified by the diagnosis of CVD (ICD-10 coding), claim records of therapeutic drugs for CVD based on the Anatomical Therapeutic Chemical (ATC) codes (C01, cardiac therapy), and related surgical procedures, such as coronary artery computed tomography and coronary arteriography. Coronary heart disease, acute myocardial infarction, heart failure, cerebrovascular accident or transient ischemic attack, peripheral arterial disease, atrial fibrillation, and cardiovascular procedures (percutaneous coronary intervention and pacemaker) were also identified by ICD-10 coding and related claim records.

Diabetes. Patients with diabetes were identified by the diagnosis of diabetes (ICD-10 coding) and claims records of therapeutic drugs for diabetes (A10, drugs used in diabetes). The subgroup of patients with diabetes in the results did not necessarily have kidney disease.

Infectious disease. Infectious disease was identified by the top 3 ICD-10 coding of infection by various pathogens.

Clinical indicators. Laboratory tests and drug usage were identified by claim records. Laboratory tests include blood hemoglobin, serum levels of iron, total calcium, phosphorus, parathyroid hormone (PTH), albumin, lipids and hemoglobin Alc, and fundus examination to detect diabetic retinopathy. Drug usage included erythropoietin, iron (i.v. iron and oral iron), calcitriol, phosphate binder, and transfusion therapy.

Vascular access. The definitions of tunneled and cuffed catheter (TCC), noncuffed catheter (NCC), interventions for autogenous arteriovenous fistula (AVF) and/or autogenous arteriovenous graft (AVG), and stable AVF/AVG for HD patients were based on the claim records of surgical interventions, medical materials, nursing treatments, and so on. For PD, the identification of newly inserted peritoneal catheters, transient central venous catheters (CVC), and stable patients was conducted in the same way.

\section{STATISTICAL METHODS}

Statistical methods include descriptive statistics, such as frequency, percentage, median with interquartile range, mean, and SD. The results were generally described by sex, age, geographic distribution, comorbidity, and dialysis modality. $P$ values were not included, due to large sample sizes.

The comparisons between the 2 groups of patients with diabetes and those with CKD were based on the overall population, respectively, which meant we did not exclude diabetes patients also having CKD or CKD patients also having diabetes. The prevalence of dialysis was estimated by multiplying the percentage of dialysis patients in sampled data from the CHIRA database in different geographic areas and the relevant UBMI utilization rate (partial data were from the 2017 China Health Statistics Yearbook). The incidence count in the $\mathrm{CHI}$ database has taken into account incurred but not reported (IBNR) events, which were often used to estimate the corresponding incidence rates in insurance industries. The adjusted incidence of dialysis was standardized by the direct method using the 2010 national population census data.

In the scenario that the interval between hospital discharge and following readmission was less than 3 days, we considered it as a continuous hospitalization. One hospitalization with a length of stay $\geq$ 180 days was excluded. In the chapter on vascular access, HD patients would belong to only 1 group by a certain filter sequence from operational AVF/AVG to TCC to NCC. If more than 1 kind of intervention was performed, the anterior filter situation would be selected. Patients without any intervention would be recognized as belonging to stable the AVF/AVG group. We could not distinguish AVF from AVG in the present database. PD patients would belong to only 1 group by the same method. The first selected group was newly inserted PD catheter, and the second selected one was transient CVC. The other patients belonged to the stable PD group. We did not further separate TCC and NCC in the CVC group, because TCC was seldom used. 


\title{
Section I. Chronic kidney disease
}

\section{Chapter 1: Identification and characteristics of hospitalized patients with CKD}

\author{
Fang Wang ${ }^{1}$, Jianyan Long $^{2}$, Kunhao Bai ${ }^{3}$, Bixia Gao ${ }^{1}$, Lanxia Gan ${ }^{4}$, Chao Yang ${ }^{1}$ and Haibo Wang ${ }^{4,5}$ \\ ${ }^{1}$ Renal Division, Department of Medicine, Peking University First Hospital; Peking University Institute of Nephrology, Beijing, China; \\ ${ }^{2}$ Clinical Trial Unit, The First Affiliated Hospital of Sun Yat-sen University, Guangzhou, China; ${ }^{3}$ Department of Endoscopy, Sun Yat- \\ sen University Cancer Center, State Key Laboratory of Oncology in South China, Collaborative Innovation Center for Cancer \\ Medicine, Guangzhou, China; ${ }^{4}$ China Standard Medical Information Research Center, Shenzhen, China; and ${ }^{5}$ Center for Data \\ Science in Health and Medicine, Peking University, Beijing, China
}

This chapter focuses on the prevalence, characteristics, and etiology of patients with CKD in the hospitalized population, aiming to provide an overall description of the disease.

Patients with CKD constituted $4.80 \%$ of all inpatients, and the percentage was higher among those with other noncommunicable chronic diseases (especially diabetes) (Figure 2 and Table 1). The percentage increased with age gradually, and reached $9.69 \%$ for those aged 85 years or older (Figure 3 and Table 2). However, it should be noted that percentages reflected both the actual prevalence and the diagnosis rate (Figure 4, Table 3).

For patients with $\mathrm{CKD}$, a male predominance was observed (58.93\%), and it was consistent across age groups (Figure 5 and Table 4). Nearly one-half of the patients with CKD were aged 60 years or older (Figure 6 and Table 5).

Regarding the etiology of CKD, the most common causes included diabetic kidney disease (DKD, 26.96\%), hypertensive nephropathy (HTN, 20.78\%), obstructive nephropathy
(ON, 15.59\%), and glomerulonephritis (GN, 15.07\%) (Figure 7 and Table 6). The spectrum differed between urban and rural residents. For urban residents, over one-half of the patients were diagnosed with DKD (32.73\%) or HTN (22.98\%). For rural residents, the top 3 causes were ON (21.41\%), GN (18.53\%), and DKD (17.36\%) (Figure 8 and Table 7). Geographic variation of etiology was also observed, which showed a relatively high percentage of DKD in Northern China and high percentage of ON in Southern China (Figure 9 and Table 8).

Finally, although it is clear that CKD staging is an important part of the diagnosis in both the Kidney Disease Outcomes Quality Initiative (2002) and the Kidney Disease: Improving Global Outcomes (2012) guidelines, only $15.08 \%$ of inpatients with CKD in the dataset had ICD-10 codes for CKD staging. Among these, $14.85 \%$ and $49.27 \%$ had CKD stages 4 and 5 , respectively, reflecting the pattern of diagnosis rather than the pattern of disease (Figure 10 and Table 9).

\subsection{Prevalence of CKD among different types of underlying disease}

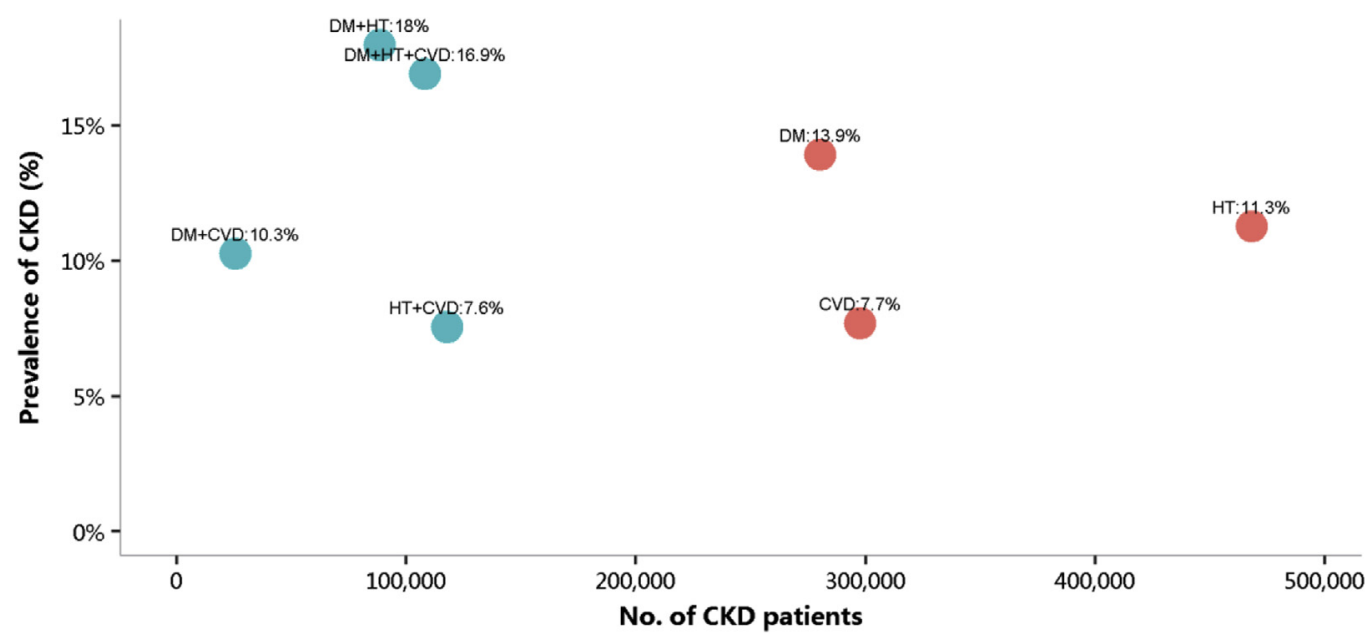

Figure 2 | Prevalence of CKD among different types of underlying disease. CKD, chronic kidney disease; CVD, cardiovascular disease; DM, diabetes mellitus; HT, hypertension. 
Table 1 | Prevalence of CKD among different types of underlying disease, $N(\%)$

\begin{tabular}{|c|c|c|}
\hline Patient group & No. of CKD patients & Prevalence of CKD (\%) \\
\hline HQMS & 887,816 & 4.80 \\
\hline HT & 468,014 & 11.29 \\
\hline CVD & 297,434 & 7.70 \\
\hline DM & 280,107 & 13.92 \\
\hline $\mathrm{HT}+\mathrm{CVD}$ & 117,587 & 7.57 \\
\hline $\mathrm{DM}+\mathrm{HT}+\mathrm{CVD}$ & 108,134 & 16.90 \\
\hline $\mathrm{DM}+\mathrm{HT}$ & 88,455 & 17.98 \\
\hline $\mathrm{DM}+\mathrm{CVD}$ & 25,585 & 10.27 \\
\hline
\end{tabular}

CKD, chronic kidney disease; CVD, cardiovascular disease; DM, diabetes mellitus; HQMS, Hospital Quality Monitoring System; HT, hypertension.

HQMS, adult patients (age $\geq 18$ years) in HQMS dataset. HT, hypertension: I10-I13, I15; DM, diabetes mellitus: E10-E14; CKD, chronic kidney disease (refer to Appendix 1); CVD, cardiovascular disease (refer to Appendix 5).

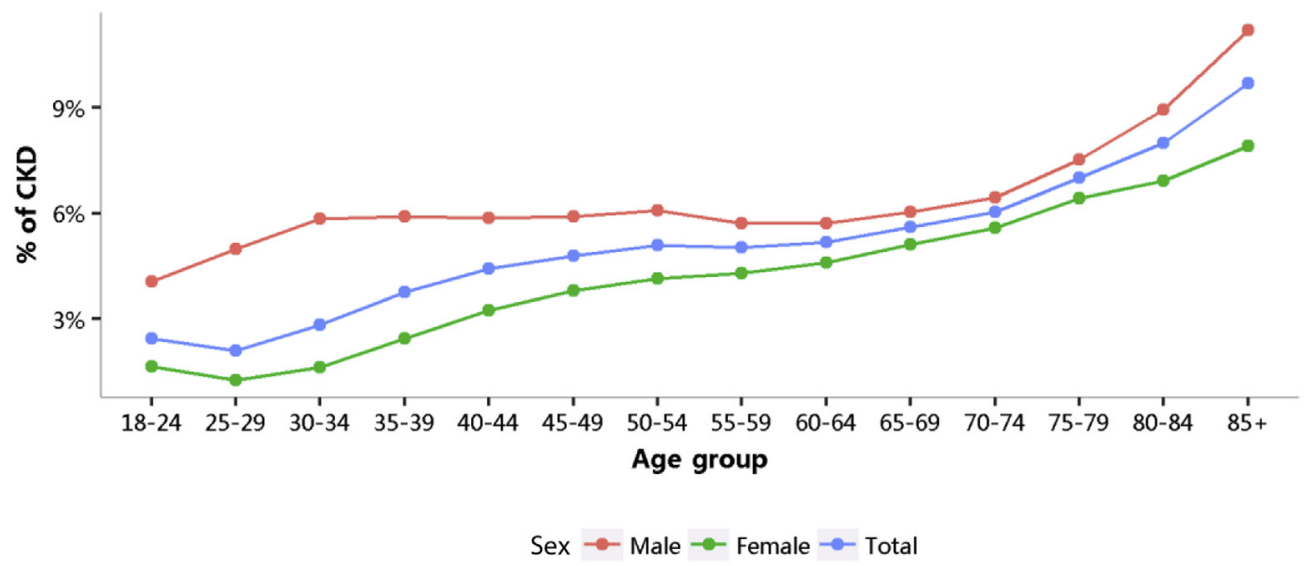

Figure 3 | Patients with CKD, stratified by sex and age group. CKD, chronic kidney disease.

Table 2 | Patients with CKD, stratified by sex and age group, $N$ (\%)

\begin{tabular}{lcrr}
\hline Age group & Male & Female & Total \\
\hline $18-24$ & $13,369(4.06)$ & $10,983(1.65)$ & $24,352(2.45)$ \\
$25-29$ & $17,774(4.99)$ & $15,907(1.28)$ & $33,681(2.11)$ \\
$30-34$ & $20,705(5.83)$ & $14,797(1.64)$ & $35,502(2.83)$ \\
$35-39$ & $24,536(5.90)$ & $16,715(2.45)$ & $41,251(3.76)$ \\
$40-44$ & $35,961(5.86)$ & $24,701(3.26)$ & $60,662(4.42)$ \\
$45-49$ & $45,687(5.90)$ & $33,259(3.80)$ & $78,946(4.79)$ \\
$50-54$ & $53,428(6.09)$ & $38,172(4.14)$ & $91,600(5.09)$ \\
$55-59$ & $50,999(5.71)$ & $35,984(4.29)$ & $86,983(5.02)$ \\
$60-64$ & $58,827(5.72)$ & $43,282(4.59)$ & $102,109(5.18)$ \\
$65-69$ & $51,873(6.04)$ & $38,718(5.12)$ & $90,591(5.61)$ \\
$70-74$ & $43,999(6.45)$ & $33,749(5.58)$ & $77,748(6.04)$ \\
$75-79$ & $42,811(7.51)$ & $32,733(6.43)$ & $75,544(7.00)$ \\
$80-84$ & $32,852(8.93)$ & $21,874(6.92)$ & $54,726(8.00)$ \\
$85+$ & $21,470(11.18)$ & $12,651(7.90)$ & $34,121(9.69)$ \\
Total & $514,291(6.19)$ & $373,525(3.67)$ & $887,816(4.80)$ \\
\hline
\end{tabular}

CKD, chronic kidney disease. 


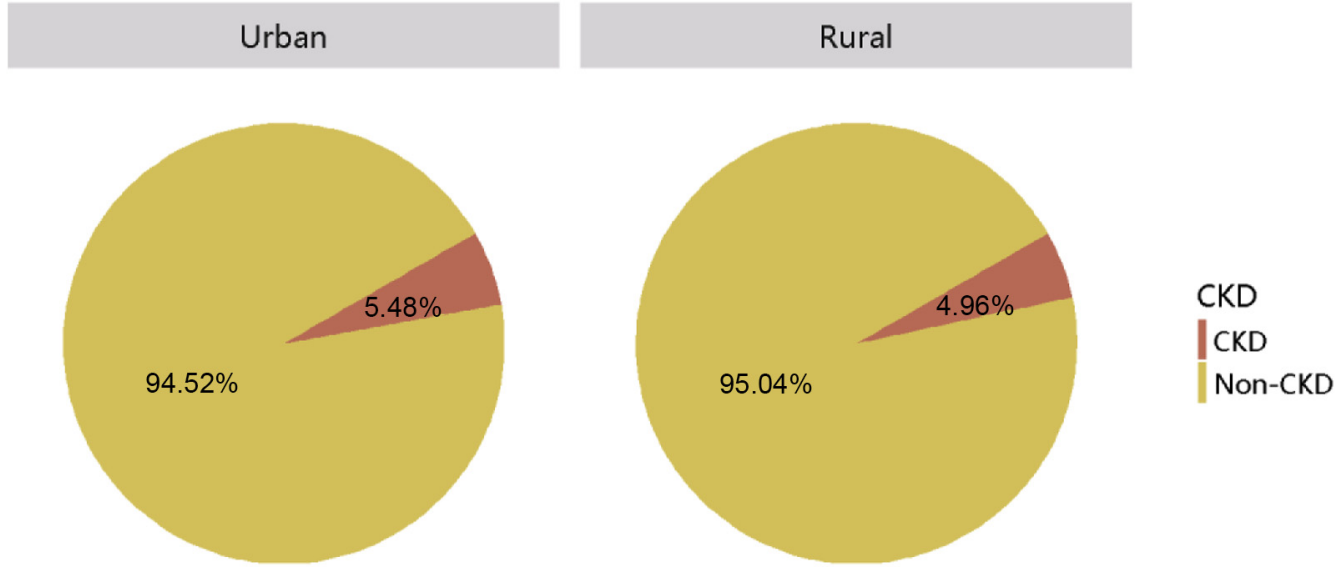

Figure 4| Patients with CKD, stratified by urban versus rural area. CKD, chronic kidney disease.

Table 3 | Patients with CKD, stratified by urban versus rural area, $N(\%)$

\begin{tabular}{lr}
\hline Residence & CKD \\
\hline Urban & $454,841(5.48)$ \\
Rural & $189,785(4.96)$ \\
Total & $644,626(5.31)$ \\
\hline
\end{tabular}

CKD, chronic kidney disease.

Patients with missing data for residence were not included in the analysis. HQMS: 6,360,952 (34.40\%).

\subsection{Characteristics of CKD}

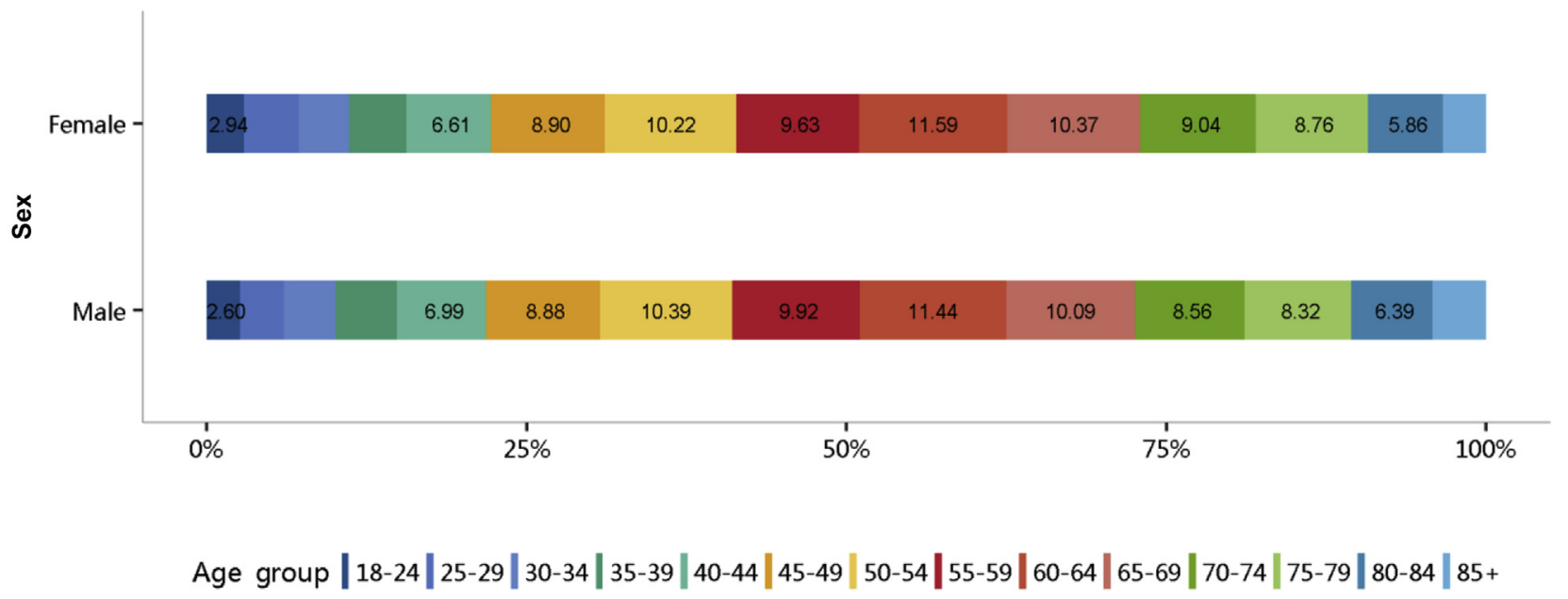

Figure 5| Age distribution of CKD, stratified by sex. CKD, chronic kidney disease. 
Table 4| Age distribution of CKD, stratified by sex, $N$ (\%)

\begin{tabular}{|c|c|c|c|}
\hline Age group & Male & Female & Total \\
\hline $18-24$ & $13,369(2.60)$ & $10,983(2.94)$ & $24,352(2.74)$ \\
\hline $25-29$ & $17,774(3.46)$ & $15,907(4.26)$ & 33,681 (3.79) \\
\hline $30-34$ & $20,705(4.03)$ & 14,797 (3.96) & $35,502(4.00)$ \\
\hline $35-39$ & 24,536 (4.77) & 16,715 (4.47) & 41,251 (4.65) \\
\hline $40-44$ & 35,961 (6.99) & $24,701(6.61)$ & $60,662(6.83)$ \\
\hline $45-49$ & $45,687(8.88)$ & $33,259(8.90)$ & 78,946 (8.89) \\
\hline $50-54$ & $53,428(10.39)$ & $38,172(10.22)$ & $91,600(10.32)$ \\
\hline $55-59$ & 50,999 (9.92) & $35,984(9.63)$ & $86,983(9.80)$ \\
\hline $60-64$ & $58,827(11.44)$ & 43,282 (11.59) & 102,109 (11.50) \\
\hline $65-69$ & $51,873(10.09)$ & $38,718(10.37)$ & $90,591(10.20)$ \\
\hline $70-74$ & $43,999(8.56)$ & $33,749(9.04)$ & $77,748(8.76)$ \\
\hline $75-79$ & $42,811(8.32)$ & $32,733(8.76)$ & $75,544(8.51)$ \\
\hline $80-84$ & 32,852 (6.39) & $21,874(5.86)$ & $54,726(6.16)$ \\
\hline $85+$ & $21,470(4.17)$ & 12,651 (3.39) & $34,121(3.84)$ \\
\hline Total & 514,291 & 373,525 & 887,816 \\
\hline
\end{tabular}

CKD, chronic kidney disease.

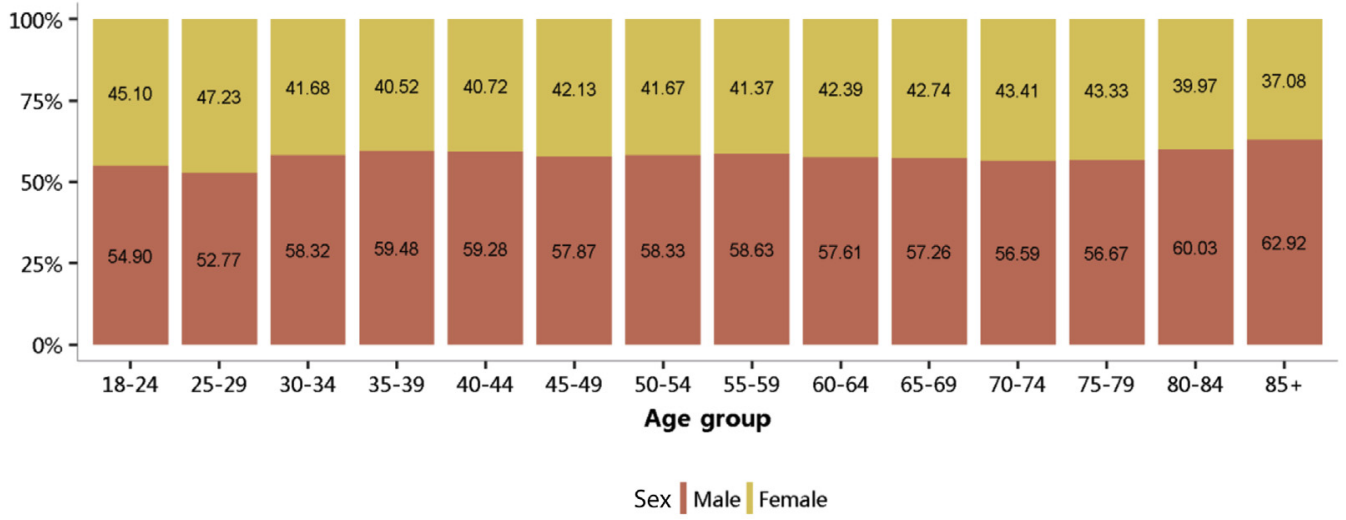

Figure 6 | Sex distribution of CKD, stratified by age. CKD, chronic kidney disease.

Table 5 | Sex distribution of CKD, stratified by age, $N$ (\%)

\begin{tabular}{|c|c|c|c|}
\hline Age group & Male & Female & Total \\
\hline $18-24$ & $13,369(54.90)$ & $10,983(45.10)$ & 24,352 \\
\hline $25-29$ & $17,774(52.77)$ & $15,907(47.23)$ & 33,681 \\
\hline $30-34$ & $20,705(58.32)$ & $14,797(41.68)$ & 35,502 \\
\hline $40-44$ & 35,961 (59.28) & $24,701(40.72)$ & 60,662 \\
\hline $45-49$ & $45,687(57.87)$ & $33,259(42.13)$ & 78,946 \\
\hline $50-54$ & $53,428(58.33)$ & $38,172(41.67)$ & 91,600 \\
\hline $65-69$ & $51,873(57.26)$ & $38,718(42.74)$ & 90,591 \\
\hline $70-74$ & $43,999(56.59)$ & $33,749(43.41)$ & 77,748 \\
\hline $75-79$ & $42,811(56.67)$ & $32,733(43.33)$ & 75,544 \\
\hline $80-84$ & $32,852(60.03)$ & $21,874(39.97)$ & 54,726 \\
\hline $85+$ & $21,470(62.92)$ & $12,651(37.08)$ & 34,121 \\
\hline Total & $514,291(57.93)$ & $373,525(42.07)$ & 887,816 \\
\hline
\end{tabular}

CKD, chronic kidney disease. 


\subsection{Cause of CKD}

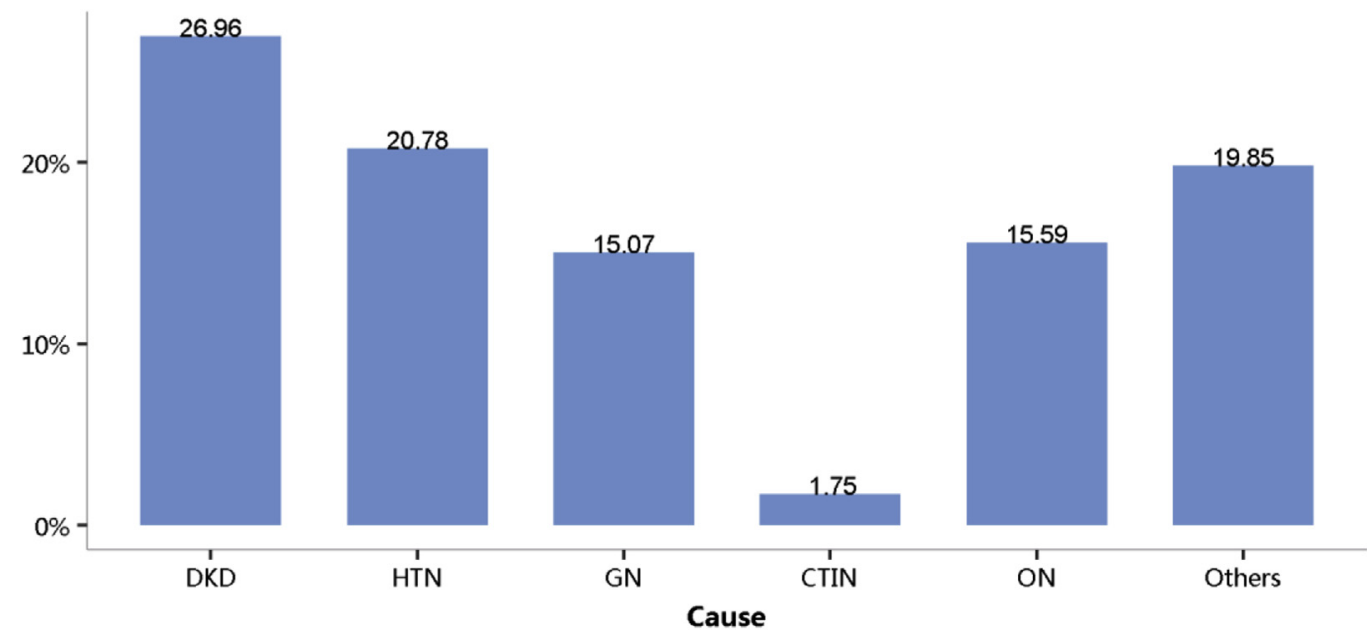

Figure 7| Cause distribution of CKD. CKD, chronic kidney disease; CTIN, chronic tubulointerstitial nephritis; DKD, diabetic kidney disease; GN, CKD due to glomerulonephritis; HTN, hypertensive nephropathy; ON, obstructive nephropathy; Others, CKD due to other reasons.

Table 6 | Cause distribution of CKD

\begin{tabular}{lr}
\hline Cause & $N(\%)$ \\
\hline DKD & $239,354(26.96)$ \\
HTN & $184,486(20.78)$ \\
GN & $133,822(15.07)$ \\
CTIN & $15,524(1.75)$ \\
ON & $138,378(15.59)$ \\
Others & $176,252(19.85)$ \\
Total & 887,816 \\
\hline
\end{tabular}

CKD, chronic kidney disease; CTIN, chronic tubulointerstitial nephritis; DKD, diabetic kidney disease; GN, CKD due to glomerulonephritis; HTN, hypertensive nephropathy; ON, obstructive nephropathy; Others, CKD due to other reasons.

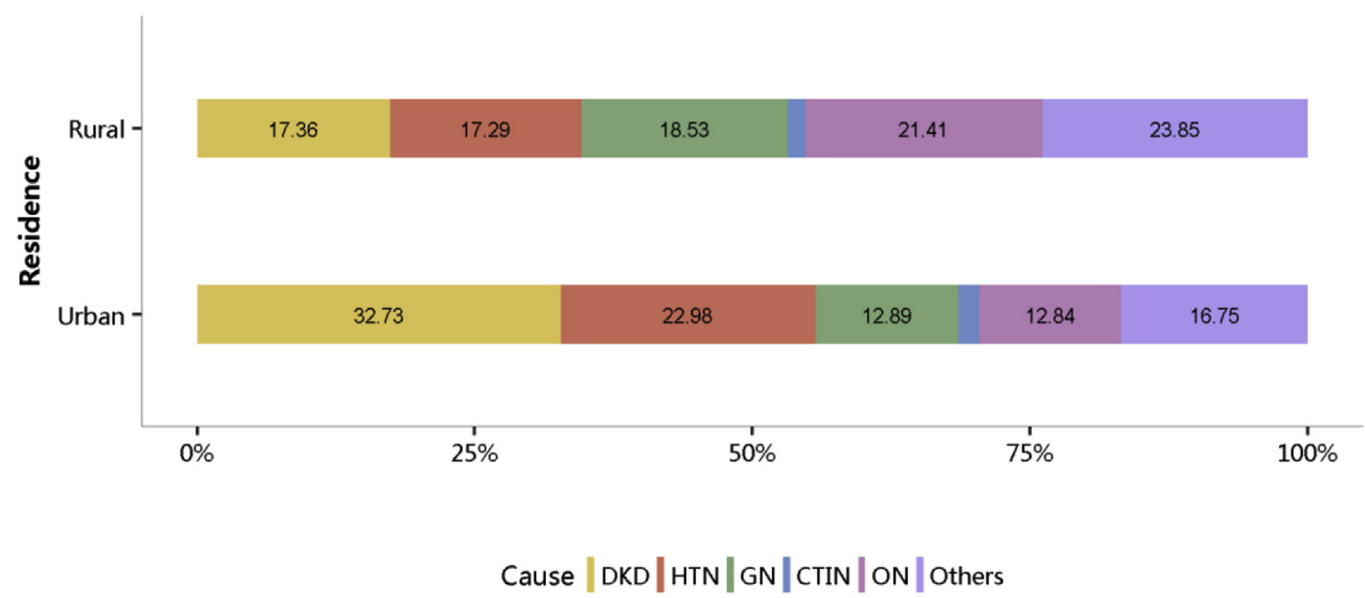

Figure 8 | Cause of CKD, stratified by urban versus rural area. CKD, chronic kidney disease; CTIN, chronic tubulointerstitial nephritis; DKD, diabetic kidney disease; GN, CKD due to glomerulonephritis; HTN, hypertensive nephropathy; ON, obstructive nephropathy; Others, CKD due to other reasons. 
Table 7| Cause of CKD, stratified by urban versus rural area

\begin{tabular}{llll}
\hline Cause & \multicolumn{1}{c}{ Urban } & \multicolumn{1}{c}{ Rural } & Total \\
\hline DKD & $148,848(32.73)$ & $32,944(17.36)$ & $181,792(28.20)$ \\
HTN & $104,531(22.98)$ & $32,814(17.29)$ & $137,345(21.31)$ \\
GN & $58,636(12.89)$ & $35,158(18.53)$ & $93,794(14.55)$ \\
CTIN & $8247(1.81)$ & $2975(1.57)$ & $11,222(1.74)$ \\
ON & $58,414(12.84)$ & $40,627(21.41)$ & $99,041(15.36)$ \\
Others & $76,165(16.75)$ & $45,267(23.85)$ & $121,432(18.84)$ \\
Total & 454,841 & 189,785 & 644,626 \\
\hline
\end{tabular}

CKD, chronic kidney disease; CTIN, chronic tubulointerstitial nephritis; DKD, diabetic kidney disease; GN, CKD due to glomerulonephritis; HTN, hypertensive nephropathy; ON, obstructive nephropathy; Others, CKD due to other reasons.

Patients with missing data for residence were not included in the analysis. CKD: 243,190 (27.39\%).

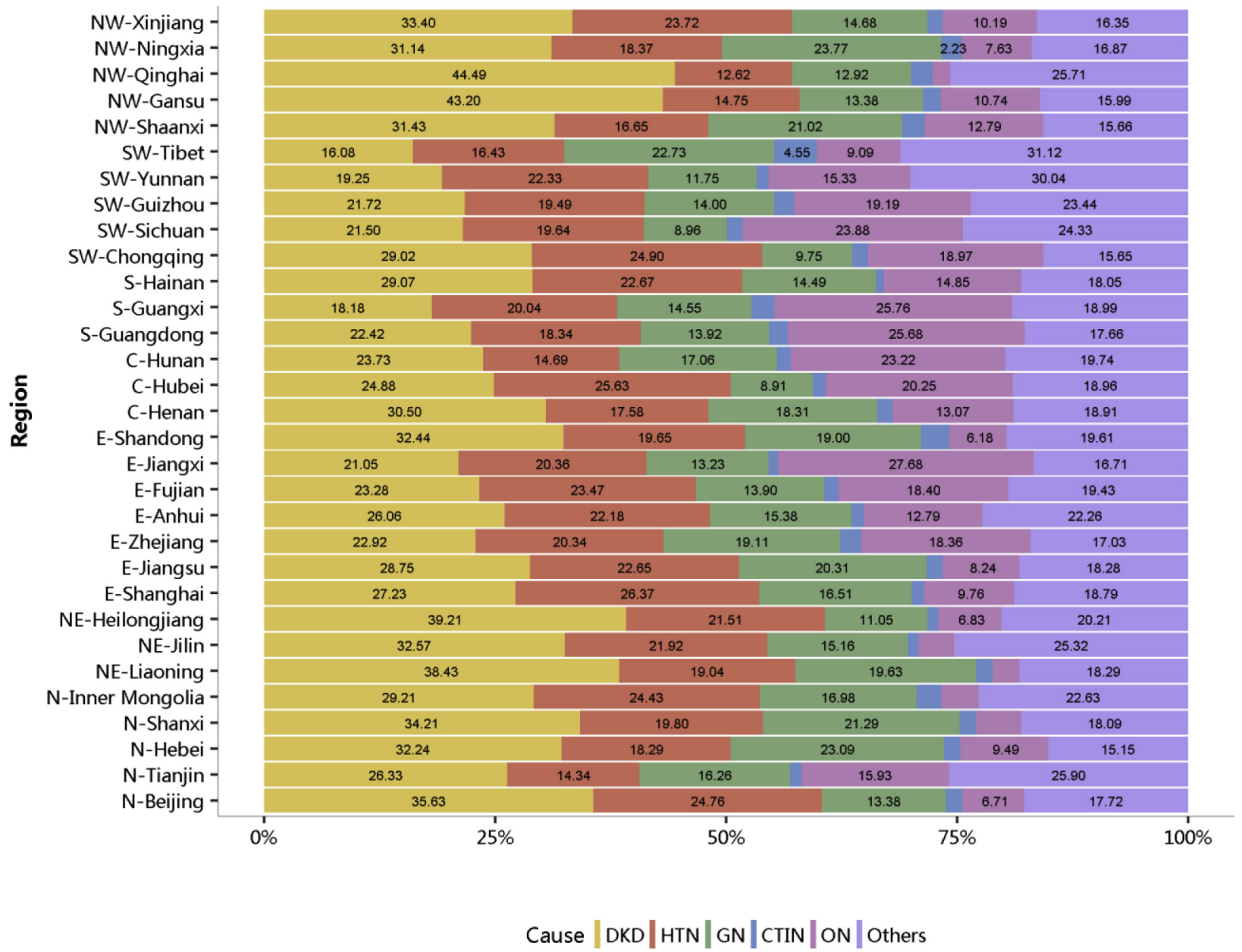

Figure 9 | Cause of CKD, stratified by geographic region. C, Central China; CKD, chronic kidney disease; CTIN, chronic tubulointerstitial nephritis; DKD, diabetic kidney disease; E, East China; GN, CKD due to glomerulonephritis; HTN, hypertensive nephropathy; N, North China; NE, Northeast China; NW, Northwest China; ON, obstructive nephropathy; Others, CKD due to other reasons; S, South China; SW, Southwest China. 
Table 8 | Cause of CKD, stratified by geographic region

\begin{tabular}{|c|c|c|c|c|c|c|c|}
\hline Region & DKD & HTN & GN & CTIN & ON & Others & Total \\
\hline N-Beijing & $9777(35.63)$ & $6794(24.76)$ & 3672 (13.38) & 490 (1.79) & $1842(6.71)$ & $4862(17.72)$ & 27,437 \\
\hline N-Tianjin & $1291(26.33)$ & 703 (14.34) & 797 (16.26) & $61(1.24)$ & $781(15.93)$ & $1270(25.90)$ & 4903 \\
\hline N-Hebei & $8114(32.24)$ & 4603 (18.29) & $5812(23.09)$ & $440(1.75)$ & $2389(9.49)$ & $3812(15.15)$ & 25,170 \\
\hline N-Shanxi & $6708(34.21)$ & 3882 (19.80) & $4174(21.29)$ & $353(1.80)$ & $943(4.81)$ & 3546 (18.09) & 19,606 \\
\hline N-Inner Mongolia & $5868(29.21)$ & $4908(24.43)$ & 3412 (16.98) & $544(2.71)$ & $811(4.04)$ & 4547 (22.63) & 20,090 \\
\hline NE-Liaoning & $10,275(38.43)$ & $5091(19.04)$ & $5250(19.63)$ & $468(1.75)$ & $765(2.86)$ & 4890 (18.29) & 26,739 \\
\hline NE-Jilin & $5569(32.57)$ & 3748 (21.92) & 2592 (15.16) & $202(1.18)$ & $658(3.85)$ & $4330(25.32)$ & 17,099 \\
\hline NE-Heilongjiang & $8200(39.21)$ & $4498(21.51)$ & $2312(11.05)$ & 249 (1.19) & $1429(6.83)$ & $4226(20.21)$ & 20,914 \\
\hline E-Shanghai & $6358(27.23)$ & $6158(26.37)$ & $3855(16.51)$ & $312(1.34)$ & $2280(9.76)$ & 4389 (18.79) & 23,352 \\
\hline E-Jiangsu & $14,406(28.75)$ & $11,346(22.65)$ & $10,175(20.31)$ & $885(1.77)$ & $4130(8.24)$ & 9159 (18.28) & 50,101 \\
\hline E-Zhejiang & $7938(22.92)$ & $7045(20.34)$ & $6618(19.11)$ & $781(2.25)$ & $6358(18.36)$ & $5897(17.03)$ & 34,637 \\
\hline E-Anhui & $7244(26.06)$ & $6165(22.18)$ & 4275 (15.38) & $374(1.35)$ & 3555 (12.79) & $6187(22.26)$ & 27,800 \\
\hline E-Fujian & $4980(23.28)$ & 5019 (23.47) & $2973(13.90)$ & $326(1.52)$ & 3936 (18.40) & 4155 (19.43) & 21,389 \\
\hline E-Jiangxi & 7990 (21.05) & 7731 (20.36) & $5023(13.23)$ & $367(0.97)$ & 10,509 (27.68) & $6344(16.71)$ & 37,964 \\
\hline E-Shandong & $10,422(32.44)$ & $6313(19.65)$ & 6105 (19.00) & $1003(3.12)$ & $1986(6.18)$ & 6302 (19.61) & 32,131 \\
\hline C-Henan & $11,785(30.50)$ & 6793 (17.58) & 7075 (18.31) & $629(1.63)$ & 5048 (13.07) & 7305 (18.91) & 38,635 \\
\hline C-Hubei & $18,360(24.88)$ & $18,918(25.63)$ & $6576(8.91)$ & 1007 (1.36) & $14,948(20.25)$ & $13,994(18.96)$ & 73,803 \\
\hline C-Hunan & $8454(23.73)$ & 5233 (14.69) & $6080(17.06)$ & $553(1.55)$ & $8275(23.22)$ & 7035 (19.74) & 35,630 \\
\hline S-Guangdong & $19,483(22.42)$ & 15,939 (18.34) & $12,102(13.92)$ & 1721 (1.98) & $22,315(25.68)$ & 15,353 (17.66) & 86,913 \\
\hline S-Guangxi & $5814(18.18)$ & $6411(20.04)$ & 4655 (14.55) & 795 (2.49) & $8238(25.76)$ & 6073 (18.99) & 31,986 \\
\hline S-Hainan & $2633(29.07)$ & 2053 (22.67) & 1312 (14.49) & $79(0.87)$ & 1345 (14.85) & 1635 (18.05) & 9057 \\
\hline SW-Chongqing & $3203(29.02)$ & 2749 (24.90) & 1076 (9.75) & $189(1.71)$ & 2094 (18.97) & $1728(15.65)$ & 11,039 \\
\hline SW-Sichuan & $12,247(21.50)$ & 11,188 (19.64) & $5104(8.96)$ & 963 (1.69) & $13,606(23.88)$ & $13,860(24.33)$ & 56,968 \\
\hline SW-Guizhou & $2779(21.72)$ & 2494 (19.49) & $1792(14.00)$ & $276(2.16)$ & 2455 (19.19) & $3000(23.44)$ & 12,796 \\
\hline SW-Yunnan & 8869 (19.25) & $10,286(22.33)$ & $5413(11.75)$ & $602(1.31)$ & 7061 (15.33) & $13,842(30.04)$ & 46,073 \\
\hline SW-Tibet & 46 (16.08) & 47 (16.43) & $65(22.73)$ & $13(4.55)$ & $26(9.09)$ & $89(31.12)$ & 286 \\
\hline NW-Shaanxi & 6565 (31.43) & 3477 (16.65) & $4391(21.02)$ & $513(2.46)$ & 2672 (12.79) & 3271 (15.66) & 20,889 \\
\hline NW-Gansu & $3512(43.20)$ & 1199 (14.75) & $1088(13.38)$ & 157 (1.93) & $873(10.74)$ & 1300 (15.99) & 8129 \\
\hline NW-Qinghai & 2365 (44.49) & $671(12.62)$ & $687(12.92)$ & $124(2.33)$ & $102(1.92)$ & $1367(25.71)$ & 5316 \\
\hline NW-Ningxia & 1119 (31.14) & 660 (18.37) & $854(23.77)$ & $80(2.23)$ & $274(7.63)$ & 606 (16.87) & 3593 \\
\hline NW-Xinjiang & $4838(33.40)$ & $3436(23.72)$ & $2127(14.68)$ & 239 (1.65) & 1476 (10.19) & 2369 (16.35) & 14,485 \\
\hline Total & $227,212(26.89)$ & $175,558(20.78)$ & $127,442(15.08)$ & 14,795 (1.75) & 133,180 (15.76) & 166,743 (19.73) & 844,930 \\
\hline
\end{tabular}

C, Central China; CKD, chronic kidney disease; CTIN, chronic tubulointerstitial nephritis; DKD, diabetic kidney disease; E, East China; GN, CKD due to glomerulonephritis; HTN, hypertensive nephropathy; N, North China; NE, Northeast China; NW, Northwest China; ON, obstructive nephropathy; Others, CKD due to other reasons; S, South China; SW, Southwest China.

Patients with missing data for geographic region were not included in the analysis; CKD: 42,837 (4.83\%). Patients of Macao, Hong Kong, and Taiwan were not included; CKD: 49 (0.01\%).

\subsection{Staging of CKD}

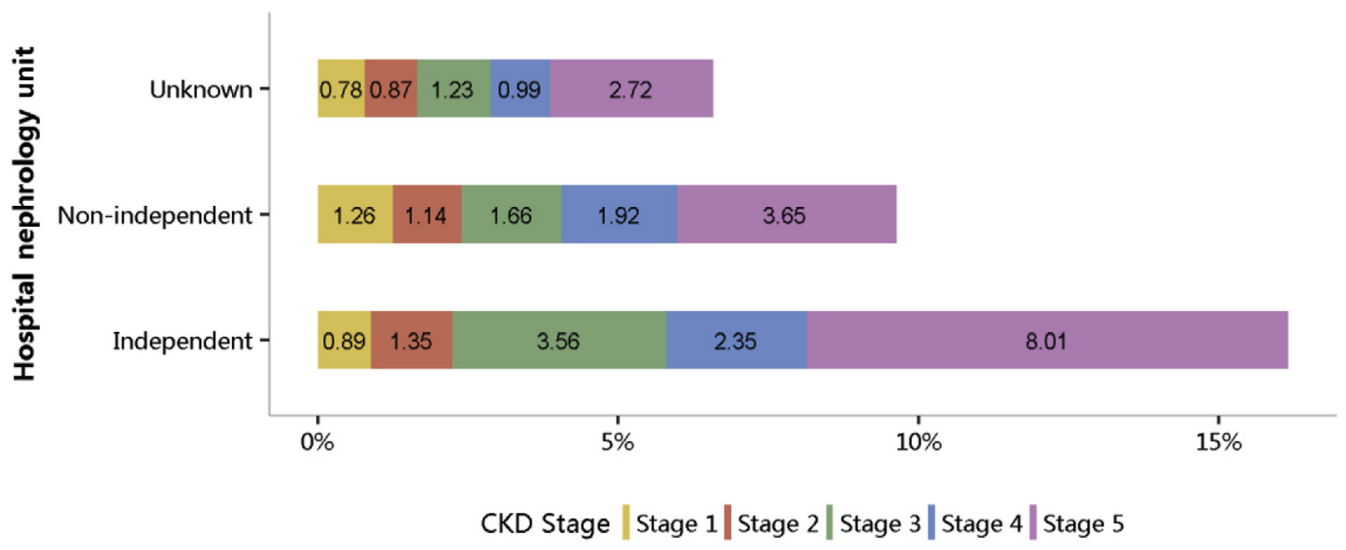

Figure 10| Staging of CKD. CKD, chronic kidney disease. 


\section{Table 9| Staging of CKD}

\begin{tabular}{|c|c|c|c|c|}
\hline CKD stage & Independent nephrology unit & Non-independent nephrology unit & Unknown & Total \\
\hline Stage 1 & $6997(0.89)$ & $530(1.26)$ & $477(0.78)$ & $8,004(0.90)$ \\
\hline Stage 2 & $10,585(1.35)$ & $482(1.14)$ & $535(0.87)$ & $11,602(1.31)$ \\
\hline Stage 3 & $27,893(3.56)$ & $699(1.66)$ & $754(1.23)$ & $29,346(3.31)$ \\
\hline Stage 5 & $62,798(8.01)$ & 1539 (3.65) & 1665 (2.72) & $66,002(7.43)$ \\
\hline Unknown & $657,631(83.85)$ & $38,081(90.36)$ & $57,254(93.41)$ & $752,966(84.82)$ \\
\hline Total & 784,330 & 42,142 & 61,291 & 887,763 \\
\hline
\end{tabular}

CKD, chronic kidney disease.

Patients with missing and/or controversial data for stage were not included in the analysis; CKD: 53 (0.01\%). 


\section{Chapter 2: Cardiovascular disease in hospitalized patients with CKD}

Bixia Gao ${ }^{1}$, Jianyan Long ${ }^{2}$, Kunhao $\mathrm{Bai}^{3}$, Lanxia $\mathrm{Gan}^{4}$, Zaiming $\mathrm{Su}^{5}$, Fang Wang ${ }^{1}$ and Haibo Wang ${ }^{4,5}$

${ }^{1}$ Renal Division, Department of Medicine, Peking University First Hospital; Peking University Institute of Nephrology, Beijing, China; ${ }^{2}$ Clinical Trial Unit, The First Affiliated Hospital of Sun Yat-sen University, Guangzhou, China; ${ }^{3}$ Department of Endoscopy, Sun Yatsen University Cancer Center, State Key Laboratory of Oncology in South China, Collaborative Innovation Center for Cancer Medicine, Guangzhou, China; ${ }^{4}$ China Standard Medical Information Research Center, Shenzhen, China; and ${ }^{5}$ Center for Data Science in Health and Medicine, Peking University, Beijing, China

This chapter focuses on the clinical pattern and treatment of CVD in hospitalized patients with CKD.

Regarding the clinical pattern of CVD among inpatients with $\mathrm{CKD}$, coronary heart disease (CHD) was most common $(18.22 \%)$, followed by heart failure $(16.02 \%)$, stroke (12.88\%), and atrial fibrillation (3.74\%) (Figure 11 and Table 10). The percentages of those CVDs among patients with CKD were all higher compared with patients without CKD. Results of patients with diabetes were also included for comparison. Patients with CKD had lower percentages for CHD and stroke (18.22\% vs. $27.62 \%, 12.88 \%$ vs. $21.67 \%)$, and higher percentages for heart failure and atrial fibrillation ( $16.02 \%$ vs. $12.93 \%, 3.74 \%$ vs. $3.53 \%)$, compared with patients with diabetes mellitus (DM). The trends were consistent across various age and sex groups (Figures 12-19 and Tables 11-18).
Among the causes of CKD, patients with diabetic kidney disease (DKD), hypertensive nephropathy (HTN), and chronic tubule-interstitial nephritis (CTIN) had relatively high percentage of CVD (Figures 20-28 and Tables 19-27).

Despite the relatively high burden of CHD among patients with CKD compared with those without CKD, the percentages of related procedures including coronarography (CAG), percutaneous coronary intervention (PCI), and coronary artery bypass graft (CABG) were much lower (Figure 29 and Table 28). The trend did not vary substantially across causes of CKD, except for patients with obstructive nephropathy (ON) (with lowest percentage of CHD and highest percentage of CAG and PCI) (Figures 30-35 and Tables 29-34). The percentage of pacemaker implantation among patients with CKD was higher compared with patients without CKD or patients with DM (Figures 36-38 and Tables 35-37).

\subsection{Prevalence of CVD, stratified by patient group}

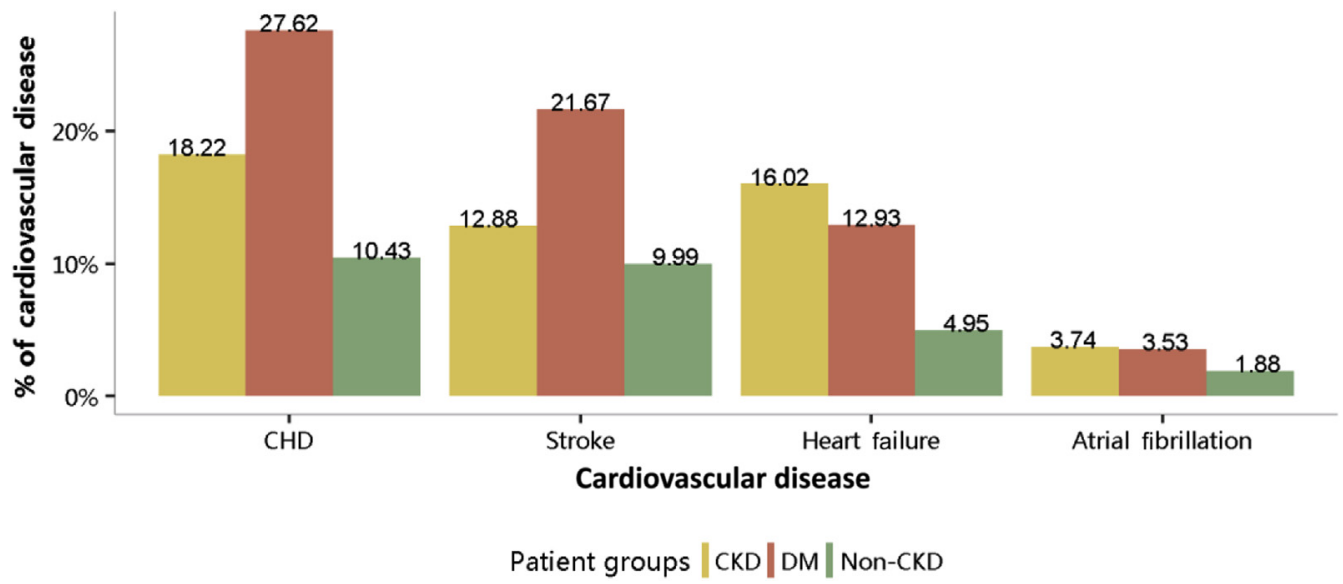

Figure 11 | Prevalence of CVD, stratified by patient group. CHD, coronary heart disease; CKD, chronic kidney disease; CVD, cardiovascular disease; DM, diabetes mellitus.

Table 10| Prevalence of CVD, stratified by patient group, $N(\%)$

\begin{tabular}{lrrrr}
\hline Patient group & CHD & Stroke & Heart failure & Atrial fibrillation \\
\hline CKD & $161,736(18.22)$ & $114,348(12.88)$ & $142,208(16.02)$ & $33,211(3.74)$ \\
DM & $481,419(27.62)$ & $377,695(21.67)$ & $225,458(12.93)$ & $61,472(3.53)$ \\
Non-CKD & $1,835,401(10.43)$ & $1,758,388(9.99)$ & $870,663(4.95)$ & $330,837(1.88)$ \\
\hline
\end{tabular}

CHD, coronary heart disease; CKD, chronic kidney disease; CVD, cardiovascular disease; DM, diabetes mellitus. 


\subsubsection{Prevalence of CHD.}

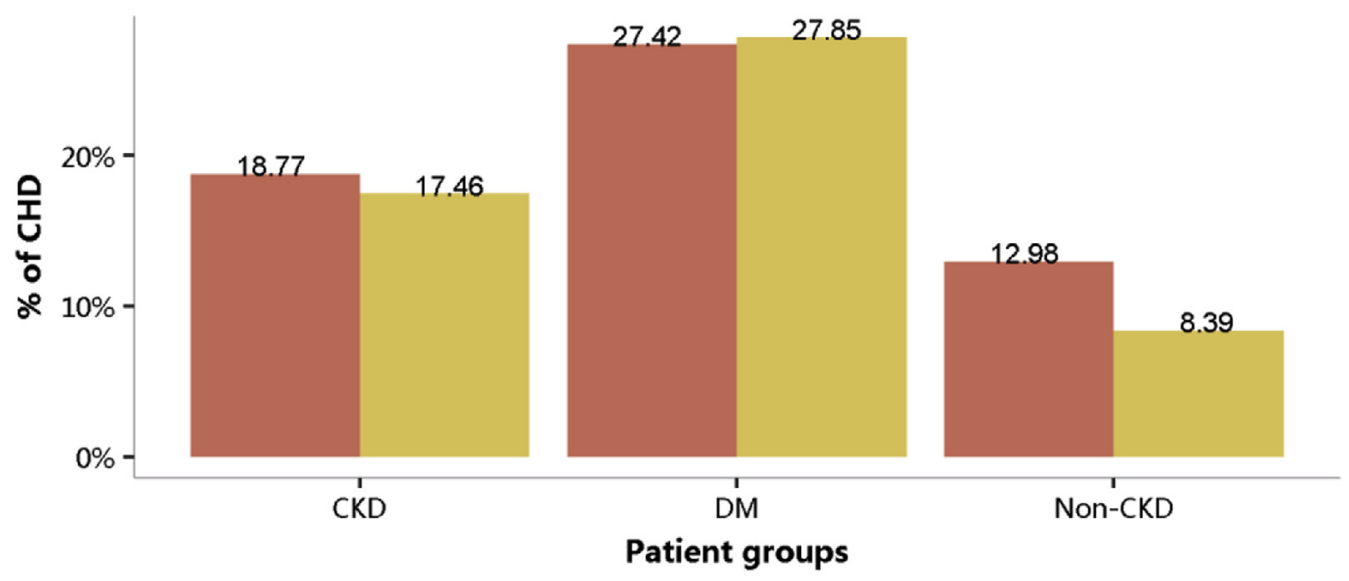

$$
\text { Sex |Male | Female }
$$

Figure 12 | Prevalence of CHD, stratified by sex. CHD, coronary heart disease; CKD, chronic kidney disease; DM, diabetes mellitus.

Table 11 | Prevalence of CHD, stratified by sex, $N(\%)$

\begin{tabular}{lccc}
\hline Sex & CKD & DM & Non-CKD \\
\hline Male & $96,511(18.77)$ & $258,358(27.42)$ & $1,012,693(12.98)$ \\
Female & $65,225(17.46)$ & $223,061(27.85)$ & $822,708(8.39)$ \\
Total & $161,736(18.22)$ & $481,419(27.62)$ & $1,835,401(10.43)$ \\
\hline
\end{tabular}

CHD, coronary heart disease; CKD, chronic kidney disease; DM, diabetes mellitus.

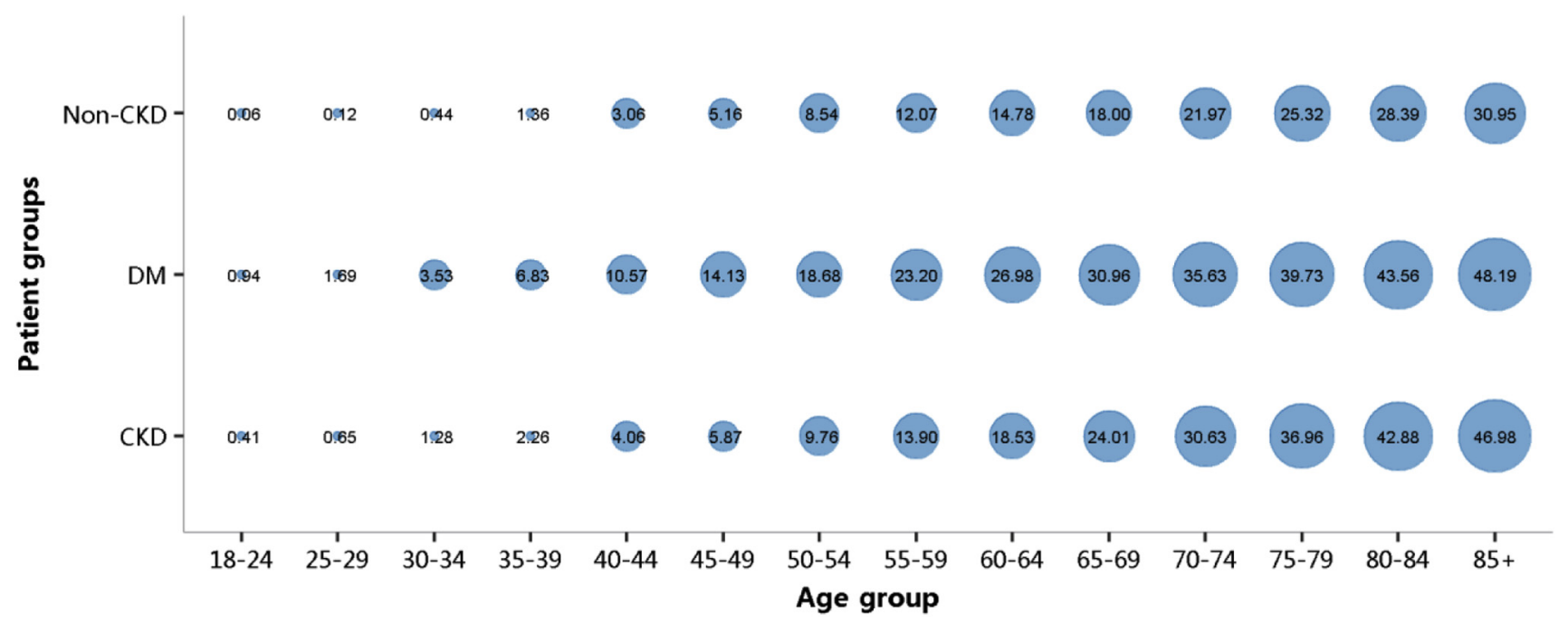

Figure 13 | Prevalence of CHD, stratified by age. CHD, coronary heart disease; CKD, chronic kidney disease; DM, diabetes mellitus. Point size refers to the percentage of CHD. 
Table 12 | Prevalence of CHD, stratified by age, $N$ (\%)

\begin{tabular}{lrrr}
\hline Age group & \multicolumn{1}{c}{ CKD } & \multicolumn{1}{c}{ DM } & Non-CKD \\
\hline $18-24$ & $100(0.41)$ & $63(0.94)$ & $552(0.06)$ \\
$25-29$ & $220(0.65)$ & $204(1.69)$ & $1,842(0.12)$ \\
$30-34$ & $455(1.28)$ & $689(3.53)$ & $5341(0.44)$ \\
$35-39$ & $932(2.26)$ & $2246(6.83)$ & $14,394(1.36)$ \\
$40-44$ & $2465(4.06)$ & $7279(10.57)$ & $40,062(3.06)$ \\
$45-49$ & $4632(5.87)$ & $16,796(14.13)$ & $81,007(5.16)$ \\
$50-54$ & $8936(9.76)$ & $36,248(18.68)$ & $145,936(8.54)$ \\
$55-59$ & $12,088(13.90)$ & $51,359(23.20)$ & $198,582(12.07)$ \\
$60-64$ & $18,925(18.53)$ & $74,592(26.98)$ & $276,335(14.78)$ \\
$65-69$ & $21,747(24.01)$ & $78,341(30.96)$ & $274,486(18.00)$ \\
$70-74$ & $23,818(30.63)$ & $75,440(35.63)$ & $265,626(21.97)$ \\
$75-79$ & $27,921(36.96)$ & $69,403(39.73)$ & $254,132(25.32)$ \\
$80-84$ & $23,468(42.88)$ & $46,086(43.56)$ & $178,726(28.39)$ \\
$85+$ & $16,029(46.98)$ & $22,673(48.19)$ & $98,380(30.95)$ \\
Total & $161,736(18.22)$ & $481,419(27.62)$ & $1,835,401(10.43)$ \\
\hline
\end{tabular}

$\mathrm{CHD}$, coronary heart disease; CKD, chronic kidney disease; DM, diabetes mellitus.

\subsubsection{Prevalence of stroke.}

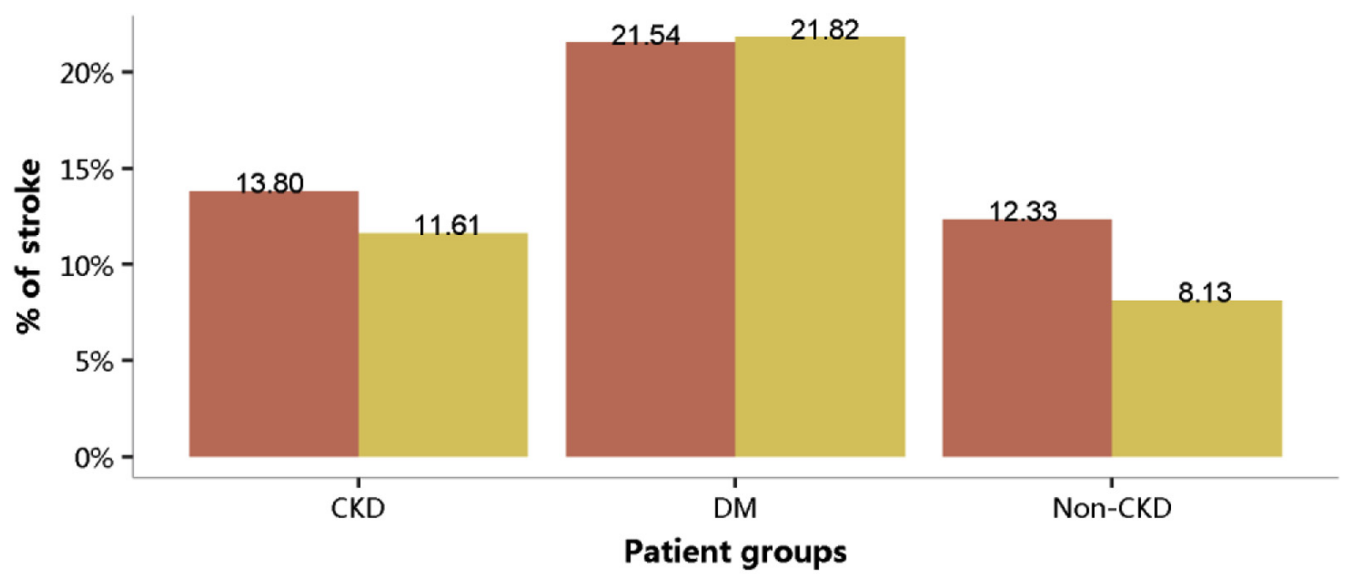

$$
\text { Sex | Male | Female }
$$

Figure 14| Prevalence of stroke, stratified by sex. CKD, chronic kidney disease; DM, diabetes mellitus.

Table 13 | Prevalence of stroke, stratified by sex, $N$ (\%)

\begin{tabular}{lrrr}
\hline Sex & CKD & DM & Non-CKD \\
\hline Male & $70,992(13.80)$ & $202,926(21.54)$ & $961,447(12.33)$ \\
Female & $43,356(11.61)$ & $174,769(21.82)$ & $796,941(8.13)$ \\
Total & $114,348(12.88)$ & $377,695(21.67)$ & $1,758,388(9.99)$ \\
\hline
\end{tabular}

CKD, chronic kidney disease; DM, diabetes mellitus. 


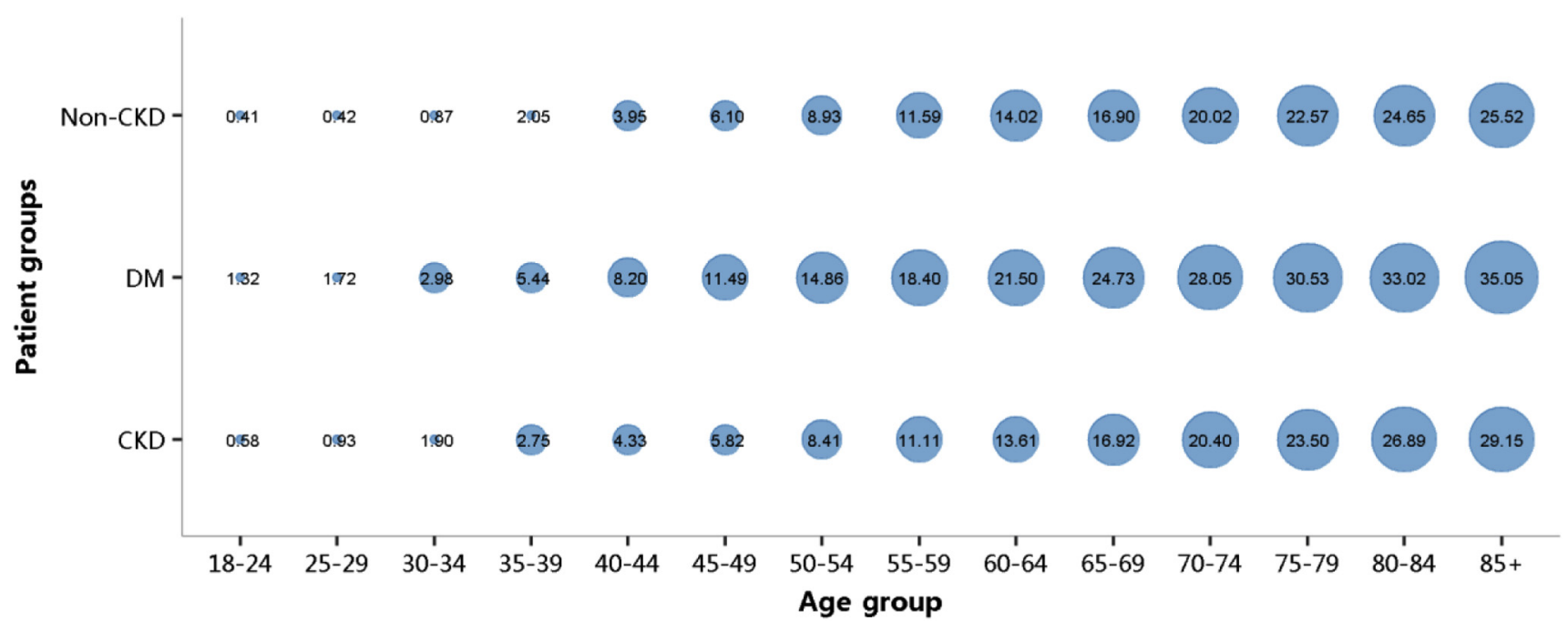

Figure 15 | Prevalence of stroke, stratified by age. CKD, chronic kidney disease; DM, diabetes mellitus. Point size refers to the percentage of stroke.

Table 14 | Prevalence of stroke, stratified by age, $N$ (\%)

\begin{tabular}{lrrr}
\hline Age group & \multicolumn{1}{c}{ CKD } & \multicolumn{1}{c}{ DM } & Non-CKD \\
\hline $18-24$ & $141(0.58)$ & $88(1.32)$ & $3992(0.41)$ \\
$25-29$ & $314(0.93)$ & $207(1.72)$ & $6645(0.42)$ \\
$30-34$ & $673(1.90)$ & $582(2.98)$ & $10,558(0.87)$ \\
$35-39$ & $1136(2.75)$ & $1789(5.44)$ & $21,628(2.05)$ \\
$40-44$ & $2629(4.33)$ & $5,644(8.20)$ & $51,794(3.95)$ \\
$45-49$ & $4593(5.82)$ & $13,663(11.49)$ & $95,828(6.10)$ \\
$50-54$ & $7703(8.41)$ & $28,833(14.86)$ & $152,576(8.93)$ \\
$55-59$ & $9664(11.11)$ & $40,727(18.40)$ & $190,800(11.59)$ \\
$60-64$ & $13,900(13.61)$ & $59,437(21.50)$ & $262,046(14.02)$ \\
$65-69$ & $15,324(16.92)$ & $62,572(24.73)$ & $257,663(16.90)$ \\
$70-74$ & $15,861(20.40)$ & $59,403(28.05)$ & $242,042(20.02)$ \\
$75-79$ & $17,750(23.50)$ & $53,323(30.53)$ & $226,539(22.57)$ \\
$80-84$ & $14,714(26.89)$ & $34,936(33.02)$ & $155,137(24.65)$ \\
$85+$ & $9946(29.15)$ & $16,491(35.05)$ & $81,140(25.52)$ \\
Total & $114,348(12.88)$ & $377,695(21.67)$ & $1,758,388(9.99)$ \\
\hline
\end{tabular}

CKD, chronic kidney disease; DM, diabetes mellitus.

\subsubsection{Prevalence of heart failure.}

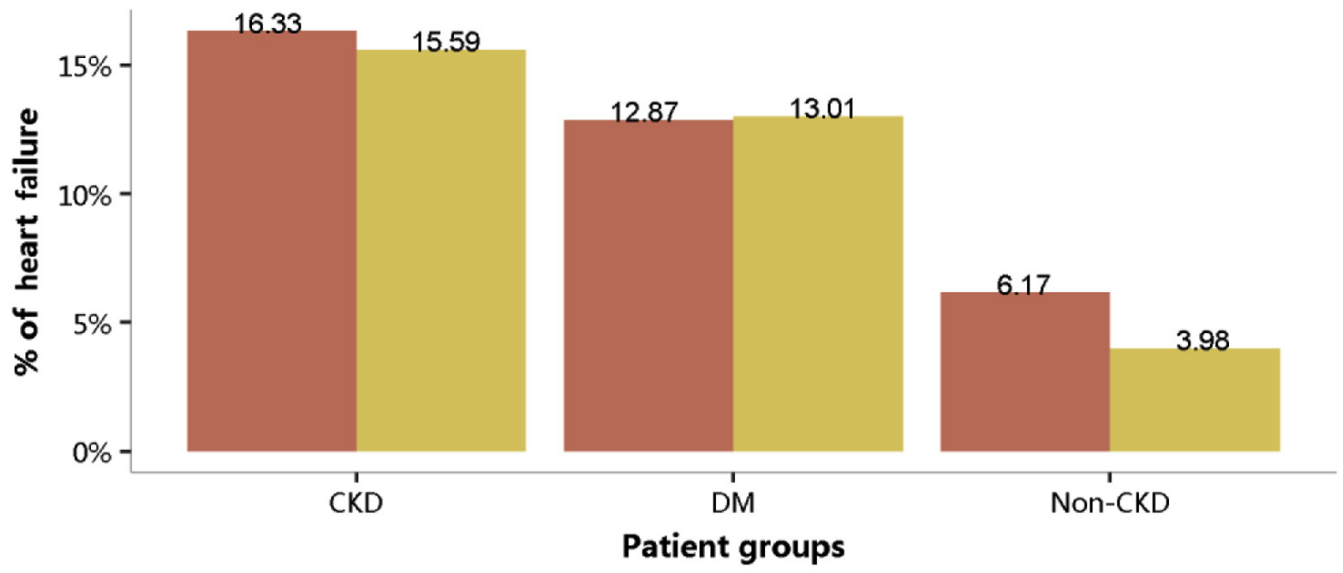

$$
\text { Sex | Male | Female }
$$

Figure 16| Prevalence of heart failure, stratified by sex. CKD, chronic kidney disease; DM, diabetes mellitus. 
Table 15 | Prevalence of heart failure, stratified by sex, $N$ (\%)

\begin{tabular}{lrrr}
\hline Sex & CKD & DM & Non-CKD \\
\hline Male & $83,980(16.33)$ & $121,233(12.87)$ & $480,970(6.17)$ \\
Female & $58,228(15.59)$ & $104,225(13.01)$ & $389,693(3.98)$ \\
Total & $142,208(16.02)$ & $225,458(12.93)$ & $870,663(4.95)$ \\
\hline
\end{tabular}

CKD, chronic kidney disease; DM, diabetes mellitus.

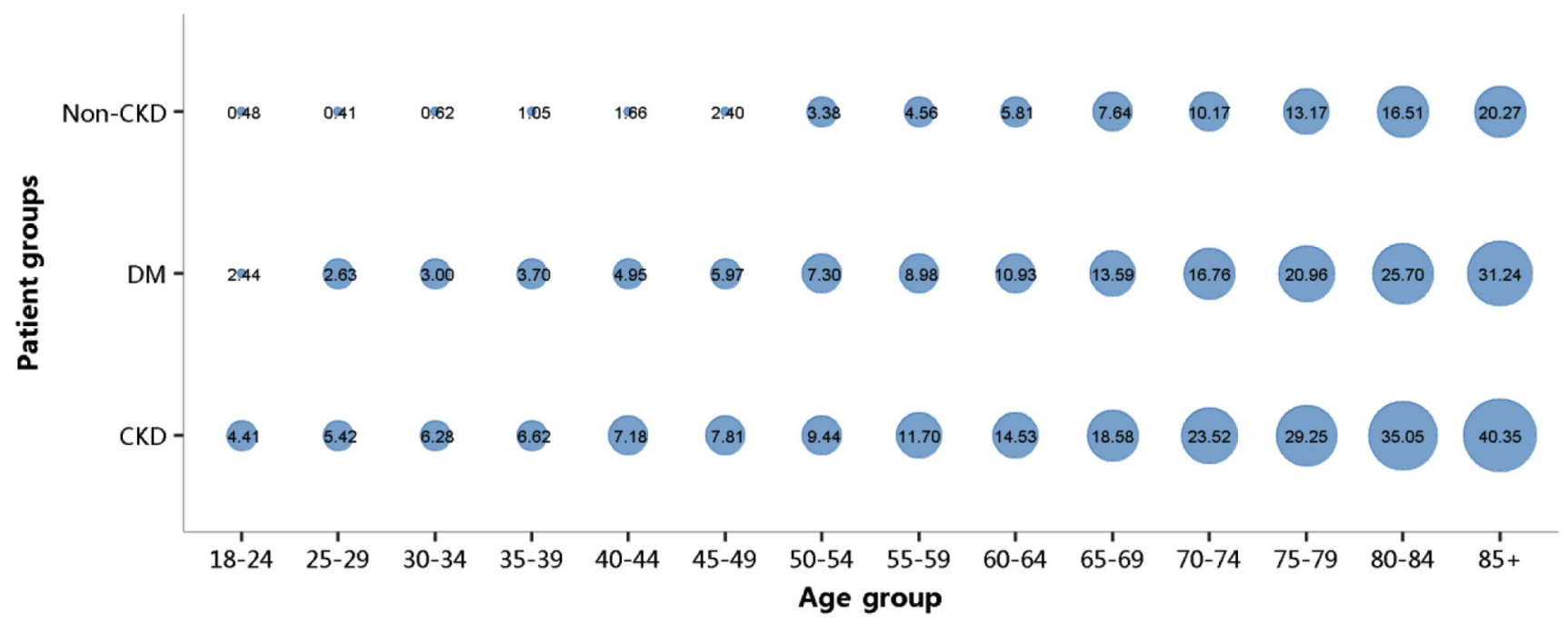

Figure 17| Prevalence of heart failure, stratified by age. CKD, chronic kidney disease; DM, diabetes mellitus. Point size refers to the percentage of heart failure.

Table 16| Prevalence of heart failure, stratified by age, $N(\%)$

\begin{tabular}{lcrr}
\hline Age group & CKD & \multicolumn{1}{c}{ DM } & \multicolumn{1}{c}{ Non-CKD } \\
\hline $18-24$ & $1073(4.41)$ & $163(2.44)$ & $4627(0.48)$ \\
$25-29$ & $1826(5.42)$ & $317(2.63)$ & $6394(0.41)$ \\
$30-34$ & $2230(6.28)$ & $586(3.00)$ & $7576(0.62)$ \\
$35-39$ & $2729(6.62)$ & $1217(3.70)$ & $11,089(1.05)$ \\
$40-44$ & $4354(7.18)$ & $3407(4.95)$ & $21,749(1.66)$ \\
$45-49$ & $6165(7.81)$ & $7091(5.97)$ & $37,671(2.40)$ \\
$50-54$ & $8650(9.44)$ & $14,178(7.30)$ & $57,745(3.38)$ \\
$55-59$ & $10,178(11.70)$ & $19,877(8.98)$ & $75,069(4.56)$ \\
$60-64$ & $14,836(14.53)$ & $30,233(10.93)$ & $108,605(5.81)$ \\
$65-69$ & $16,835(18.58)$ & $35,392(13.59)$ & $16,598(16.76)$ \\
$70-74$ & $18,288(23.52)$ & $36,618(20.96)$ & $122,991(10.17)$ \\
$75-79$ & $22,098(29.25)$ & $27,184(25.70)$ & $132,235(13.17)$ \\
$80-84$ & $19,179(35.05)$ & $14,697(31.24)$ & $103,940(16.51)$ \\
$85+$ & $13,767(40.35)$ & $225,458(12.93)$ & $64,426(20.27)$ \\
Total & $142,208(16.02)$ & & $870,663(4.95)$ \\
\hline
\end{tabular}

CKD, chronic kidney disease; DM, diabetes mellitus. 


\subsubsection{Prevalence of atrial fibrillation.}

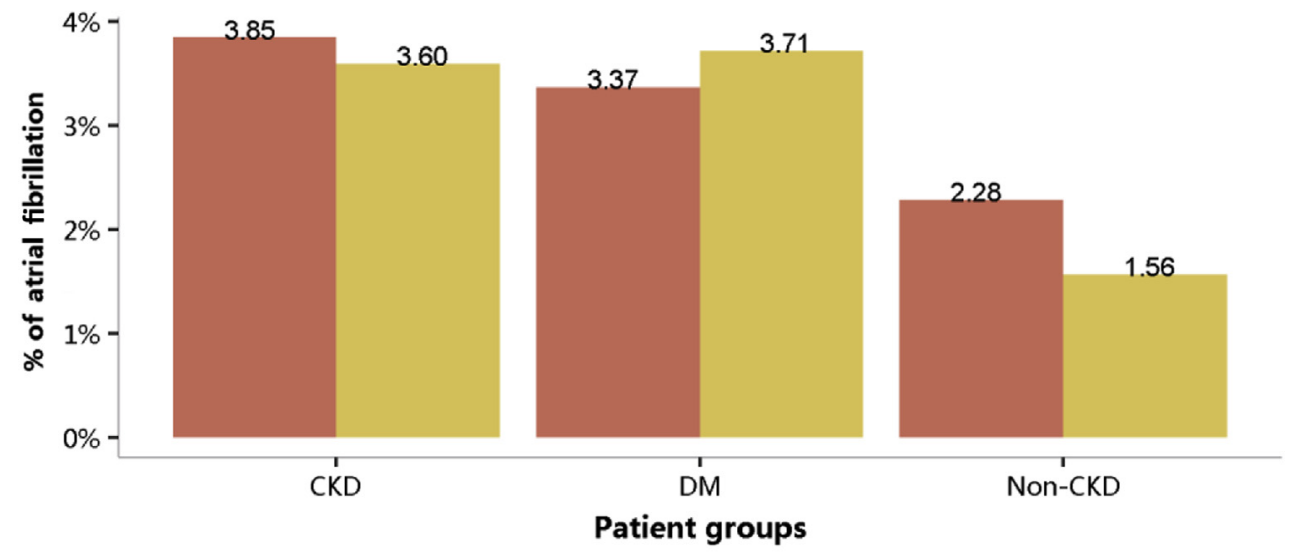

Sex |Male | Female

Figure 18| Prevalence of atrial fibrillation, stratified by sex. CKD, chronic kidney disease; DM, diabetes mellitus.

Table 17| Prevalence of atrial fibrillation, stratified by sex, $N(\%)$

\begin{tabular}{lccr}
\hline Sex & CKD & DM & Non-CKD \\
\hline Male & $19,782(3.85)$ & $31,728(3.37)$ & $177,730(2.28)$ \\
Female & $13,429(3.60)$ & $29,744(3.71)$ & $153,107(1.56)$ \\
Total & $33,211(3.74)$ & $61,472(3.53)$ & $330,837(1.88)$ \\
\hline
\end{tabular}

CKD, chronic kidney disease; DM, diabetes mellitus.

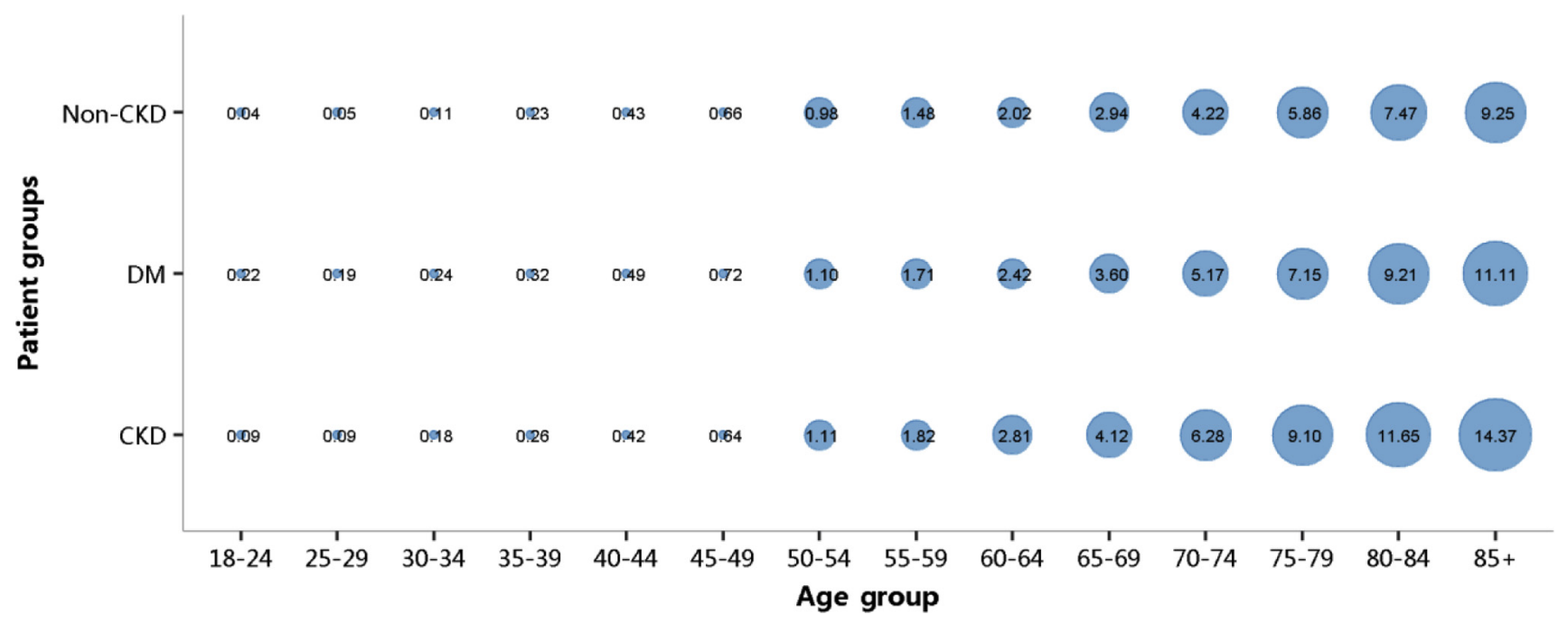

Figure 19| Prevalence of atrial fibrillation, stratified by age. CKD, chronic kidney disease; DM, diabetes mellitus. Point size refers to the percentage of atrial fibrillation. 
Table 18 | Prevalence of atrial fibrillation, stratified by age, $N$ (\%)

\begin{tabular}{lcrr}
\hline Age group & \multicolumn{1}{c}{ CKD } & \multicolumn{1}{c}{ DM } & Non-CKD \\
\hline $18-24$ & $22(0.09)$ & $15(0.22)$ & $427(0.04)$ \\
$25-29$ & $30(0.09)$ & $23(0.19)$ & $780(0.05)$ \\
$30-34$ & $63(0.18)$ & $46(0.24)$ & $1334(0.11)$ \\
$35-39$ & $108(0.26)$ & $105(0.32)$ & $2442(0.23)$ \\
$40-44$ & $256(0.42)$ & $337(0.49)$ & $5581(0.43)$ \\
$45-49$ & $502(0.64)$ & $851(0.72)$ & $10,400(0.66)$ \\
$50-54$ & $1016(1.11)$ & $2126(1.10)$ & $16,761(0.98)$ \\
$55-59$ & $1579(1.82)$ & $3775(1.71)$ & $24,310(1.48)$ \\
$60-64$ & $2868(2.81)$ & $6678(2.42)$ & $37,802(2.02)$ \\
$65-69$ & $3733(4.12)$ & $9122(3.60)$ & $44,785(2.94)$ \\
$70-74$ & $4882(6.28)$ & $12,940(5.17)$ & $51,004(4.22)$ \\
$75-79$ & $6873(9.10)$ & $9740(9.15)$ & $58,787(5.86)$ \\
$80-84$ & $6376(11.65)$ & $5227(11.11)$ & $47,029(7.47)$ \\
$85+$ & $4903(14.37)$ & $61,472(3.53)$ & $29,395(9.25)$ \\
Total & $33,211(3.74)$ & & $330,837(1.88)$ \\
\hline
\end{tabular}

CKD, chronic kidney disease; DM, diabetes mellitus.

\subsection{Prevalence of CVD among patients with CKD}

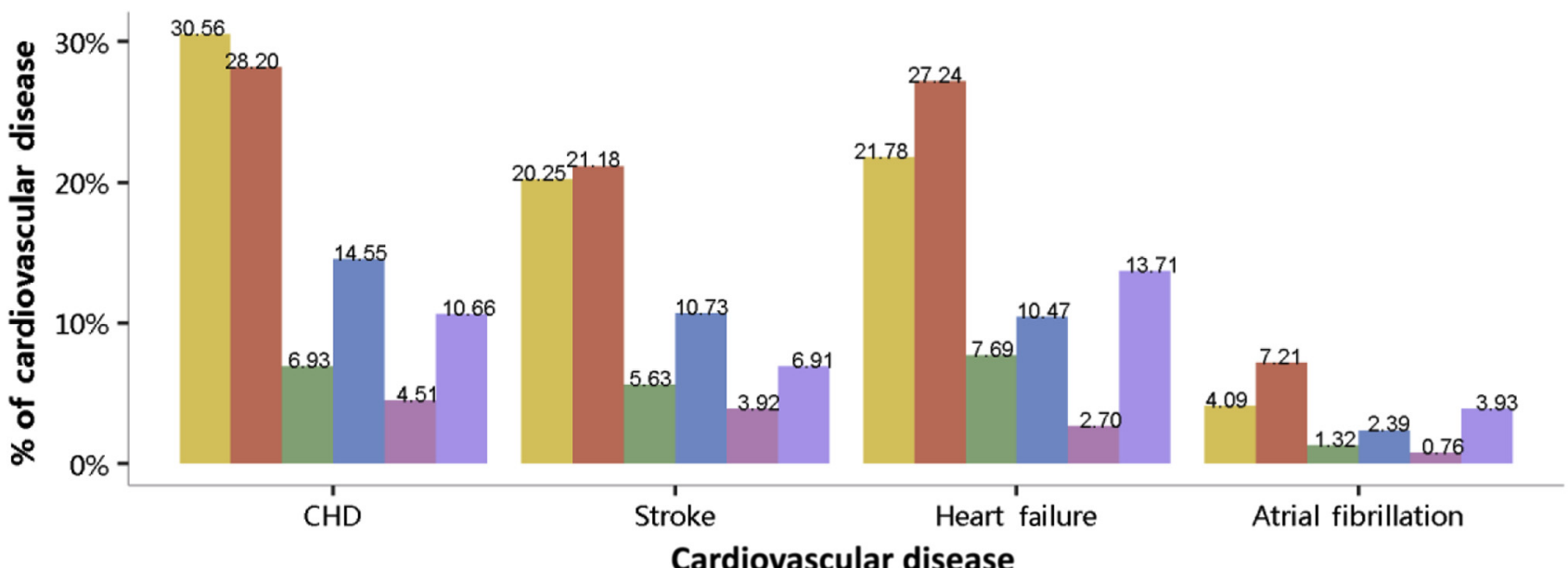

\section{Cause |DKD |HTN |GN|CTIN |ON |Others}

Figure 20| Prevalence of CVD among patients with CKD. CHD, coronary heart disease; CKD, chronic kidney disease; CTIN, chronic tubulointerstitial nephritis; CVD, cardiovascular disease; DKD, diabetic kidney disease; GN, CKD due to glomerulonephritis; HTN, hypertensive nephropathy; ON, obstructive nephropathy; Others, CKD due to other reasons.

Table 19| Prevalence of CVD among patients with CKD, $N$ (\%)

\begin{tabular}{lccrr}
\hline Cause & CHD & Stroke & Heart failure & Atrial fibrillation \\
\hline DKD & $73,148(30.56)$ & $48,469(20.25)$ & $52,138(21.78)$ & $9794(4.09)$ \\
HTN & $52,031(28.20)$ & $39,072(21.18)$ & $50,252(27.24)$ & $13,305(7.21)$ \\
GN & $9269(6.93)$ & $7537(5.63)$ & $10,295(7.69)$ & $1764(1.32)$ \\
CTIN & $2258(14.55)$ & $1666(10.73)$ & $1626(10.47)$ & $371(2.39)$ \\
ON & $6243(4.51)$ & $5420(3.92)$ & $3731(2.70)$ & $1055(0.76)$ \\
Others & $18,787(10.66)$ & $12,184(6.91)$ & $24,166(13.71)$ & $6922(3.93)$ \\
Total & $161,736(18.22)$ & $114,348(12.88)$ & $142,208(16.02)$ & $33,211(3.74)$ \\
\hline
\end{tabular}

CHD, coronary heart disease; CKD, chronic kidney disease; CTIN, chronic tubulointerstitial nephritis; CVD, cardiovascular disease; DKD, diabetic kidney disease; GN, CKD due to glomerulonephritis; HTN, hypertensive nephropathy; ON, obstructive nephropathy; Others, CKD due to other reasons. 


\subsubsection{Prevalence of CHD among patients with CKD.}

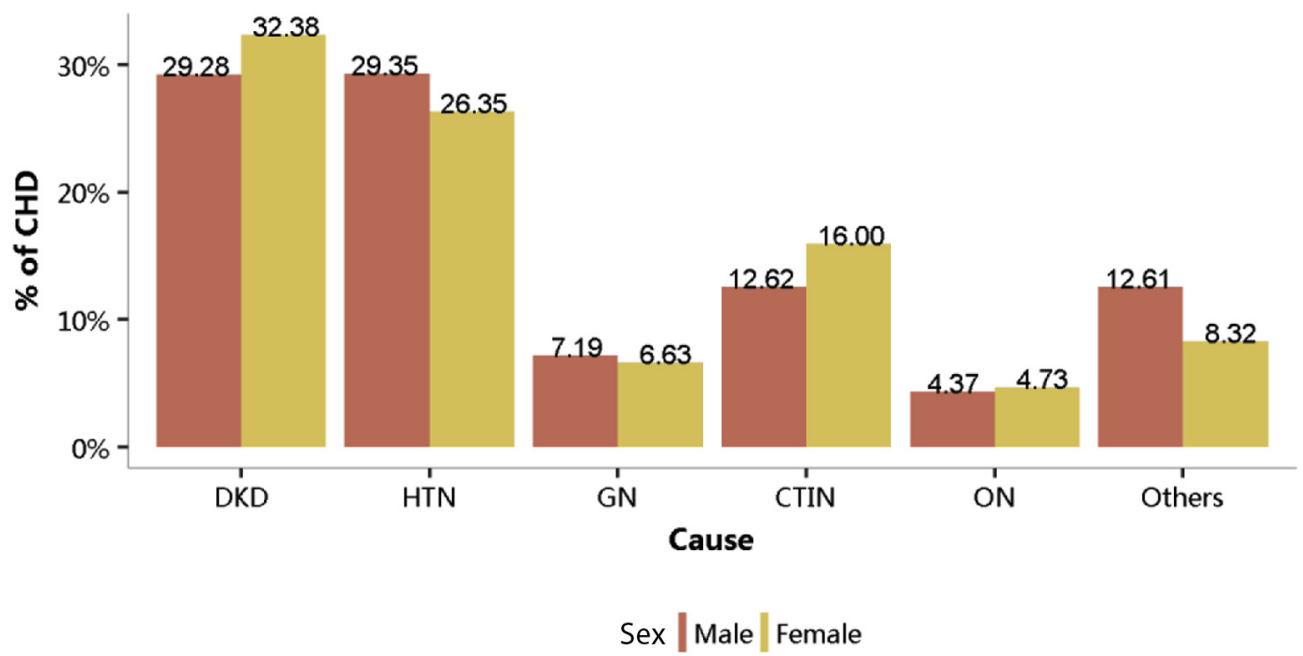

Figure 21 | Prevalence of CHD among patients with CKD, stratified by cause and sex. CHD, coronary heart disease; CKD, chronic kidney disease; CTIN, chronic tubulointerstitial nephritis; DKD, diabetic kidney disease; GN, CKD due to glomerulonephritis; HTN, hypertensive nephropathy; ON, obstructive nephropathy; Others, CKD due to other reasons.

Table 20| Prevalence of CHD among patients with CKD, stratified by cause and sex, $N$ (\%)

\begin{tabular}{lcccrrrr}
\hline Sex & DKD & HTN & GN & CTIN & ON & Others \\
\hline Male & $41,176(29.28)$ & $33,500(29.35)$ & $5148(7.19)$ & $846(12.62)$ & $3720(4.37)$ & $12,121(12.61)$ & $96,511(18.77)$ \\
Female & $31,972(32.38)$ & $18,531(26.35)$ & $4121(6.63)$ & $1412(16.00)$ & $2523(4.73)$ & $6666(8.32)$ & $65,225(17.46)$ \\
Total & $73,148(30.56)$ & $52,031(28.20)$ & $9269(6.93)$ & $2258(14.55)$ & $6243(4.51)$ & $18,787(10.66)$ & $161,736(18.22)$ \\
\hline
\end{tabular}

CHD, coronary heart disease; CKD, chronic kidney disease; CTIN, chronic tubulointerstitial nephritis; DKD, diabetic kidney disease; GN, CKD due to glomerulonephritis; HTN, hypertensive nephropathy; ON, obstructive nephropathy; Others, CKD due to other reasons.

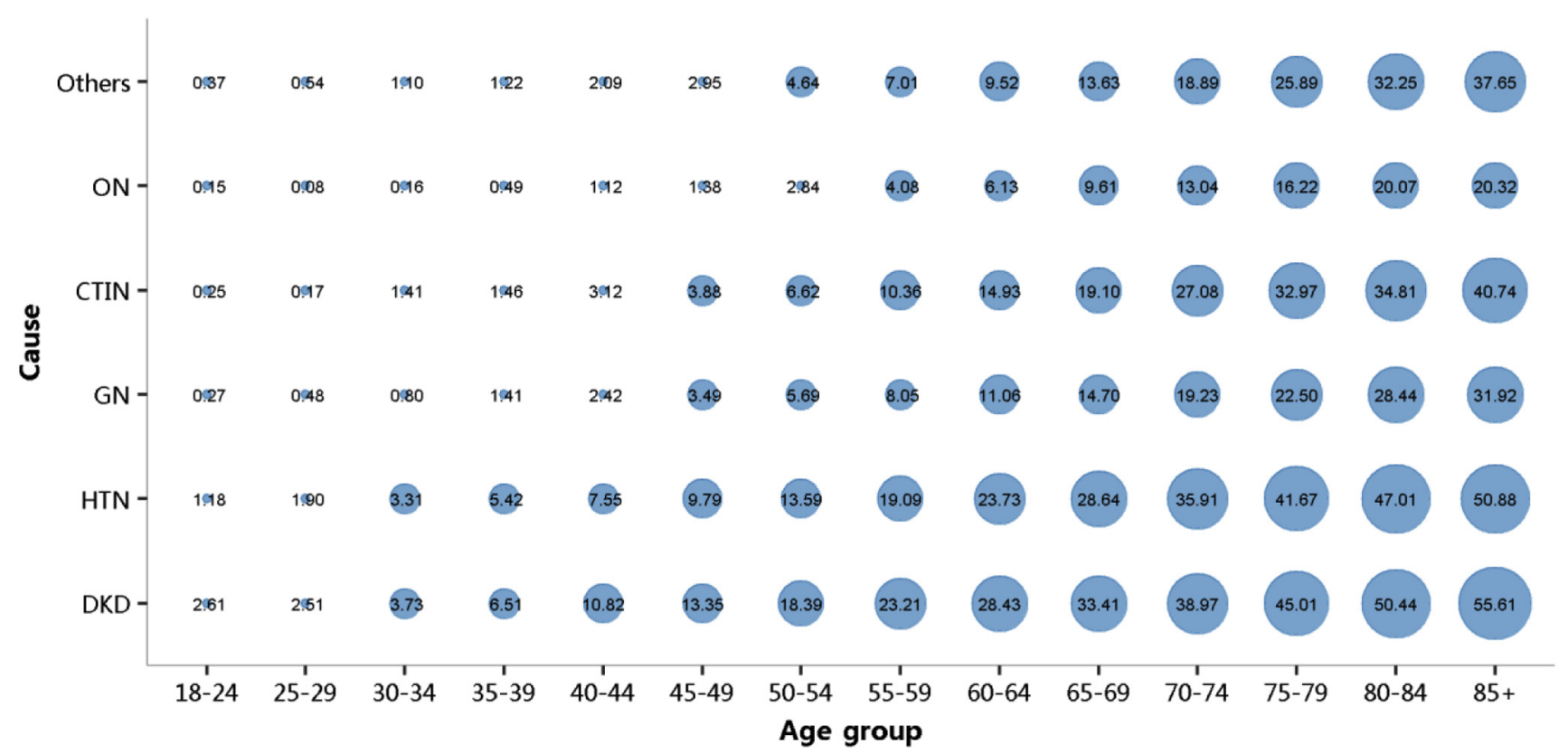

Figure 22 | Prevalence of CHD among patients with CKD, stratified by cause and sex. CHD, coronary heart disease; CKD, chronic kidney disease; CTIN, chronic tubulointerstitial nephritis; DKD, diabetic kidney disease; GN, CKD due to glomerulonephritis; HTN, hypertensive nephropathy; ON, obstructive nephropathy; Others, CKD due to other reasons. Point size refers to the percentage of CHD. 
Table 21 | Prevalence of CHD among patients with CKD, stratified by cause and age, $N$ (\%)

\begin{tabular}{|c|c|c|c|c|c|c|c|}
\hline Age group & DKD & HTN & GN & CTIN & ON & Others & Total \\
\hline $18-24$ & $19(2.61)$ & $18(1.18)$ & $27(0.27)$ & $1(0.25)$ & $6(0.15)$ & $29(0.37)$ & $100(0.41)$ \\
\hline $25-29$ & $41(2.51)$ & $62(1.90)$ & $54(0.48)$ & $1(0.17)$ & $5(0.08)$ & $57(0.54)$ & $220(0.65)$ \\
\hline $30-34$ & $99(3.73)$ & $149(3.31)$ & $81(0.80)$ & $8(1.41)$ & $13(0.16)$ & $105(1.10)$ & 455 (1.28) \\
\hline $35-39$ & $283(6.51)$ & $319(5.42)$ & $147(1.41)$ & $10(1.46)$ & $47(0.49)$ & $126(1.22)$ & $932(2.26)$ \\
\hline $40-44$ & 962 (10.82) & $694(7.55)$ & $329(2.42)$ & $32(3.12)$ & $156(1.12)$ & 292 (2.09) & 2465 (4.06) \\
\hline $45-49$ & 2137 (13.35) & 1181 (9.79) & $523(3.49)$ & $54(3.88)$ & $246(1.38)$ & 491 (2.95) & $4632(5.87)$ \\
\hline $50-54$ & 4771 (18.39) & 1941 (13.59) & $810(5.69)$ & 109 (6.62) & $538(2.84)$ & 767 (4.64) & 8936 (9.76) \\
\hline $55-59$ & $6517(23.21)$ & 2754 (19.09) & $950(8.05)$ & $165(10.36)$ & $663(4.08)$ & 1039 (7.01) & $12,088(13.90)$ \\
\hline $60-64$ & $10,084(28.43)$ & 4571 (23.73) & 1396 (11.06) & 291 (14.93) & $974(6.13)$ & 1609 (9.52) & $18,925(18.53)$ \\
\hline $65-69$ & $11,272(33.41)$ & $5680(28.64)$ & 1402 (14.70) & 337 (19.10) & 1072 (9.61) & $1984(13.63)$ & $21,747(24.01)$ \\
\hline $70-74$ & 11,363 (38.97) & 7474 (35.91) & $1284(19.23)$ & 397 (27.08) & 913 (13.04) & 2387 (18.89) & $23,818(30.63)$ \\
\hline $75-79$ & $11,913(45.01)$ & 10,203 (41.67) & $1155(22.50)$ & $420(32.97)$ & $803(16.22)$ & 3427 (25.89) & $27,921(36.96)$ \\
\hline $80-84$ & $8803(50.44)$ & 9719 (47.01) & $742(28.44)$ & $268(34.81)$ & $537(20.07)$ & 3399 (32.25) & $23,468(42.88)$ \\
\hline $85+$ & $4884(55.61)$ & 7266 (50.88) & 369 (31.92) & $165(40.74)$ & $270(20.32)$ & 3075 (37.65) & $16,029(46.98)$ \\
\hline Total & $73,148(30.56)$ & $52,031(28.20)$ & 9269 (6.93) & $2258(14.55)$ & $6243(4.51)$ & $18,787(10.66)$ & $161,736(18.22)$ \\
\hline
\end{tabular}

CHD, coronary heart disease; CKD, chronic kidney disease; CTIN, chronic tubulointerstitial nephritis; DKD, diabetic kidney disease; GN, CKD due to glomerulonephritis; HTN hypertensive nephropathy; ON, obstructive nephropathy; Others, CKD due to other reasons.

\subsubsection{Prevalence of stroke among patients with CKD.}

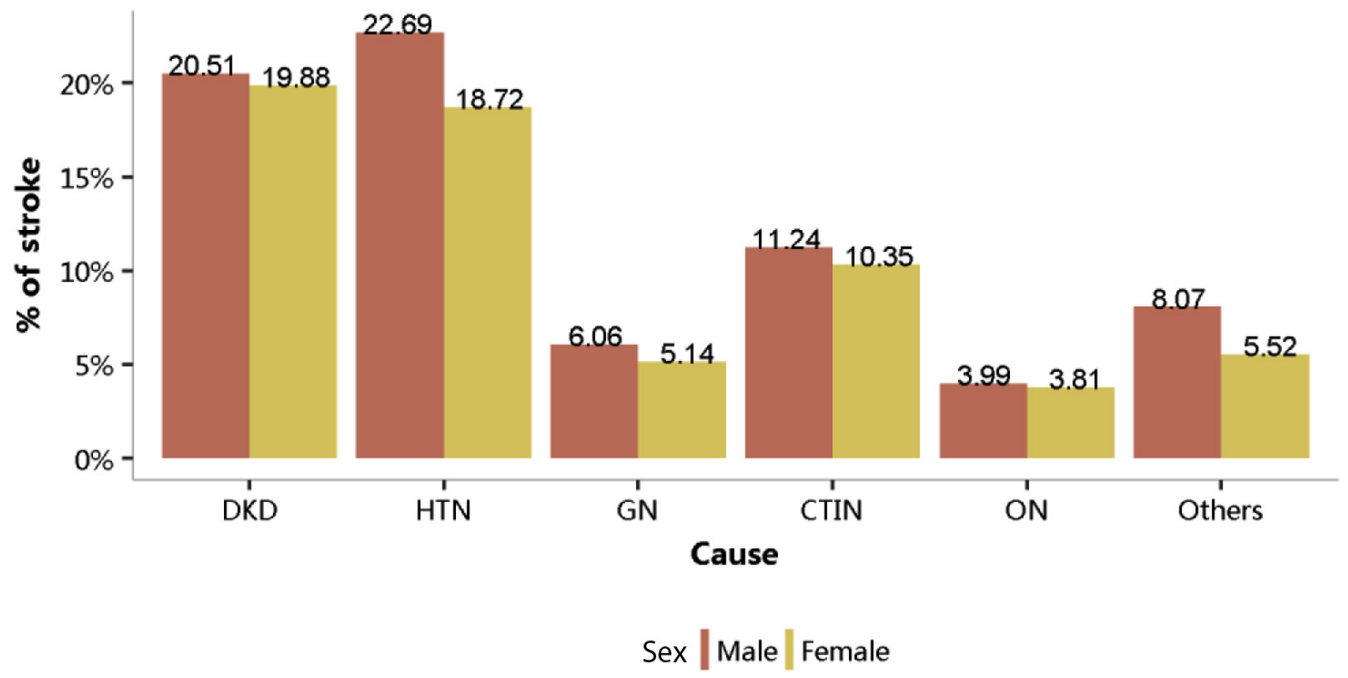

Figure 23 | Prevalence of stroke among patients with CKD, stratified by cause and sex. CKD, chronic kidney disease; CTIN, chronic tubulointerstitial nephritis; DKD, diabetic kidney disease; GN, CKD due to glomerulonephritis; HTN, hypertensive nephropathy; ON, obstructive nephropathy; Others, CKD due to other reasons.

Table 22 | Prevalence of stroke among patients with CKD stratified by cause and sex, $N$ (\%)

\begin{tabular}{|c|c|c|c|c|c|c|c|}
\hline Sex & DKD & HTN & GN & CTIN & ON & Others & Total \\
\hline Male & $28,844(20.51)$ & $25,904(22.69)$ & $4340(6.06)$ & 753 (11.24) & 3391 (3.99) & $7760(8.07)$ & $70,992(13.80)$ \\
\hline Female & $19,625(19.88)$ & $13,168(18.72)$ & $3197(5.14)$ & $913(10.35)$ & $2029(3.81)$ & $4424(5.52)$ & $43,356(11.61)$ \\
\hline Total & $48,469(20.25)$ & $39,072(21.18)$ & 7537 (5.63) & 1666 (10.73) & $5420(3.92)$ & $12,184(6.91)$ & $114,348(12.88)$ \\
\hline
\end{tabular}

CKD, chronic kidney disease; CTIN, chronic tubulointerstitial nephritis; DKD, diabetic kidney disease; GN, CKD due to glomerulonephritis; HTN, hypertensive nephropathy; ON, obstructive nephropathy; Others, CKD due to other reasons. 


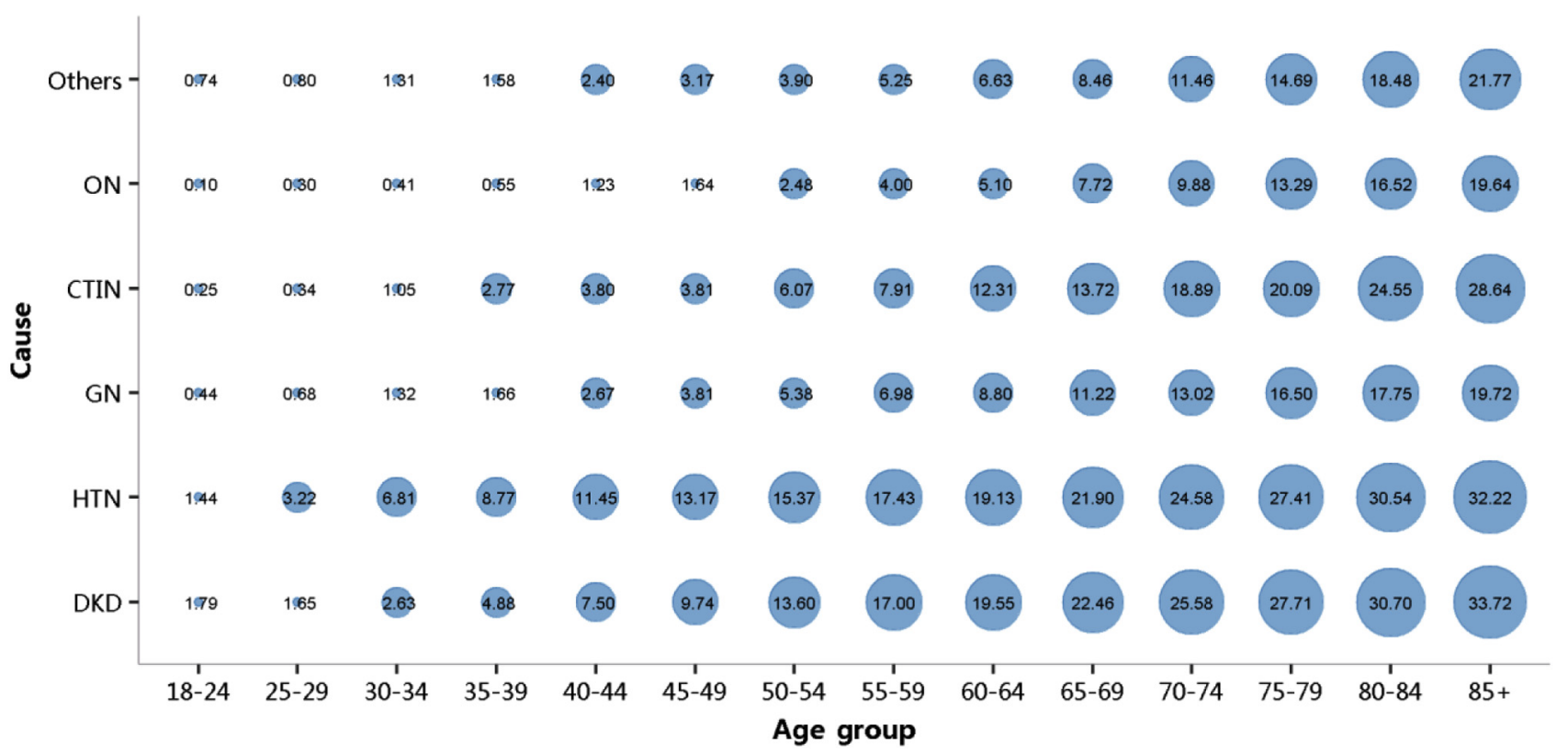

Figure 24| Prevalence of stroke among patients with CKD, stratified by cause and age. CKD, chronic kidney disease; CTIN, chronic tubulointerstitial nephritis; DKD, diabetic kidney disease; GN, CKD due to glomerulonephritis; HTN, hypertensive nephropathy; ON, obstructive nephropathy; Others, CKD due to other reasons. Point size refers to the percentage of stroke.

Table 23 | Prevalence of stroke among patients with CKD, stratified by cause and age, $N$ (\%)

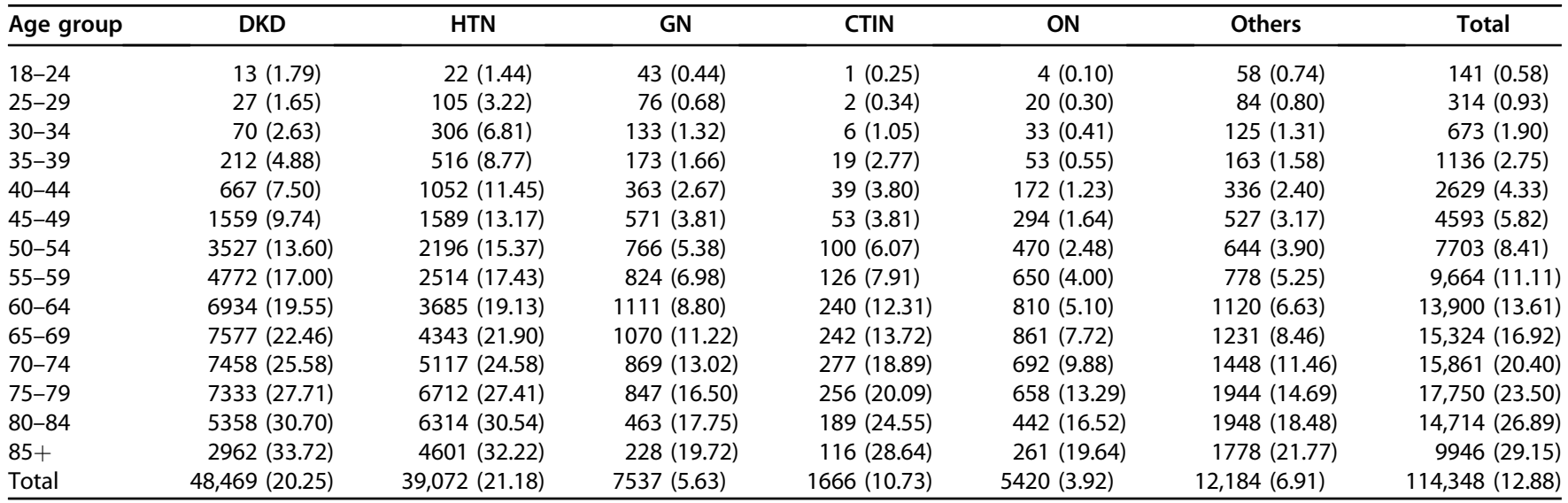

CKD, chronic kidney disease; CTIN, chronic tubulointerstitial nephritis; DKD, diabetic kidney disease; GN, CKD due to glomerulonephritis; HTN, hypertensive nephropathy; ON, obstructive nephropathy; Others, CKD due to other reasons. 


\subsubsection{Prevalence of heart failure among patients with CKD.}

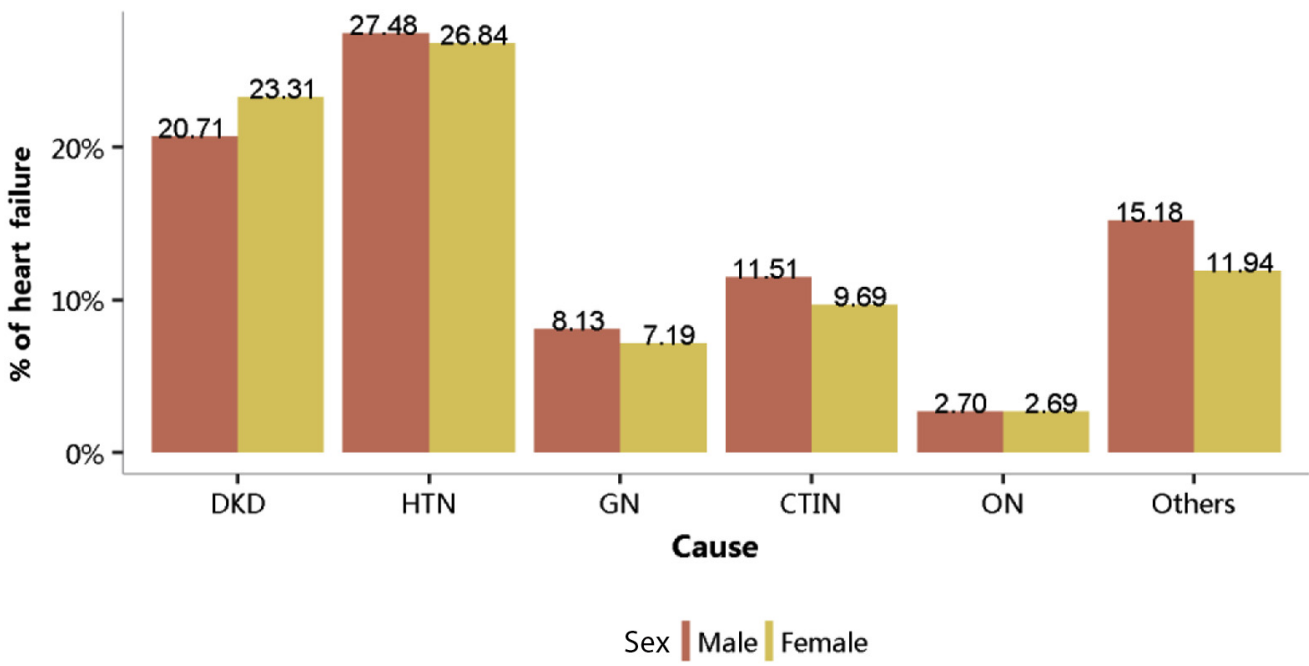

Figure 25 | Prevalence of heart failure among patients with CKD, stratified by cause and sex. CKD, chronic kidney disease; CTIN, chronic tubulointerstitial nephritis; DKD, diabetic kidney disease; GN, CKD due to glomerulonephritis; HTN, hypertensive nephropathy; ON, obstructive nephropathy; Others, CKD due to other reasons.

Table 24| Prevalence of heart failure among patients with CKD, stratified by cause and sex, $N$ (\%)

\begin{tabular}{|c|c|c|c|c|c|c|c|}
\hline Sex & DKD & HTN & GN & CTIN & ON & Others & Total \\
\hline Male & $29,123(20.71)$ & $31,373(27.48)$ & $5821(8.13)$ & 771 (11.51) & $2297(2.70)$ & $14,595(15.18)$ & $83,980(16.33)$ \\
\hline Female & 23,015 & $18,879(26.84)$ & 4474 (7.19) & 855 (9.69) & $1434(2.69)$ & 9571 (11.94) & $58,228(15.59)$ \\
\hline Total & $52,138(21.78)$ & $50,252(27.24)$ & $10,295(7.69)$ & 1626 (10.47) & $3731(2.70)$ & 24,166 (13.71) & $142,208(16.02)$ \\
\hline
\end{tabular}

CKD, chronic kidney disease; CTIN, chronic tubulointerstitial nephritis; DKD, diabetic kidney disease; GN, CKD due to glomerulonephritis; HTN, hypertensive nephropathy; ON, obstructive nephropathy; Others, CKD due to other reasons.

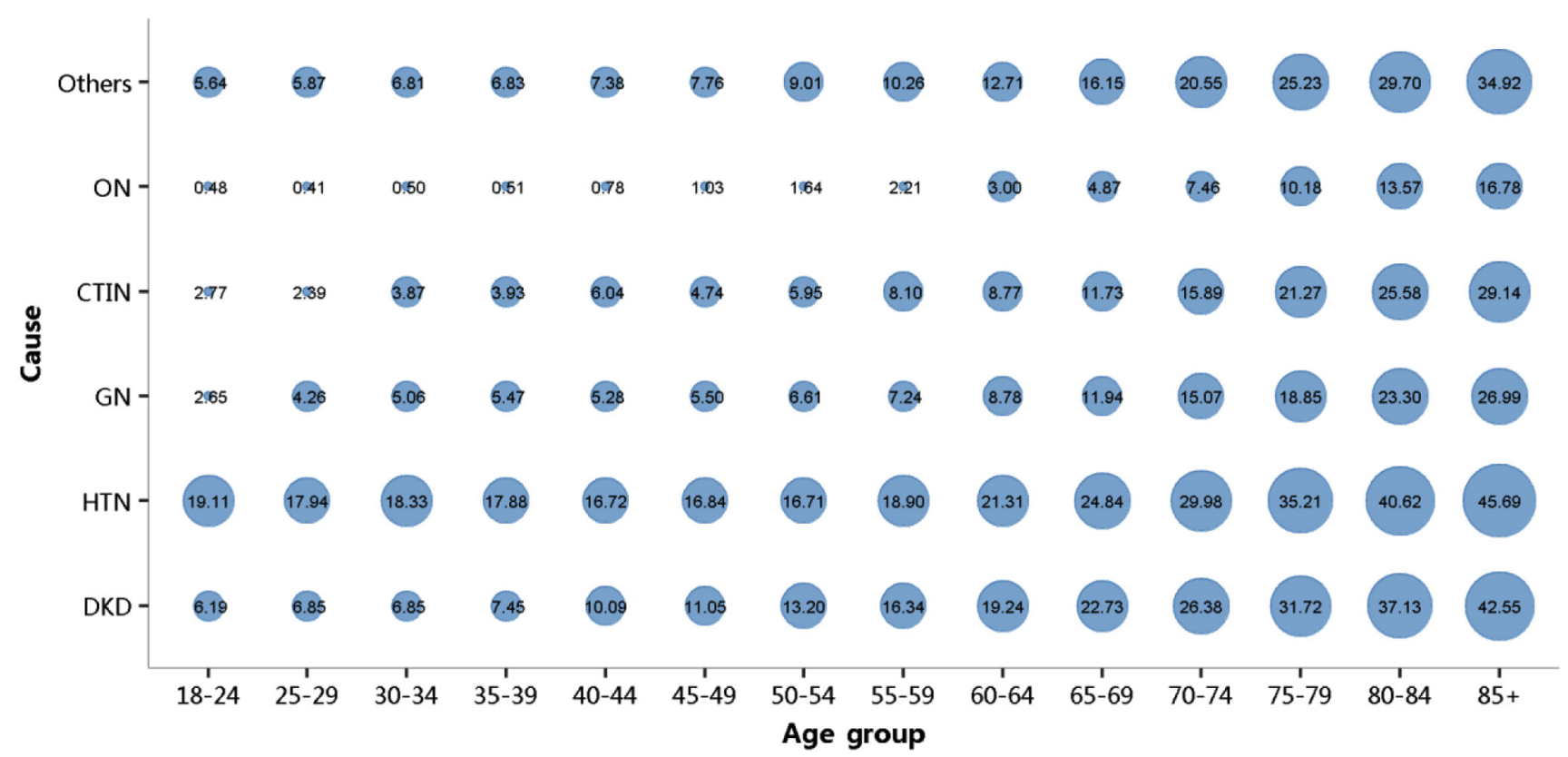

Figure 26| Prevalence of heart failure among patients with CKD, stratified by cause and age. CKD, chronic kidney disease; CTIN, chronic tubulointerstitial nephritis; DKD, diabetic kidney disease; GN, CKD due to glomerulonephritis; HTN, hypertensive nephropathy; ON, obstructive nephropathy; Others, CKD due to other reasons. Point size refers to the percentage of heart failure. 
Table 25 | Prevalence of heart failure among patients with CKD, stratified by cause and age, $N$ (\%)

\begin{tabular}{|c|c|c|c|c|c|c|c|}
\hline Age group & DKD & HTN & GN & CTIN & ON & Others & Total \\
\hline $18-24$ & 45 (6.19) & 292 (19.11) & 261 (2.65) & $11(2.77)$ & $19(0.48)$ & 445 (5.64) & $1073(4.41)$ \\
\hline $25-29$ & $112(6.85)$ & 584 (17.94) & $474(4.26)$ & $14(2.39)$ & $27(0.41)$ & 615 (5.87) & $1826(5.42)$ \\
\hline $30-34$ & $182(6.85)$ & 824 (18.33) & $511(5.06)$ & $22(3.87)$ & $41(0.50)$ & $650(6.81)$ & $2230(6.28)$ \\
\hline $35-39$ & $324(7.45)$ & 1052 (17.88) & $570(5.47)$ & 27 (3.93) & $49(0.51)$ & 707 (6.83) & $2729(6.62)$ \\
\hline $40-44$ & 897 (10.09) & $1536(16.72)$ & 717 (5.28) & $62(6.04)$ & $109(0.78)$ & $1033(7.38)$ & 4354 (7.18) \\
\hline $45-49$ & 1768 (11.05) & 2032 (16.84) & $824(5.50)$ & 66 (4.74) & 185 (1.03) & $1290(7.76)$ & $6165(7.81)$ \\
\hline $50-54$ & 3425 (13.20) & 2387 (16.71) & 941 (6.61) & $98(5.95)$ & 310 (1.64) & 1489 (9.01) & 8650 (9.44) \\
\hline $55-59$ & 4587 (16.34) & 2727 (18.90) & 855 (7.24) & $129(8.10)$ & 359 (2.21) & 1521 (10.26) & $10,178(11.70)$ \\
\hline 60-64 & 6825 (19.24) & $4106(21.31)$ & 1109 (8.78) & 171 (8.77) & 477 (3.00) & 2148 (12.71) & $14,836(14.53)$ \\
\hline $65-69$ & 7669 (22.73) & $4927(24.84)$ & 1139 (11.94) & 207 (11.73) & $543(4.87)$ & $2350(16.15)$ & 16,835 (18.58) \\
\hline 70-74 & 7691 (26.38) & $6240(29.98)$ & 1006 (15.07) & 233 (15.89) & 522 (7.46) & 2596 (20.55) & $18,288(23.52)$ \\
\hline 75-79 & 8395 (31.72) & $8621(35.21)$ & 968 (18.85) & $271(21.27)$ & $504(10.18)$ & 3339 (25.23) & $22,098(29.25)$ \\
\hline 80-84 & $6481(37.13)$ & 8399 (40.62) & $608(23.30)$ & $197(25.58)$ & 363 (13.57) & 3131 (29.70) & 19,179 (35.05) \\
\hline $85+$ & 3737 (42.55) & 6525 (45.69) & 312 (26.99) & $118(29.14)$ & $223(16.78)$ & 2852 (34.92) & $13,767(40.35)$ \\
\hline Total & $52,138(21.78)$ & $50,252(27.24)$ & 10,295 (7.69) & $1626(10.47)$ & 3731 (2.70) & $24,166(13.71)$ & $142,208(16.02)$ \\
\hline
\end{tabular}

CKD, chronic kidney disease; CTIN, chronic tubulointerstitial nephritis; DKD, diabetic kidney disease; GN, CKD due to glomerulonephritis; HTN, hypertensive nephropathy; ON, obstructive nephropathy; Others, CKD due to other reasons.

\subsubsection{Prevalence of atrial fibrillation among patients with CKD.}

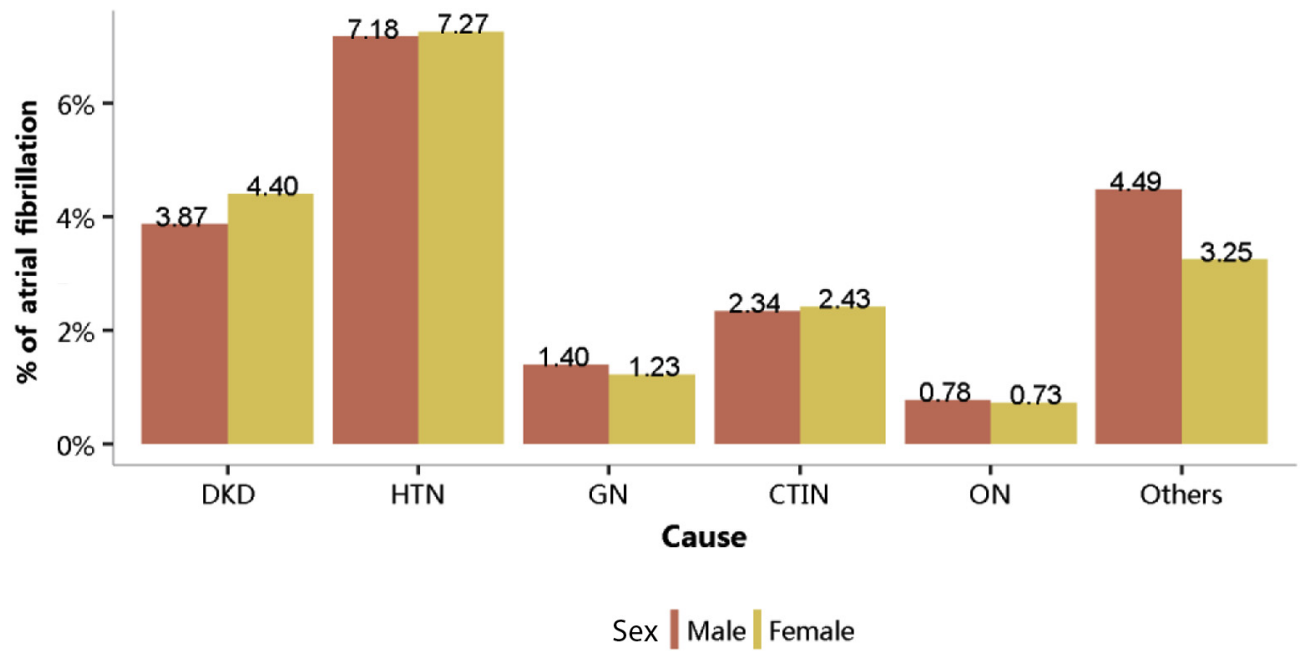

Figure 27| Prevalence of atrial fibrillation among patients with CKD, stratified by cause and sex. CKD, chronic kidney disease; CTIN, chronic tubulointerstitial nephritis; DKD, diabetic kidney disease; GN, CKD due to glomerulonephritis; HTN, hypertensive nephropathy; ON, obstructive nephropathy; Others, CKD due to other reasons.

Table 26| Prevalence of atrial fibrillation among patients with CKD, stratified by cause and sex, $N$ (\%)

\begin{tabular}{|c|c|c|c|c|c|c|c|}
\hline Sex & DKD & HTN & GN & CTIN & ON & Others & Total \\
\hline Male & 5447 (3.87) & 8195 (7.18) & $1001(1.40)$ & $157(2.34)$ & $667(0.78)$ & 4315 (4.49) & $19,782(3.85)$ \\
\hline Female & $4347(4.40)$ & $5110(7.27)$ & $763(1.23)$ & $214(2.43)$ & $388(0.73)$ & 2607 (3.25) & 13,429 (3.60) \\
\hline Total & 9794 (4.09) & $13,305(7.21)$ & $1764(1.32)$ & 371 (2.39) & 1055 (0.76) & $6922(3.93)$ & $33,211(3.74)$ \\
\hline
\end{tabular}

CKD, chronic kidney disease; CTIN, chronic tubulointerstitial nephritis; DKD, diabetic kidney disease; GN, CKD due to glomerulonephritis; HTN, hypertensive nephropathy; ON, obstructive nephropathy; Others, CKD due to other reasons. 


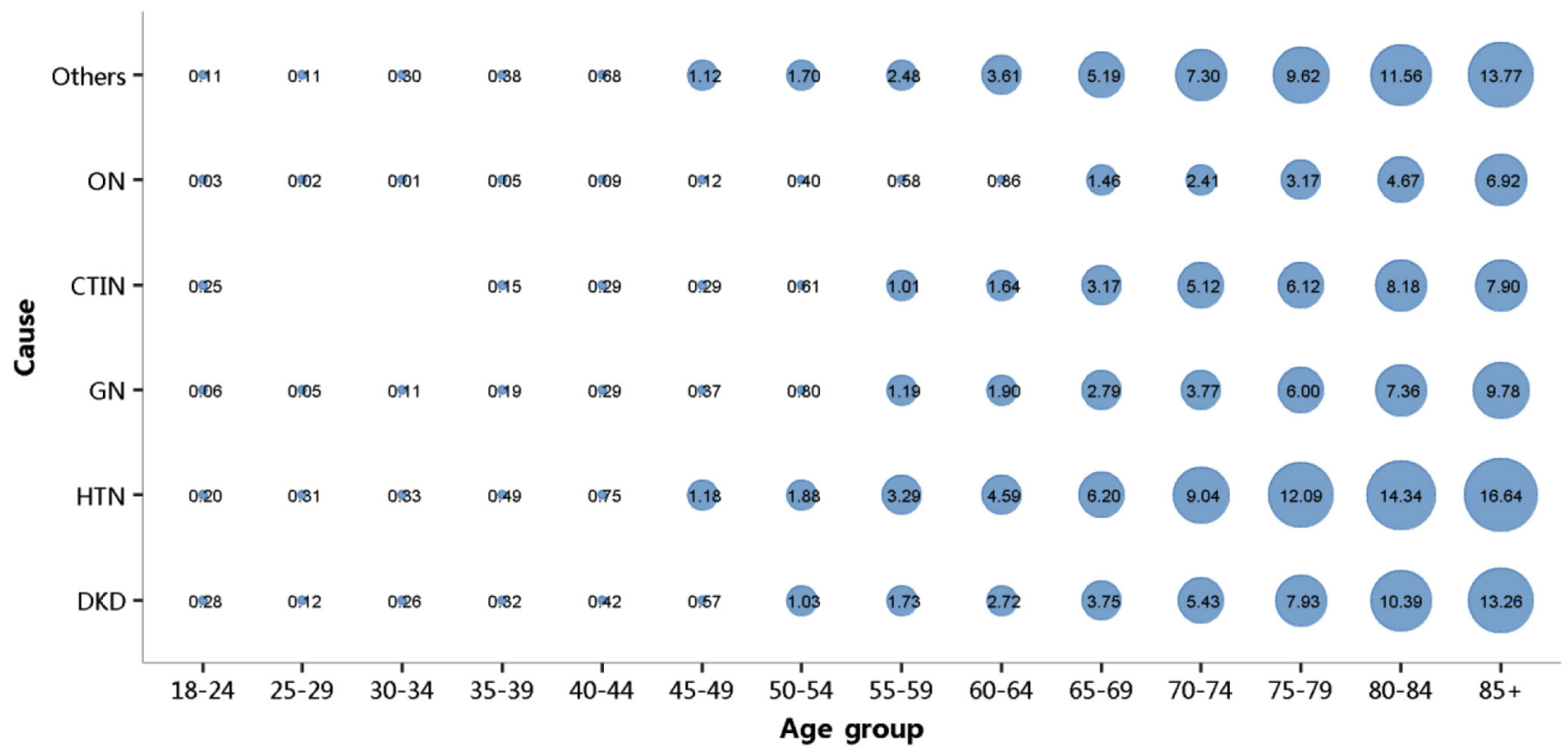

Figure 28 | Prevalence of atrial fibrillation among patients with CKD, stratified by cause and age. CKD, chronic kidney disease; CTIN, chronic tubulointerstitial nephritis; DKD, diabetic kidney disease; GN, CKD due to glomerulonephritis; HTN, hypertensive nephropathy; ON, obstructive nephropathy; Others, CKD due to other reasons. Point size refers to the percentage of atrial fibrillation.

Table 27| Prevalence of atrial fibrillation among patients with CKD, stratified by cause and age, $N$ (\%)

\begin{tabular}{|c|c|c|c|c|c|c|c|}
\hline Age group & DKD & HTN & GN & CTIN & ON & Others & Total \\
\hline $18-24$ & $2(0.28)$ & $3(0.20)$ & $6(0.06)$ & $1(0.25)$ & $1(0.03)$ & $9(0.11)$ & $22(0.09)$ \\
\hline $25-29$ & $2(0.12)$ & $10(0.31)$ & $6(0.05)$ & $0(0.00)$ & $1(0.02)$ & $11(0.11)$ & $30(0.09)$ \\
\hline $30-34$ & $7(0.26)$ & $15(0.33)$ & $11(0.11)$ & $0(0.00)$ & $1(0.01)$ & $29(0.30)$ & $63(0.18)$ \\
\hline $40-44$ & $37(0.42)$ & $69(0.75)$ & $40(0.29)$ & $3(0.29)$ & $12(0.09)$ & $95(0.68)$ & $256(0.42)$ \\
\hline $45-49$ & $92(0.57)$ & 142 (1.18) & $56(0.37)$ & $4(0.29)$ & $22(0.12)$ & $186(1.12)$ & $502(0.64)$ \\
\hline $50-54$ & 267 (1.03) & 269 (1.88) & $114(0.80)$ & $10(0.61)$ & $75(0.40)$ & $281(1.70)$ & $1,016(1.11)$ \\
\hline $65-69$ & 1264 (3.75) & $1229(6.20)$ & $266(2.79)$ & $56(3.17)$ & $163(1.46)$ & 755 (5.19) & 3733 (4.12) \\
\hline $70-74$ & $1583(5.43)$ & $1881(9.04)$ & $252(3.77)$ & $75(5.12)$ & $169(2.41)$ & $922(7.30)$ & $4882(6.28)$ \\
\hline $75-79$ & $2098(7.93)$ & 2959 (12.09) & $308(6.00)$ & $78(6.12)$ & $157(3.17)$ & $1273(9.62)$ & $6873(9.10)$ \\
\hline $80-84$ & 1813 (10.39) & 2964 (14.34) & $192(7.36)$ & $63(8.18)$ & $125(4.67)$ & $1,219(11.56)$ & 6376 (11.65) \\
\hline $85+$ & 1165 (13.26) & 2376 (16.64) & $113(9.78)$ & $32(7.90)$ & $92(6.92)$ & 1125 (13.77) & 4903 (14.37) \\
\hline Total & 9794 (4.09) & $13,305(7.21)$ & 1764 (1.32) & 371 (2.39) & $1055(0.76)$ & $6922(3.93)$ & $33,211(3.74)$ \\
\hline
\end{tabular}

CKD, chronic kidney disease; CTIN, chronic tubulointerstitial nephritis; DKD, diabetic kidney disease; GN, CKD due to glomerulonephritis; HTN, hypertensive nephropathy; ON, obstructive nephropathy; Others, CKD due to other reasons.

\subsection{Cardiovascular procedures stratified by patient group}

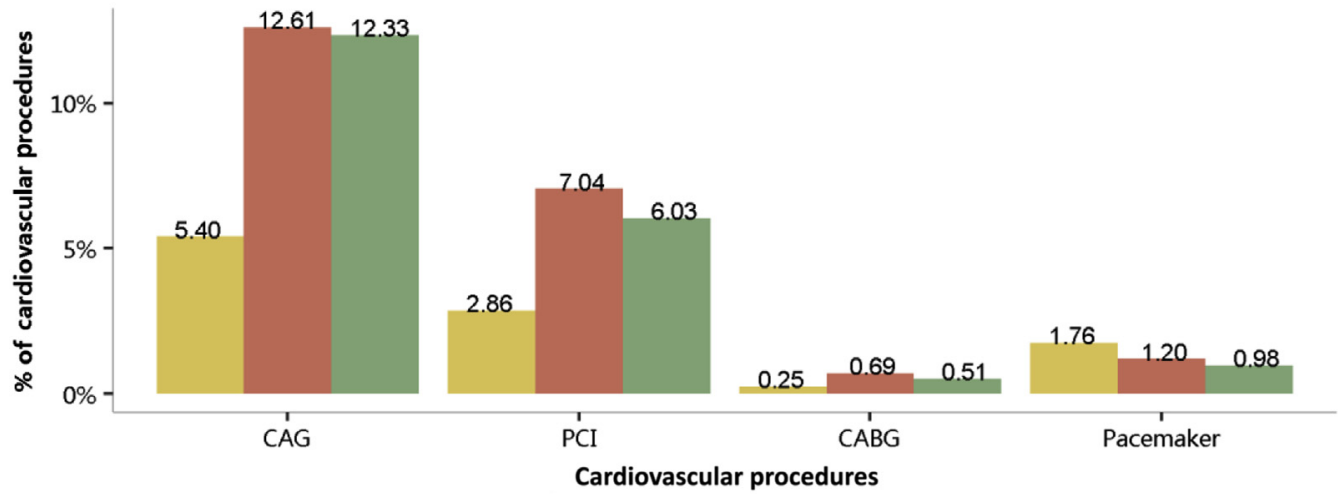

Patient groups |CKD|DM| Non-CKD

Figure 29| Cardiovascular procedures stratified by patient group. CABG, coronary artery bypass grafting; CAG, coronarography; CKD, chronic kidney disease; DM, diabetes mellitus; PCl, percutaneous coronary intervention. 
Table 28 | Cardiovascular procedures stratified by patient group, $\mathbf{N}(\%)$

\begin{tabular}{lcrrr}
\hline Patient group & CAG & \multicolumn{1}{c}{ PCI } & \multicolumn{1}{c}{ CABG } & \multicolumn{1}{c}{ Pacemaker } \\
\hline CKD & $16,071(5.40)$ & $8515(2.86)$ & $737(0.25)$ & $5247(1.76)$ \\
DM & $100,573(12.61)$ & $56,195(7.04)$ & $5540(0.69)$ & $9603(1.20)$ \\
Non-CKD & $438,820(12.33)$ & $214,619(6.03)$ & $18,210(0.51)$ & $34,747(0.98)$ \\
\hline
\end{tabular}

CABG, coronary artery bypass grafting; CAG, coronarography; CKD, chronic kidney disease; DM, diabetes mellitus; PCl, percutaneous coronary intervention.

\subsubsection{Cardiovascular procedures: coronarography.}

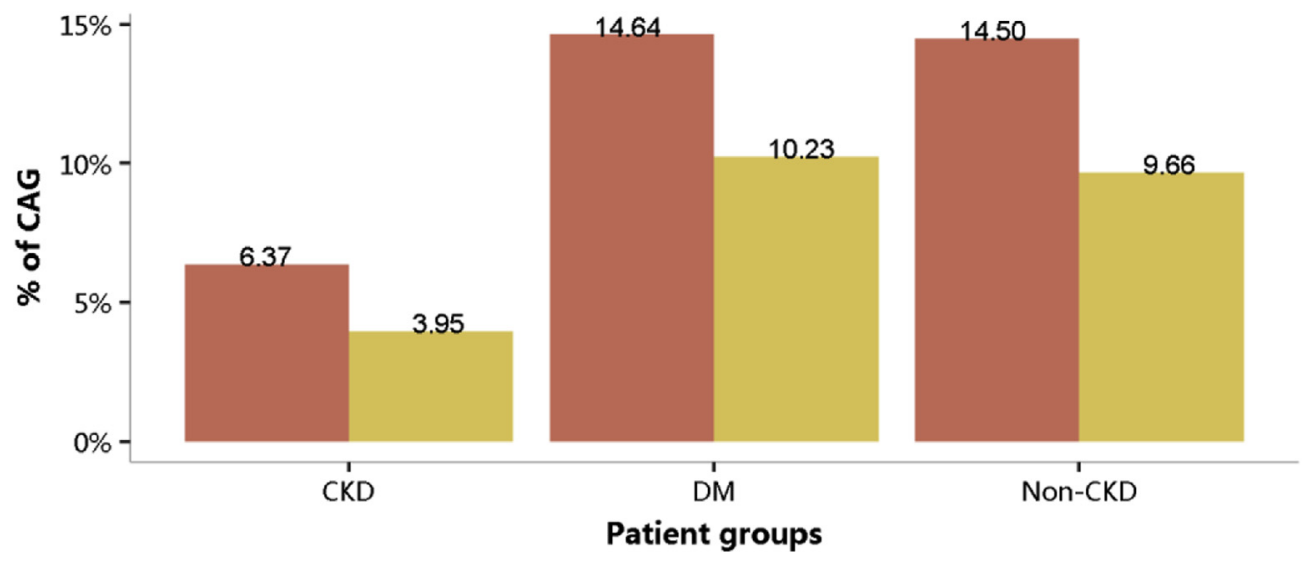

$$
\text { Sex |Male| Female }
$$

Figure 30| Cardiovascular procedures: CAG, stratified by sex. CAG, coronarography; CKD, chronic kidney disease; DM, diabetes mellitus.

Table 29| Cardiovascular procedures: CAG, stratified by sex, $N$ (\%)

\begin{tabular}{lrrr}
\hline Sex & CKD & DM & Non-CKD \\
\hline Male & $11,391(6.37)$ & $62,961(14.64)$ & $284,564(14.50)$ \\
Female & $4680(3.95)$ & $37,612(10.23)$ & $154,256(9.66)$ \\
Total & $16,071(5.40)$ & $100,573(12.61)$ & $438,820(12.33)$ \\
\hline
\end{tabular}

CAG, coronarography; CKD, chronic kidney disease; DM, diabetes mellitus.

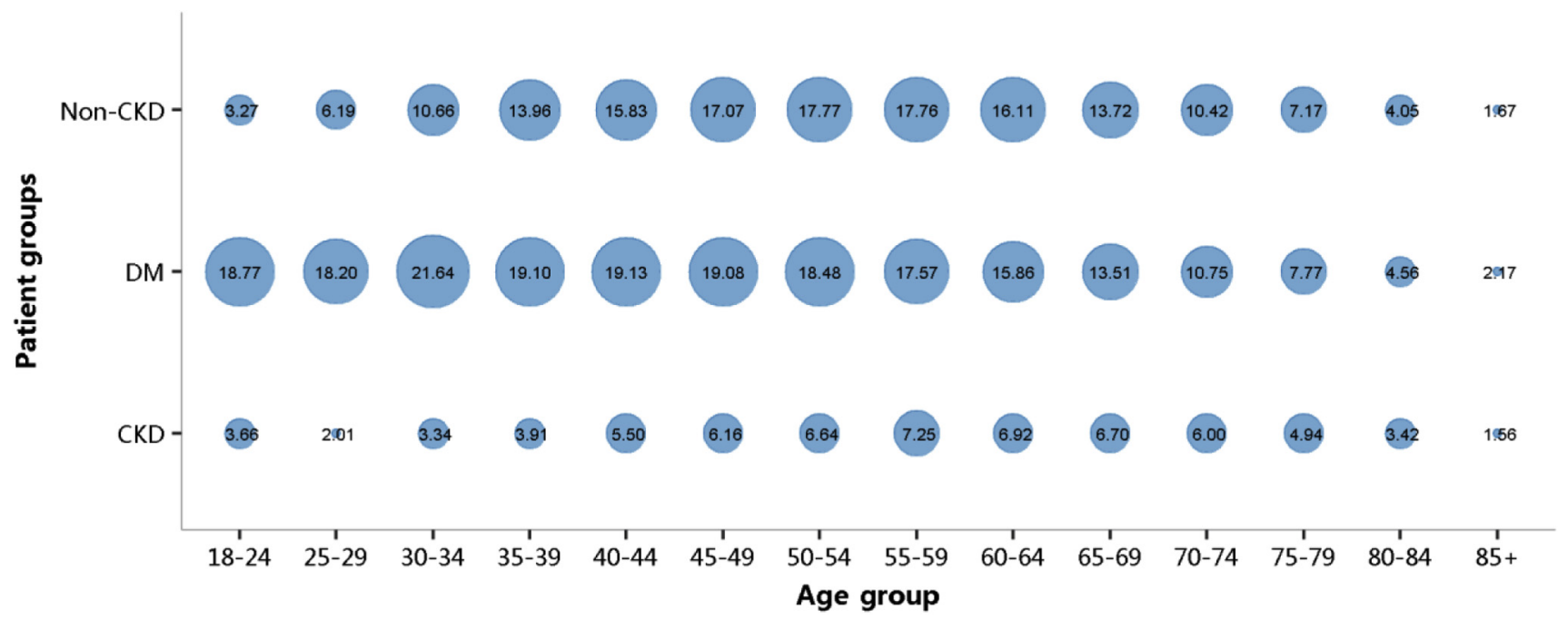

Figure 31 | Cardiovascular procedures: CAG, stratified by age. CAG, coronarography; CKD, chronic kidney disease; DM, diabetes mellitus. Point size refers to the percentage of CAG. 
Table 30| Cardiovascular procedures: CAG, stratified by age, $\mathbf{N}(\%)$

\begin{tabular}{lrrr}
\hline Age group & \multicolumn{1}{c}{ CKD } & DM & \multicolumn{1}{c}{ Non-CKD } \\
\hline $18-24$ & $46(3.66)$ & $52(18.77)$ & $299(3.27)$ \\
$25-29$ & $45(2.01)$ & $117(18.20)$ & $904(6.19)$ \\
$30-34$ & $103(3.34)$ & $344(21.64)$ & $2396(10.66)$ \\
$35-39$ & $167(3.91)$ & $853(19.10)$ & $6099(13.96)$ \\
$40-44$ & $444(5.50)$ & $2602(19.13)$ & $16,290(15.83)$ \\
$45-49$ & $789(6.16)$ & $5882(19.08)$ & $32,472(17.07)$ \\
$50-54$ & $1343(6.64)$ & $11,843(18.48)$ & $54,829(17.77)$ \\
$55-59$ & $1789(7.25)$ & $15,543(17.57)$ & $69,723(17.76)$ \\
$60-64$ & $2459(6.92)$ & $19,996(15.86)$ & $85,834(16.11)$ \\
$65-69$ & $2613(6.70)$ & $17,680(13.51)$ & $71,193(13.72)$ \\
$70-74$ & $2422(6.00)$ & $8517(10.78)$ & $50,623(10.42)$ \\
$75-79$ & $2221(4.94)$ & $3239(4.56)$ & $32,649(7.17)$ \\
$80-84$ & $1250(3.42)$ & $730(2.17)$ & $12,686(4.05)$ \\
$85+$ & $380(1.56)$ & $100,573(12.61)$ & $2823(1.67)$ \\
Total & $16,071(5.40)$ &
\end{tabular}

CAG, coronarography; CKD, chronic kidney disease; DM, diabetes mellitus.

\subsubsection{Cardiovascular procedures: percutaneous coronary intervention.}

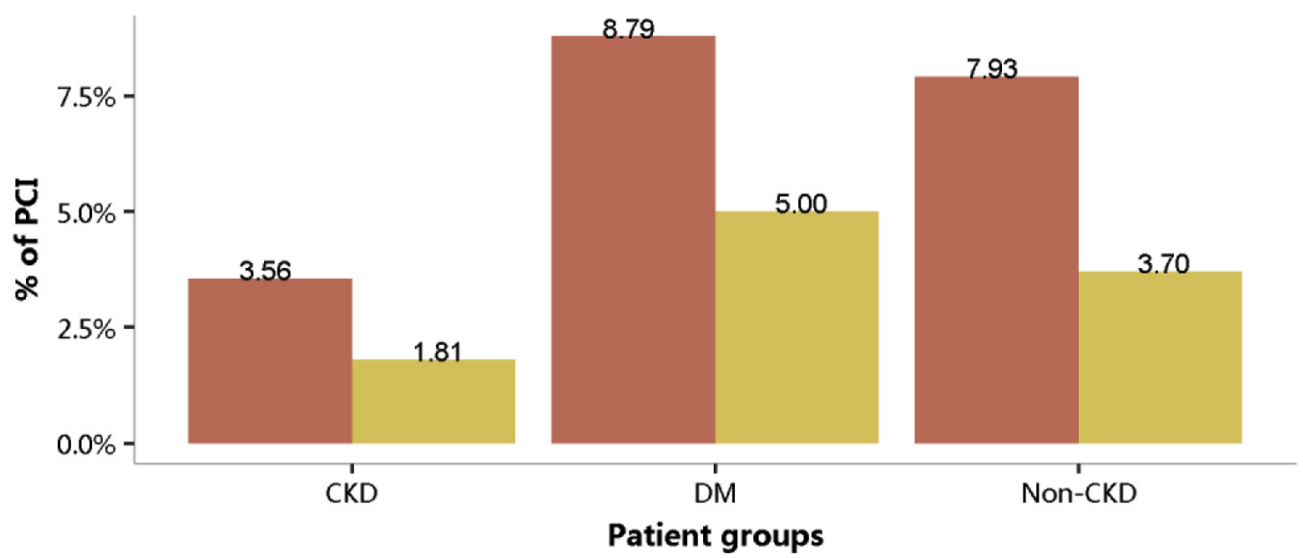

Sex |Male | Female

Figure 32 | Cardiovascular procedures: PCI, stratified by sex. CKD, chronic kidney disease; DM, diabetes mellitus; PCl, percutaneous coronary intervention.

Table 31 | Cardiovascular procedures: $\mathrm{PCl}$, stratified by sex, $\mathrm{N}(\%)$

\begin{tabular}{lcrr}
\hline Sex & CKD & DM & Non-CKD \\
\hline Male & $6370(3.56)$ & $37,799(8.79)$ & $155,542(7.93)$ \\
Female & $2145(1.81)$ & $18,396(5.00)$ & $59,077(3.70)$ \\
Total & $8515(2.86)$ & $56,195(7.04)$ & $214,619(6.03)$ \\
\hline
\end{tabular}

CKD, chronic kidney disease; DM, diabetes mellitus; $\mathrm{PCl}$, percutaneous coronary intervention. 


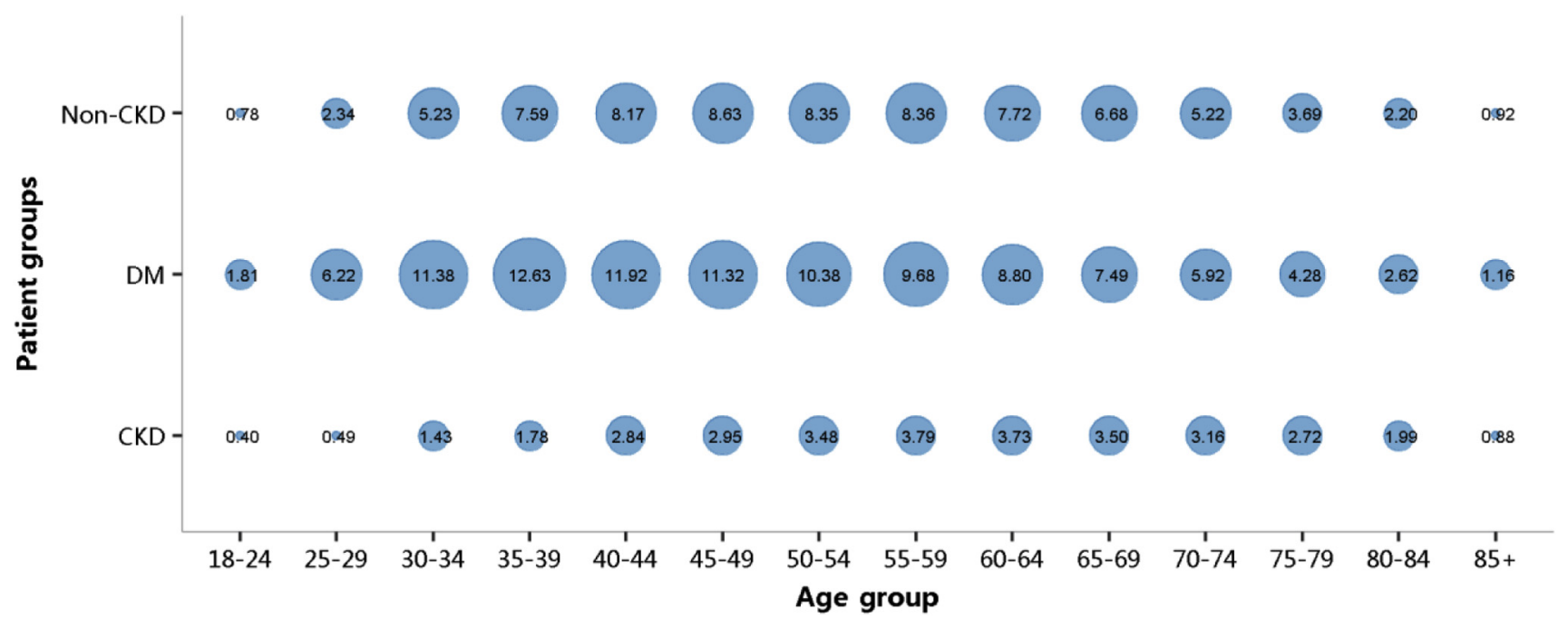

Figure 33 | Cardiovascular procedures: PCI, stratified by age. CKD, chronic kidney disease; DM, diabetes mellitus; $\mathrm{PCl}$, percutaneous coronary intervention. Point size refers to the percentage of $\mathrm{PCl}$.

Table 32 | Cardiovascular procedures: PCl, stratified by age, $N$ (\%)

\begin{tabular}{lrrr}
\hline Age group & \multicolumn{1}{c}{ CKD } & \multicolumn{1}{c}{ DM } & Non-CKD \\
\hline $18-24$ & $5(0.40)$ & $5(1.81)$ & $71(0.78)$ \\
$25-29$ & $11(0.49)$ & $40(6.22)$ & $342(2.34)$ \\
$30-34$ & $44(1.43)$ & $181(11.38)$ & $1175(5.23)$ \\
$35-39$ & $76(1.78)$ & $564(12.63)$ & $3318(7.59)$ \\
$40-44$ & $229(2.84)$ & $1622(11.92)$ & $8405(8.17)$ \\
$45-49$ & $378(2.95)$ & $3489(11.32)$ & $16,416(8.63)$ \\
$50-54$ & $704(3.48)$ & $6650(10.38)$ & $25,745(8.35)$ \\
$55-59$ & $936(3.79)$ & $8561(9.68)$ & $32,824(8.36)$ \\
$60-64$ & $1326(3.73)$ & $11,091(8.80)$ & $41,098(7.72)$ \\
$65-69$ & $1367(3.50)$ & $9796(7.49)$ & $34,668(6.68)$ \\
$70-74$ & $1275(3.16)$ & $7252(5.92)$ & $25,338(5.22)$ \\
$75-79$ & $1221(2.72)$ & $4694(4.28)$ & $16,791(3.69)$ \\
$80-84$ & $727(1.99)$ & $391(1.16)$ & $6873(2.20)$ \\
$85+$ & $216(0.88)$ & $56,195(7.04)$ & $1555(0.92)$ \\
Total & $8515(2.86)$ & & $214,619(6.03)$ \\
\hline
\end{tabular}

CKD, chronic kidney disease; DM, diabetes mellitus; PCl, percutaneous coronary intervention.

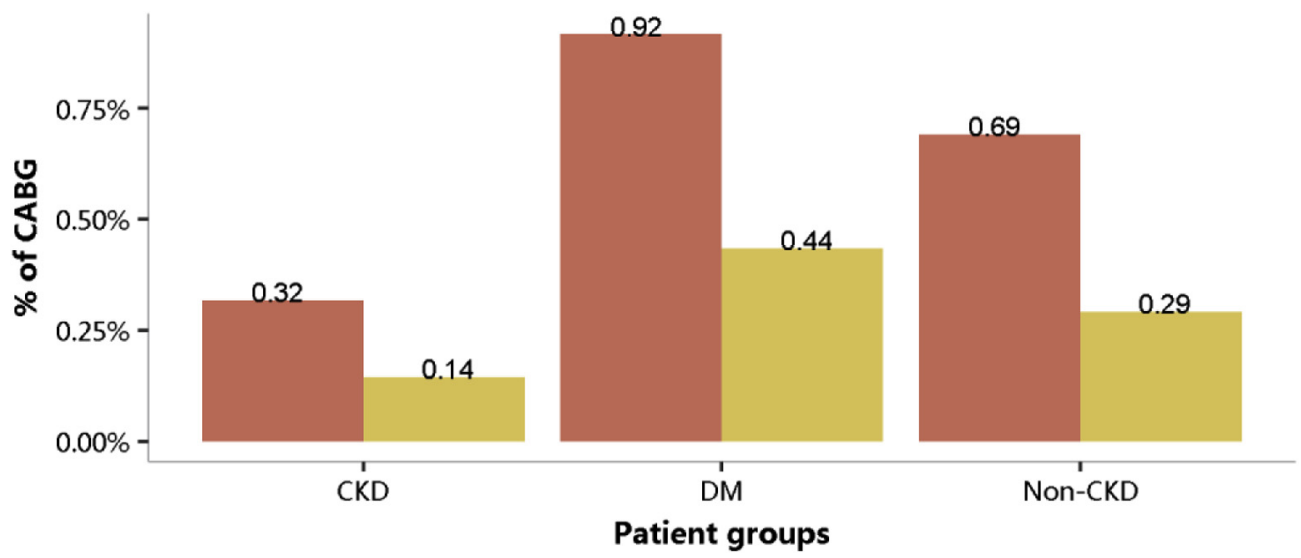

$$
\text { Sex | Male | Female }
$$

Figure 34 | Cardiovascular procedures: CABG, stratified by sex. CABG, coronary artery bypass grafting; CKD, chronic kidney disease; DM, diabetes mellitus. 
Table 33 | Cardiovascular procedures: CABG, stratified by sex, $N$ (\%)

\begin{tabular}{lcrr}
\hline Sex & CKD & DM & Non-CKD \\
\hline Male & $566(0.32)$ & $3941(0.92)$ & $13,563(0.69)$ \\
Female & $171(0.14)$ & $1599(0.44)$ & $4647(0.29)$ \\
Total & $737(0.25)$ & $5540(0.69)$ & $18,210(0.51)$ \\
\hline
\end{tabular}

CABG, coronary artery bypass grafting; CKD, chronic kidney disease; DM, diabetes mellitus.

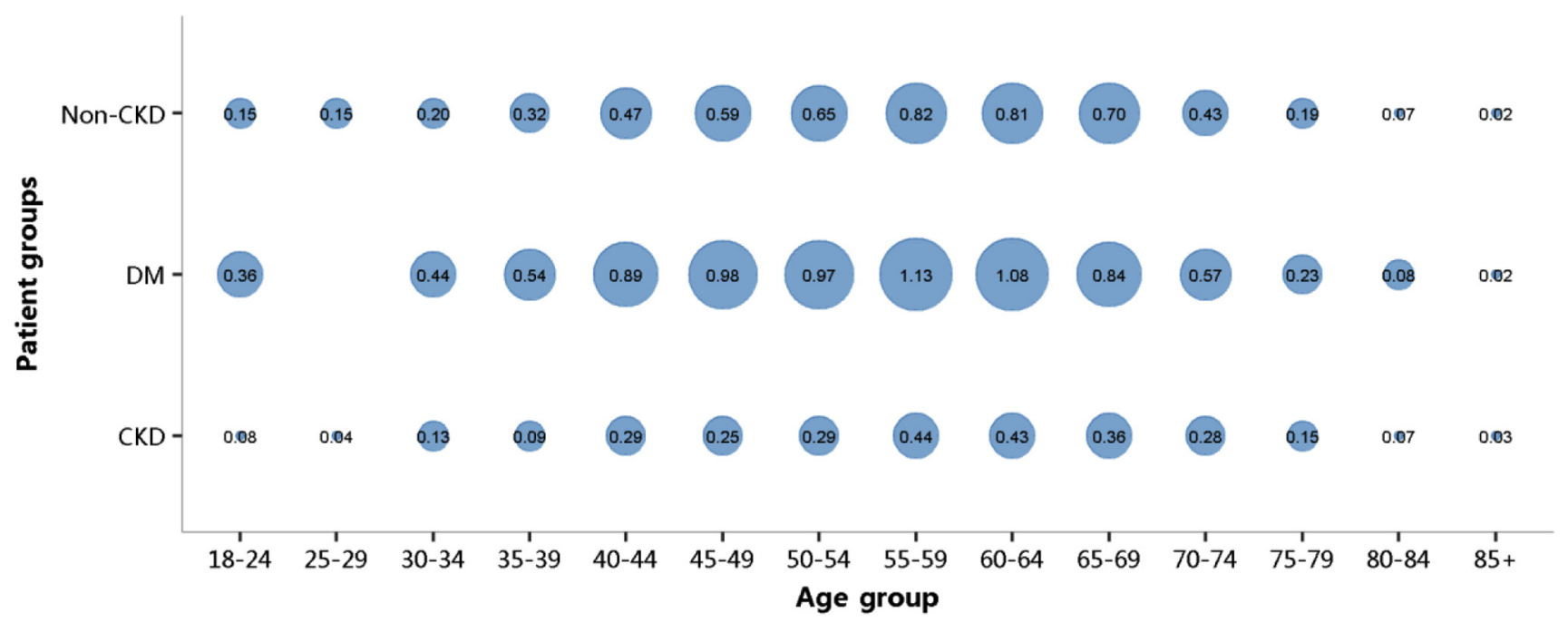

Figure 35 | Cardiovascular procedures: CABG, stratified by age. CABG, coronary artery bypass grafting; CKD, chronic kidney disease; DM, diabetes mellitus. Point size refers to the percentage of CABG.

2.3.3 Cardiovascular procedures: coronary artery bypass grafting.

Table 34 | Cardiovascular procedures: CABG, stratified by age, $N$ (\%)

\begin{tabular}{lrrr}
\hline Age group & \multicolumn{1}{c}{ CKD } & \multicolumn{1}{c}{ DM } & Non-CKD \\
\hline $18-24$ & $1(0.08)$ & $1(0.36)$ & $14(0.15)$ \\
$25-29$ & $1(0.04)$ & $0(0.00)$ & $22(0.15)$ \\
$30-34$ & $4(0.13)$ & $7(0.44)$ & $45(0.20)$ \\
$35-39$ & $4(0.09)$ & $24(0.54)$ & $142(0.32)$ \\
$40-44$ & $23(0.29)$ & $121(0.89)$ & $487(0.47)$ \\
$45-49$ & $32(0.25)$ & $303(0.98)$ & $1124(0.59)$ \\
$50-54$ & $58(0.29)$ & $619(0.97)$ & $2019(0.65)$ \\
$55-59$ & $108(0.44)$ & $999(1.13)$ & $3209(0.82)$ \\
$60-64$ & $152(0.43)$ & $1357(1.08)$ & $4300(0.81)$ \\
$65-69$ & $140(0.36)$ & $1094(0.84)$ & $3632(0.70)$ \\
$70-74$ & $113(0.28)$ & $698(0.57)$ & $2081(0.43)$ \\
$75-79$ & $69(0.15)$ & $254(0.23)$ & $882(0.19)$ \\
$80-84$ & $24(0.07)$ & $57(0.08)$ & $6(0.02)$ \\
$85+$ & $8(0.03)$ & $5540(0.69)$ & $215(0.07)$ \\
Total & $737(0.25)$ & & $38(0.02)$ \\
\hline
\end{tabular}

CABG, coronary artery bypass grafting; CKD, chronic kidney disease; DM, diabetes mellitus. 


\subsubsection{Cardiovascular procedures: pacemaker.}

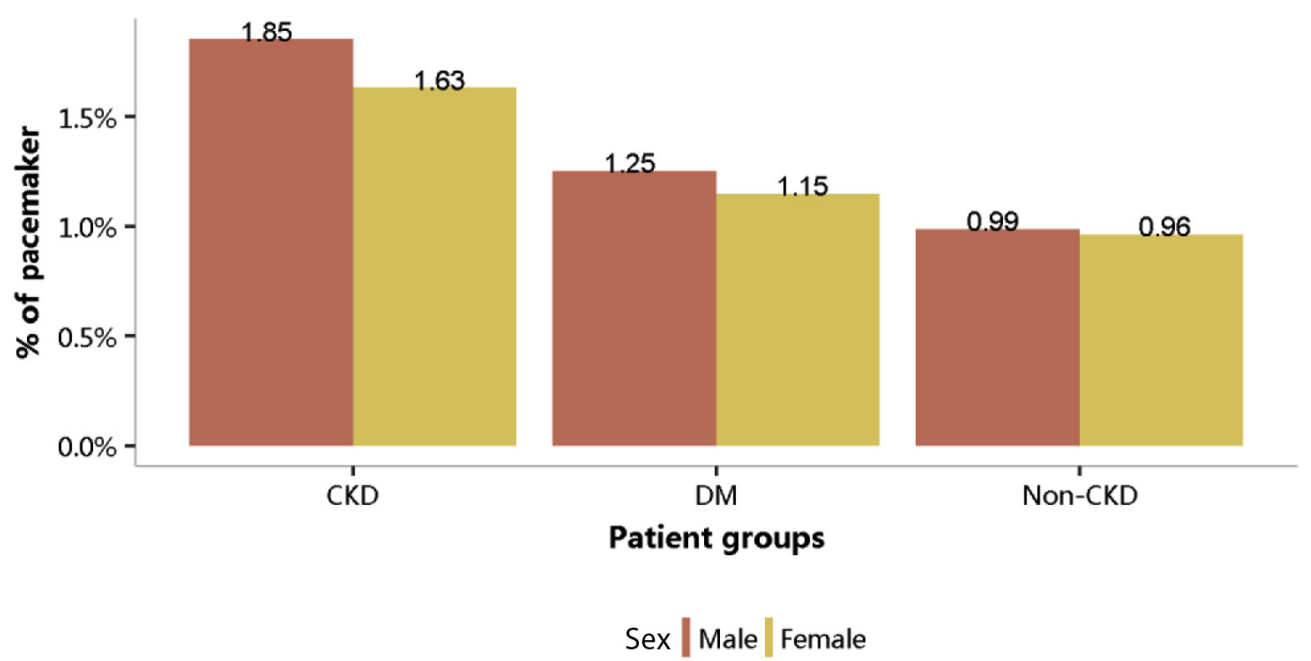

Figure 36| Cardiovascular procedures: pacemaker, stratified by sex. CKD, chronic kidney disease; DM, diabetes mellitus.

Table 35 | Cardiovascular procedures: pacemaker, stratified by sex, $N$ (\%)

\begin{tabular}{lccr}
\hline Sex & CKD & DM & Non-CKD \\
\hline Male & $3314(1.85)$ & $5385(1.25)$ & $19,364(0.99)$ \\
Female & $1933(1.63)$ & $4218(1.15)$ & $15,383(0.96)$ \\
Total & $5247(1.76)$ & $9603(1.20)$ & $34,747(0.98)$ \\
\hline
\end{tabular}

CKD, chronic kidney disease; DM, diabetes mellitus.

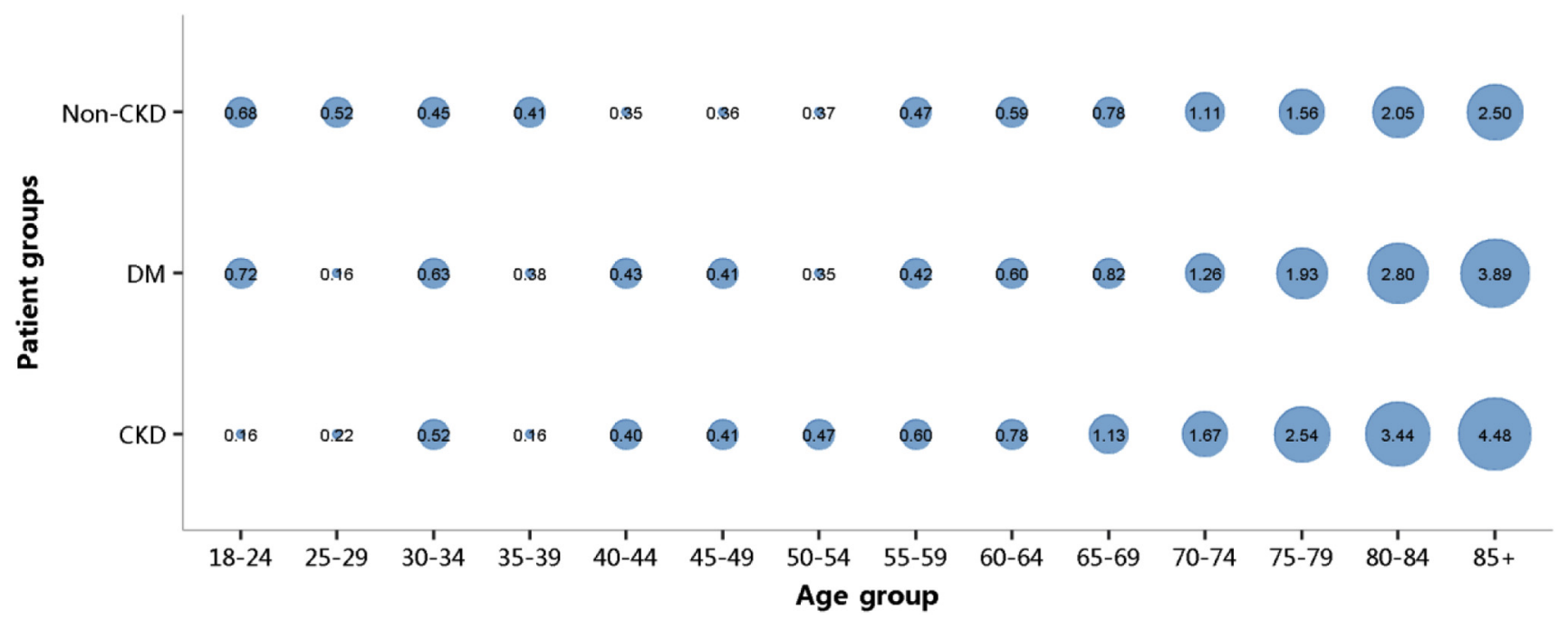

Figure 37| Cardiovascular procedures: pacemaker, stratified by age. CKD, chronic kidney disease; DM, diabetes mellitus. Point size refers to the percentage of pacemaker. 
Table 36 | Cardiovascular procedures: pacemaker, stratified by age, $N$ (\%)

\begin{tabular}{lrrr}
\hline Age group & \multicolumn{1}{c}{ CKD } & \multicolumn{1}{c}{ DM } & Non-CKD \\
\hline $18-24$ & $2(0.16)$ & $2(0.72)$ & $62(0.68)$ \\
$25-29$ & $5(0.22)$ & $1(0.16)$ & $76(0.52)$ \\
$30-34$ & $16(0.52)$ & $10(0.63)$ & $102(0.45)$ \\
$35-39$ & $7(0.16)$ & $17(0.38)$ & $181(0.41)$ \\
$40-44$ & $32(0.40)$ & $58(0.43)$ & $360(0.35)$ \\
$45-49$ & $52(0.41)$ & $125(0.41)$ & $679(0.36)$ \\
$50-54$ & $96(0.47)$ & $223(0.35)$ & $1141(0.37)$ \\
$55-59$ & $148(0.60)$ & $375(0.42)$ & $1831(0.47)$ \\
$60-64$ & $278(0.78)$ & $756(0.60)$ & $3131(0.59)$ \\
$65-69$ & $442(1.13)$ & $1075(0.82)$ & $4056(0.78)$ \\
$70-74$ & $676(1.67)$ & $1538(1.26)$ & $5396(1.11)$ \\
$75-79$ & $1143(2.54)$ & $2120(1.93)$ & $7091(1.56)$ \\
$80-84$ & $1257(3.44)$ & $1993(2.80)$ & $6426(2.05)$ \\
$85+$ & $1093(4.48)$ & $9603(1.20)$ & $4215(2.50)$ \\
Total & $5247(1.76)$ & $34,747(0.98)$ \\
\hline
\end{tabular}

CKD, chronic kidney disease; DM, diabetes mellitus.

\subsection{Cardiovascular procedures among patients with CKD}

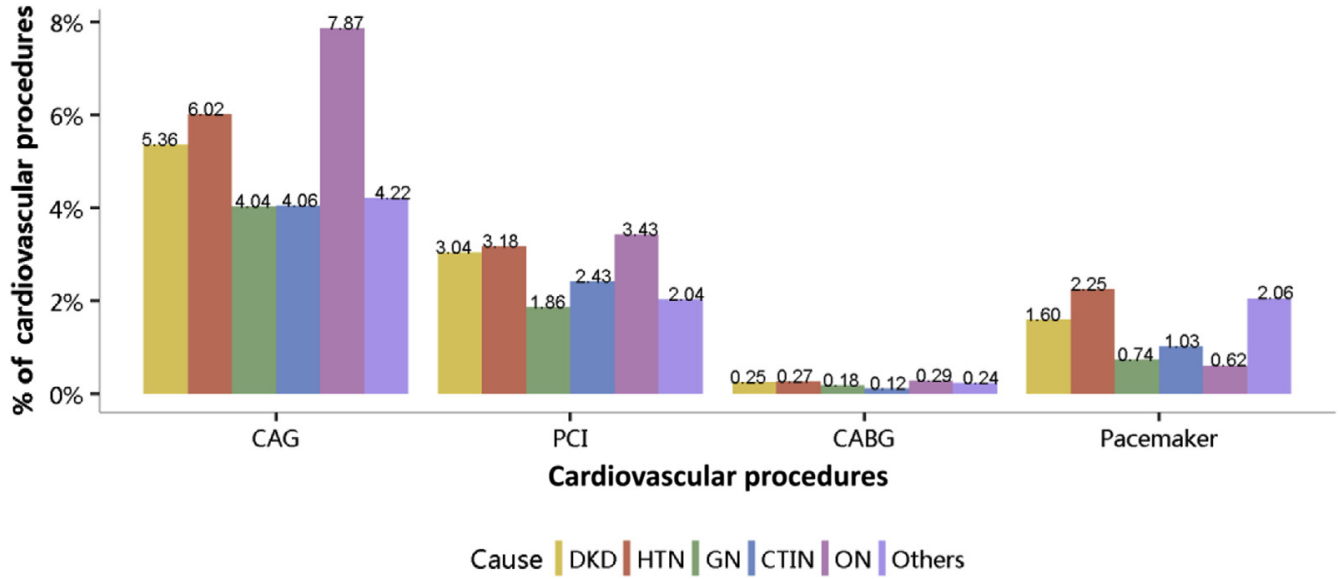

Figure 38 | Cardiovascular procedures among patients with CKD. CABG, coronary artery bypass grafting; CAG, coronarography; CKD, chronic kidney disease; CTIN, chronic tubulointerstitial nephritis; DKD, diabetic kidney disease; GN, CKD due to glomerulonephritis; HTN, hypertensive nephropathy; ON, obstructive nephropathy; Others, CKD due to other reasons; $\mathrm{PCl}$, percutaneous coronary intervention

Table 37| Cardiovascular procedures among patients with CKD, N (\%)

\begin{tabular}{|c|c|c|c|c|c|}
\hline Cause & CAG & $\mathrm{PCl}$ & CABG & Pacemaker & Total \\
\hline DKD & 6357 (5.36) & 3606 (3.04) & $293(0.25)$ & $1894(1.60)$ & $12,150(2.56)$ \\
\hline HTN & $5879(6.02)$ & 3103 (3.18) & $261(0.27)$ & $2200(2.25)$ & $11,443(2.93)$ \\
\hline $\mathrm{GN}$ & $888(4.04)$ & 409 (1.86) & $40(0.18)$ & $162(0.74)$ & 1499 (1.70) \\
\hline ON & 998 (7.87) & $435(3.43)$ & $37(0.29)$ & $78(0.62)$ & $1548(3.05)$ \\
\hline Others & $1780(4.22)$ & $861(2.04)$ & $101(0.24)$ & $870(2.06)$ & $3612(2.14)$ \\
\hline Total & $16,071(5.40)$ & $8515(2.86)$ & $737(0.25)$ & 5247 (1.76) & $30,570(2.57)$ \\
\hline
\end{tabular}

CABG, coronary artery bypass grafting; CAG, coronarography; CKD, chronic kidney disease; CTIN, chronic tubulointerstitial nephritis; DKD, diabetic kidney disease; GN, CKD due to glomerulonephritis; HTN, hypertensive nephropathy; ON, obstructive nephropathy; Others, CKD due to other reasons; PCl, percutaneous coronary intervention. 


\title{
Chapter 3: Health care resource utilization of hospitalized patients with CKD
}

\author{
Kunhao Bai ${ }^{1}$, Jianyan Long ${ }^{2}$, Chao Yang ${ }^{3}$, Lanxia Gan ${ }^{4}$, Jinwei Wang ${ }^{3}$ and Haibo Wang ${ }^{4,5}$ \\ ${ }^{1}$ Department of Endoscopy, Sun Yat-sen University Cancer Center, State Key Laboratory of Oncology in South China, Collaborative \\ Innovation Center for Cancer Medicine, Guangzhou, China; ${ }^{2}$ Clinical Trial Unit, The First Affiliated Hospital of Sun Yat-sen \\ University, Guangzhou, China; ${ }^{3}$ Renal Division, Department of Medicine, Peking University First Hospital; Peking University \\ Institute of Nephrology, Beijing, China; ${ }^{4}$ China Standard Medical Information Research Center, Shenzhen, China; and ${ }^{5}$ Center for \\ Data Science in Health and Medicine, Peking University, Beijing, China
}

This chapter focuses on the medical expenditure and the length of stay (LOS) of inpatients with CKD, which are both important indicators for health care resource utilization. Due to the high skewness of cost and LOS, the results are displayed as the median and interquartile range (IQR), and mean and $\mathrm{SD}$ are also provided.

In 2015, the total medical expenditure of inpatients with CKD was 23,780 million renminbi $(\mathrm{RMB})(\sim 3,430$ million US dollars as of October 2018), accounting for $6.34 \%$ of the overall costs in the dataset, while the number of inpatients with CKD accounted for $4.80 \%$ of the overall hospitalized population (Table 38). The median cost of inpatients with CKD was 14,965 (IQR: 8,302-28,282) $\mathrm{RMB}$, which was higher than for those with diabetes
(13,749 [IQR: 7,666-24,955)] RMB) and without CKD (11,219 [IQR: 5,867-18,556] RMB) (Table 39). The trends were consistent across age-sex subgroups and across different types of health insurance (Figures 39-41 and Tables 39-44). Furthermore, compared with other comorbidities, patients with CKD and heart failure had relatively higher medical costs $(43,196 \mathrm{RMB}$ per person per year [PPPY]) (Table 38).

The median LOS of inpatients with CKD was 13 (IQR: 822) days, which was higher than that of patients with diabetes (11 [IQR: 7-19] days) and inpatients without CKD (8 [IQR: 5-14] days) (Tables 45 and 46). The trends were consistent across age-sex subgroups and across types of health insurance as well (Figures 42-44 and Tables 46-51).

\subsection{Costs}

3.1.1 Overall medical care costs stratified by CKD, DM, and heart failure.

Table 38| Overall medical care costs, stratified by CKD, DM, and heart failure

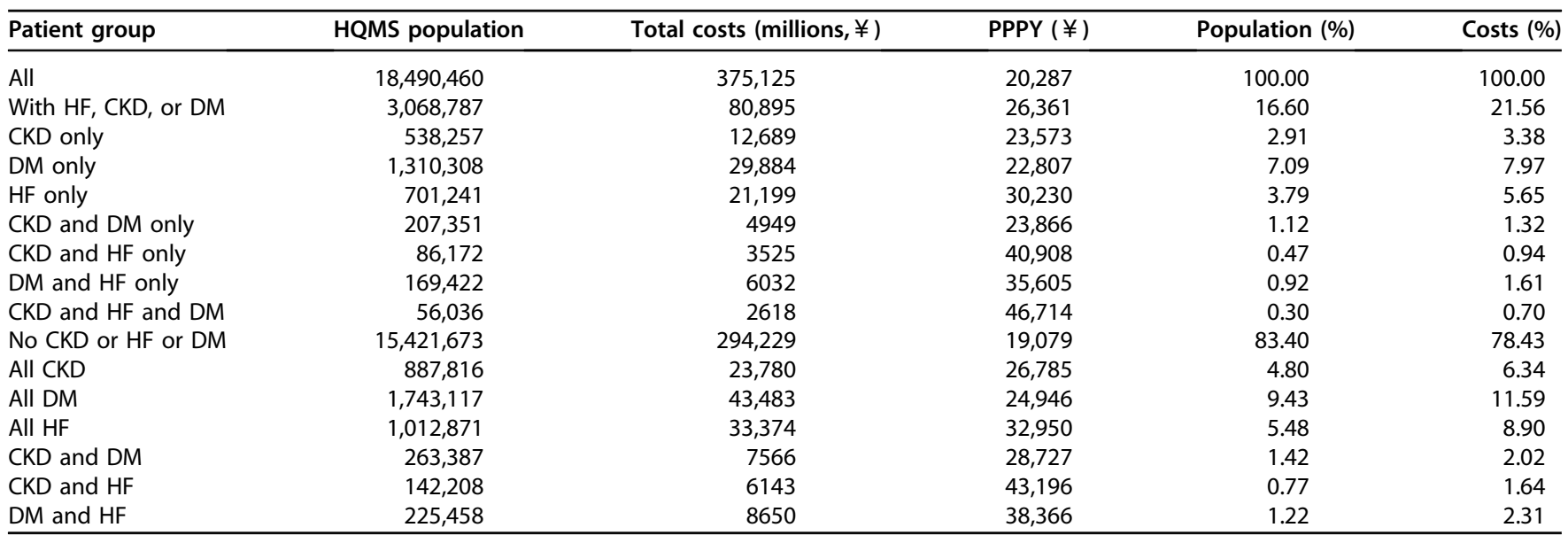

CKD, chronic kidney disease; DM, diabetes mellitus; HF, heart failure; PPPY, per person per year. 


\subsubsection{Costs stratified by types of health insurance.}

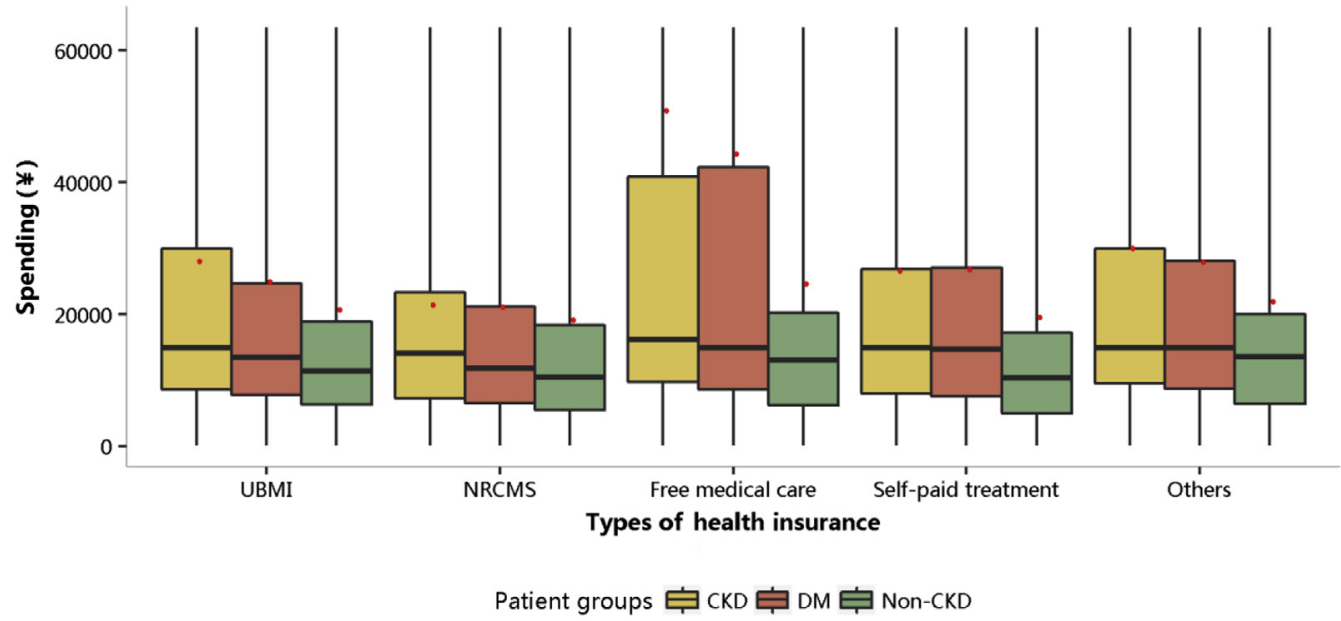

Figure 39| Costs stratified by types of health insurance. Limited to $1.5 *$ third quartile. Red points refer to cost per person per year. CKD, chronic kidney disease; DM, diabetes mellitus; NRCMS, new rural co-operative medical care; UBMI, urban basic medical insurance.

Table 39| Costs stratified by types of health insurance (median [Q1-Q3])

\begin{tabular}{lcrr}
\hline Types of health insurance & CKD & DM & Non-CKD \\
\hline UBMI & $14,965(8559-29,930)$ & $13,482(7810-24,645)$ & $11,365(6289-18,825)$ \\
NRCMS & $14,135(7259-23,348)$ & $11,815(6562-21,204)$ & $10,467(5495-18,344)$ \\
Free medical care & $16,128(9710-40,825)$ & $14,965(8576-42,324)$ & $13,044(6267-20,248)$ \\
Self-paid treatment & $14,965(7998-26,896)$ & $14,676(7587-27,079)$ & $10,343(4929-17,174)$ \\
Others & $14,965(9538-29,930)$ & $14,965(8663-28,136)$ & $13,554(6453-20,023)$ \\
Total & $14,965(8302-28,282)$ & $13,749(7,666-24,955)$ & $1,219(5867-18,556)$ \\
\hline
\end{tabular}

CKD, chronic kidney disease; DM, diabetes mellitus; NRCMS, new rural co-operative medical care; Q1, first quartile; Q3, third quartile; UBMI, urban basic medical insurance. The average cost was used in place of missing costs.

Table $40 \mid$ Costs stratified by types of health insurance (mean [SD])

\begin{tabular}{lllr}
\hline Types of health insurance & \multicolumn{1}{c}{ CKD } & DM & Non-CKD \\
\hline UBMI & $27,692(47,466)$ & $24,549(39,862)$ & $20,274(32,335)$ \\
NRCMS & $21,013(30,695)$ & $20,659(29,700)$ & $18,757(26,997)$ \\
Free medical care & $50,445(118,912)$ & $43,986(97,367)$ & $24,248(52,717)$ \\
Self-paid treatment & $26,195(45,805)$ & $26,411(46,150)$ & $19,122(32,692)$ \\
Others & $29,648(53,271)$ & $27,593(48,123)$ & $21,540(34,362)$ \\
Total & $26,785(47,949)$ & $24,946(42,630)$ & $19,960(32,221)$ \\
\hline
\end{tabular}

CKD, chronic kidney disease; DM, diabetes mellitus; NRCMS, new rural co-operative medical care; UBMI, urban basic medical insurance. The average cost was used in place of missing costs.

\subsubsection{Costs stratified by sex.}

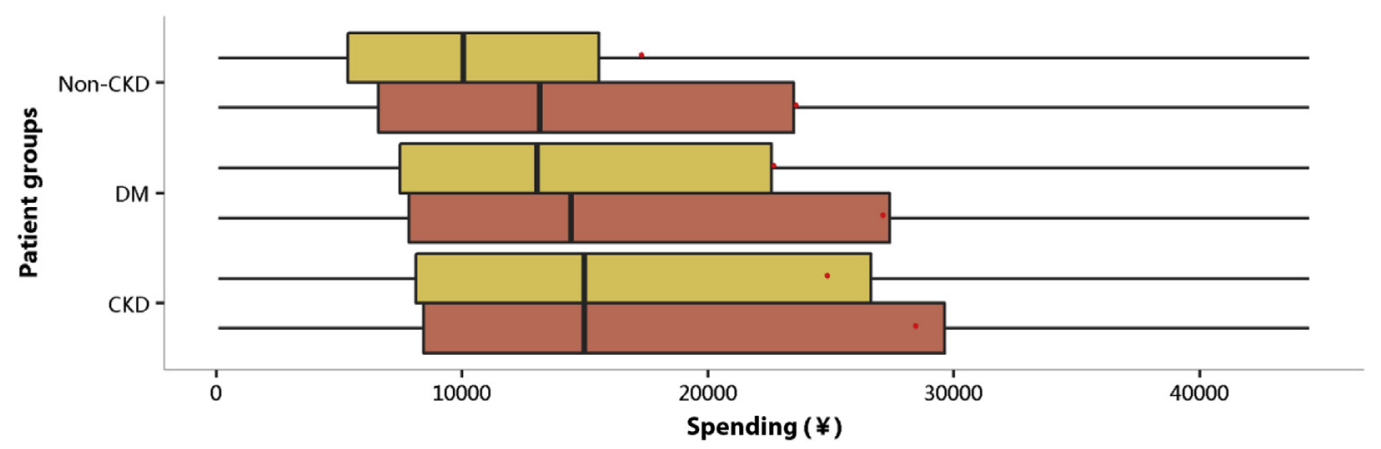

Sex 庐 Male 宁 Female

Figure 40 | Costs stratified by sex. Limited to $1.5 *$ third quartile. Red points refer to cost per person per year. CKD, chronic kidney disease; DM, diabetes mellitus. 
Table 41 | Costs stratified by sex (median [Q1-Q3])

\begin{tabular}{lcrr}
\hline Sex & CKD & DM & Non-CKD \\
\hline Male & $14,965(8447-29,630)$ & $14,430(7842-27,387)$ & $13,161(6600-23,479)$ \\
Female & $14,965(8124-26,608)$ & $13,060(7473-22,588)$ & $10,038(5359-15,578)$ \\
Total & $14,965(8302-28,282)$ & $13,749(7666-24,955)$ & $11,219(5867-18,556)$ \\
\hline
\end{tabular}

CKD, chronic kidney disease; DM, diabetes mellitus; Q1, first quartile; Q3, third quartile.

The average cost was used in place of missing costs.

Table 42 | Costs stratified by sex (mean [SD])

\begin{tabular}{lclr}
\hline Sex & CKD & DM & Non-CKD \\
\hline Male & $28,294(52,798)$ & $26,982(47,979)$ & $23,456(37,713)$ \\
Female & $24,707(40,237)$ & $22,550(35,164)$ & $17,178(26,741)$ \\
Total & $26,785(47,949)$ & $24,946(42,630)$ & $19,960(32,221)$ \\
\hline
\end{tabular}

CKD, chronic kidney disease; DM, diabetes mellitus.

The average cost was used in place of missing costs.

\subsubsection{Costs stratified by age.}

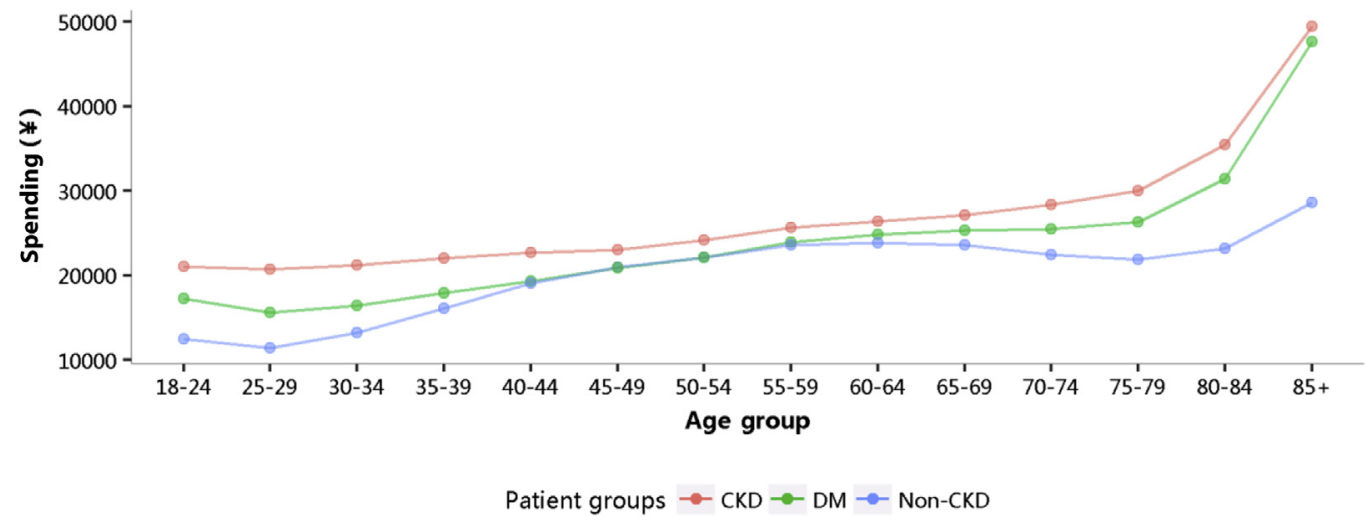

Figure 41 | Costs stratified by age group (mean). CKD, chronic kidney disease; DM, diabetes mellitus.

Table 43 | Costs stratified by age group (median [Q1-Q3])

\begin{tabular}{lcrr}
\hline Age group & CKD & \multicolumn{1}{c}{ DM } & Non-CKD \\
\hline $18-24$ & $12,868(6578-20,570)$ & $8177(5075-14,965)$ & $7232(3677-14,965)$ \\
$25-29$ & $12,458(6412-20,801)$ & $8757(5419-14,965)$ & $7264(3910-14,965)$ \\
$30-34$ & $12,929(6639-21,390)$ & $9072(5706-14,965)$ & $8282(4404-14,965)$ \\
$35-39$ & $13,814(6956-22,578)$ & $9799(6082-15,646)$ & $9585(5068-14,965)$ \\
$40-44$ & $14,438(7317-23,434)$ & $10,736(6431-17,692)$ & $11,144(5706-17,720)$ \\
$45-49$ & $14,965(7581-24,555)$ & $11,552(6747-19,682)$ & $12,080(6096-20,120)$ \\
$50-54$ & $14,965(8010-26,071)$ & $12,204(7069-21,171)$ & $12,423(6367-21,626)$ \\
$55-59$ & $14,965(8472-27,893)$ & $13,067(7473-23,673)$ & $13,103(6743-24,145)$ \\
$60-64$ & $14,965(8737-29,071)$ & $13,759(7771-25,423)$ & $13,442(6931-24,899)$ \\
$65-69$ & $14,965(8878-29,930)$ & $14,414(8019-26,619)$ & $13,583(7050-24,656)$ \\
$70-74$ & $14,965(9094-30,094)$ & $14,965(8287-27,241)$ & $13,523(7115-23,307)$ \\
$75-79$ & $15,428(9438-31,993)$ & $14,965(8626-28,680)$ & $13,657(7235-22,654)$ \\
$80-84$ & $16,966(9958-36,284)$ & $14,965(9218-30,836)$ & $14,085(7337-23,451)$ \\
$85+$ & $19,232(10,708-44,895)$ & $17,755(10,311-41,595)$ & $14,965(7513-26,767)$ \\
Total & $14,965(8302-28,282)$ & $13,749(7666-24,955)$ & $11,219(5867-18,556)$ \\
\hline
\end{tabular}

CKD, chronic kidney disease; DM, diabetes mellitus; Q1, first quartile; Q3, third quartile.

The average cost was used in place of missing costs. 
Table 44| Costs stratified by age group (mean [SD])

\begin{tabular}{lllr}
\hline Age group & CKD & \multicolumn{1}{c}{ DM } & Non-CKD \\
\hline $18-24$ & $21,093(81,100)$ & $17,241(129,772)$ & $12,522(23,974)$ \\
$25-29$ & $20,741(36,115)$ & $15,651(32,672)$ & $11,479(20,056)$ \\
$30-34$ & $21,268(37,763)$ & $16,465(31,968)$ & $13,219(22,777)$ \\
$35-39$ & $22,057(36,853)$ & $17,926(33,302)$ & $16,143(27,248)$ \\
$40-44$ & $22,694(38,247)$ & $19,309(32,710)$ & $19,112(30,383)$ \\
$45-49$ & $23,010(36,132)$ & $20,930(33,937)$ & $20,982(31,997)$ \\
$50-54$ & $24,225(37,259)$ & $22,102(34,230)$ & $22,126(33,065)$ \\
$55-59$ & $25,691(38,587)$ & $23,942(36,606)$ & $23,604(34,306)$ \\
$60-64$ & $26,385(38,710)$ & $24,879(36,165)$ & $23,856(33,718)$ \\
$65-69$ & $27,191(40,578)$ & $25,335(37,101)$ & $23,576(33,207)$ \\
$70-74$ & $28,348(41,358)$ & $25,504(36,337)$ & $22,475(31,598)$ \\
$75-79$ & $30,024(46,850)$ & $26,336(39,636)$ & $21,902(32,156)$ \\
$80-84$ & $35,452(66,825)$ & $31,458(61,222)$ & $23,176(42,685)$ \\
$85+$ & $49,420(109,236)$ & $47,668(108,242)$ & $28,625(68,435)$ \\
Total & $26,785(47,949)$ & $24,946(42,630)$ & $19,960(32,221)$ \\
\hline
\end{tabular}

CKD, chronic kidney disease; DM, diabetes mellitus.

The average cost was used in place of missing costs.

\subsection{Length of stay}

\subsubsection{Overall length of stay stratified by CKD, DM, and heart failure.}

Table 45 | Overall length of stay stratified by CKD, DM, and heart failure

\begin{tabular}{|c|c|c|c|c|c|}
\hline Patient group & HQMS population & Total LOS (thousands, days) & PPPY (days) & Population (\%) & LOS (\%) \\
\hline All & $18,490,460$ & 257,250 & 13.91 & 100.00 & 100.00 \\
\hline With HF, CKD, or DM & $3,068,787$ & 55,482 & 18.08 & 16.64 & 21.58 \\
\hline CKD only & 538,257 & 9923 & 18.44 & 2.91 & 3.86 \\
\hline HF only & 701,241 & 11,997 & 17.11 & 3.79 & 4.66 \\
\hline CKD and DM only & 207,351 & 4182 & 20.17 & 1.12 & 1.62 \\
\hline CKD and HF only & 86,172 & 2542 & 29.49 & 0.47 & 0.99 \\
\hline No CKD or HF or DM & $15,421,673$ & 201,768 & 13.08 & 83.36 & 78.42 \\
\hline All CKD & 887,816 & 18,521 & 20.86 & 4.81 & 7.21 \\
\hline All DM & $1,743,117$ & 31,020 & 17.80 & 9.43 & 12.06 \\
\hline All HF & $1,012,871$ & 19,896 & 19.64 & 5.48 & 7.73 \\
\hline CKD and DM & 263,387 & 6056 & 22.99 & 1.43 & 2.35 \\
\hline CKD and $\mathrm{HF}$ & 142,208 & 4415 & 31.05 & 0.77 & 1.72 \\
\hline $\mathrm{DM}$ and $\mathrm{HF}$ & 225,458 & 5357 & 23.76 & 1.22 & 2.08 \\
\hline
\end{tabular}

CKD, chronic kidney disease; DM, diabetes mellitus; HF, heart failure; LOS, length-of-stay; PPPY, per person per year.

\subsubsection{Length of stay stratified by types of health insurance.}

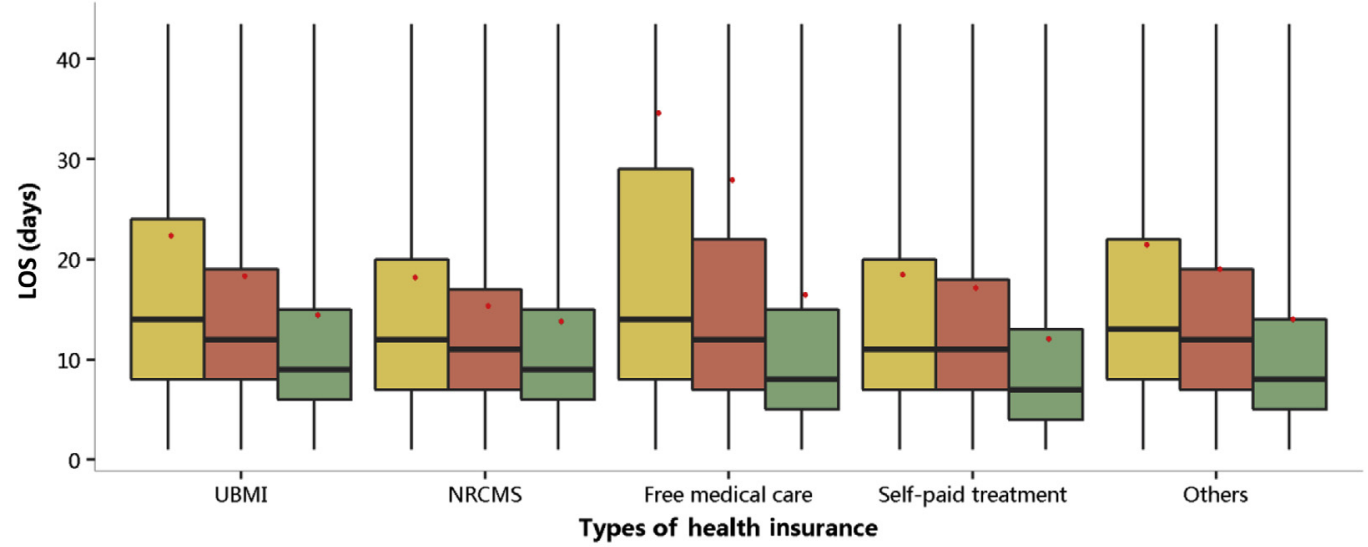

Patient groups 宁 CKD 宁 DM 宁 Non-CKD

Figure 42 | Length of stay stratified by types of health insurance. CKD, chronic kidney disease; DM, diabetes mellitus; NRCMS, new rural cooperative medical care; UBMI, urban basic medical insurance. Limited to $1.5^{*}$ third quartile. Red points refer to length of stay per person per year. 
Table 46 | Length of stay stratified by types of health insurance (median [Q1-Q3])

\begin{tabular}{llr}
\hline Types of health insurance & CKD & DM \\
\hline UBMI & $2(8-24)$ & $12(8-19)$ \\
NRCMS & $12(7-20)$ & $11(7-17)$ \\
Free medical care & $14(8-29)$ & $12(7-22)$ \\
Self-paid treatment & $11(7-20)$ & $11(7-18)$ \\
Others & $13(8-22)$ & $12(7-19)$ \\
Total & $13(8-22)$ & $7(5-15)$ \\
\hline
\end{tabular}

The missing length of stay was filled with average length of stay.

CKD, chronic kidney disease; DM, diabetes mellitus; NRCMS, new rural co-operative medical care; Q1, first quartile; Q3, third quartile; UBMI, urban basic medical insurance.

Table 47| Length of stay stratified by types of health insurance (mean [SD])

\begin{tabular}{lcrr}
\hline Types of health insurance & CKD & DM & Non-CKD \\
\hline UBMI & $22.10(36.53)$ & $18.09(27.63)$ & $14.18(21.72)$ \\
NRCMS & $17.96(26.75)$ & $15.06(18.24)$ & $13.59(17.61)$ \\
Free medical care & $34.33(65.23)$ & $27.67(56.68)$ & $16.23(35.30)$ \\
Self-paid treatment & $18.25(29.73)$ & $16.91(25.85)$ & $11.81(18.79)$ \\
Others & $21.25(35.42)$ & $18.76(30.32)$ & $13.76(22.15)$ \\
Total & $20.86(34.68)$ & $17.80(27.66)$ & $13.56(20.81)$ \\
\hline
\end{tabular}

CKD, chronic kidney disease; DM, diabetes mellitus; NRCMS, new rural co-operative medical care; UBMI, urban basic medical insurance.

The average length of stay was used in place of missing lengths of stay.

\subsubsection{Length of stay stratified by sex.}

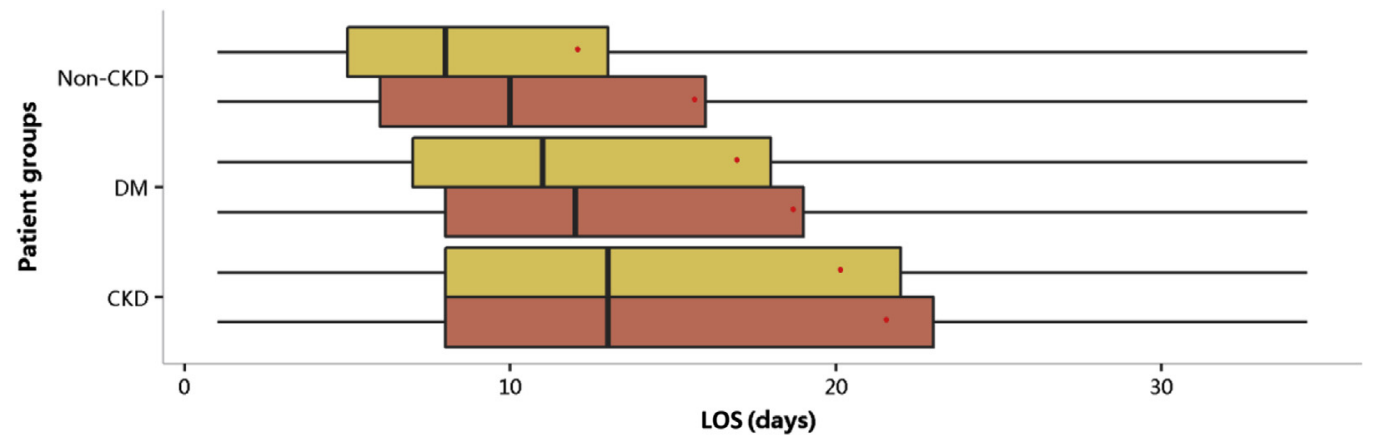

Sex 审 Male 官 Female

Figure 43 | Length of stay stratified by sex. Limited to $1.5^{*}$ third quartile. Red points refer to length of stay per person per year. CKD, chronic kidney disease; DM, diabetes mellitus.

Table 48 | Length of stay stratified by sex (median [Q1-Q3])

\begin{tabular}{lccr}
\hline Sex & CKD & DM & Non-CKD \\
\hline Male & $13(8-23)$ & $12(8-19)$ & $10(6-16)$ \\
Female & $13(8-22)$ & $11(7-18)$ & $8(5-13)$ \\
Total & $13(8-22)$ & $11(7-19)$ & $8(5-14)$ \\
\hline
\end{tabular}

CKD, chronic kidney disease; DM, diabetes mellitus; Q1, first quartile; Q3, third quartile.

The average length of stay was used in place of missing lengths of stay.

Table 49 | Length of stay stratified by sex (mean [SD])

\begin{tabular}{|c|c|c|c|}
\hline Sex & CKD & DM & Non-CKD \\
\hline Male & $21.45(36.64)$ & $18.60(30.31)$ & $15.56(23.87)$ \\
\hline Female & 20.05 (31.76) & $16.85(24.13)$ & 11.97 (17.85) \\
\hline Total & $20.86(34.68)$ & $17.80(27.66)$ & $13.56(20.81)$ \\
\hline
\end{tabular}

CKD, chronic kidney disease; DM, diabetes mellitus.

The average length of stay was used in place of missing lengths of stay. 


\subsubsection{Length of stay stratified by age.}

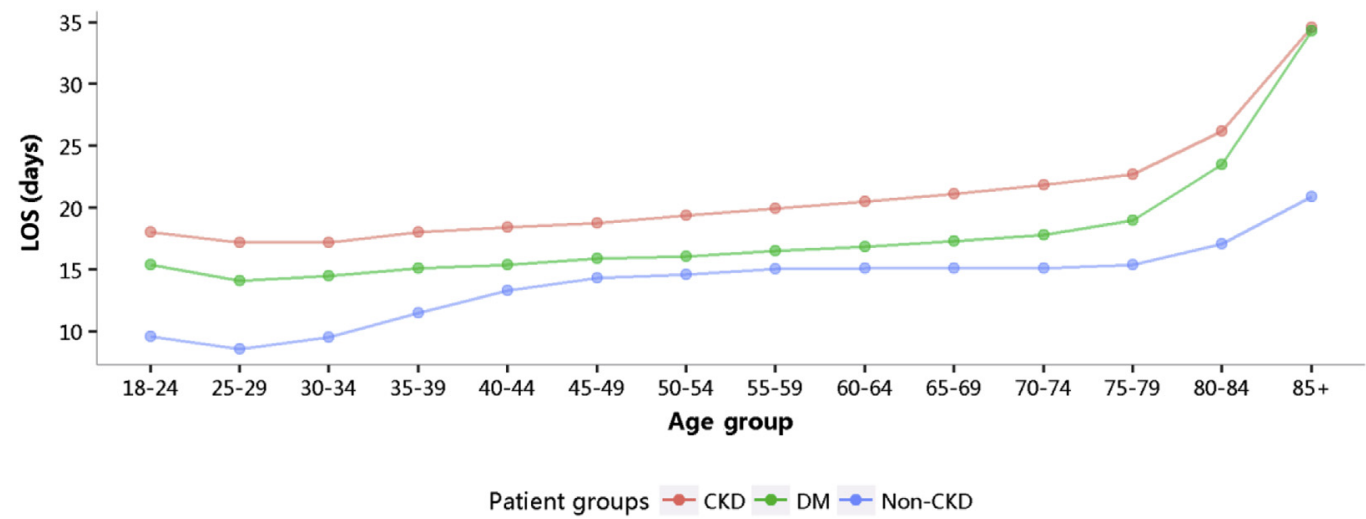

Figure 44 | Length of stay stratified by age group (mean). CKD, chronic kidney disease; DM, diabetes mellitus.

Table 50| Length of stay stratified by age group (median [Q1-Q3])

\begin{tabular}{lccr}
\hline Age group & CKD & DM & Non-CKD \\
\hline $18-24$ & $12(7-20)$ & $10(7-15)$ & $6(4-10)$ \\
$25-29$ & $11(6-19)$ & $10(7-15)$ & $6(4-9)$ \\
$30-34$ & $11(6-18)$ & $10(7-15)$ & $6(4-10)$ \\
$35-39$ & $11(7-19)$ & $10(7-15)$ & $7(4-12)$ \\
$40-44$ & $12(7-20)$ & $11(7-16)$ & $8(5-14)$ \\
$45-49$ & $12(7-20)$ & $11(7-16)$ & $9(6-15)$ \\
$50-54$ & $12(8-21)$ & $11(7-17)$ & $9(6-15)$ \\
$55-59$ & $13(8-22)$ & $11(7-17)$ & $9(6-16)$ \\
$60-64$ & $13(8-22)$ & $11(7-18)$ & $10(6-16)$ \\
$65-69$ & $13(8-23)$ & $12(8-19)$ & $10(6-17)$ \\
$70-74$ & $14(9-24)$ & $12(8-20)$ & $10(6-17)$ \\
$75-79$ & $14(9-26)$ & $12(8-21)$ & $13(8-23)$ \\
$80-84$ & $15(9-28)$ & $15(9-29)$ & $11(7-18)$ \\
$85+$ & $16(9-31)$ & $11(7-19)$ & $11(7-19)$ \\
Total & $13(8-22)$ & & $8(5-14)$ \\
\hline
\end{tabular}

CKD, chronic kidney disease; DM, diabetes mellitus; Q1, first quartile; Q3, third quartile.

The average length of stay was used in place of missing lengths of stay.

Table 51 | Length of stay stratified by age group (mean [SD])

\begin{tabular}{lcrr}
\hline Age group & CKD & DM & Non-CKD \\
\hline $18-24$ & $18.05(72.74)$ & $15.38(110.83)$ & $9.59(16.34)$ \\
$25-29$ & $17.20(30.51)$ & $14.10(18.83)$ & $8.62(14.18)$ \\
$30-34$ & $17.21(29.28)$ & $14.49(20.79)$ & $9.57(15.70)$ \\
$35-39$ & $18.01(32.80)$ & $15.11(26.17)$ & $11.50(18.90)$ \\
$40-44$ & $18.42(29.57)$ & $15.38(20.90)$ & $13.34(20.72)$ \\
$45-49$ & $18.75(30.17)$ & $16.07(23.68)$ & $14.30(21.69)$ \\
$50-54$ & $19.36(29.43)$ & $16.55(22.87)$ & $14.61(21.29)$ \\
$55-59$ & $19.93(28.70)$ & $16.89(22.07)$ & $15.06(21.45)$ \\
$60-64$ & $20.54(28.71)$ & $17.29(22.34)$ & $15.12(20.38)$ \\
$65-69$ & $21.15(29.32)$ & $17.84(23.77)$ & $15.13(19.68)$ \\
$70-74$ & $21.89(30.55)$ & $23.99(26.64)$ & $15.11(19.74)$ \\
$75-79$ & $22.68(31.21)$ & $34.29(41.52)$ & $15.37(21.29)$ \\
$80-84$ & $26.22(42.55)$ & $17.80(27.66)$ & $17.06(29.45)$ \\
$85+$ & $34.56(63.77)$ & & $20.94(42.60)$ \\
Total & $20.86(34.68)$ & & $13.56(20.81)$ \\
\hline
\end{tabular}

CKD, chronic kidney disease; DM, diabetes mellitus.

The average length of stay was used in place of missing lengths of stay. 


\title{
Chapter 4: In-hospital mortality of hospitalized patients with CKD
}

\author{
Bixia Gao ${ }^{1}$, Jianyan Long ${ }^{2}$, Chu Hong ${ }^{1}$, Fang Wang ${ }^{1}$, Lanxia Gan ${ }^{3}$ and Haibo Wang ${ }^{3,4}$ \\ ${ }^{1}$ Renal Division, Department of Medicine, Peking University First Hospital; Peking University Institute of Nephrology, Beijing, China; \\ ${ }^{2}$ Clinical Trial Unit, The First Affiliated Hospital of Sun Yat-sen University, Guangzhou, China; ${ }^{3}$ China Standard Medical Infor- \\ mation Research Center, Shenzhen, China; and ${ }^{4}$ Center for Data Science in Health and Medicine, Peking University, Beijing, China
}

This chapter focuses on the in-hospital mortality of inpatients with CKD. Stratified analyses based on comorbidity, types of insurance, age and sex are also provided.

In 2015, the in-hospital mortality rate of inpatients with CKD was $2.63 \%$, which was higher than that of those without CKD $(0.81 \%)$ and with diabetes mellitus (DM, 1.54\%) (Tables 52 and 53). This trend was consistent across age-sex subgroups and across different types of medical insurance (Figures 45-47 and Tables 53-55). The in-hospital mortality rate of inpatients with CKD increased with age (Figure 47 and Table 55). Moreover, male inpatients with CKD had a higher in-hospital mortality rate than did female inpatients $(2.80 \%$ vs. 2.39\%) (Figure 46 and Table 54). Among the hospitalized patients with CKD, those with heart failure had a remarkably higher rate of in-hospital mortality (7.84\%) (Table 52).

Among different types of insurance, hospitalized CKD patients with free medical care had the highest in-hospital mortality rate $(5.54 \%)$, followed by those with urban basic medical insurance (UBMI, 3.48\%) (Figure 45 and Table 53), which could partly be explained by the high percentage of diabetic kidney disease DKD and hypertensive nephropathy HTN among urban residents.

\subsection{In-hospital mortality rate stratified by CKD, DM, and heart failure}

Table 52 | In-hospital mortality rate stratified by CKD, DM, and heart failure

\begin{tabular}{|c|c|c|c|c|}
\hline Patient group & Hospital mortality & HQMS population & Mortality rate (\%) & Proportion (\%) \\
\hline All & 166,310 & $18,490,460$ & 0.90 & 100.00 \\
\hline With HF or CKD or DM & 72,683 & $3,068,787$ & 2.37 & 43.70 \\
\hline CKD only & 8704 & 538,257 & 1.62 & 5.23 \\
\hline HF only & 29,933 & 701,241 & 4.27 & 18.00 \\
\hline CKD and DM only & 3468 & 207,351 & 1.67 & 2.09 \\
\hline CKD and HF only & 7197 & 86,172 & 8.35 & 4.33 \\
\hline No CKD or HF or DM & 93,627 & $15,421,673$ & 0.61 & 56.30 \\
\hline All CKD & 23,316 & 887,816 & 2.63 & 14.02 \\
\hline All DM & 26,849 & $1,743,117$ & 1.54 & 16.14 \\
\hline All HF & 47,655 & $1,012,871$ & 4.70 & 28.65 \\
\hline CKD and DM & 7415 & 263,387 & 2.82 & 4.46 \\
\hline CKD and HF & 11,144 & 142,208 & 7.84 & 6.70 \\
\hline
\end{tabular}

CKD, chronic kidney disease; DM, diabetes mellitus; HF, heart failure; HQMS, Hospital Quality Monitoring System. 


\subsection{In-hospital mortality rate stratified by types of insurance}

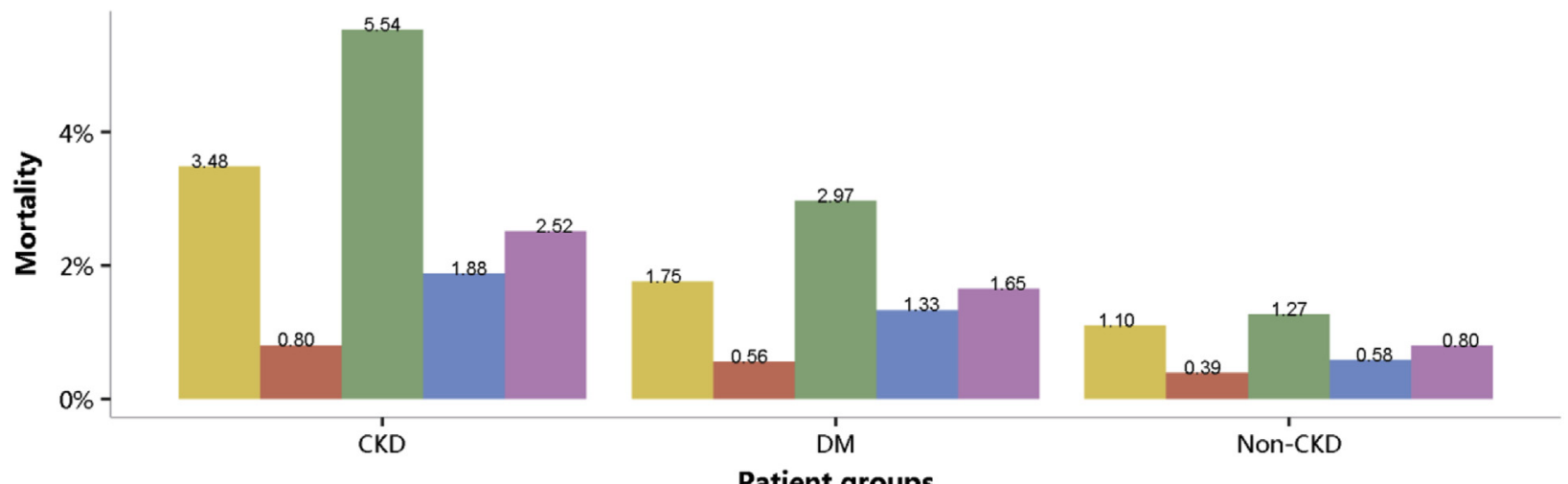

Types of Health Insurance | UBMI| NRCMS | Free medical care| Self-paid treatment |Others

Figure 45 | In-hospital mortality rate stratified by different types of insurance. CKD, chronic kidney disease; DM, diabetes mellitus; NRCMS, new rural co-operative medical care; UBMI, urban basic medical insurance.

Table 53 | In-hospital mortality rate stratified by different types of insurance, $N$ (\%)

\begin{tabular}{lrrr}
\hline Types of health insurance & \multicolumn{1}{c}{ CKD } & DM & Non-CKD \\
\hline UBMI & $15,850(3.48)$ & $18,433(1.75)$ & $86,157(1.10)$ \\
NRCMS & $1,525(0.80)$ & $1493(0.56)$ & $14,260(0.39)$ \\
Free medical care & $1067(5.54)$ & $1216(2.97)$ & $4585(1.27)$ \\
Self-paid treatment & $2256(1.88)$ & $2553(1.33)$ & $20,866(0.58)$ \\
Others & $2618(2.52)$ & $3154(1.65)$ & $17,126(0.80)$ \\
Total & $23,316(2.63)$ & $26,849(1.54)$ & $142,994(0.81)$ \\
\hline
\end{tabular}

CKD, chronic kidney disease; DM, diabetes mellitus; NRCMS: new rural co-operative medical care; UBMI, urban basic medical insurance.

\subsection{In-hospital mortality rate stratified by sex}

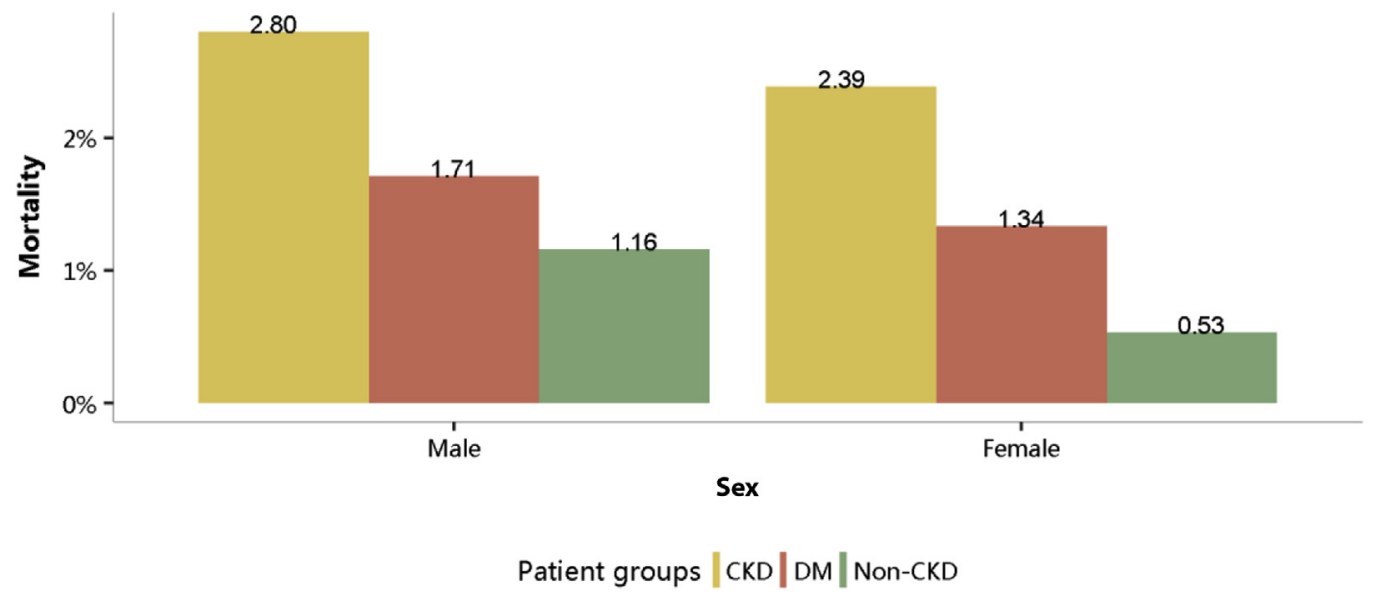

Figure 46 | In-hospital mortality rate stratified by sex. CKD, chronic kidney disease; DM, diabetes mellitus.

Table 54 | In-hospital mortality rate stratified by sex, $N$ (\%)

\begin{tabular}{lcrr}
\hline Patient group & Male & \multicolumn{1}{c}{ Female } & Total \\
\hline CKD & $14,405(2.80)$ & $8911(2.39)$ & $23,316(2.63)$ \\
DM & $16,144(1.71)$ & $10,705(1.34)$ & $26,849(1.54)$ \\
Non-CKD & $90,680(1.16)$ & $52,314(0.53)$ & $142,994(0.81)$ \\
\hline
\end{tabular}

CKD, chronic kidney disease; DM, diabetes mellitus. 


\subsection{In-hospital mortality rate stratified by age}

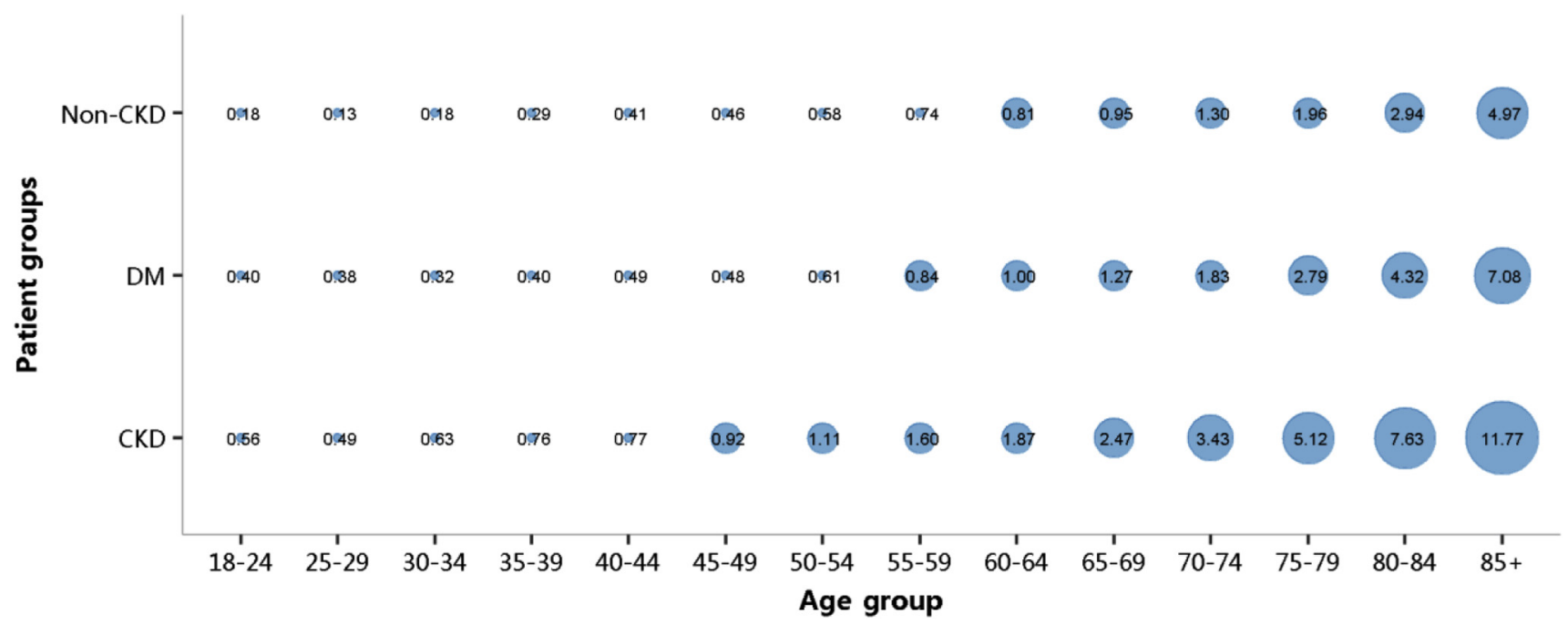

Figure 47| In-hospital mortality rate stratified by age group. Point size refers to mortality rate. CKD, chronic kidney disease; DM diabetes mellitus.

Table 55 | In-hospital mortality rate stratified by age group, $N$ (\%)

\begin{tabular}{lrrr}
\hline Age group & \multicolumn{1}{c}{ CKD } & DM & Non-CKD \\
\hline $18-24$ & $136(0.56)$ & $27(0.40)$ & $1730(0.18)$ \\
$25-29$ & $166(0.49)$ & $46(0.38)$ & $2092(0.13)$ \\
$30-34$ & $222(0.63)$ & $63(0.32)$ & $2187(0.18)$ \\
$35-39$ & $315(0.76)$ & $131(0.40)$ & $3090(0.29)$ \\
$40-44$ & $470(0.77)$ & $334(0.49)$ & $5311(0.41)$ \\
$45-49$ & $724(0.92)$ & $1189(0.48)$ & $7286(0.46)$ \\
$50-54$ & $1017(1.11)$ & $1850(0.84)$ & $9838(0.58)$ \\
$55-59$ & $1396(1.60)$ & $2765(1.00)$ & $12,135(0.74)$ \\
$60-64$ & $1910(1.87)$ & $3217(1.27)$ & $15,119(0.81)$ \\
$65-69$ & $2235(2.47)$ & $3878(1.83)$ & $14,520(0.95)$ \\
$70-74$ & $2666(3.43)$ & $4879(2.79)$ & $15,689(1.30)$ \\
$75-79$ & $3866(5.12)$ & $4575(4.32)$ & $19,706(1.96)$ \\
$80-84$ & $4176(7.63)$ & $3329(7.08)$ & $18,486(2.94)$ \\
$85+$ & $4017(11.77)$ & $26,849(1.54)$ & $15,805(4.97)$ \\
Total & $23,316(2.63)$ & $142,994(0.81)$ \\
\hline
\end{tabular}

CKD, chronic kidney disease; DM, diabetes mellitus. 


\title{
Chapter 5: Acute kidney injury
}

\author{
Chao Yang ${ }^{1}$, Jianyan Long ${ }^{2}$, Zaiming $\mathrm{Su}^{3}$, Rui $\mathrm{Chen}^{1}$, Lili Liu ${ }^{1}$, Fang Wang ${ }^{1}$ and Haibo Wang ${ }^{3,4}$
}

${ }^{1}$ Renal Division, Department of Medicine, Peking University First Hospital; Peking University Institute of Nephrology, Beijing, China; ${ }^{2}$ Clinical Trial Unit, The First Affiliated Hospital of Sun Yat-sen University, Guangzhou, China; ${ }^{3}$ Center for Data Science in Health and Medicine, Peking University, Beijing, China; and ${ }^{4}$ China Standard Medical Information Research Center, Shenzhen, China

AKI is a major risk factor for the development of CKD. This chapter focuses on inpatients with AKI. Of note, as AKI is often underdiagnosed, results reflect both the disease burden and the diagnostic rate.

The percentage of patients with the diagnostic coding of AKI was $0.30 \%$, with a male predominance $(64.84 \%)$ (Figure 48 and Table 56). Inpatients aged 60 to 64,65 to 69 , and 75 to 79 years were more likely to be diagnosed with AKI (Figure 49 and Table 57). Compared with inpatients without an intensive care unit (ICU) stay, the percentage of AKI among inpatients with ICU stay was significantly higher $(3.93 \%$ vs. $0.25 \%)$ (Table 58$)$. There were substantial geographic variations regarding the percentage of AKI among patients with an ICU stay (Figure 50).

Altogether $1.69 \%$ of patients with CKD were diagnosed with AKI. Regarding the causes of CKD, patients with CTIN and GN had relatively high percentages of diagnosed AKI (3.63\% and 2.81\%, respectively) (Figure 51 and Table 59). Among inpatients with AKI, 27.13\% had diagnoses of CKD, and $16.67 \%$ had diabetes (Tables 60 and 61). The percentage of CKD decreased with age and was higher among female patients (Figure 52), which might partly reflect survivorship bias. Male patients aged 60 to 64 years and female patients aged 65 to 69 years had the highest percentage of diabetes, respectively (Figure 53).

\subsection{Characteristics of AKI}

\subsubsection{Sex distribution of AKI, stratified by age.}

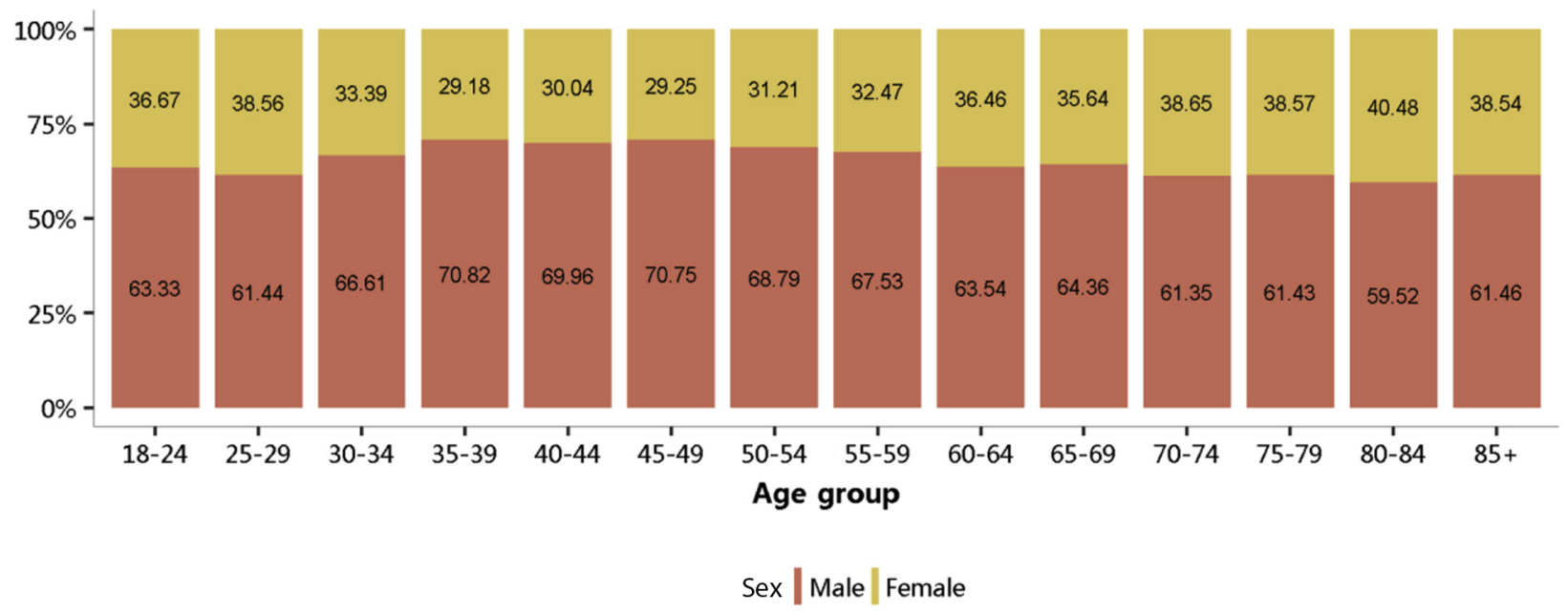

Figure 48 | Sex distribution of AKI, stratified by age. AKI, acute kidney injury. 
Table 56| Sex distribution of AKI, stratified by age, $N$ (\%)

\begin{tabular}{|c|c|c|c|}
\hline Age group & Male & Female & Total \\
\hline $18-24$ & 1069 (63.33) & $619(36.67)$ & 1688 \\
\hline $25-29$ & $1211(61.44)$ & $760(38.56)$ & 1971 \\
\hline $30-34$ & $1195(66.61)$ & 599 (33.39) & 1794 \\
\hline $35-39$ & $1461(70.82)$ & 602 (29.18) & 2063 \\
\hline $40-44$ & 2315 (69.96) & 994 (30.04) & 3309 \\
\hline $45-49$ & 3072 (70.75) & $1270(29.25)$ & 4342 \\
\hline $50-54$ & 3394 (68.79) & $1540(31.21)$ & 4934 \\
\hline $55-59$ & 3435 (67.53) & 1652 (32.47) & 5087 \\
\hline $60-64$ & 3969 (63.54) & 2277 (36.46) & 6246 \\
\hline $65-69$ & 3601 (64.36) & $1994(35.64)$ & 5595 \\
\hline $70-74$ & 3227 (61.35) & 2033 (38.65) & 5260 \\
\hline $75-79$ & $3453(61.43)$ & 2168 (38.57) & 5621 \\
\hline $80-84$ & 2627 (59.52) & $1787(40.48)$ & 4414 \\
\hline $85+$ & 1890 (61.46) & 1185 (38.54) & 3075 \\
\hline Total & $35,919(64.84)$ & 19,480 (35.16) & 55,399 \\
\hline
\end{tabular}

AKI, acute kidney injury.

\subsubsection{Age distribution of AKI, stratified by sex.}

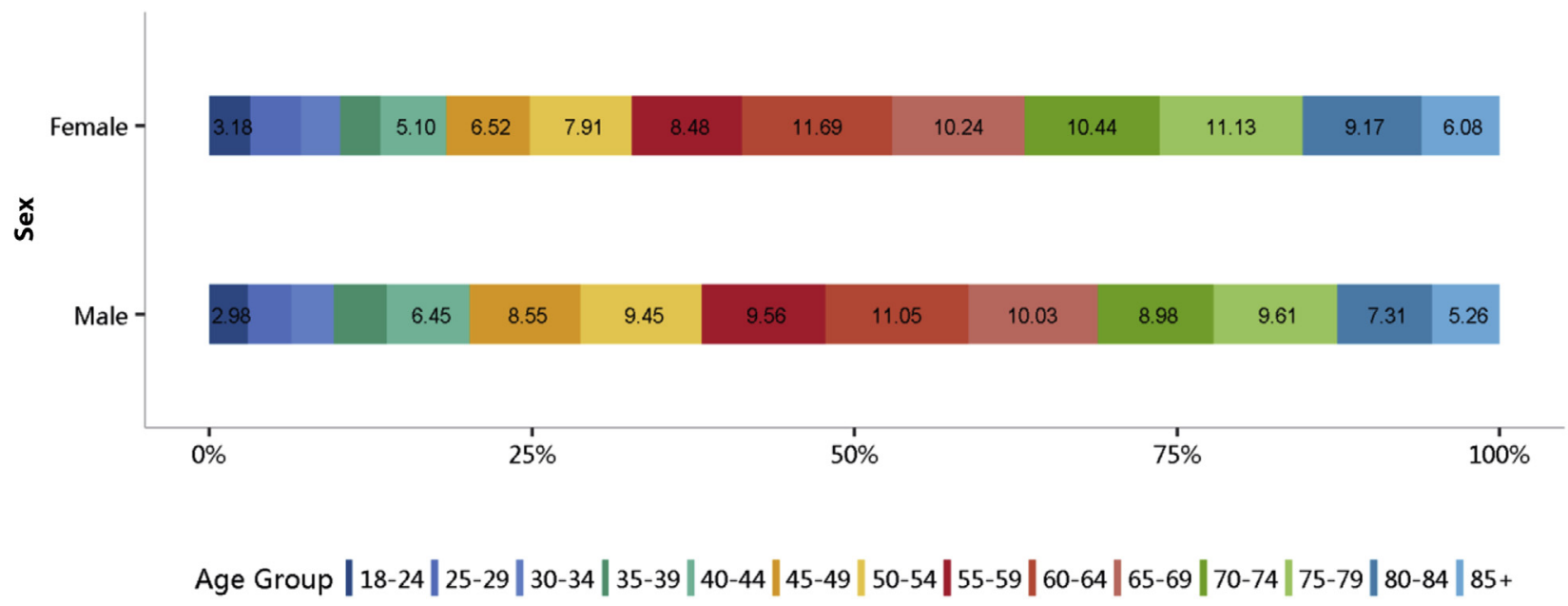

Figure 49 | Age distribution of AKI, stratified by sex. AKI, acute kidney injury.

Table 57| Age distribution of AKI, stratified by sex, $N$ (\%)

\begin{tabular}{|c|c|c|c|}
\hline Age group & Male & Female & Total \\
\hline $18-24$ & 1069 (2.98) & $619(3.18)$ & $1688(3.05)$ \\
\hline $25-29$ & 1211 (3.37) & $760(3.90)$ & $1971(3.56)$ \\
\hline $30-34$ & 1195 (3.33) & 599 (3.07) & 1794 (3.24) \\
\hline $35-39$ & 1461 (4.07) & 602 (3.09) & 2063 (3.72) \\
\hline $40-44$ & $2315(6.45)$ & $994(5.10)$ & 3309 (5.97) \\
\hline $45-49$ & 3072 (8.55) & $1270(6.52)$ & $4342(7.84)$ \\
\hline $50-54$ & 3394 (9.45) & 1540 (7.91) & 4934 (8.91) \\
\hline $55-59$ & 3435 (9.56) & 1652 (8.48) & 5087 (9.18) \\
\hline $60-64$ & 3969 (11.05) & 2277 (11.69) & $6246(11.27)$ \\
\hline $65-69$ & 3601 (10.03) & $1994(10.24)$ & 5595 (10.10) \\
\hline $70-74$ & 3227 (8.98) & $2033(10.44)$ & $5260(9.49)$ \\
\hline $75-79$ & $3453(9.61)$ & 2168 (11.13) & $5621(10.15)$ \\
\hline $80-84$ & $2627(7.31)$ & 1787 (9.17) & 4414 (7.97) \\
\hline $85+$ & $1890(5.26)$ & 1185 (6.08) & 3075 (5.55) \\
\hline Total & 35,919 & 19,480 & 55,399 \\
\hline
\end{tabular}

AKI, acute kidney injury. 


\subsection{Percentage of AKI}

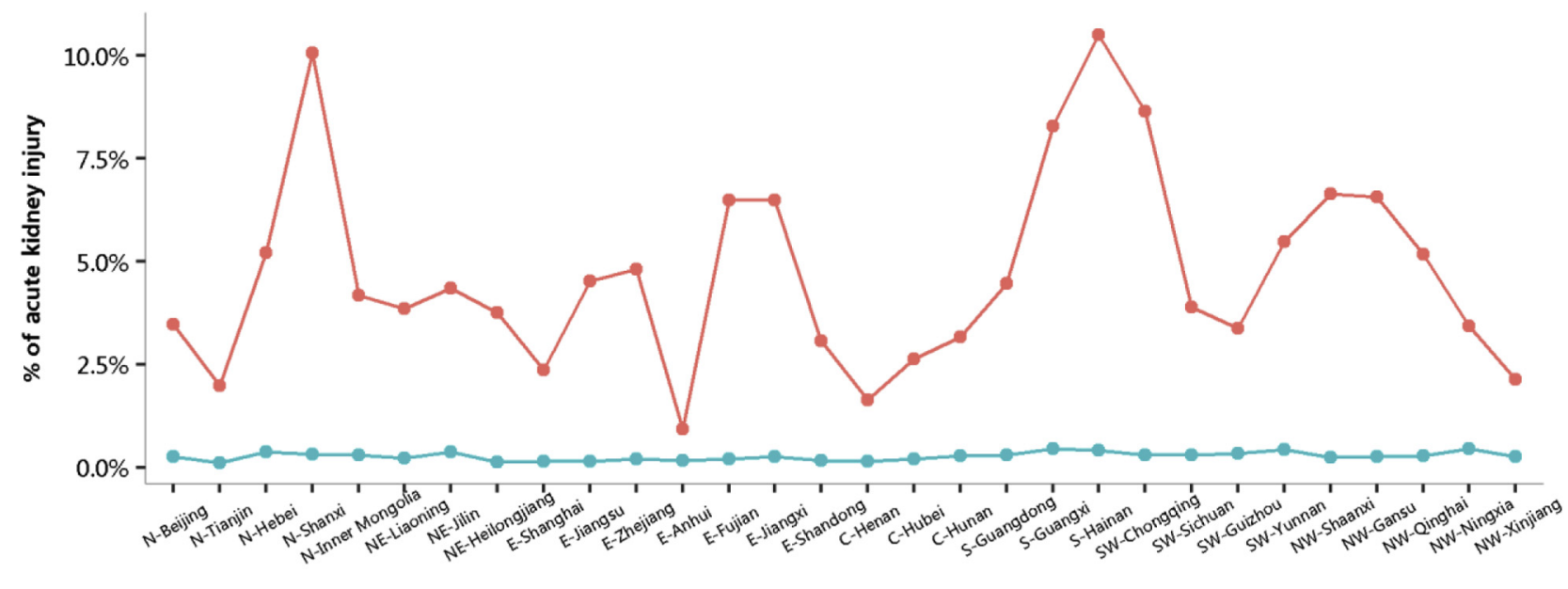

Region

Intensive Care - - with ICU stay $\rightarrow$ - without ICU stay

Figure 50| Percentage of AKI with and without ICU stay, stratified by geographic regions. AKI, acute kidney injury; C, Central of China; $E$, East of China; ICU, intensive care unit; N, North of China; NE, Northeast of China; NW, Northwest of China; S, South of China; SW, Southwest of China.

Table 58 | Percentage of AKI with and without ICU stay, stratified by geographic regions, $N$ (\%)

\begin{tabular}{|c|c|c|c|}
\hline Region & With ICU stay & Without ICU stay & Total \\
\hline N-Beijing & $1190(3.46)$ & $1974(0.26)$ & $3164(0.40)$ \\
\hline N-Tianjin & $29(1.99)$ & $238(0.12)$ & $267(0.14)$ \\
\hline N-Hebei & $229(5.22)$ & $1835(0.37)$ & $2064(0.42)$ \\
\hline N-Inner Mongolia & 77 (4.19) & $1308(0.30)$ & $1385(0.32)$ \\
\hline NE-Liaoning & $280(3.86)$ & $1352(0.23)$ & $1632(0.28)$ \\
\hline NE-Jilin & $76(4.35)$ & $1500(0.39)$ & $1576(0.40)$ \\
\hline E-Jiangsu & $767(4.53)$ & $2334(0.16)$ & $3101(0.21)$ \\
\hline E-Zhejiang & $526(4.80)$ & $1742(0.21)$ & $2268(0.27)$ \\
\hline E-Anhui & $127(0.93)$ & $1004(0.17)$ & $1131(0.18)$ \\
\hline E-Fujian & $430(6.49)$ & $1105(0.20)$ & $1535(0.27)$ \\
\hline E-Jiangxi & $304(6.48)$ & $1583(0.26)$ & $1887(0.31)$ \\
\hline E-Shandong & $312(3.07)$ & $1364(0.17)$ & $1676(0.20)$ \\
\hline S-Hainan & $180(10.51)$ & $772(0.41)$ & $952(0.51)$ \\
\hline SW-Chongqing & $157(8.65)$ & $791(0.31)$ & $948(0.37)$ \\
\hline SW-Sichuan & $695(3.88)$ & $3486(0.31)$ & $4181(0.36)$ \\
\hline SW-Guizhou & $53(3.38)$ & $760(0.34)$ & $813(0.36)$ \\
\hline SW-Yunnan & $433(5.48)$ & $3234(0.44)$ & 3667 (0.49) \\
\hline NW-Shaanxi & $156(6.65)$ & $1122(0.25)$ & $1278(0.29)$ \\
\hline NW-Gansu & $100(6.56)$ & $498(0.27)$ & $598(0.32)$ \\
\hline NW-Qinghai & 99 (5.17) & $258(0.29)$ & $357(0.39)$ \\
\hline NW-Ningxia & $70(3.43)$ & $441(0.46)$ & $511(0.52)$ \\
\hline NW-Xinjiang & $200(2.14)$ & $793(0.26)$ & $993(0.31)$ \\
\hline Total & 9721 (3.93) & $45,678(0.25)$ & $55,399(0.30)$ \\
\hline
\end{tabular}

AKI, acute kidney injury; C, Central of China; E, East of China; ICU, intensive care unit; N, North of China; NE, Northeast of China; NW, Northwest of China; S, South of China; SW, Southwest of China. 


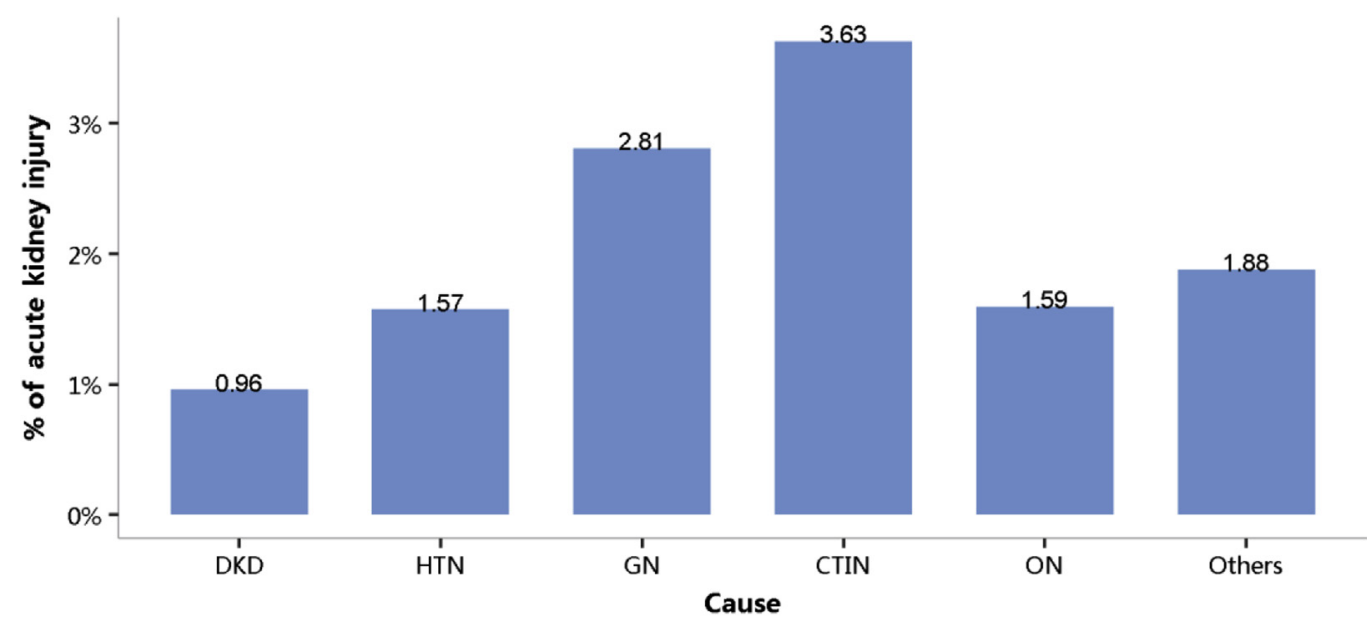

Figure 51 | Percentage of AKI among patients with CKD. AKI, acute kidney injury; CKD, chronic kidney disease; DKD, diabetic kidney disease; HTN, hypertensive nephropathy; GN, glomerulonephritis; CTIN, chronic tubulointerstitial nephropathy; ON, obstructive nephropathy; Others, CKD due to other reasons.

\section{Table 59| Percentage of AKI among patients with CKD, $N(\%)$}

\begin{tabular}{lr}
\hline Cause & AKI \\
\hline DKD & $2296(0.96)$ \\
HTN & $2901(1.57)$ \\
GN & $3756(2.81)$ \\
CTIN & $563(3.63)$ \\
ON & $2204(1.59)$ \\
Others & $3310(1.88)$ \\
Total & $15,030(1.69)$ \\
\hline
\end{tabular}

AKI, acute kidney injury; CKD, chronic kidney disease; DKD, diabetic kidney disease; HTN, hypertensive nephropathy; GN, glomerulonephritis; CTIN, chronic tubulointerstitial nephropathy; ON, obstructive nephropathy; Others, CKD due to other reasons.

\subsection{Percentage of CKD and DM among patients with AKI}

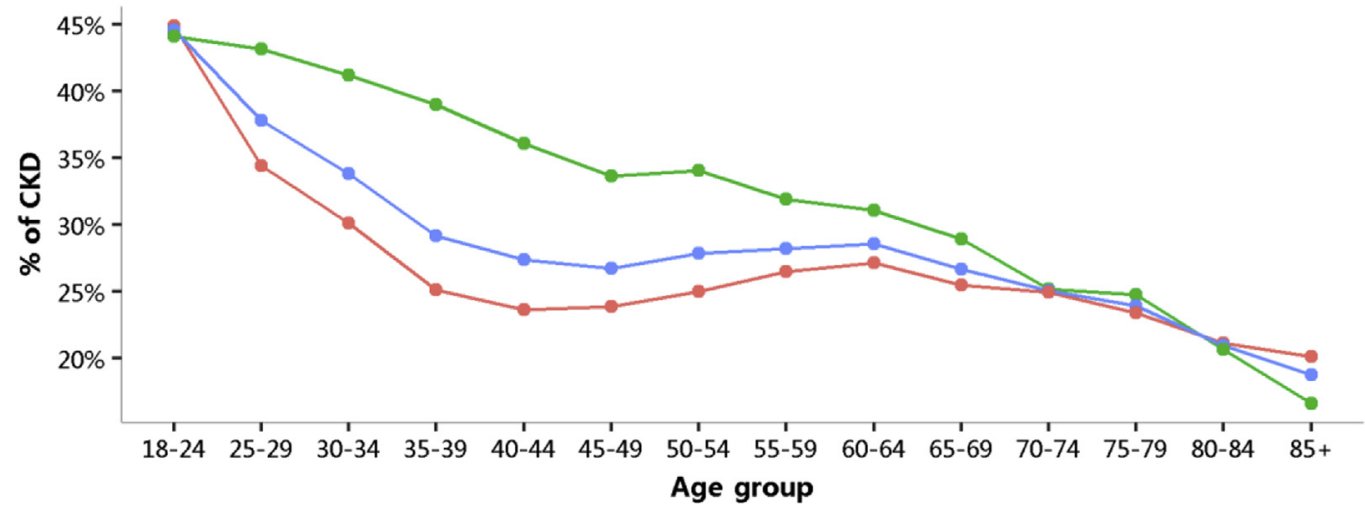

Sex $\bullet$ Male $\bullet$ Female $\bullet$ Total

Figure 52 | Percentage of CKD among patients with AKI, stratified by sex and age. AKl, acute kidney injury; CKD, chronic kidney disease. 
Table 60 | Percentage of CKD among patients with AKI, stratified by sex and age, $\boldsymbol{N}(\%)$

\begin{tabular}{lrrr}
\hline Age group & \multicolumn{1}{c}{ Male } & \multicolumn{1}{c}{ Female } & \\
\hline $18-24$ & $480(44.90)$ & $273(44.10)$ & $753(44.61)$ \\
$25-29$ & $417(34.43)$ & $328(43.16)$ & $745(37.80)$ \\
$30-34$ & $360(30.13)$ & $247(41.24)$ & $607(33.84)$ \\
$35-39$ & $367(25.12)$ & $235(39.04)$ & $602(29.18)$ \\
$40-44$ & $548(23.67)$ & $359(36.12)$ & $907(27.41)$ \\
$45-49$ & $734(23.89)$ & $527(33.62)$ & $1161(26.74)$ \\
$50-54$ & $849(25.01)$ & $525(34.09)$ & $1374(27.85)$ \\
$55-59$ & $910(26.49)$ & $708(31.09)$ & $1437(28.25)$ \\
$60-64$ & $1077(27.14)$ & $577(28.94)$ & $1785(28.58)$ \\
$65-69$ & $917(25.47)$ & $512(25.18)$ & $1494(26.70)$ \\
$70-74$ & $806(24.98)$ & $537(24.77)$ & $1318(25.06)$ \\
$75-79$ & $809(23.43)$ & $369(20.65)$ & $197(16.62)$ \\
$80-84$ & $555(21.13)$ & $5821(29.88)$ & $924(20.93)$ \\
$85+$ & $380(20.11)$ & & $577(18.76)$ \\
Total & $9209(25.64)$ & & $15030(27.13)$ \\
\hline
\end{tabular}

AKI, acute kidney injury; CKD, chronic kidney disease.

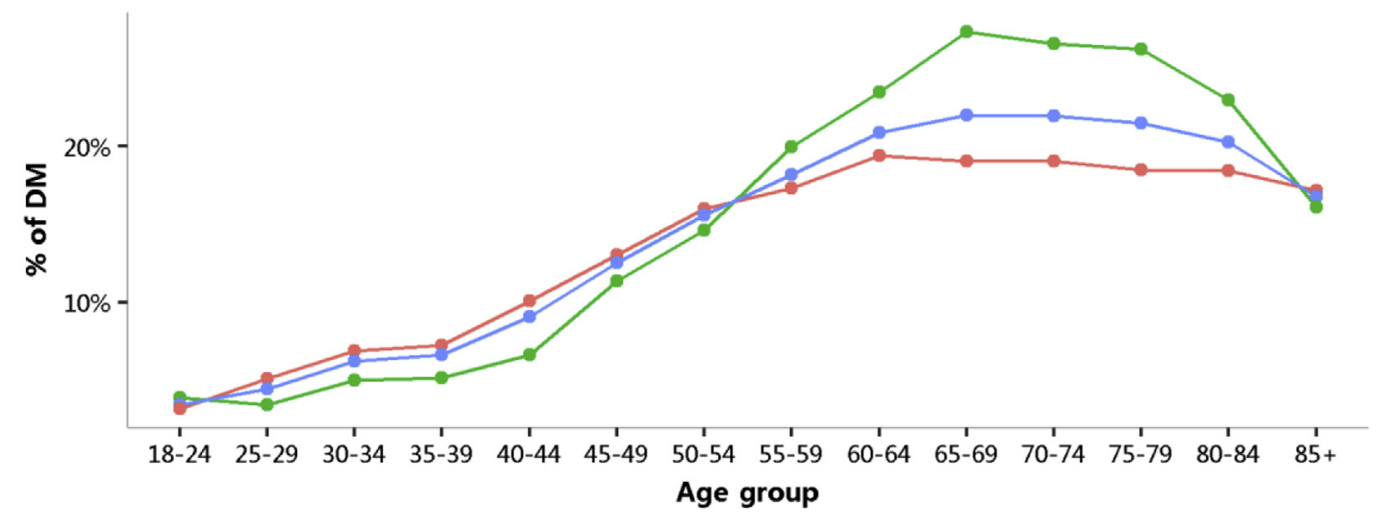

Sex $\bullet$ Male $\bullet$ Female $\bullet$ Total

Figure 53 | Percentage of DM among patients with AKI, stratified by sex and age. AKI, acute kidney injury; DM, diabetes mellitus.

Table 61 | Percentage of DM among patients with AKI, stratified by sex and age, $N$ (\%)

\begin{tabular}{lcrr}
\hline Age group & Male & \multicolumn{1}{c}{ Total } \\
\hline $18-24$ & $34(3.18)$ & $24(3.88)$ & $58(3.44)$ \\
$25-29$ & $62(5.12)$ & $26(3.42)$ & $88(4.46)$ \\
$30-34$ & $82(6.86)$ & $30(5.01)$ & $112(6.24)$ \\
$35-39$ & $106(7.26)$ & $31(5.15)$ & $137(6.64)$ \\
$40-44$ & $234(10.11)$ & $66(6.64)$ & $300(9.07)$ \\
$45-49$ & $401(13.05)$ & $144(11.34)$ & $545(12.55)$ \\
$50-54$ & $543(16.00)$ & $225(14.61)$ & $768(15.57)$ \\
$55-59$ & $594(17.29)$ & $530(19.98)$ & $924(18.16)$ \\
$60-64$ & $770(19.40)$ & $545(27.45)$ & $1304(20.88)$ \\
$65-69$ & $685(19.02)$ & $540(26.56)$ & $1230(21.98)$ \\
$70-74$ & $614(19.03)$ & $568(26.20)$ & $1154(21.94)$ \\
$75-79$ & $639(18.51)$ & $410(22.94)$ & $1207(21.47)$ \\
$80-84$ & $484(18.42)$ & $191(16.12)$ & $894(20.25)$ \\
$85+$ & $324(17.14)$ & $3664(18.81)$ & $515(16.75)$ \\
Total & $5572(15.51)$ &
\end{tabular}

AKI, acute kidney injury; DM, diabetes mellitus. 


\title{
Section II. End-stage kidney disease
}

\section{Chapter 6: Prevalence, incidence, and characteristics of dialysis patients}

\author{
Fang Wang ${ }^{1}$, Chao Yang ${ }^{1}$, Xiaoyu $\mathrm{Sun}^{2}$, Zaiming $\mathrm{Su}^{2}$ and Kunhao Bai ${ }^{3}$ \\ ${ }^{1}$ Renal Division, Department of Medicine, Peking University First Hospital; Peking University Institute of Nephrology, Beijing, China; \\ ${ }^{2}$ Center for Data Science in Health and Medicine, Peking University, Beijing, China; and ${ }^{3}$ Department of Endoscopy, Sun Yat-sen \\ University Cancer Center, State Key Laboratory of Oncology in South China, Collaborative Innovation Center for Cancer Medicine, \\ Guangzhou, China
}

This chapter focuses on the prevalence, demographic characteristics, and treatment modality of dialysis patients in the CHIRA database. Furthermore, the adjusted incidence and mortality rate of dialysis patients were also described based on the CHI database.

In the CHIRA database, the number of dialysis patients was 11,797 , with a male predominance $(56.56 \%)$ (Table 62 ). The mean age of prevalent dialysis patients was 55.0 years (range: 18-97 years) (Table 63), which was younger than that reported from the US and Japan (59.1 and 66.6 years, respectively). ${ }^{5,6}$ For all prevalent dialysis patients, HD was the major modality (90.96\%) (Table 62 and Figure 54).

There was much diversity in the distribution of dialysis patients in different geographic areas. The proportion of dialysis patients in different regions ranged from $0.03 \%$ to $0.39 \%$, with the lowest in Northwest China and the highest in Central China (Table 64). However, interpretation should be cautious because the sampling scheme of CHIRA was not designed for stratified analyses based on geographic regions.
The estimated prevalence of HD and peritoneal dialysis (PD) was 402.18 per million population (PMP) (95\% CI: 394.57-409.79 PMP) and 39.95 PMP (95\% CI: 37.55-42.35 PMP), and the corresponding number of $\mathrm{HD}$ and PD patients was $\sim 553,000$ and $\sim 55,000$ in 2015 , respectively. The ageadjusted incidence rate for dialysis was 122.19 PMP (Table 65). Those numbers are relatively higher than previous data reported for mainland China, ${ }^{7}$ and still lower compared with data from Hong Kong ${ }^{8}$ and Taiwan' (Tables 66 and 67, Figure 55).

The adjusted mortality rate was 28.42 per 1,000 patientyears (Table 68), which increased with age and varied among geographic regions (Tables 69 and 70). The mortality among patients aged 18 to 44 years, 45 to 64 years, and $\geq 65$ years was 11.80 per 1,000 patient-years, 31.57 per 1,000 patient-years, and 91.69 per 1,000 patient-years, respectively (Table 69 and Figure 56). It should be noted that the relatively low mortality rate could be affected by selection bias. The patients covered by commercial insurance in the $\mathrm{CHI}$ database may have a relatively higher socio-economic status and better health consciousness compared with the general population.

Table 62 | Number of dialysis patients, by sex and modality

\begin{tabular}{|c|c|c|c|c|c|c|}
\hline \multirow[b]{2}{*}{ Sex } & \multicolumn{2}{|c|}{ HD } & \multicolumn{2}{|c|}{ PD } & \multicolumn{2}{|c|}{ Total } \\
\hline & $N$ & $\%$ & $N$ & $\%$ & $N$ & $\%$ \\
\hline Male & 6167 & $57.47 \%$ & 505 & $47.37 \%$ & 6672 & $56.56 \%$ \\
\hline Female & 4564 & $42.53 \%$ & 561 & $52.63 \%$ & 5125 & $43.44 \%$ \\
\hline Total & 10,731 & $100 \%$ & 1066 & $100 \%$ & 11,797 & $100 \%$ \\
\hline
\end{tabular}

$H D$, hemodialysis; $P D$, peritoneal dialysis.

Table 63 Number of dialysis patients, by age and modality

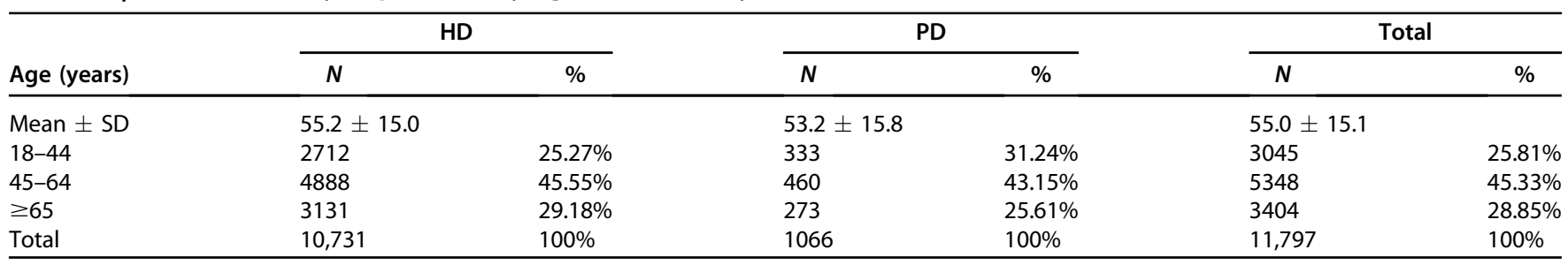

$H D$, hemodialysis; $P D$, peritoneal dialysis. 


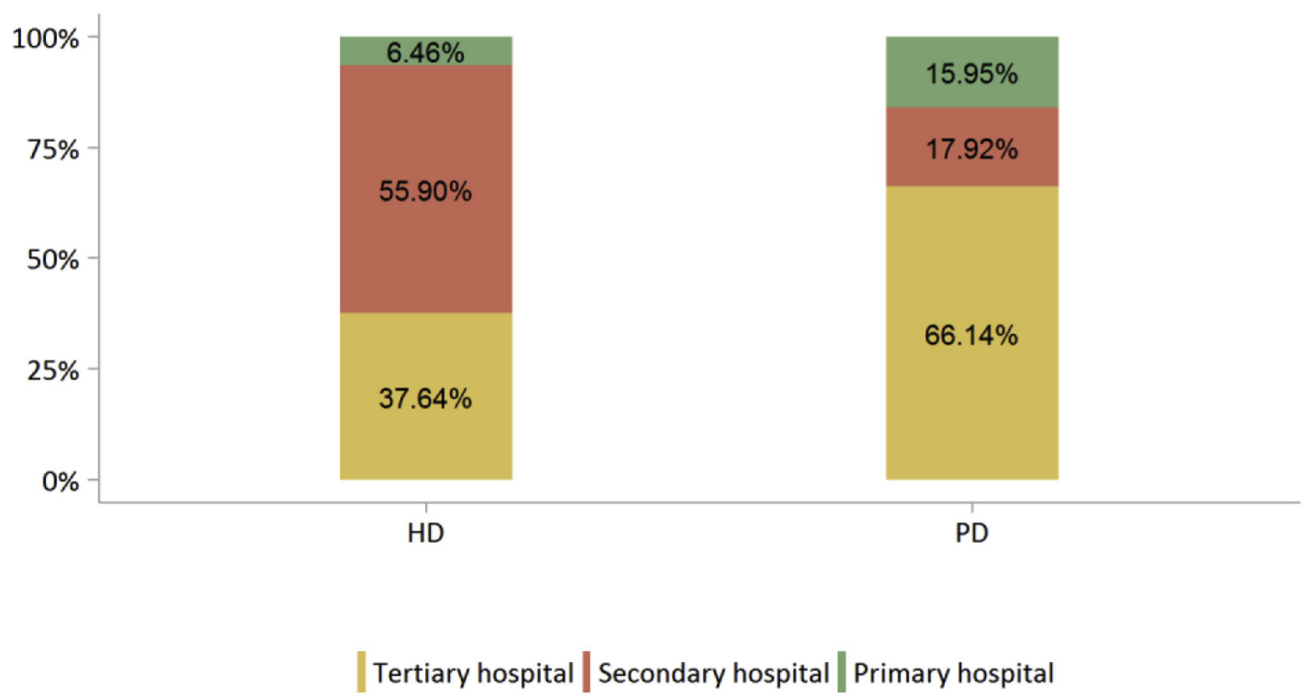

Figure 54| Distribution of HD and PD patients among different hospital grades. HD, hemodialysis; PD, peritoneal dialysis.

Table 64| Number and prevalence of dialysis patients, by geographic region and modality

\begin{tabular}{|c|c|c|c|c|c|c|c|c|c|}
\hline \multirow[b]{2}{*}{ Geographic distribution } & \multicolumn{3}{|c|}{ HD } & \multicolumn{3}{|c|}{ PD } & \multicolumn{3}{|c|}{ Total } \\
\hline & $\mathrm{N}$ & $\%$ & $\overline{\% \text { in } \text { CHIRA }^{a}}$ & $\mathrm{~N}$ & $\%$ & $\overline{\% \text { in } \text { CHIRA }^{a}}$ & $\mathrm{~N}$ & $\%$ & $\%$ in CHIRA \\
\hline East China & 1333 & $12.42 \%$ & $0.13 \%$ & 368 & $34.52 \%$ & $0.04 \%$ & 1701 & $14.42 \%$ & $0.17 \%$ \\
\hline Central China & 1563 & $14.57 \%$ & $0.35 \%$ & 182 & $17.07 \%$ & $0.04 \%$ & 1745 & $14.79 \%$ & $0.39 \%$ \\
\hline South China & 431 & $4.02 \%$ & $0.11 \%$ & 98 & $9.19 \%$ & $0.03 \%$ & 529 & $4.48 \%$ & $0.14 \%$ \\
\hline Northwest China & 178 & $1.66 \%$ & $0.03 \%$ & 29 & $2.72 \%$ & $0.01 \%$ & 207 & $1.75 \%$ & $0.03 \%$ \\
\hline Southwest China & 5932 & $55.28 \%$ & $0.18 \%$ & 143 & $13.41 \%$ & $0.00 \%$ & 6075 & $51.50 \%$ & $0.19 \%$ \\
\hline
\end{tabular}

CHIRA: China Health Insurance Research; HD: hemodialysis; PD: peritoneal dialysis.

aPercentage of dialysis patients in the sampling database of CHIRA in different geographic regions.

Table 65 | Incidence of dialysis patients, by sex

\begin{tabular}{lcccc}
\hline Sex & Incidence count $^{\text {a }}$ & Exposure count (person-years) & Raw incidence rate (PMP) & Adjusted incidence rate (PMP) $^{\text {b }}$ \\
\hline Male & 3965.11 & 28710220.04 & 138.11 & 124.73 \\
Female & 2363.58 & 31053775.49 & 76.11 & 119.24 \\
Total & 6328.69 & 59763995.54 & 105.89 & 122.19 \\
\hline
\end{tabular}

PMP, per million population.

ancidence count had taken into account of incurred but not reported (IBNR).

${ }^{\mathrm{b}}$ Adjusted incidence rate was standardized by the direct method using the 2010 national census population.

Table 66| Incidence of dialysis patients, by age and sex

\begin{tabular}{|c|c|c|c|c|c|c|c|c|c|}
\hline \multirow[b]{2}{*}{$\begin{array}{l}\text { Age } \\
\text { (years) }\end{array}$} & \multicolumn{3}{|c|}{ Male } & \multicolumn{3}{|c|}{ Female } & \multicolumn{3}{|c|}{ Total } \\
\hline & $\begin{array}{l}\text { Incidence } \\
\text { count }^{\mathrm{a}}\end{array}$ & $\begin{array}{l}\text { Exposure count } \\
\text { (person-years) }\end{array}$ & $\begin{array}{l}\text { Incidence } \\
\text { rate (PMP) }\end{array}$ & $\begin{array}{l}\text { Incidence } \\
\text { count }^{\mathrm{a}}\end{array}$ & $\begin{array}{l}\text { Exposure count } \\
\text { (person-years) }\end{array}$ & $\begin{array}{l}\text { Incidence } \\
\text { rate (PMP) }\end{array}$ & $\begin{array}{l}\text { Incidence } \\
\text { count }^{\mathrm{a}}\end{array}$ & $\begin{array}{l}\text { Exposure count } \\
\text { (person-years) }\end{array}$ & $\begin{array}{l}\text { Incidence } \\
\text { rate (PMP) }\end{array}$ \\
\hline $45-64$ & 2415.81 & 12463502.22 & 193.83 & 1529.99 & 14364086.32 & 106.51 & 3945.80 & 26827588.54 & 147.08 \\
\hline$\geq 65$ & 116.10 & 354839.43 & 327.19 & 134.19 & 553563.17 & 242.40 & 250.28 & 908402.61 & 275.52 \\
\hline
\end{tabular}

PMP, per million population.

ancidence count had taken into account of incurred but not reported (IBNR). 


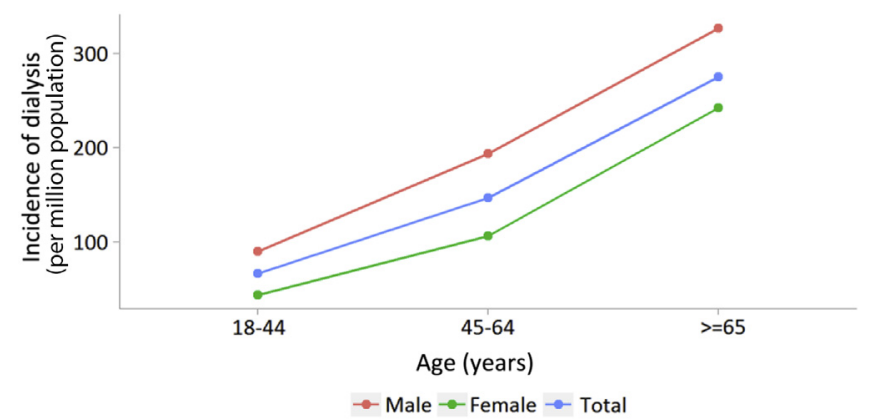

Figure 55 | Incidence of dialysis patients, by age and sex.

Table 67| Incidence of dialysis patients, by geographic distribution and sex

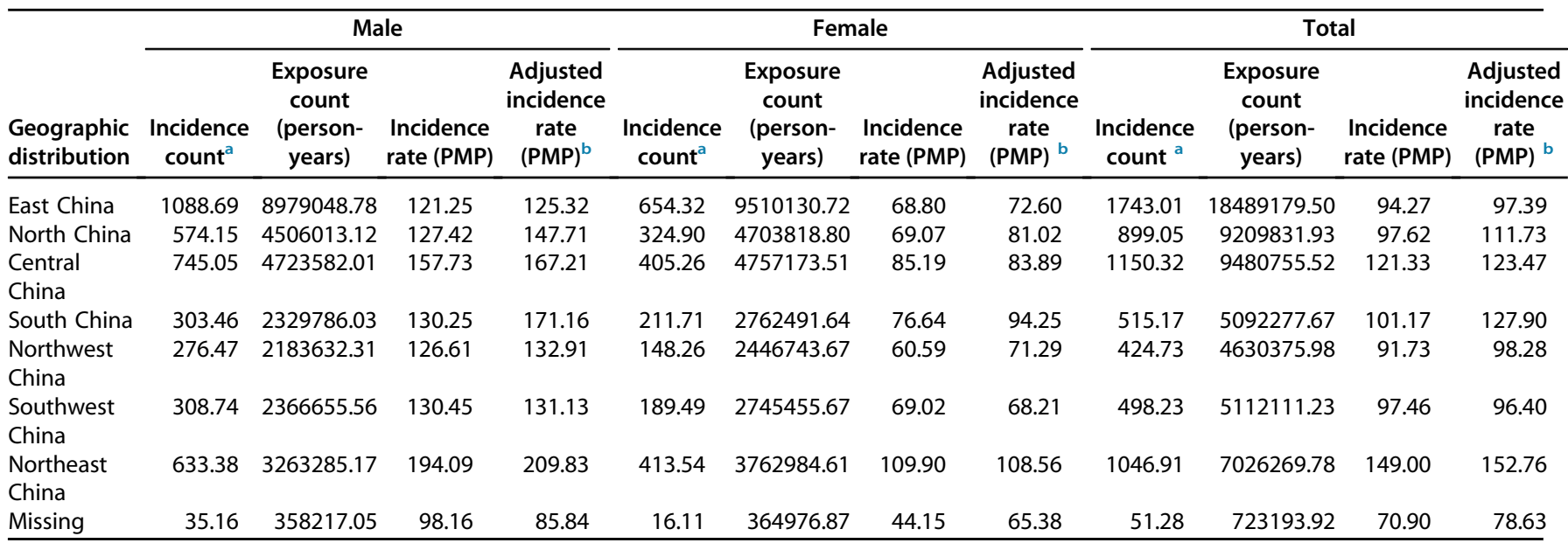

PMP, per million population.

ancidence count had taken into account of incurred but not reported (IBNR).

${ }^{b}$ Adjusted incidence rate was standardized by the direct method using the 2010 national census population.

Table 68| All-cause mortality rate of dialysis patients, by sex

\begin{tabular}{lcccc}
\hline Sex & Death count & Exposure count (patient-years) & Mortality rate (per 1,000 patient-years) & Adjusted mortality rate (per 1,000 patient-years) \\
\hline Male & 380 & 11951.25 & 31.80 & 28.40 \\
Female & 262 & 8462.07 & 30.96 & 28.93 \\
Total & 642 & 20413.32 & 34.08 & 28.42 \\
\hline
\end{tabular}

${ }^{a}$ Adjusted mortality rate was standardized by the direct method using the 2010 national census population.

Table 69| All-cause mortality rate of dialysis patients, by age and sex

\begin{tabular}{|c|c|c|c|c|c|c|c|c|c|}
\hline \multirow[b]{2}{*}{$\begin{array}{l}\text { Age } \\
\text { (years) }\end{array}$} & \multicolumn{3}{|c|}{ Male } & \multicolumn{3}{|c|}{ Female } & \multicolumn{3}{|c|}{ Total } \\
\hline & $\begin{array}{l}\text { Death } \\
\text { count }\end{array}$ & $\begin{array}{l}\text { Exposure count } \\
\text { (patient-years) }\end{array}$ & $\begin{array}{l}\text { Mortality rate (per } \\
1,000 \text { patient-years) }\end{array}$ & $\begin{array}{l}\text { Death } \\
\text { count }\end{array}$ & $\begin{array}{c}\text { Exposure count } \\
\text { (patient-years) }\end{array}$ & $\begin{array}{l}\text { Mortality rate (per } \\
1,000 \text { patient-years) }\end{array}$ & $\begin{array}{l}\text { Death } \\
\text { count }\end{array}$ & $\begin{array}{c}\text { Exposure count } \\
\text { (patient-years) }\end{array}$ & $\begin{array}{l}\text { Mortality rate (per } \\
1,000 \text { patient-years) }\end{array}$ \\
\hline $18-44$ & 34 & 2766.97 & 12.29 & 16 & 1469.71 & 10.89 & 50 & 4236.68 & 11.80 \\
\hline $45-64$ & 284 & 8544.13 & 33.24 & 184 & 6280.19 & 29.30 & 468 & 14824.31 & 31.57 \\
\hline$\geq 65$ & 62 & 640.15 & 96.85 & 62 & 712.18 & 87.06 & 124 & 1352.33 & 91.69 \\
\hline
\end{tabular}




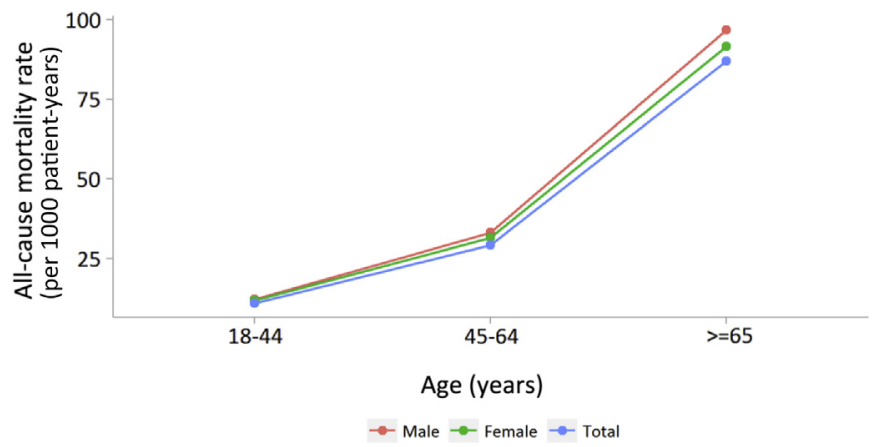

Figure 56 | All-cause mortality rate of dialysis patients, by age and sex.

Table 70| All-cause mortality rate of dialysis patients, by geographic distribution and sex

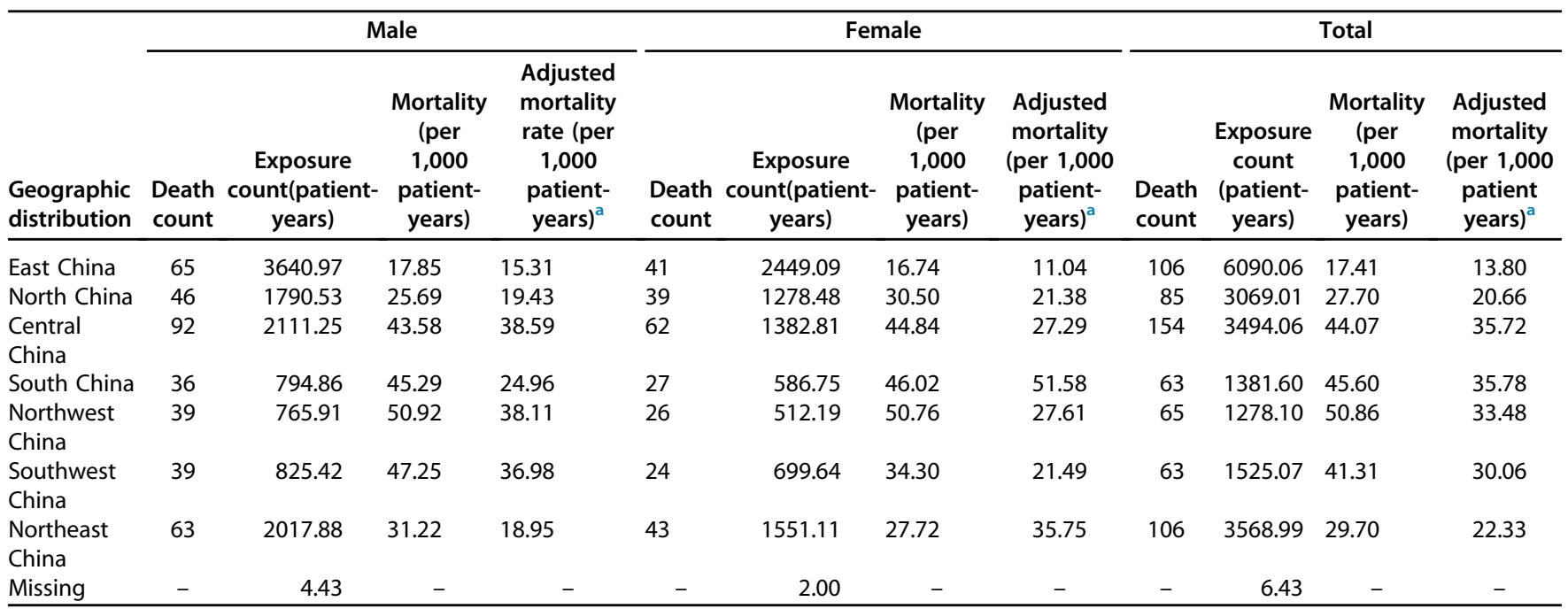

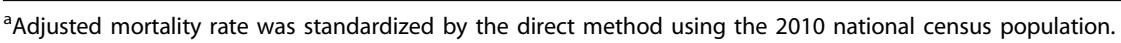




\section{Chapter 7: Clinical measurement and treatment among dialysis patients}

Wen Tang ${ }^{1}$, Song Wang ${ }^{1}$, Hong $\mathrm{Chu}^{2}$, Fang Wang ${ }^{2}$ and Yue Wang ${ }^{1}$

${ }^{1}$ Department of Nephrology, Peking University Third Hospital, Beijing, China; ${ }^{2}$ Renal Division, Department of Medicine, Peking University First Hospital; and Peking University Institute of Nephrology, Beijing, China

This chapter focuses on the monitoring and treatment of several major complications (including anemia, mineral bone disorder, and malnutrition) of dialysis patients.

The percentage of dialysis patients who achieved the recommended monitoring frequency (by Kidney Disease: Improving Global Outcomes guidelines ${ }^{10,11}$ ) for hemoglobin, ferritin, phosphorus, and PTH was 50.00\%, 47.76\%, $42.63 \%$, and $44.39 \%$ for $\mathrm{HD}$, and $59.18 \%, 59.36 \%, 53.18 \%$, and $53.93 \%$ for PD, respectively (Figures 57 and 58). The percentage of erythropoietin, phosphorus binder, and calcitriol use was $71.27 \%, 40.68 \%$, and $59.61 \%$ for $\mathrm{HD}$, and was $59.74 \%, 47.00 \%$ and $60.11 \%$ for PD patients (Figures 59 and 60).

Regarding the monitoring frequency of blood albumin, $31.45 \%$ (HD) and $46.82 \%(\mathrm{PD})$ of patients achieved the recommended goal (Figure 61). For patients with diabetes, the percentage of those who had eye examination, lipid testing, and hemoglobin Alc testing at least once a year was only $5.70 \%$ and $6.49 \%$ for $\mathrm{HD}$ and $\mathrm{PD}$ patients, respectively (Figure 62).
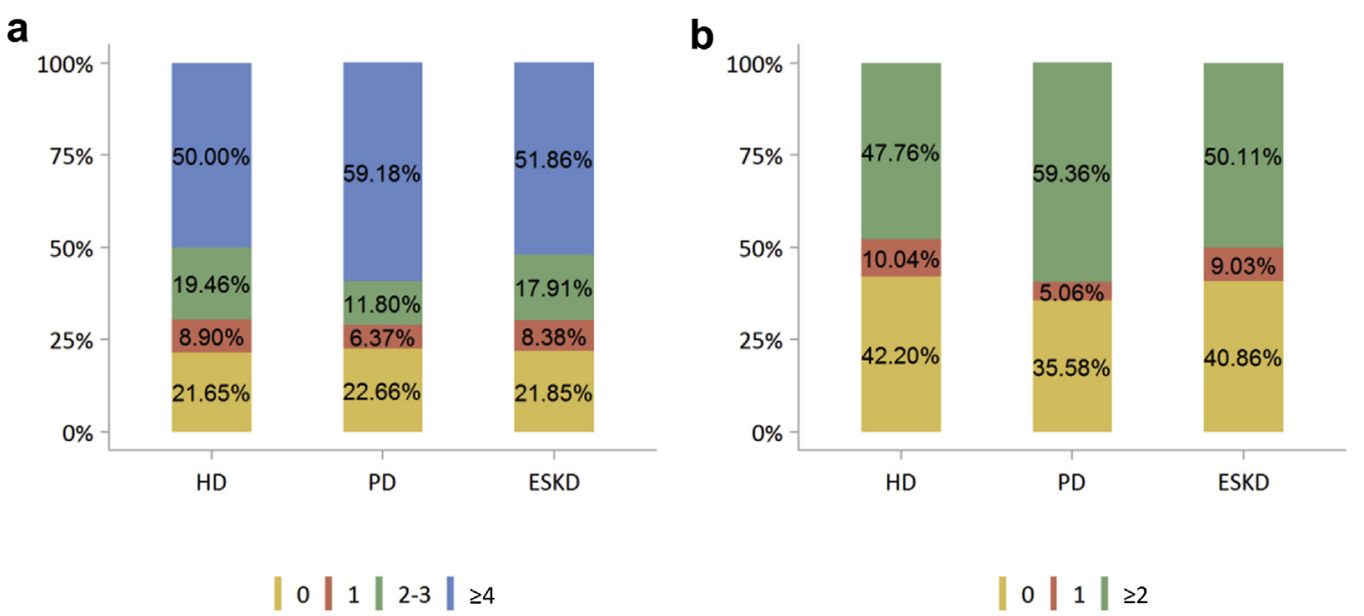

Figure 57| Frequency of (a) hemoglobin and (b) serum ferritin measurement among dialysis patients, by modality. ESKD, end-stage kidney disease; HD, hemodialysis; PD, peritoneal dialysis. 
a

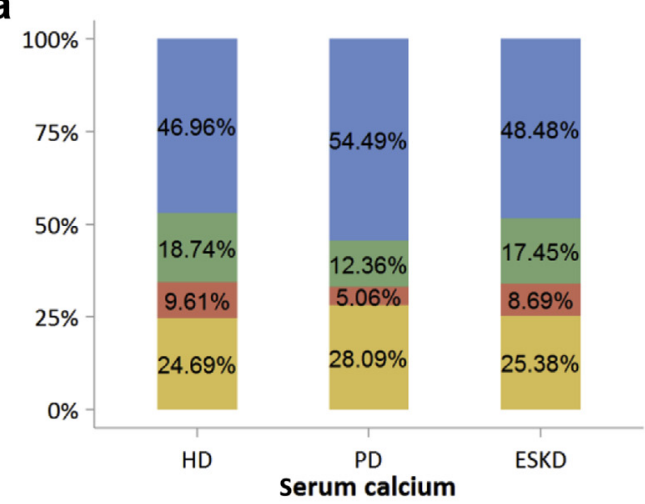

$\| 0|1| 2-3 \mid \geq 4$ b

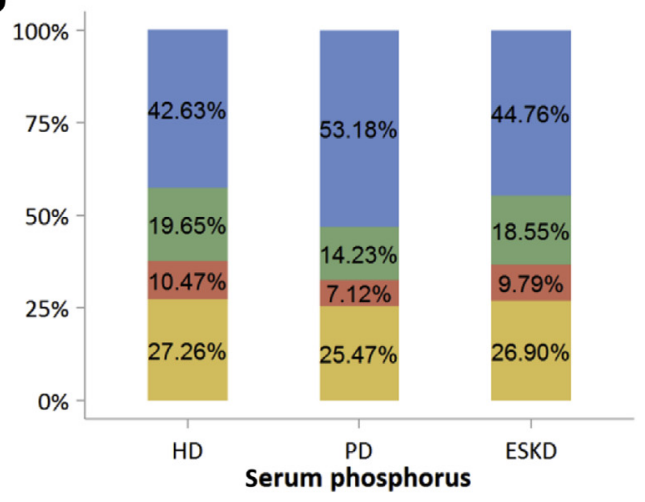

| 0 || $1 \mid$ | 2-3 | $\geq 4$

C

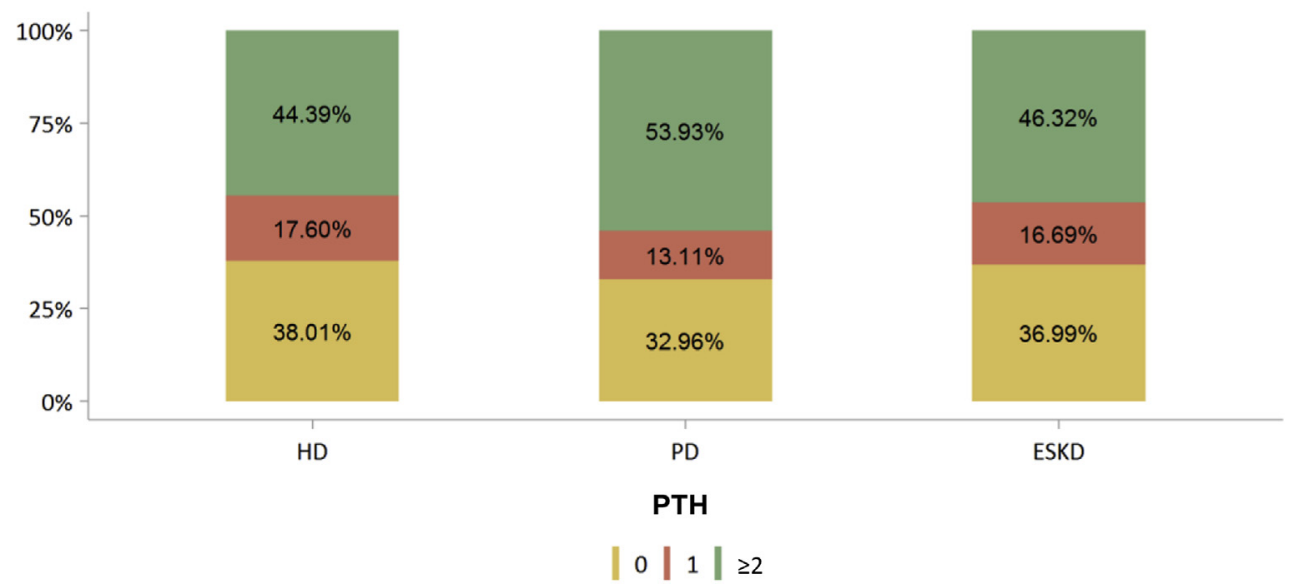

Figure 58 | Percentage of dialysis patients who underwent (a) calcium, (b) phosphorus, and (c) PTH testing per year. ESKD, end-stage kidney disease; HD, hemodialysis; PD, peritoneal dialysis; PTH, parathyroid hormone.

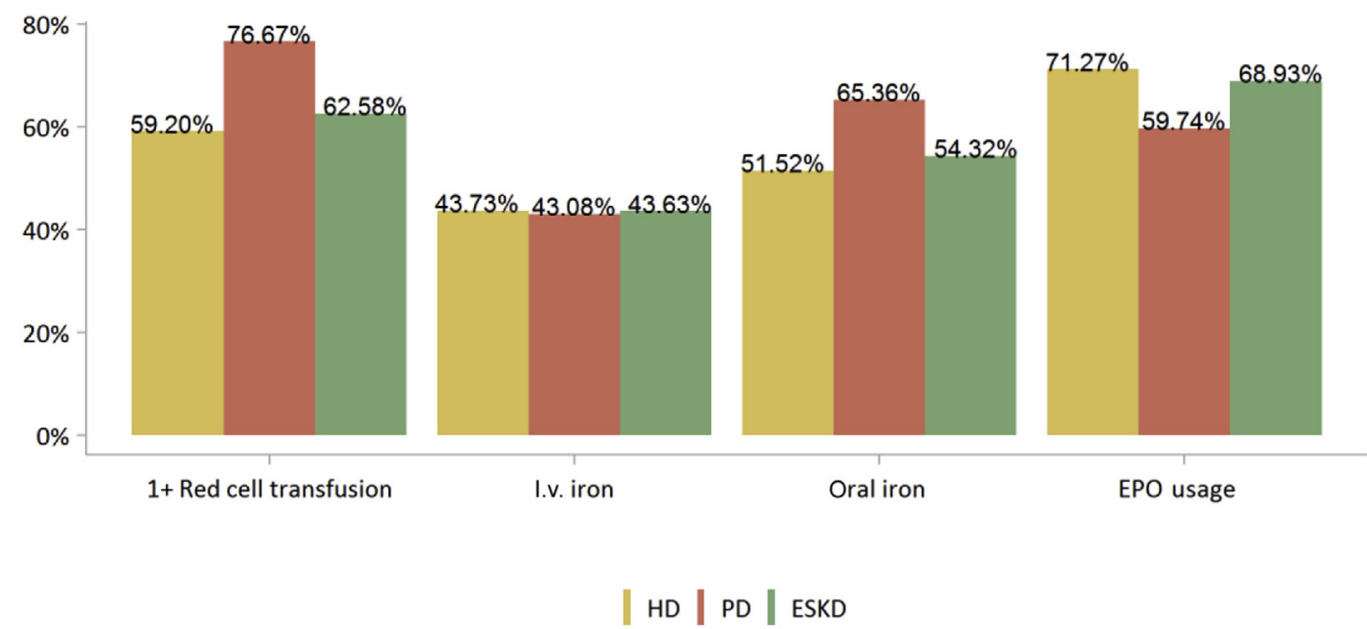

Figure 59| Percentage of dialysis patients received anemia related treatment. EPO, erythropoietin; ESKD, end-stage kidney disease; HD, hemodialysis; PD, peritoneal dialysis. 


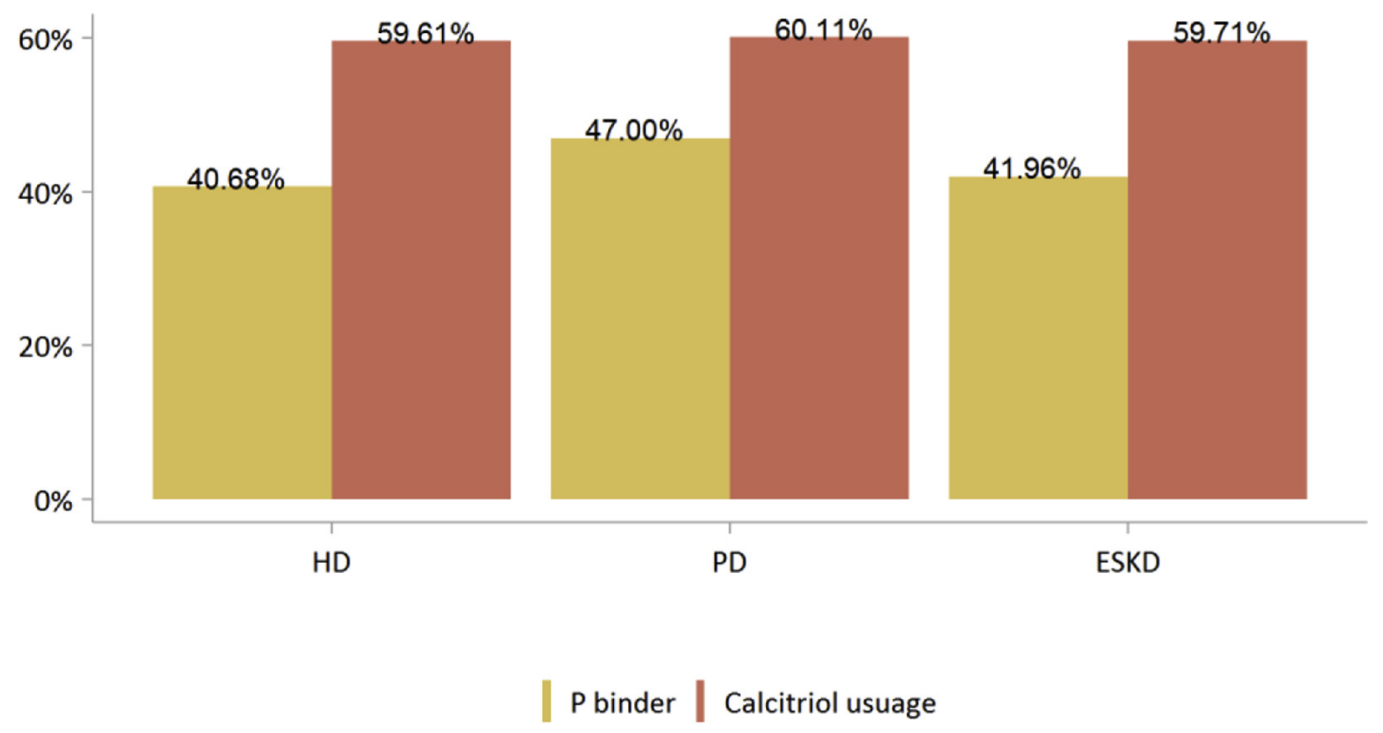

Figure 60 | Percentage of dialysis patients who received MBD-related treatment. ESKD, end-stage kidney disease; HD, hemodialysis; MBD, mineral and bone disorder; PD, peritoneal dialysis.

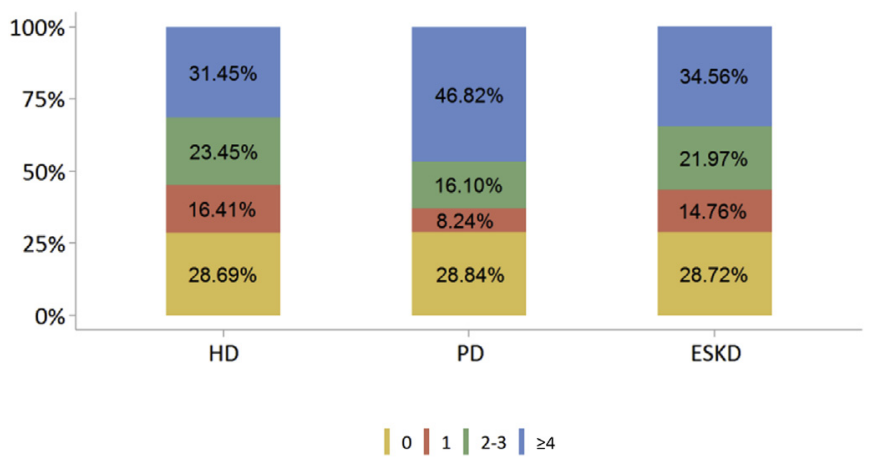

Figure 61 | Percentage of dialysis patients who underwent blood albumin testing. ESKD, end-stage kidney disease; HD, hemodialysis; PD, peritoneal dialysis.

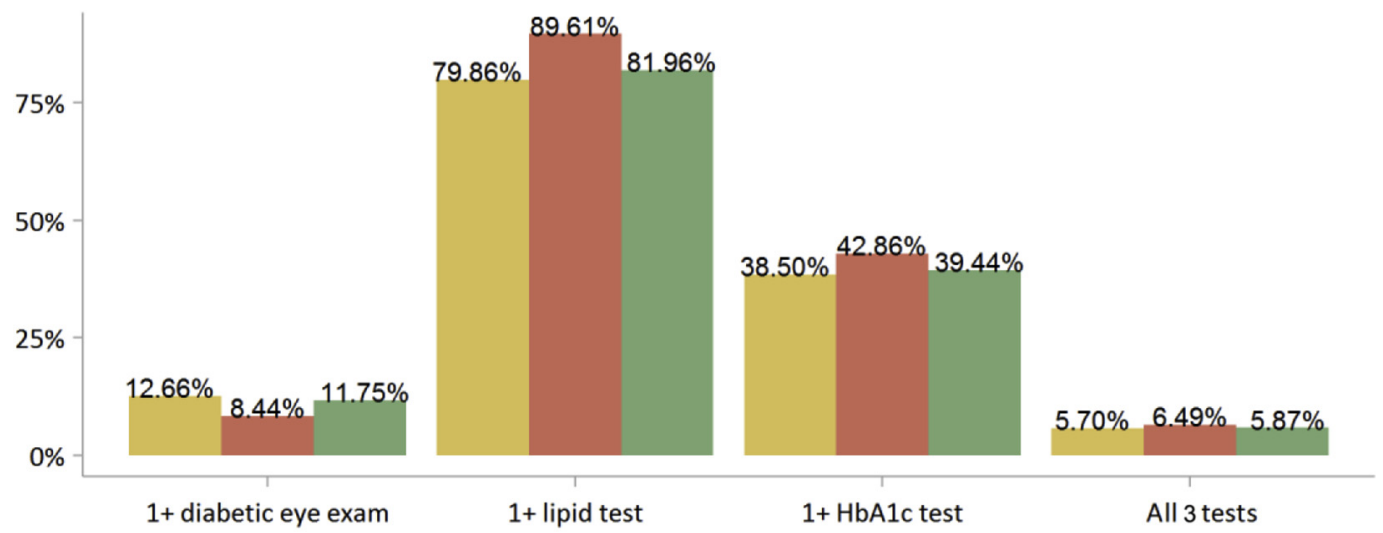

$$
\text { IHD | PD | ESKD }
$$

Figure 62 | Diabetes-related examinations among dialysis patients with diabetes mellitus. ESKD, end-stage kidney disease; HD, hemodialysis; PD, peritoneal dialysis. 


\title{
Chapter 8: Vascular access
}

\author{
Dongliang Zhang ${ }^{1}$, Liren Zheng ${ }^{1}$, Xiaoyu $\mathrm{Sun}^{2}$, Hong $\mathrm{Chu}^{3}$, Fang Wang ${ }^{3}$ and Feng $\mathrm{Yu}^{1,3}$ \\ ${ }^{1}$ Blood Purification Center of Nephrology Department, Peking University International Hospital, Beijing, China; ${ }^{2}$ Center for Data \\ Science in Health and Medicine, Peking University, Beijing, China; and ${ }^{3}$ Renal Division, Department of Medicine, Peking University \\ First Hospital; Peking University Institute of Nephrology, Beijing, China
}

This chapter focuses on vascular access (VA) use of prevalent dialysis patients. AVF or AVG was the predominant type of VA for prevalent HD patients, accounted for $80.48 \%$ (Table 71). The number decreased with age, and the lowest proportion was found in the $65+$ age group $(73.11 \%)$. Moreover, patients with diabetes tended to have a lower proportion of AVF and/or AVG use than patients without diabetes $(57.73 \%$ vs. $84.80 \%)$.

Variation in VA use among different geographic regions was observed (Table 72). Patients in North China had the highest percentage of AVF and/or AVG use (94.61\%) and the lowest percentage of NCC intervention (4.63\%). Patients in Northwest China had the lowest percentage of AVF and/or AVG (62.36\%) and the highest percentage of CVC use (37.64\%), especially TCC (11.24\%). However, the interpretation should be cautious since the sampling scheme of the
CHIRA database was not designed for stratified analyses based on geographic region.

Among 1,066 PD patients, 143 of them (13.41\%) had a new PD catheter placement (Table 73), which indicated that these people were new-onset PD patients. Significant geographic variation of access use was observed among PD patients (Table 74). Patients in North China had the highest percentage of stable PD without intervention for PD catheter $(88.48 \%)$ and the lowest percentage of transient CVC (3.64\%).

The percentage of PD transfer set exchange was only $33.89 \%$. Less than one-quarter $(20.07 \%)$ of patients exchanged PD transfer set twice or more. Patients who were 65 years or older had the lowest percentage of exchange $(30.52 \%)$ (Table 75$)$. Considering the geographic regions, the highest percentage of regular PD transfer set exchange was observed in South China (51.61\%).

Table 71 | Type of vascular access in use among HD patients

\begin{tabular}{|c|c|c|c|c|c|c|c|c|}
\hline & \multicolumn{2}{|c|}{$\begin{array}{l}\text { Tunneled cuffed } \\
\text { catheter }\end{array}$} & \multicolumn{2}{|c|}{ Noncuffed catheter } & \multicolumn{2}{|c|}{$\begin{array}{c}\text { Operations for AVF/ } \\
\text { AVG }\end{array}$} & \multicolumn{2}{|c|}{ Stable AVF/AVG ${ }^{\mathrm{a}}$} \\
\hline & $N$ & $\%$ & $N$ & $\%$ & $N$ & $\%$ & $N$ & $\%$ \\
\hline Male & 104 & $1.69 \%$ & 915 & $14.84 \%$ & 102 & $1.65 \%$ & 5046 & $81.82 \%$ \\
\hline Female & 84 & $1.84 \%$ & 798 & $17.48 \%$ & 92 & $2.02 \%$ & 3590 & $78.66 \%$ \\
\hline \multicolumn{9}{|l|}{ Age (years) } \\
\hline $18-44$ & 23 & $0.85 \%$ & 334 & $12.32 \%$ & 40 & $1.47 \%$ & 2315 & $85.36 \%$ \\
\hline \multicolumn{9}{|l|}{ Diabetes } \\
\hline Yes & 95 & $5.54 \%$ & 545 & $31.78 \%$ & 85 & $4.96 \%$ & 990 & $57.73 \%$ \\
\hline No & 93 & $1.03 \%$ & 1168 & $12.95 \%$ & 109 & $1.21 \%$ & 7646 & $84.80 \%$ \\
\hline Total & 188 & $1.75 \%$ & 1713 & $15.96 \%$ & 194 & $1.81 \%$ & 8636 & $80.48 \%$ \\
\hline
\end{tabular}

AVF, arteriovenous fistula; AVG, arteriovenous graft; $H D$, hemodialysis.

aUsable AVF/AVG without any intervention during 2015.

Table 72 | Geographic variation of vascular access in use among HD patients

\begin{tabular}{|c|c|c|c|c|c|c|c|c|}
\hline & \multicolumn{2}{|c|}{$\begin{array}{l}\text { Tunneled cuffed } \\
\text { catheter }\end{array}$} & \multicolumn{2}{|c|}{ Noncuffed catheter } & \multicolumn{2}{|c|}{$\begin{array}{c}\text { Operations for AV } \\
\text { F/AVG }\end{array}$} & \multicolumn{2}{|c|}{ Stable AVF/AVG ${ }^{a}$} \\
\hline & $N$ & $\%$ & $N$ & $\%$ & $N$ & $\%$ & $N$ & $\%$ \\
\hline Central China & 39 & $2.50 \%$ & 391 & $25.02 \%$ & 111 & $7.10 \%$ & 1022 & $65.39 \%$ \\
\hline East China & 74 & $5.55 \%$ & 285 & $21.38 \%$ & 29 & $2.18 \%$ & 945 & $70.89 \%$ \\
\hline South China & 9 & $2.09 \%$ & 130 & $30.16 \%$ & 8 & $1.86 \%$ & 284 & $65.89 \%$ \\
\hline Northwest China & 20 & $11.24 \%$ & 47 & $26.40 \%$ & 0 & $0.00 \%$ & 111 & $62.36 \%$ \\
\hline Total & 188 & $1.75 \%$ & 1713 & $15.96 \%$ & 194 & $1.81 \%$ & 8636 & $80.48 \%$ \\
\hline
\end{tabular}

AVF, arteriovenous fistula; AVG, arteriovenous graft; $H D$, hemodialysis.

a Usable AVF/AVG without any intervention during 2015. 
Table 73| Type of dialysis access among PD patients, by age and sex

\begin{tabular}{|c|c|c|c|c|c|c|}
\hline & \multicolumn{2}{|c|}{ New PD catheter placement } & \multicolumn{2}{|c|}{ Transitional CVC } & \multicolumn{2}{|c|}{ No PD catheter operations } \\
\hline & $N$ & $\%$ & $N$ & $\%$ & $N$ & $\%$ \\
\hline \multicolumn{7}{|l|}{ Sex } \\
\hline \multicolumn{7}{|l|}{ Age (years) } \\
\hline $18-44$ & 40 & $12.01 \%$ & 58 & $17.42 \%$ & 235 & $70.57 \%$ \\
\hline $45-65$ & 61 & $13.26 \%$ & 88 & $19.13 \%$ & 311 & $67.61 \%$ \\
\hline
\end{tabular}

CVC, central venous catheters; PD, peritoneal dialysis.

Table 74| Geographic variation of dialysis access among PD patients

\begin{tabular}{|c|c|c|c|c|c|c|c|c|}
\hline & \multicolumn{2}{|c|}{$\begin{array}{c}\text { New PD catheter } \\
\text { placement }\end{array}$} & \multicolumn{2}{|c|}{ Transitional CVC } & \multicolumn{3}{|c|}{ No PD catheter operations } & \multirow{2}{*}{$\frac{\text { Total }}{\%}$} \\
\hline & $N$ & $\%$ & $N$ & $\%$ & $N$ & $\%$ & $N$ & \\
\hline North China & 13 & $7.88 \%$ & 6 & $3.64 \%$ & 146 & $88.48 \%$ & 165 & $15.48 \%$ \\
\hline Central China & 22 & $12.09 \%$ & 92 & $50.55 \%$ & 68 & $37.36 \%$ & 182 & $17.07 \%$ \\
\hline East China & 59 & $16.03 \%$ & 55 & $14.95 \%$ & 254 & $69.02 \%$ & 368 & $34.52 \%$ \\
\hline South China & 18 & $18.37 \%$ & 18 & $18.37 \%$ & 62 & $63.27 \%$ & 98 & $9.19 \%$ \\
\hline Northwest China & 3 & $10.34 \%$ & 6 & $20.69 \%$ & 20 & $68.97 \%$ & 29 & $2.72 \%$ \\
\hline Southwest China & 14 & $9.79 \%$ & 12 & $8.39 \%$ & 117 & $81.82 \%$ & 143 & $13.41 \%$ \\
\hline Northeast China & 14 & $17.28 \%$ & 17 & $20.99 \%$ & 50 & $61.73 \%$ & 81 & $7.60 \%$ \\
\hline Total & 143 & $13.41 \%$ & 206 & $19.32 \%$ & 717 & $67.26 \%$ & 1066 & $100.00 \%$ \\
\hline
\end{tabular}

CVC, central venous catheters; PD, peritoneal dialysis.

Table 75 | Frequency of PD transfer set exchange among prevalent PD patients

\begin{tabular}{|c|c|c|c|c|c|c|}
\hline & \multicolumn{2}{|c|}{ No replacement } & \multicolumn{2}{|c|}{ Once } & \multicolumn{2}{|c|}{$\geq 2$ times } \\
\hline & $N$ & $\%$ & $N$ & $\%$ & $N$ & $\%$ \\
\hline \multicolumn{7}{|l|}{ Sex } \\
\hline Female & 174 & $69.32 \%$ & 30 & $11.95 \%$ & 47 & $18.73 \%$ \\
\hline \multicolumn{7}{|l|}{ Age (years) } \\
\hline $18-44$ & 90 & $63.38 \%$ & 22 & $15.49 \%$ & 30 & $21.13 \%$ \\
\hline $45-65$ & 162 & $68.07 \%$ & 27 & $11.34 \%$ & 49 & $20.59 \%$ \\
\hline
\end{tabular}

$\mathrm{PD}$, peritoneal dialysis. 


\title{
Chapter 9: Cardiovascular diseases and diabetes among dialysis patients
}

\author{
Xinju Zhao ${ }^{1}$, Hong $\mathrm{Chu}^{2}$, Zaiming $\mathrm{Su}^{3}$, Fang Wang ${ }^{2}$ and Li Zuo ${ }^{1}$ \\ ${ }^{1}$ Department of Nephrology, Peking University People's Hospital, Beijing, China; ${ }^{2}$ Renal Division, Department of Medicine, Peking \\ University First Hospital; Peking University Institute of Nephrology, Beijing, China; and ${ }^{3}$ Center for Data Science in Health and \\ Medicine, Peking University, Beijing, China
}

Patients receiving dialysis therapy have an increased risk of developing cardiovascular diseases (CVDs), which are the leading cause of death in dialysis patients. DKD is the leading cause of ESKD in developed countries, and also the leading cause of CKD in China. In this chapter, we provide an overview of CVDs and diabetes among adult dialysis patients, stratified by age, sex, and treatment modalities.

As expected, CVDs were common in ESKD patients, with a prevalence of $45.49 \%$ in adult patients on dialysis in 2015. The prevalence of CVDs was similar between dialysis patients who received HD (45.72\%) and PD (44.57\%), respectively; it was comparable between male (44.99\%) and female $(46.18 \%)$ patients, and it increased with age $(32.97 \%, 47.21 \%$, and $53.01 \%$ for patients aged $18-44,45-64$, and $\geq 65$ years, respectively) (Table 76).

ESKD patients receiving dialysis had a high burden of CVDs across a wide range of conditions, including CHD, heart failure (HF), cerebrovascular accident and/or transient ischemic attack (CVA/TIA), acute myocardial infarction (AMI), peripheral arterial disease (PAD), and atrial fibrillation (AF). $\mathrm{CHD}$ and $\mathrm{HF}$ were the 2 major leading CVDs $(41.08 \%$ and $10.24 \%$, respectively), while CVA/TIA, AMI, PAD, and AF were less common $(4.17 \%$, $0.76 \%, 0.57 \%$, and $0.30 \%$ respectively). Notably, only $0.23 \%$ and $0.27 \%$ of patients underwent PCI and received pacemaker or implantable cardioverter defibrillators, respectively.

The prevalence of diabetes in dialysis patients was $27.12 \%$, similar between patients who received HD (26.69\%) and PD $(28.84 \%)$. In addition, diabetes occurred predominantly among middle-aged and elderly patients on dialysis (50.35\% and $39.86 \%$ for patients aged $45-64$ and $\geq 65$ years, respectively), while for patients aged 18 to 44 years, the percentage was only $9.79 \%$ (Table 77 ).

Table 76| Prevalence of CVDs in dialysis patients, by modality, age, and sex

\begin{tabular}{llll}
\hline CVDs & HD & PD & \\
\hline Sex & & & Total \\
$\quad$ Male & $44.61 \%$ & $46.64 \%$ & \\
$\quad$ Female & $47.35 \%$ & $42.23 \%$ & \\
Age group (years) & & & \\
$18-44$ & $32.53 \%$ & $34.51 \%$ & \\
$45-64$ & $47.76 \%$ & $44.96 \%$ & $32.97 \%$ \\
$\geq 65$ & $52.95 \%$ & $53.25 \%$ & $47.21 \%$ \\
Total & $45.72 \%$ & $44.57 \%$ & $53.01 \%$ \\
\hline
\end{tabular}

CVDs, cardiovascular diseases; $\mathrm{HD}$, hemodialysis; PD, peritoneal dialysis.

Table 77| Prevalence of diabetes in dialysis patients, by modality, age, and sex

\begin{tabular}{llll}
\hline Diabetes Mellitus & HD & PD & Total \\
\hline Sex & & & \\
$\quad$ Male & $62.21 \%$ & $57.14 \%$ & \\
$\quad$ Female & $37.79 \%$ & $42.86 \%$ & \\
Age group (years) & & & \\
$18-44$ & $8.56 \%$ & $14.29 \%$ & \\
$45-64$ & $51.34 \%$ & $46.75 \%$ & $9.79 \%$ \\
$\geq 65$ & $40.11 \%$ & $38.96 \%$ & $30.35 \%$ \\
Total & $26.69 \%$ & $28.84 \%$ & $39.86 \%$ \\
\hline
\end{tabular}

$\mathrm{HD}$, hemodialysis; PD, peritoneal dialysis. 


\title{
Chapter 10: Hospitalization
}

\author{
Kunhao Bai ${ }^{1}$, Zaiming $\mathrm{Su}^{2}$, Chao Yang ${ }^{3}$, Jinwei Wang ${ }^{3}$ and Fang Wang ${ }^{3}$
}

${ }^{1}$ Department of Endoscopy, Sun Yat-sen University Cancer Center, State Key Laboratory of Oncology in South China, Collaborative Innovation Center for Cancer Medicine, Guangzhou, China; ${ }^{2}$ Center for Data Science in Health and Medicine, Peking University, Beijing, China; and ${ }^{3}$ Renal Division, Department of Medicine, Peking University First Hospital; Peking University Institute of Nephrology, Beijing, China

Hospital admissions and readmissions among ESKD patients represent important indicators regarding the quality of care as well as the health care resource utilization, which is relevant for patients, dialysis facilities, hospitals, and insurers. It has been reported that hospitalization was associated with more severe comorbidities, poorer outcomes, and higher medical expenditure. ${ }^{12}$ In this chapter, we focused on admission rate, length of stay, and rehospitalization within 30 days.

Overall, the all-cause hospitalization rate of admission for dialysis patients was 1.78 per person per year (PPPY) (Table 78), which was similar to that reported in the 2017 United States Renal Data System (USRDS) Annual Data Report (1.7 PPPY). ${ }^{13}$ The overall length of stay PPPY for dialysis patients in 2015 was 29.22 days; it was similar between male and female patients, longer in PD patients than in HD patients (38.78 days vs. 26.87 days), and increased with age (Table 79 ).

PD patients had a higher all-cause hospitalization rate (2.06 PPPY vs. $1.71 \mathrm{PPPY}$ ) and longer length of stay than did HD patients. In addition, dialysis patients with diabetes tended to have a higher all-cause hospitalization rate (2.43 PPPY vs. 1.53 PPPY for all dialysis patients, 2.28 PPPY vs. 1.50 PPPY for HD patients, and 3.01 PPPY vs. 1.67 PPPY for PD patients) and longer length of stay (44.04 days vs. 23.68 days for all dialysis patients, 38.64 days vs. 22.59 days for HD patients, and 63.98 days vs. 28.29 days for PD patients). The hospitalization rate in tertiary hospitals was lower than that in secondary and primary hospitals, for both PD and HD patients (Tables 78 and 79).

For cause-specific hospitalization among patients receiving HD, CVDs were more common than infectious diseases or vascular access events, accounting for $11.07 \%$ of all admissions (Table 80). For PD patients, access events were more common than CVDs and infectious diseases, accounting for $24.93 \%$ of all admissions (Table 81 ). The hospitalization rate for CVDs and infectious diseases in tertiary hospitals was lower than that in secondary and primary hospitals, for both PD and HD patients (Tables 80 and 81).

The rehospitalization rate within 30 days for dialysis patients was $23.18 \%$, and was higher among HD patients than among PD patients $(23.86 \%$ vs. $19.33 \%)$. For HD patients, CVDs were the most common cause of rehospitalization (30.01\%), whereas for PD patients, infectious diseases were the most common cause (26.42\%) (Table 82). The in-hospital mortality rate was $0.50 \%$ for all dialysis patients, $0.56 \%$ for HD patients, and $0.15 \%$ for PD patients, respectively. The percentage of ICU admission was $0.95 \%$ for all dialysis patients, $0.93 \%$ for HD, patients and $1.04 \%$ for PD patients, respectively.

Table 78 | All-cause hospitalization rate for dialysis patients, by modality

\begin{tabular}{|c|c|c|c|c|c|c|}
\hline & \multicolumn{2}{|c|}{ HD } & \multicolumn{2}{|c|}{ PD } & \multicolumn{2}{|c|}{ Total } \\
\hline & Mean & SD & Mean & SD & Mean & $\overline{S D}$ \\
\hline \multicolumn{7}{|l|}{ Sex } \\
\hline Female & 1.74 & 2.71 & 1.91 & 2.51 & 1.77 & 2.67 \\
\hline \multicolumn{7}{|l|}{ Age group (years) } \\
\hline $18-44$ & 1.38 & 2.29 & 1.82 & 2.67 & 1.47 & 2.38 \\
\hline $45-64$ & 1.75 & 2.68 & 1.94 & 2.47 & 1.79 & 2.64 \\
\hline Yes & 2.28 & 2.84 & 3.01 & 2.59 & 2.43 & 2.81 \\
\hline No & 1.50 & 2.52 & 1.67 & 2.47 & 1.53 & 2.51 \\
\hline \multicolumn{7}{|l|}{ Hospital grade } \\
\hline Tertiary hospital & 1.14 & 1.86 & 1.64 & 2.01 & 1.17 & 1.88 \\
\hline Secondary hospital & 1.82 & 2.60 & 3.38 & 3.04 & 1.85 & 2.62 \\
\hline Primary hospital & 1.90 & 2.50 & 1.79 & 1.85 & 1.89 & 2.46 \\
\hline
\end{tabular}

$\mathrm{HD}$, hemodialysis; PD, peritoneal dialysis; PPPY, per person per year. 
Table 79| Length of stay for dialysis patients, by modality

\begin{tabular}{|c|c|c|c|c|c|c|}
\hline & \multicolumn{2}{|c|}{ HD } & \multicolumn{2}{|c|}{ PD } & \multicolumn{2}{|c|}{ Total } \\
\hline & Mean & SD & Mean & SD & Mean & SD \\
\hline \multicolumn{7}{|l|}{ Sex } \\
\hline Female & 26.44 & 39.69 & 39.18 & 64.80 & 29.27 & 46.73 \\
\hline \multicolumn{7}{|l|}{ Age group (years) } \\
\hline $18-44$ & 19.86 & 31.25 & 27.50 & 36.03 & 21.46 & 32.43 \\
\hline $45-64$ & 27.07 & 41.28 & 37.89 & 64.62 & 29.17 & 46.88 \\
\hline Yes & 38.64 & 50.27 & 63.98 & 81.86 & 44.04 & 59.30 \\
\hline No & 22.59 & 35.10 & 28.29 & 47.41 & 23.68 & 37.82 \\
\hline \multicolumn{7}{|l|}{ Hospital grade } \\
\hline Tertiary hospital & 20.91 & 32.23 & 26.66 & 32.54 & 21.23 & 32.27 \\
\hline Secondary hospital & 29.21 & 42.26 & 51.79 & 46.49 & 29.61 & 42.44 \\
\hline Primary hospital & 29.69 & 44.04 & 28.31 & 38.35 & 29.61 & 43.71 \\
\hline
\end{tabular}

$H D$, hemodialysis; PD, peritoneal dialysis; PPPY, per person per year.

Table 80| Percentage of cause-specific hospitalization in HD patients

\begin{tabular}{|c|c|c|c|c|c|c|}
\hline & \multicolumn{2}{|c|}{ CVD } & \multicolumn{2}{|c|}{ Infectious disease } & \multicolumn{2}{|c|}{ Access event } \\
\hline & $N$ & $\%$ & $N$ & $\%$ & $N$ & $\%$ \\
\hline \multicolumn{7}{|l|}{ Sex } \\
\hline Female & 355 & $10.91 \%$ & 198 & $6.08 \%$ & 183 & $5.62 \%$ \\
\hline \multicolumn{7}{|l|}{ Age group (years) } \\
\hline $18-44$ & 112 & $7.44 \%$ & 92 & $6.11 \%$ & 71 & $4.71 \%$ \\
\hline \multicolumn{7}{|l|}{ Diabetes } \\
\hline Yes & 167 & $9.48 \%$ & 81 & $4.60 \%$ & 130 & $7.38 \%$ \\
\hline No & 676 & $11.55 \%$ & 362 & $6.18 \%$ & 284 & $4.85 \%$ \\
\hline \multicolumn{7}{|l|}{ Hospital grade } \\
\hline Tertiary hospital & 217 & $6.52 \%$ & 128 & $3.85 \%$ & 218 & $6.55 \%$ \\
\hline Secondary hospital & 574 & $15.73 \%$ & 264 & $7.23 \%$ & 157 & $4.30 \%$ \\
\hline Primary hospital & 52 & $8.13 \%$ & 51 & $7.97 \%$ & 39 & $6.09 \%$ \\
\hline
\end{tabular}

CVD, cardiovascular disease; $H D$, hemodialysis.

Table 81 | Percentage of cause-specific hospitalization in PD patients

\begin{tabular}{|c|c|c|c|c|c|c|}
\hline & \multicolumn{2}{|c|}{ CVD } & \multicolumn{2}{|c|}{ Infectious disease } & \multicolumn{2}{|c|}{ Access event } \\
\hline & $N$ & $\%$ & $N$ & $\%$ & $N$ & $\%$ \\
\hline \multicolumn{7}{|l|}{ Sex } \\
\hline Female & 23 & $3.32 \%$ & 27 & $3.90 \%$ & 169 & $24.39 \%$ \\
\hline \multicolumn{7}{|l|}{ Age group (years) } \\
\hline $18-44$ & 6 & $1.74 \%$ & 11 & $3.19 \%$ & 133 & $38.55 \%$ \\
\hline Yes & 11 & $3.25 \%$ & 12 & $3.55 \%$ & 75 & $22.19 \%$ \\
\hline No & 32 & $3.19 \%$ & 41 & $4.09 \%$ & 259 & $25.85 \%$ \\
\hline \multicolumn{7}{|l|}{ Hospital grade } \\
\hline Tertiary hospital & 18 & $1.97 \%$ & 15 & $1.64 \%$ & 273 & $29.84 \%$ \\
\hline Secondary hospital & 17 & $5.92 \%$ & 18 & $6.27 \%$ & 46 & $16.03 \%$ \\
\hline Primary hospital & 8 & $5.80 \%$ & 20 & $14.49 \%$ & 15 & $10.87 \%$ \\
\hline
\end{tabular}

CVD, cardiovascular disease; PD, peritoneal dialysis. 
Table 82 | Re-hospitalization rate within 30 days for dialysis patients, by modality

\begin{tabular}{|c|c|c|c|c|c|c|}
\hline & \multicolumn{2}{|c|}{ HD } & \multicolumn{2}{|c|}{ PD } & \multicolumn{2}{|c|}{ Total } \\
\hline & $N$ & $\%$ & $N$ & $\%$ & $N$ & $\%$ \\
\hline \multicolumn{7}{|l|}{ Sex } \\
\hline Female & 806 & $24.77 \%$ & 131 & $18.90 \%$ & 937 & $23.74 \%$ \\
\hline \multicolumn{7}{|l|}{ Age group (years) } \\
\hline $18-44$ & 322 & $21.38 \%$ & 50 & $14.49 \%$ & 372 & $20.10 \%$ \\
\hline $45-64$ & 868 & $25.23 \%$ & 118 & $20.56 \%$ & 986 & $24.56 \%$ \\
\hline CVDs & 253 & $30.01 \%$ & 5 & $11.63 \%$ & 258 & $29.12 \%$ \\
\hline Infectious disease & 89 & $20.09 \%$ & 14 & $26.42 \%$ & 103 & $20.77 \%$ \\
\hline Access events & 119 & $28.74 \%$ & 40 & $11.98 \%$ & 159 & $21.26 \%$ \\
\hline \multicolumn{7}{|l|}{ Diabetes } \\
\hline Yes & 418 & $23.72 \%$ & 75 & $22.19 \%$ & 493 & $23.48 \%$ \\
\hline No & 1399 & $23.90 \%$ & 184 & $18.36 \%$ & 1583 & $23.09 \%$ \\
\hline
\end{tabular}

CVD, cardiovascular disease; HD, hemodialysis; PD, peritoneal dialysis. 


\title{
Chapter 11: Medical expenditures for dialysis patients
}

\author{
Fang Wang ${ }^{1}$, Kunhao $\mathrm{Bai}^{2}$, Chao Yang ${ }^{1}$ and Zaiming $\mathrm{Su}^{3}$ \\ ${ }^{1}$ Renal Division, Department of Medicine, Peking University First Hospital; Peking University Institute of Nephrology, Beijing, China; \\ ${ }^{2}$ Department of Endoscopy, Sun Yat-sen University Cancer Center, State Key Laboratory of Oncology in South China, Collaborative \\ Innovation Center for Cancer Medicine, Guangzhou, China; and ${ }^{3}$ Center for Data Science in Health and Medicine, Peking Uni- \\ versity, Beijing, China
}

Although ESKD patients constitute a small percentage of the population, they consume a disproportionate share of the medical budget (so-called medical expenditure multiplier). In this chapter, we present the patterns of medical expenditure in dialysis patients, as well as its impact on health care resources.

Overall, the number of HD and PD patients constituted only $0.16 \%$ and $0.02 \%$ of individuals covered by UBMI, while they consumed $2.08 \%$ and $0.34 \%$ of the overall UBMI expenditures. The total medical expenditure for dialysis patients was 429 million RMB in 2015, of which $76.61 \%$ was covered by UBMI. The direct cost of dialysis procedure was the leading expenditure for both HD and PD patients $(46.49 \%$ and $42.51 \%)$, followed by drugs ( $25.97 \%$ and $31.07 \%$ ) (Table 83 ).

The median annual overall cost per patient for PD patients was lower than that of HD patients (73,266 RMB vs. 87,125 RMB) (Table 84). However, among inpatients undergoing dialysis, the median cost for PD patients was remarkably higher than that for HD patients $(42,902$ RMB vs. 21,336 RMB) (Table 84).

The proportion of inpatient costs for PD patients was higher than that of HD patients $(47.88 \%$ vs. $42.88 \%)$. The leading inpatient cost for dialysis patients was drug related, accounting for $33.76 \%$ of HD inpatients and $34.45 \%$ of PD inpatients. The proportion of costs covered by UBMI was $70.15 \%$ (70.47\% for HD patients and $68.42 \%$ for PD patients, respectively) (Table 85).

In contrast to the pattern of inpatient medical costs for dialysis patients, the leading medical cost for outpatients on dialysis was direct expenses related to dialysis treatment, accounting for $64.81 \%$ of the overall medical costs $(65.20 \%$ for HD patients and $62.25 \%$ for PD patients, respectively) (Table 86). In addition, the proportion of out-of-pocket costs was much lower than that of hospitalized patients (18.41\% vs. $29.85 \%$ ) (Tables 85 and 86 ), which might be due to the highreimbursement policy for dialysis cost.

Table 83 | Overall costs of dialysis patients, by modality

\begin{tabular}{|c|c|c|c|}
\hline & HD & PD & Total \\
\hline \multicolumn{4}{|l|}{ Sex (\%) } \\
\hline Male & $59.17 \%$ & $51.82 \%$ & $58.14 \%$ \\
\hline Female & $40.83 \%$ & $48.18 \%$ & $41.86 \%$ \\
\hline Laboratory examinations & $4.53 \%$ & $6.07 \%$ & $4.75 \%$ \\
\hline Other examinations & $2.91 \%$ & $1.74 \%$ & $2.75 \%$ \\
\hline Drugs & $25.97 \%$ & $31.07 \%$ & $26.69 \%$ \\
\hline \multicolumn{4}{|l|}{ Pattern of payment (\%) } \\
\hline UBMI paid & $76.79 \%$ & $75.48 \%$ & $76.61 \%$ \\
\hline Out-of-pocket & $23.21 \%$ & $24.52 \%$ & $23.39 \%$ \\
\hline \multicolumn{4}{|l|}{ Hospital grade (\%) } \\
\hline Tertiary hospital & $54.80 \%$ & $65.30 \%$ & $56.28 \%$ \\
\hline Secondary hospital & $32.02 \%$ & $11.33 \%$ & $29.10 \%$ \\
\hline
\end{tabular}

$\mathrm{HD}$, hemodialysis; PD, peritoneal dialysis; RMB, Renminbi; UBMI, urban basic medical insurance.

Table 84 | Costs of dialysis patients per patient, by modality

\begin{tabular}{llll}
\hline & \multicolumn{1}{c}{ HD } & \multicolumn{1}{c}{ PD } & Total \\
\cline { 2 - 3 } RMB PPPY & \multicolumn{1}{c}{ Median (IQR) } & \multicolumn{1}{c}{ Median (IQR) } & Median (IQR) \\
\hline Outpatient & $67,298(57,242-96,435)$ & $52,321(25,440-68,192)$ & $65,808(52,655-91,438)$ \\
Inpatient & $21,336(10,950-46,462)$ & $42,902(18,498-95,610)$ & $24,800(11,994-53,972)$ \\
Overall & $87,125(66,523-122,318)$ & $73,266(47,968-11,6782)$ & $85,295(63,918-121,240)$ \\
\hline
\end{tabular}

$\mathrm{HD}$, hemodialysis; IQR, interquartile range; PD, peritoneal dialysis; PPPY, per person per year; RMB, Renminbi. 
Table 85 | Inpatient costs of dialysis patients, by modality

\begin{tabular}{|c|c|c|c|}
\hline & HD & PD & Total \\
\hline Inpatient costs: RMB & $158,229,883$ & $28,964,210$ & $187,194,093$ \\
\hline Inpatient/Overall: (\%) & $42.88 \%$ & $47.88 \%$ & $43.58 \%$ \\
\hline \multicolumn{4}{|l|}{ Sex (\%) } \\
\hline Male & $58.54 \%$ & $50.56 \%$ & $57.31 \%$ \\
\hline Female & $41.46 \%$ & $49.44 \%$ & $42.69 \%$ \\
\hline \multicolumn{4}{|l|}{ Breakdown of costs (\%) } \\
\hline Laboratory examinations & $9.05 \%$ & $10.45 \%$ & $9.27 \%$ \\
\hline Other examinations & $4.23 \%$ & $2.86 \%$ & $4.02 \%$ \\
\hline Drugs & $33.76 \%$ & $34.45 \%$ & $33.87 \%$ \\
\hline Direct costs of dialysis & $21.55 \%$ & $21.02 \%$ & $21.47 \%$ \\
\hline Others & $31.40 \%$ & $31.23 \%$ & $31.37 \%$ \\
\hline \multicolumn{4}{|l|}{ Pattern of payment (\%) } \\
\hline UBMI paid & $70.47 \%$ & $68.42 \%$ & $70.15 \%$ \\
\hline Out-of-pocket & $29.53 \%$ & $31.58 \%$ & $29.85 \%$ \\
\hline \multicolumn{4}{|l|}{ Hospital grade (\%) } \\
\hline Tertiary hospital & $50.69 \%$ & $71.44 \%$ & $53.90 \%$ \\
\hline Secondary hospital & $25.05 \%$ & $10.70 \%$ & $22.83 \%$ \\
\hline Primary hospital & $24.26 \%$ & $17.85 \%$ & $23.27 \%$ \\
\hline
\end{tabular}

$\mathrm{HD}$, hemodialysis; PD, peritoneal dialysis; RMB, Renminbi; UBMI, urban basic medical insurance.

Table 86 | Outpatient costs of dialysis patients, by treatment modality

\begin{tabular}{|c|c|c|c|}
\hline & HD & PD & Total \\
\hline Outpatient costs: RMB & $210,803,235$ & $31,529,984$ & $242,333,219$ \\
\hline Outpatient/overall (\%) & $57.12 \%$ & $52.12 \%$ & $56.42 \%$ \\
\hline \multicolumn{4}{|l|}{ Sex (\%) } \\
\hline Female & $40.36 \%$ & $47.03 \%$ & $41.22 \%$ \\
\hline \multicolumn{4}{|l|}{ Breakdown of costs (\%) } \\
\hline Laboratory examinations & $1.14 \%$ & $2.06 \%$ & $1.26 \%$ \\
\hline Direct costs of dialysis & $65.20 \%$ & $62.25 \%$ & $64.81 \%$ \\
\hline Others & $11.62 \%$ & $7.01 \%$ & $11.02 \%$ \\
\hline \multicolumn{4}{|l|}{ Pattern of payment (\%) } \\
\hline UBMI paid & $81.53 \%$ & $81.97 \%$ & $81.59 \%$ \\
\hline Out-of-pocket & $18.47 \%$ & $18.03 \%$ & $18.41 \%$ \\
\hline \multicolumn{4}{|l|}{ Hospital grade (\%) } \\
\hline
\end{tabular}

$\mathrm{HD}$, hemodialysis; PD, peritoneal dialysis; RMB, Renminbi; UBMI, urban basic medical insurance. 


\title{
Chapter 12: Kidney transplant waiting list
}

\author{
Chao Yang ${ }^{1}$, Xiangxiang $\mathrm{He}^{2}$, Ying $\mathrm{Shi}^{2}$, Zhiye $\mathrm{Zhou}^{2}$, Fang Wang ${ }^{1}$ and Haibo Wang ${ }^{2,3}$ \\ ${ }^{1}$ Renal Division, Department of Medicine, Peking University First Hospital; Peking University Institute of Nephrology, Beijing, China; \\ ${ }^{2}$ China Standard Medical Information Research Center, Shenzhen, China; and ${ }^{3}$ Center for Data Science in Health and Medicine, \\ Peking University, Beijing, China
}

Kidney transplantation is one alternative renal replacement therapy of choice for patients with ESKD. As of September 1, 2013, it became mandatory to allocate organs through the China Organ Transplant Response System (COTRS), which is a national open and transparent organ allocation computer system. This chapter focuses on patients on the waiting list for kidney transplantation in China in 2015.

From January 1, 2015, to December 31, 2015, there were 25,472 candidates on the waiting list, among which 9259 candidates were newly added during this period (Figure 63 and Table 87$)$. There was a male preponderance $(67.58 \%)$, and nearly two-thirds of candidates were aged 18 to 45 years (Figures 64 and 65, and Tables 88 and 89). The top 3 regions in terms of number of candidates per million population were Shanghai, Beijing, and Tianjin (Figure 66 and Table 90). The top 3 causes of chronic kidney failure among those on the

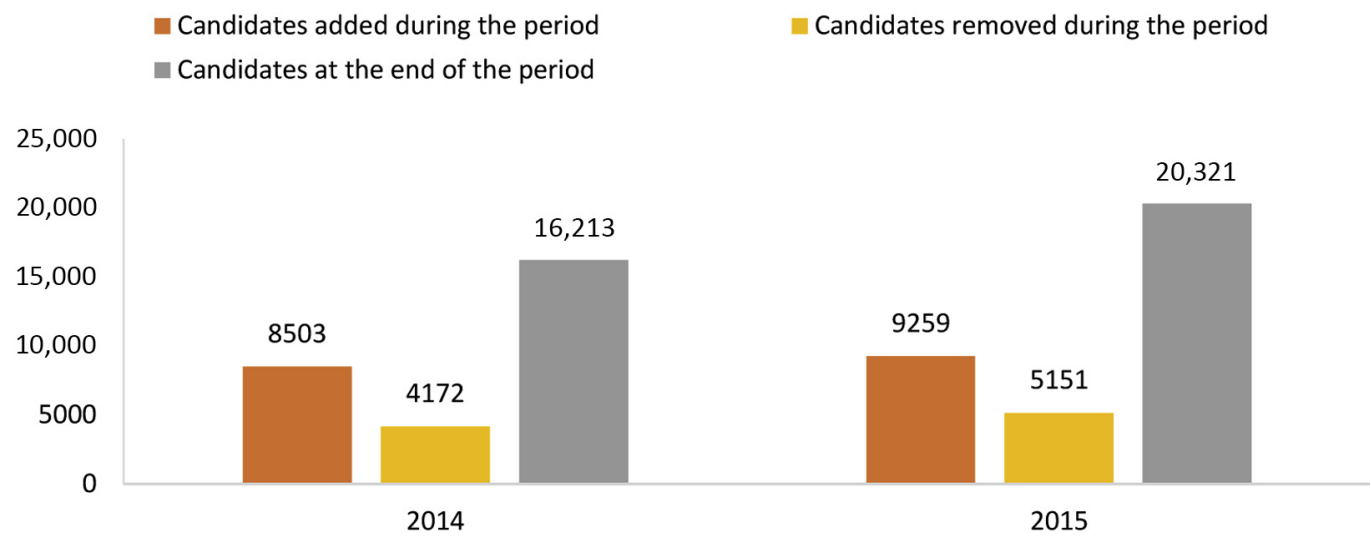

Figure 63 | Number of candidates.

Table 87| Number of candidates

\begin{tabular}{ll}
\hline January 1 to December 31,2015 & No. \\
\hline Candidates added during the period & 9259 \\
Candidates removed during the period & 5151 \\
Candidates at the end of the period & 20,321 \\
Candidates during the period & 25,472 \\
\hline
\end{tabular}

Candidates with current listings at more than one center are counted once, from the time of earliest listing to the time of latest removal. Patients listed for multi-organ

transplants are included.

waiting list were primary glomerulonephritis, secondary glomerulonephritis, and renal insufficiency of unknown cause, which accounted for $83.80 \%, 6.02 \%$, and $5.80 \%$, respectively (Figure 67 and Table 91). The majority (94.05\%) of candidates were already treated by dialysis, and $79.35 \%$ of them received HD (Figure 68 and Table 92). Altogether, 5151 candidates were removed from the waiting list during this time period; of these, $88.71 \%$ received a kidney transplant (Figure 69 and Table 93). Among the 20,321 active candidates on the waiting list on December 31, 2015, the median waiting time was 17.53 (IQR: 6.45-27.89) months (Figure 70 and Table 94).

\section{ACKNOWLEDGMENTS}

We thank Jiefu Huang of the China Organ Transplantation Development Foundation for constructive comments on this chapter.

\section{Candidates removed during the period}




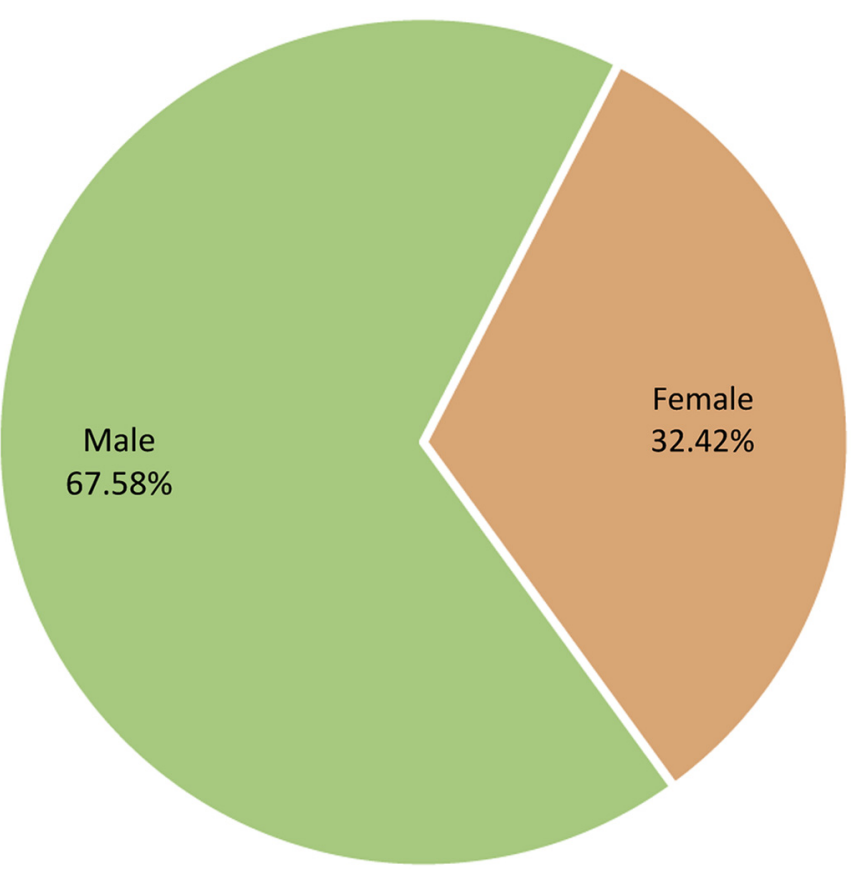

Figure 64 | Candidates, stratified by sex in 2015.

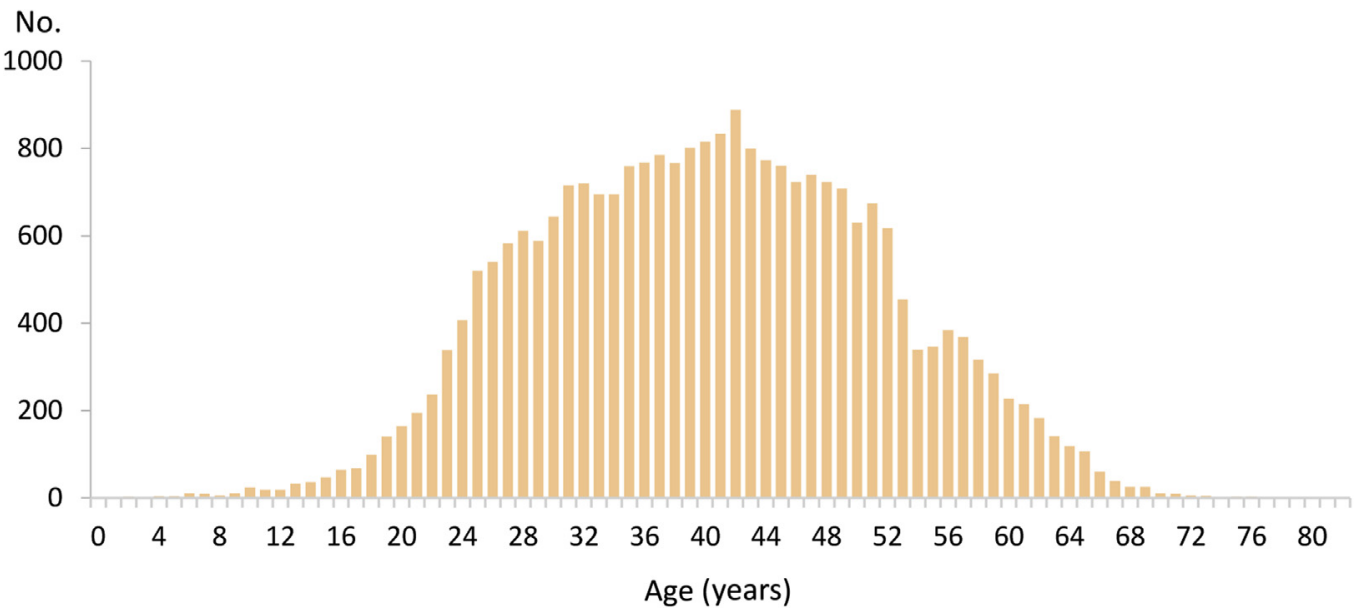

Figure 65 | Candidates, stratified by age in 2015.

Table 88 | Candidates, stratified by sex in 2015

\begin{tabular}{lcr}
\hline Sex & No. & $\%$ \\
\hline Male & 17,213 & 67.58 \\
Female & 8259 & 32.42 \\
Total & 25,472 & 100 \\
\hline
\end{tabular}


Table 89| Candidates, stratified by age in 2015

\begin{tabular}{lrr}
\hline Age (years) & $N$ & $\%$ \\
\hline$[0,18)$ & 355 & 1.39 \\
{$[18,25)$} & 1577 & 6.19 \\
{$[25,30)$} & 2840 & 11.15 \\
{$[30,35)$} & 3469 & 13.62 \\
{$[35,40)$} & 3878 & 15.22 \\
{$[40,45)$} & 4108 & 16.13 \\
{$[45,50)$} & 3654 & 14.35 \\
{$[50,55)$} & 2714 & 10.65 \\
{$[55,60)$} & 1699 & 6.67 \\
{$[60,65)$} & 882 & 3.46 \\
{$[65,70)$} & 255 & 1.00 \\
{$[70,75)$} & 32 & 7 \\
{$[75,80)$} & 2 & 0.13 \\
{$[80,85)$} & 0 & 0.03 \\
{$[85,+)$} & 25,472 & 0.01 \\
Total & & 100 \\
\hline
\end{tabular}

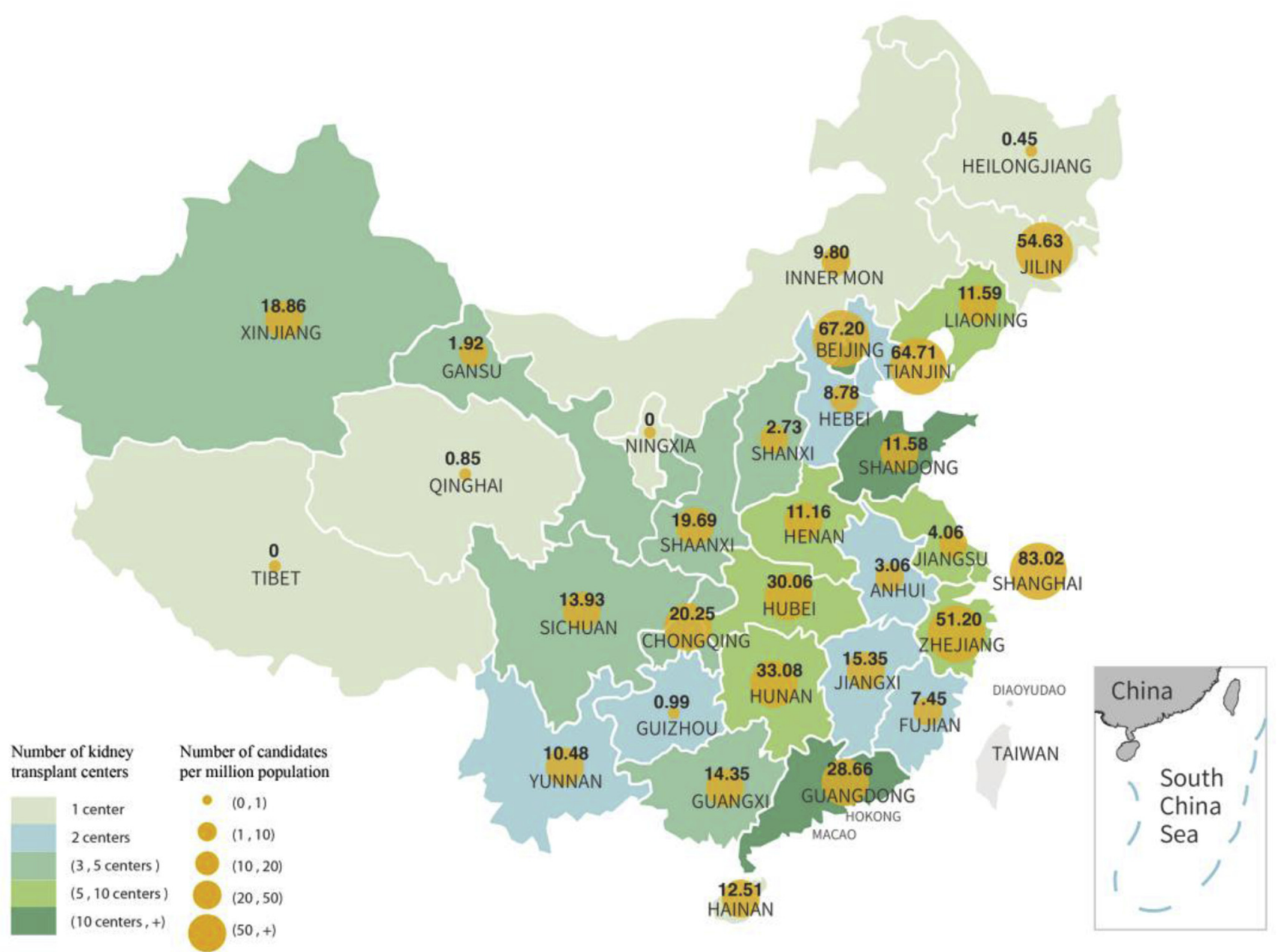

Figure 66 | Geographic distribution of candidate location in 2015. 
Table 90| Geographic distribution of candidate location in 2015

\begin{tabular}{|c|c|c|c|c|}
\hline Candidate location & No. of kidney transplant center & No. of candidates & $\%$ of total candidates & Rank of candidates \\
\hline N-Beijing & 14 & 1459 & 6.00 & 7 \\
\hline N-Tianjin & 2 & 1001 & 3.93 & 11 \\
\hline N-Hebei & 2 & 652 & 2.56 & 15 \\
\hline N-Shanxi & 3 & 100 & 0.39 & 25 \\
\hline N-Inner Mongolia & 1 & 246 & 0.97 & 22 \\
\hline NE-Liaoning & 5 & 508 & 1.99 & 17 \\
\hline NE-Jilin & 1 & 1504 & 5.90 & 6 \\
\hline NE-Heilongjiang & 1 & 17 & 0.07 & 28 \\
\hline E-Shanghai & 7 & 2005 & 7.87 & 4 \\
\hline E-Jiangsu & 5 & 324 & 1.27 & 20 \\
\hline E-Zhejiang & 6 & 2836 & 11.13 & 2 \\
\hline E-Anhui & 2 & 188 & 0.74 & 23 \\
\hline E-Fujian & 2 & 286 & 1.12 & 21 \\
\hline E-Jiangxi & 2 & 701 & 2.75 & 13 \\
\hline E-Shandong & 11 & 1140 & 4.48 & 9 \\
\hline C-Henan & 6 & 1058 & 4.15 & 10 \\
\hline C-Hubei & 7 & 1759 & 6.91 & 5 \\
\hline C-Hunan & 8 & 2244 & 8.81 & 3 \\
\hline S-Guangdong & 13 & 3109 & 12.21 & 1 \\
\hline S-Guangxi & 4 & 688 & 2.70 & 14 \\
\hline S-Hainan & 1 & 114 & 0.45 & 24 \\
\hline SW-Chongqing & 4 & 611 & 2.40 & 16 \\
\hline SW-Sichuan & 4 & 1143 & 4.49 & 8 \\
\hline SW-Guizhou & 2 & 35 & 0.14 & 27 \\
\hline SW-Yunnan & 2 & 497 & 1.95 & 18 \\
\hline SW-Tibet & 1 & & & \\
\hline NW-Shaannxi & 4 & 747 & 2.93 & 12 \\
\hline NW-Gansu & 3 & 50 & 0.20 & 26 \\
\hline NW-Qinghai & 1 & 5 & 0.02 & 29 \\
\hline NW-Ningxia & 1 & & & \\
\hline NW-Xinjiang & 4 & 445 & 1.75 & 19 \\
\hline China & 129 & 25,472 & - & - \\
\hline
\end{tabular}

C, Central of China; E, East of China; N, North of China; NE, Northeast of China; S, South of China; SW, Southwest of China; NW, Northwest of China.

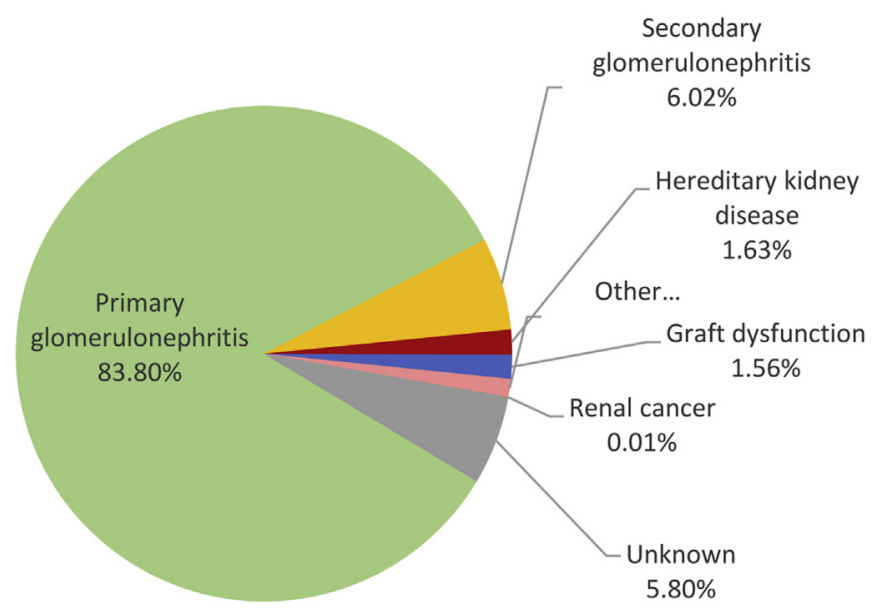

Figure 67| Candidates, stratified by causes in 2015. 
Table 91 | Candidates, stratified by causes in 2015

\begin{tabular}{lrr}
\hline Diagnosis & $N$ & N \\
\hline Primary glomerulonephritis & 21,346 & 1534 \\
Secondary glomerulonephritis & 415 & 3.80 \\
Hereditary kidney disease & 397 & 301 \\
Graft dysfunction & 2 & 1.63 \\
Other & 1.56 \\
Renal cancer & 1.18 \\
Unknown & 0.01 \\
Total & 25,472 \\
\hline
\end{tabular}

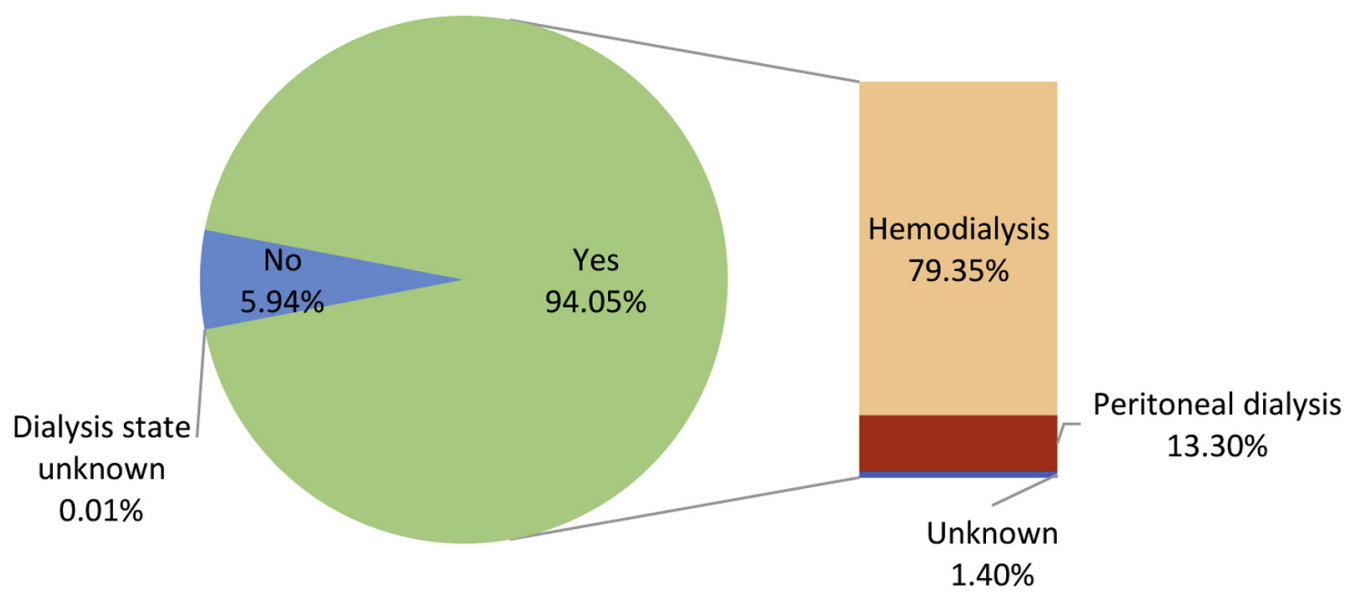

Figure 68 | Candidates, stratified by dialysis state in 2015.

Table 92 | Candidates, stratified by dialysis state in 2015

\begin{tabular}{lrr}
\hline Dialysis state & $\boldsymbol{N}$ & $\%$ \\
\hline Yes & 23,956 & 94.05 \\
Hemodialysis & 20,211 & 79.35 \\
Peritoneal dialysis & 3389 & 13.30 \\
Unknown & 356 & 1.40 \\
No & 1514 & 5.94 \\
Unknown & 2 & 0.01 \\
Total & 25,472 & 100 \\
\hline
\end{tabular}

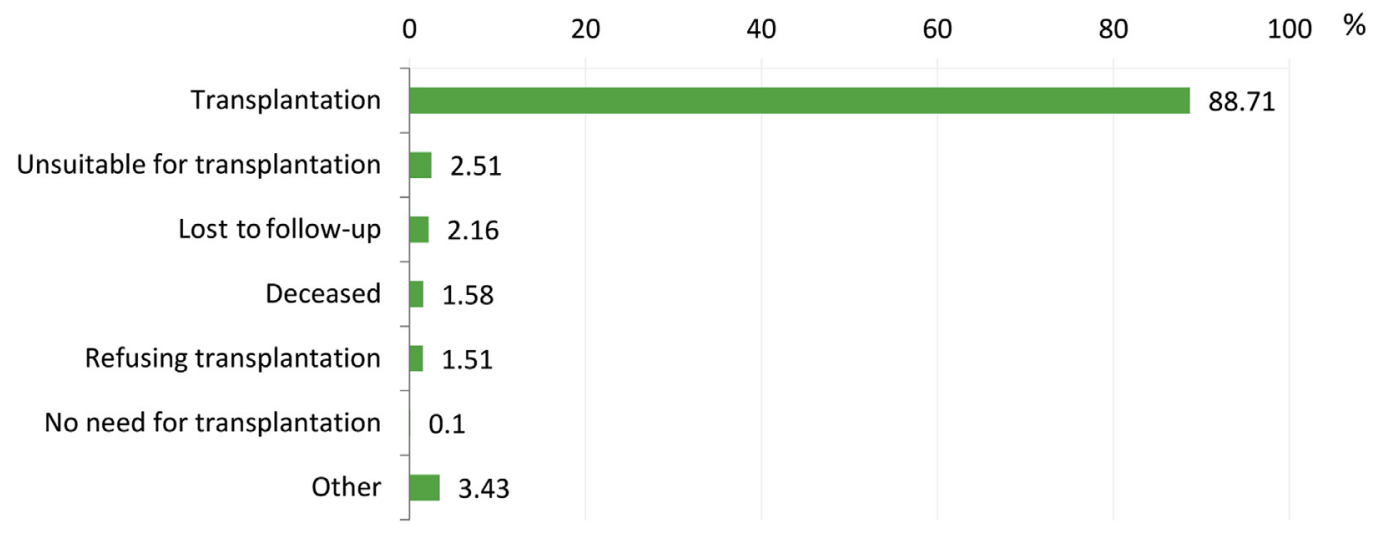

Figure 69| Candidates, stratified by cause of removal in 2015. 
Table 93 | Candidates, stratified by cause of removal in 2015

\begin{tabular}{lrr}
\hline Cause of removal & $N$ & \% \\
\hline Transplantation & 4520 & 124 \\
Unsuitable for transplantation & 81 & 2.51 \\
Lost to follow-up & 136 & 2.16 \\
Deceased & 177 & 1.58 \\
Refusing transplantation & 88 & 1.51 \\
No need for transplantation & 25 & 0.10 \\
Other & 5151 & 3.43 \\
Total (candidates removed during the period) & & 100 \\
\hline
\end{tabular}

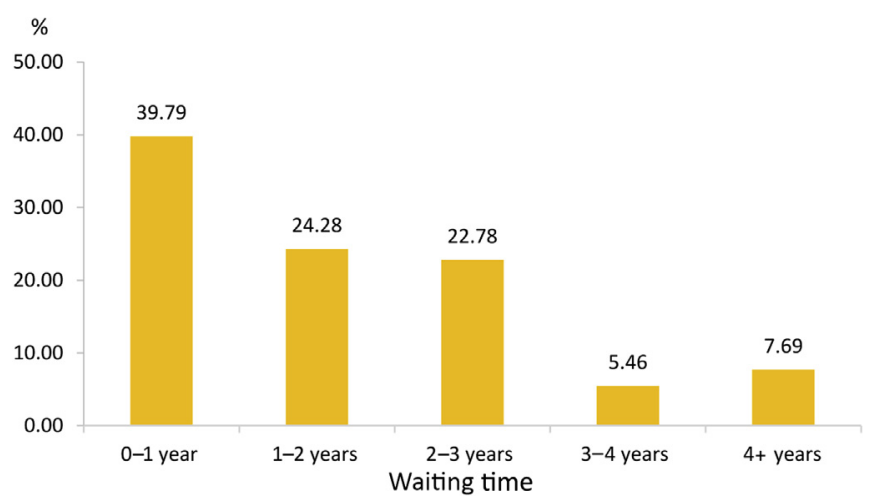

Figure 70| Active candidates, stratified by waiting time in 2015.

Table 94 | Active candidates, stratified by waiting time

\begin{tabular}{lrr}
\hline Waiting time & $N$ & $\%$ \\
\hline$[0,1$ year) & 8085 & 39.79 \\
{$[1,2$ years) } & 4934 & 24.28 \\
{$[2,3$ years) } & 4630 & 22.78 \\
{$[3,4$ years) } & 1110 & 5.46 \\
{$[4$ years, +$)$} & 1562 & 7.69 \\
Total (candidates at the end of the period) & 20,321 & 100 \\
\hline
\end{tabular}

Among the 20,321 active candidates at the end of 2015 , the median waiting time was $17.53(6.45,27.89)$ months. 


\section{Chapter 13: Discussion}

Based on a general population-based survey performed from 2009 to 2010, it is reported that the prevalence of CKD is $10.8 \%,{ }^{14}$ which is comparable to the prevalence in reports from developed countries. ${ }^{15}$ In our present analyses based on hospitalized patients and discharge diagnoses, patients with CKD constituted $4.8 \%$ of 18.5 million hospitalized patients, which is substantially lower than data from the survey. In order to understand the potential reasons for the discrepancy, we have performed a validation study using data of 67,376 patients from 3 hospitals in the Hospital Quality Monitoring System (HQMS) database, which are the pilot hospitals that have accomplished the HQMS phase 2 data collection expansion to the data of laboratory tests. ${ }^{16}$ $\mathrm{CKD}$ was defined as the presence of proteinuria and/or estimated glomerular filtration rate $<60 \mathrm{ml} / \mathrm{min}$ per 1.73 $\mathrm{m}^{2}$. The sensitivity and specificity of CKD identification by discharge diagnoses were $34.2 \%$ and $97.8 \%$, respectively, which could partly explain the low percentage of CKD among hospitalized patients. Actually the percentage of CKD in our results comprehensively reflects the prevalence, hospitalization rate, and diagnostic rate, which should be noted when interpreting results from Section I.

Regarding the spectrum of CKD, our results as well as previous reports from our group ${ }^{16,17}$ have revealed increasing trends of DKD, HTN, and ON, mostly accompanied by kidney stone. However, population-based studies regarding the prevalence of types of kidney disease in China are extremely limited. For example, despite DKD being an important complication of diabetes, there are few epidemiological studies regarding diabetes including markers of kidney damage in China. In the referred cohort of type 2 diabetic patients, Asians had a higher prevalence of elevated urinary albumin-to-creatinine ratio compared with nonAsians. ${ }^{18}$ However, Asians included heterogeneous ethnicity, and information from referred patients may be biased. In our recent analyses using data of 8811 community-based patients with diabetes, the percentage of isolated estimated glomerular filtration rate $<60 \mathrm{ml} / \mathrm{min}$ per $1.73 \mathrm{~m}^{2}$, isolated proteinuria, and proteinuria combined with estimated glomerular filtration rate $<60 \mathrm{ml} / \mathrm{min}$ per $1.73 \mathrm{~m}^{2}$ was $10.7 \%$, $17.5 \%$, and $4.6 \%$, respectively (accepted by Journal of Diabetes and Its Complications). Those numbers were comparatively lower compared with results from the UK Prospective Diabetes Study (UKPDS) involving 4006 patients with diabetes, which was $14.4 \%, 24.4 \%$ and $13.9 \%$, respectively. ${ }^{19}$
Regarding other types of CKD, IgA nephropathy is thought to be the major pathological type of primary glomerulonephritis in East and Pacific Asian countries. According to a recent study, ${ }^{20}$ the incidence was 45 cases per million population per year in Japan compared with 31 cases per million population per year in France. A recent study from our group utilizing the same inpatient dataset revealed that a decreasing trend of IgA nephropathy was observed (from 19.0\% in 2010 to $10.6 \%$ in 2015 among patients with primary glomerulonephritis), ${ }^{21}$ while the incidence rate could not be calculated based on that dataset. Although cystic kidney disease is a relatively common cause of CKD in Western countries, data from regional dialysis registry in Beijing and Shanghai indicated that cystic kidney disease accounted for less than 3\% of dialysis patients. ${ }^{22,23}$

Hypertension contributes to increased morbidity and mortality in the CKD population, and therefore is an important component of managing patients of CKD. According to a recent study based on a multicenter prospective cohort of patients with CKD in China, the awareness, treatment, and control rates of hypertension were $80.7 \%, 95.6 \%$, and $57.1 \%$, respectively. ${ }^{24}$ The treatment and control rates were improved compared with results from a study in 2004 to 2005 (85.9\% and $30.0 \%$, respectively), ${ }^{25}$ while the control rate was still lower compared with results from Chronic Renal Insufficiency Cohort in the US $(67.1 \%) .^{26}$

In conclusion, by integrating and mining national patientlevel administrative or claims databases from various sources, as well as carefully interpreting results based on understanding the nature of those databases, we have been able to provide a comprehensive description of the burden of CKD and ESKD in China. Besides providing evidence for setting public health policy, these results should also inspire the direction of future research.

\section{ACKNOWLEDGMENTS}

We thank the National Health Commission of China, China Health Insurance Research Association, China Organ Transplantation Development Foundation, Peking University, and China Standard Medical Information Research Center for the support of this study. We thank CK-NET collaborating centers, members, and volunteers for their hard work and efforts. We thank every participant who has contributed important and invaluable data to CK-NET. 


\section{References}

1. Zhang L, Wang H, Long J, et al. China Kidney Disease Network (CK-NET) 2014 Annual Data Report. Am J Kidney Dis. 2016;69(Suppl 2):S1-S149.

2. GBD 2013 Mortality and Causes of Death Collaborators. Global, regional, and national age-sex specific all-cause and cause-specific mortality for 240 causes of death, 1990-2013: a systematic analysis for the Global Burden of Disease Study 2013. Lancet. 2015;385:117-171.

3. Fried LF, Emanuele N, Zhang JH, et al. Combined angiotensin inhibition for the treatment of diabetic nephropathy. N Engl J Med. 2013;369:1892-1903.

4. de Boer IH, Rue TC, Hall YN, et al. Temporal trends in the prevalence of diabetic kidney disease in the United States. JAMA. 2011;305:2532-2539.

5. Saran R, Robinson B, Abbott KC, et al. US Renal Data System 2017 Annual Data Report: Epidemiology of Kidney Disease in the United States. Am J Kidney Dis. 2018;71:A7.

6. Hanafusa N, Nakai S, Iseki K, Tsubakihara Y. Japanese society for dialysis therapy renal data registry-a window through which we can view the details of Japanese dialysis population. Kidney Int Suppl (2011). 2015;5: $15-22$.

7. Zhang L, Zuo L. Current burden of end-stage kidney disease and its future trend in China. Clin Nephrol. 2016;86:27-28.

8. Ho YW, Chau KF, Choy BY, et al. Hong Kong Renal Registry Report 2012. Hong Kong J Nephrol. 2013;15:28-43.

9. Yang WC, Hwang SJ. Incidence, prevalence and mortality trends of dialysis end-stage renal disease in Taiwan from 1990 to 2001: the impact of national health insurance. Nephrol Dial Transplant. 2008;23:3977-3982.

10. Kidney Disease: Improving Global Outcomes (KDIGO) CKD-MBD Update Work Group. KDIGO 2017 Clinical Practice Guideline Update for the Diagnosis, Evaluation, Prevention, and Treatment of Chronic Kidney Disease-Mineral and Bone Disorder (CKD-MBD). Kidney Int Suppl. 2017;7:1-59.

11. Kidney Disease: Improving Global Outcomes (KDIGO) Anemia Work Group. KDIGO Clinical Practice Guideline for Anemia in Chronic Kidney Disease. Kidney Int Suppl. 2012;2:279-335.

12. Daratha KB, Short RA, Corbett CF, et al. Risks of subsequent hospitalization and death in patients with kidney disease. Clin J Am Soc Nephrol. 2012;7:409416.

13. United States Renal Data System. 2017 USRDS Annual Data Report: Epidemiology of kidney disease in the United States. Bethesda, MD: National
Institutes of Health, National Institute of Diabetes and Digestive and Kidney Diseases; 2017.

14. Zhang L, Wang F, Wang L, et al. Prevalence of chronic kidney disease in China: a cross-sectional survey. Lancet. 2012;379:815-822.

15. United States Renal Data System. 2017 USRDS Annual Data Report: Epidemiology of kidney disease in the United States. Bethesda, MD: National Institutes of Health, National Institute of Diabetes and Digestive and Kidney Diseases; 2017.

16. Huang $\mathrm{Y}, \mathrm{Xu} \mathrm{D}$, Long J, et al. The spectrum of chronic kidney disease in China: a national study based on hospitalized patients from 2010 to 2015 [e-pub ahead of print]. Nephrology. https://doi.org/10.1111/nep.13489.

17. Zhang L, Long J, Jiang W, et al. Trends in chronic kidney disease in China. N Engl J Med. 2016;375:905-906.

18. Parving $\mathrm{HH}$, Lewis JB, Ravid $\mathrm{M}$, et al. Prevalence and risk factors for microalbuminuria in a referred cohort of type II diabetic patients: a global perspective. Kidney Int. 2006;69:2057-2063.

19. Retnakaran R, Cull CA, Thorne KI, et al. Risk factors for renal dysfunction in type 2 diabetes: U.K. Prospective Diabetes Study 74. Diabetes. 2006;55:1832-1839.

20. Schena FP, Nistor I. Epidemiology of IgA nephropathy: a global perspective. Semin Nephrol. 2018;38:435-442.

21. Li J, Cui Z, Long J, et al. Primary glomerular nephropathy among hospitalized patients in a national database in China. Nephrol Dial Transplant. 2018;33:2173-2181.

22. Beijing Hemodialysis Quality Control and Improvement Center. The Annual Data Report of Beijing Hemodialysis Registry, 2012. Chin J Blood Purif. 2012;z1:42.

23. Shanghai Kidney Disease Quality Control Center. Shanghai Renal Registry Annual Data Report 2017. Available at: http://www.cnrds.org. Accesed October 29, 2018.

24. Yan Z, Wang Y, Li S, et al. Hypertension control in adults with CKD in China: baseline results from the Chinese Cohort Study of Chronic Kidney Disease (C-STRIDE). Am J Hypertens. 2018;31:486-494.

25. Wang $\mathrm{Y}$, Zhang L, Li X, et al. Improvement of awareness, treatment and control of hypertension among chronic kidney disease patients in China from 1999 to 2005. Hypertens Res. 2009;32:444-449.

26. Muntner P, Anderson A, Charleston J, et al. Hypertension awareness treatment, and control in adults with CKD: results from the Chronic Renal Insufficiency Cohort (CRIC) Study. Am J Kidney Dis. 2010;55:415-418. 


\section{Appendices: Definitions of ICD coding}

\section{Appendix 1 | Coding of various CKD etiologies}

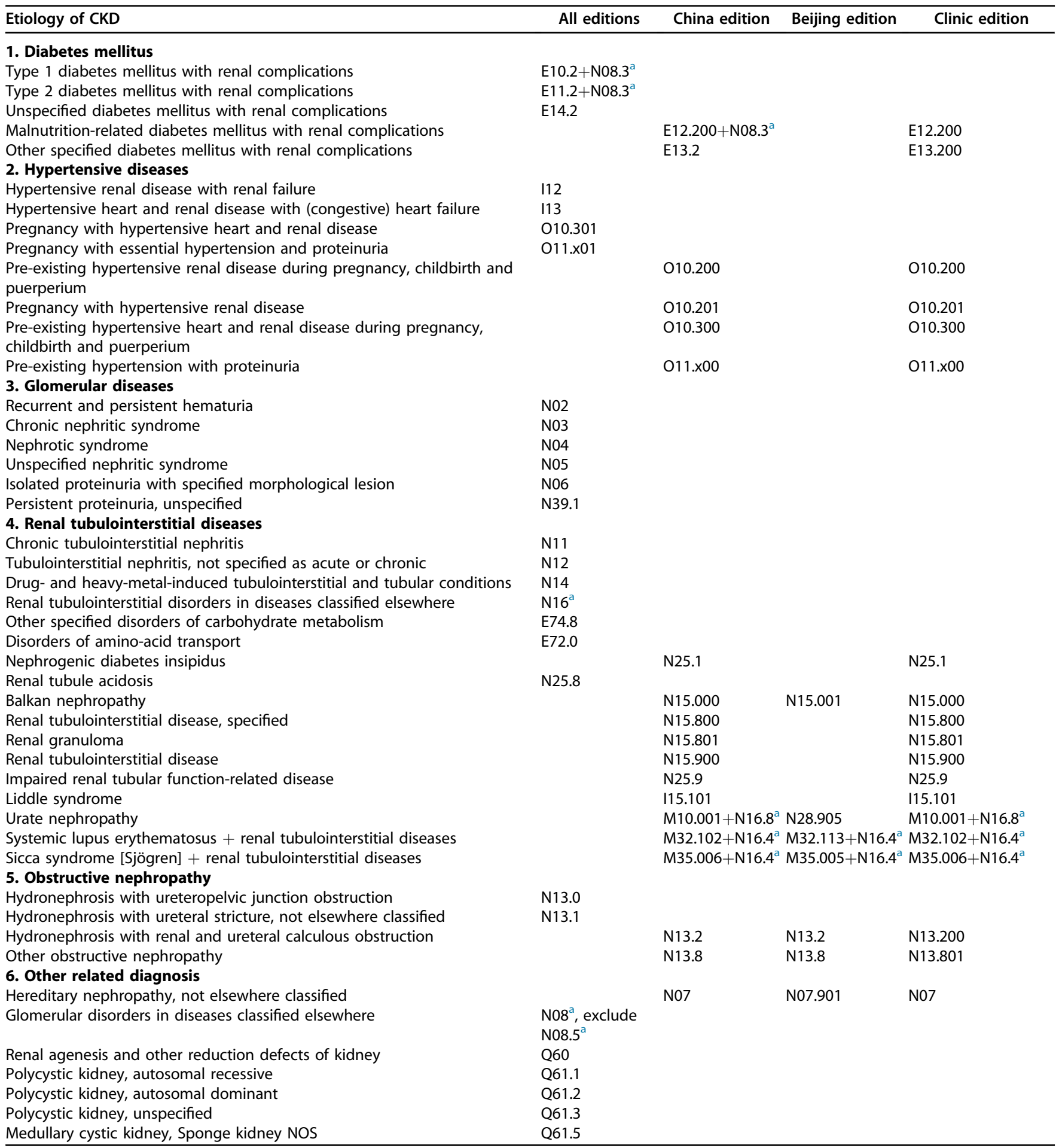




\section{Appendix 1 | (Continued) Coding of various CKD etiologies}

\begin{tabular}{|c|c|c|c|c|}
\hline Etiology of CKD & All editions & China edition & Beijing edition & Clinic edition \\
\hline Lobulated, fused and horseshoe kidney & Q63.1 & & & \\
\hline Congenital malformation of kidney, unspecified & Q63.9 & & & \\
\hline Gout due to impairment of renal function & & M10.300 & M10.393 & M10.300 \\
\hline Unspecified contracted kidney & N26 & & & \\
\hline Ischemia and infarction of kidney & N28.0 & & & \\
\hline Other specified disorders of kidney and ureter & N28.8 & & & \\
\hline Disorders of kidney and ureter, unspecified & N28.9 & & & \\
\hline Congenital renal failure & & P96.0 & P96.0 & P96.000 \\
\hline Extrarenal uremia & R39.2 & & & \\
\hline Aortic arch syndrome + renovascular hypertension & & $M 31.4+115.0$ & $177.604+115.0$ & $177.600 \times 004+115.0$ \\
\hline Goodpasture syndrome & M31.001 & & & \\
\hline Renal osteodystrophy & N25.0 & & & \\
\hline Failure and rejection of renal transplantation & T86.1 & & & \\
\hline Hemolytic uremic syndrome & D59.3 & & & \\
\hline Dialysis & Z49 & & & \\
\hline Renal allergic purpura & $\mathrm{D} 69.005+\mathrm{N} 08.2^{\mathrm{a}}$ & & & \\
\hline Lupus nephritis & & $\mathrm{M} 32.101+\mathrm{N} 08.5^{\mathrm{a}}$ & $\mathrm{M} 32.105+\mathrm{N} 08.5^{\mathrm{a}}$ & $\mathrm{M} 32.101+\mathrm{N} 08.5^{\mathrm{a}}$ \\
\hline Goodpasture syndrome-related glomerulonephritis & & $\mathrm{M} 31.003+\mathrm{N} 08.5^{\mathrm{a}}$ & & $\mathrm{M} 31.003+\mathrm{N} 08.5^{\mathrm{a}}$ \\
\hline Antiglomerular basement membrane antibody-related disease & & $\mathrm{M} 31.002+\mathrm{N} 08.5^{\mathrm{a}}$ & $\mathrm{M} 31.005+\mathrm{N} 08.5^{\mathrm{a}}$ & $\mathrm{M} 31.002+\mathrm{N} 08.5^{\mathrm{a}}$ \\
\hline Microscopic polyangiitis & & M31.700 & M31.701 & M31.700 \\
\hline ANCA-related nephritis & & $\mathrm{M} 31.701+\mathrm{N} 08.5^{\mathrm{a}}$ & M31.802 & $\mathrm{M} 31.701+\mathrm{N} 08.5^{\mathrm{a}}$ \\
\hline Thrombotic thrombocytopenic purpura-related glomerulonephritis & & $\mathrm{M} 31.102+\mathrm{N} 08.5^{\mathrm{a}}$ & & $\mathrm{M} 31.102+\mathrm{N} 08.5^{\mathrm{a}}$ \\
\hline Wegener's granulomatosis-related glomerulonephritis & & $\mathrm{M} 31.303+\mathrm{N} 08.5^{\mathrm{a}}$ & & $\mathrm{M} 31.303+\mathrm{N} 08.5^{\mathrm{a}}$ \\
\hline Pregnancy with nephrotic syndrome & & O26.801 & O26.811 & O26.801 \\
\hline Pregnancy with glomerulonephritis & & O26.804 & O26.812 & O26.804 \\
\hline Pregnancy with renal failure & & O26.802 & $\mathrm{O} 26.813$ & O26.802 \\
\hline HBV-related nephritis & & $\mathrm{B} 18.103+\mathrm{N} 08.0^{\mathrm{a}}$ & B18.102 & $\mathrm{B} 18.103+\mathrm{N} 08.0^{\mathrm{a}}$ \\
\hline HCV-related nephritis & & $\mathrm{B} 18.205+\mathrm{N} 08.0^{\mathrm{a}}$ & B18.208 & $\mathrm{B} 18.205+\mathrm{N} 08.0^{\mathrm{a}}$ \\
\hline Cryoglobulinemia-related glomerulonephritis & & $\mathrm{D} 89.101+\mathrm{N} 08.2^{\mathrm{a}}$ & & $\mathrm{D} 89.101+\mathrm{N} 08.2^{\mathrm{a}}$ \\
\hline Hereditary amyloidosis nephropathy & & E85.002 & E85.003 & E85.002 \\
\hline Amyloidosis-related nephropathy & & $\mathrm{E} 85.411+\mathrm{N} 29.8^{\mathrm{a}}$ & E85.410+N08.4 & $\mathrm{E} 85.411+\mathrm{N} 29.8^{\mathrm{a}}$ \\
\hline Psoriatic nephritis & & $\mathrm{L} 40.803+$ & $\mathrm{L} 40.802+\mathrm{N} 05.9^{\mathrm{a}}$ & $\mathrm{L} 40.800 \times 002+\mathrm{N} 05.9^{\mathrm{a}}$ \\
\hline Kidney injury-related gout & & M10.300 & M10.393 & M10.300 \\
\hline Syphilitic nephritis & & & $\mathrm{A} 52.712+\mathrm{N} 08.0^{\mathrm{a}}$ & $\mathrm{A} 52.700 \times 012+\mathrm{N} 08.0^{\mathrm{a}}$ \\
\hline Lupus kidney injury & & & $\mathrm{M} 32.112+\mathrm{N} 08.5^{\mathrm{a}}$ & \\
\hline Lupus nephritis & & $\mathrm{M} 32.101+\mathrm{N} 08.5^{\mathrm{a}}$ & $\mathrm{M} 32.105+\mathrm{N} 08.5^{\mathrm{a}}$ & $\mathrm{M} 32.101+\mathrm{N} 08.5^{\mathrm{a}}$ \\
\hline Lupus tubulointerstitial kidney & & $\mathrm{M} 32.102+\mathrm{N} 16.4^{\mathrm{a}}$ & $\mathrm{M} 32.113+\mathrm{N} 16.4^{\mathrm{a}}$ & $\mathrm{M} 32.102+\mathrm{N} 16.4^{\mathrm{a}}$ \\
\hline Gouty nephropathy & & & M10.391 & M10.300x091 \\
\hline Gouty nephrolithiasis & & $\mathrm{M} 10.005+\mathrm{N} 22.8^{\mathrm{a}}$ & M10.392 & $\mathrm{M} 10.005+\mathrm{N} 22.8^{\mathrm{a}}$ \\
\hline
\end{tabular}

ANCA, antineutrophil cytoplasmic antibodies; CKD, chronic kidney disease; HBV, hepatitis B virus; HCV, hepatitis C virus.

${ }^{a}$ Denotes to any possible number so that the whole sequence represents all the subclasses of the disease.

Appendix 2| Coding of CKD stages

\begin{tabular}{|c|c|c|c|}
\hline Stage of CKD & China edition & Beijing edition & Clinic edition \\
\hline CKD stage 1 & N18.801 & N18.914 & N18.801 \\
\hline CKD stage 2 & N18.802 & N18.915 & N18.802 \\
\hline CKD stage 3 & N18.803 & N18.916 & N18.803 \\
\hline CKD stage 4 & N18.804 & N18.917 & N18.804 \\
\hline CKD stage 5 & N18.001 & N18.918 & N18.001 \\
\hline
\end{tabular}

CKD, chronic kidney disease. 


\section{Appendix 3| Coding of diabetes mellitus}

\begin{tabular}{ll}
\hline Etiology & ICD coding \\
\hline Type 1 diabetes mellitus & E10 \\
Type 2 diabetes mellitus & E11 \\
Malnutrition-related diabetes mellitus & E12 \\
Other specified diabetes mellitus & E13 \\
Unspecified diabetes mellitus & E14 \\
\hline
\end{tabular}

ICD, International Classification of Diseases.

\section{Appendix 4| Coding of hypertension}

\begin{tabular}{ll}
\hline Etiology & ICD coding \\
\hline Essential (primary) hypertension & 110 \\
Hypertensive heart disease & 111 \\
Hypertensive renal disease & 112 \\
Hypertensive heart and renal disease & 113 \\
Secondary hypertension & 115 \\
\hline
\end{tabular}

ICD, International Classification of Diseases.

Appendix 5| Coding of CVD

Etiology

\section{Cerebral stroke}

Subarachnoid hemorrhage

Intracerebral hemorrhage

Acute ischemic cerebral stroke

Transient ischemic attack

\section{Coronary heart disease}

Angina pectoris

Acute myocardial infarction

Subacute myocardial infarction

Complications after myocardial infarction

Other acute ischemic heart disease

Chronic ischemic heart disease

\section{Heart failure}

Whole-heart failure

Right heart failure

Right ventricular failure

Acute right heart failure

Left heart failure

Left ventricular failure

Left atrial failure

Chronic left heart insufficiency

Left heart failure with acute pulmonary edema

Congestive heart failure

Acute heart failure

Chronic heart failure

Other heart failure

Postoperative heart failure and pulmonary edema

Heart failure of newborns

Hypertensive heart failure

Hypertensive heart disease with (congestive) heart failure

All editions China edition Beijing edition Clinic edition

160

161

163

164

H34.1

G45

120

121

122

123

124

125

\begin{tabular}{lll} 
& 150.003 & 150.002 \\
150.001 & 150.004 & 150.001 \\
150.005 & & $150.000 \times 005$ \\
& & $150.000 \times 006$ \\
150.100 & 150.106 & $150.100 \times 006$ \\
& & 150.100 \\
& & 150.102 \\
& 150.103 & 150.105 \\
150.000 & 150.107 & 150.103 \\
& 150.001 & 150.000 \\
150.900 & 150.904 & 150.907 \\
& 150.905 & 150.908 \\
$P 29.000$ & 150.911 & 150.900 \\
& 197.104 & $197.100 \times 004$ \\
111.000 & $P 29.001$ & $P 29.000$ \\
& & 111.001 \\
& & 111.000 \\
\hline
\end{tabular}




\section{Appendix 5 | (Continued) Coding of CVD}

Etiology

All editions China edition Beijing edition Clinic edition

Hypertensive heart disease without (congestive) heart failure

Hypertensive heart disease and kidney disease with congestive heart failure

Hypertensive heart disease and kidney disease with congestive heart failure and renal failure

113.000

111.900

Intractable heart failure

Heart failure after cardiac surgery

Postoperative heart failure

Chronic left heart insufficiency

Cardiac insufficiency

Cardiac insufficiency of newborns

113.000

113.200

113.200

$150.900 \times 017$

197.102

197.102

197.803

150.103

197.803

150.901

150.902

150.105

Acute exacerbation of chronic cardiac insufficiency

P29.001

Acute left heart failure

Acute pulmonary edema

Pregnancy with heart failure

Pregnancy with cardiac insufficiency

Childbirth with heart failure

Pregnancy with left heart failure

Puerperal cardiac insufficiency

Acute pulmonary edema after postpartum

P29.001

Heart failure due to anesthesia during pregnancy

Heart failure due to anesthesia during childbirth

Heart failure after obstetric surgery or operation

Heart failure due to anesthesia during puerperium

Low cardiac output syndrome

Cardiac function, class I

Cardiac function, class II

Cardiac function, class III

Cardiac function, class IV

Cardiac function, class II (NYHA)

Cardiac function, class III (NYHA)

Cardiac function, class II III (NYHA)

Cardiac function, class IV(NYHA)

Circulatory failure

Pulmonary edema

Cardiogenic shock

Respiratory and circulatory failure

Cardiogenic asthma

\section{Atrial fibrillation}

Atrial fibrillation

Idiopathic atrial fibrillation

Persistent atrial fibrillation

099.417

099.429

075.403

099.402

099.507

(150.902

150.900×002

150.102

$150.900 \times 018$

$\mathrm{J} 81 \times \mathrm{x} 02$

099.408

099.429

099.423

099.434

099.508

029.102

074.202

075.402

089.102

150.901

150.902

150.901

$150.903 \quad 150.907$

150.907
150.908

$150.905 \quad 150.910$

Chronic atrial fibrillation

Pregnancy with atrial fibrillation

Atrial fibrillation with flutter

Primary atrial fibrillation

Long-term persistent atrial fibrillation

150.101

J81.x00×002

Acute atrial fibrillation

Permanent atrial fibrillation

150.104

$099.400 \times 008$

099.414

075.403

099.424

099.402

099.508

$099.500 \times 008$

$074.200 \times 002$

$089.100 \times 002$

Long-range persistent atrial fibrillation

$148 \times x 04$

$148 . \times 01$

$148 \times 02$

$148 \times 07$

$148 \times 08$

$099.427 \quad 099.427$

150.903

150.904

150.905

$150.900 \times 007$

$150.900 \times 008$

$150.900 \times 009$

$150.900 \times 010$

New diagnosis of atrial fibrillation

148.x00

148xx01

R57.901

J81.x00

R57.000

J96.900

150.104

148.x01

$148 . \times 05$

148.x00×007

148. $\times 00 \times 008$

$099.400 \times 027$

$148 . \times 00$

148.x00×009

$148 . \times 00 \times 011$

$148 . \times 00 \times 012$

$148 . \times 00 \times 013$

$148 . \times 00 \times 014$

Paroxysmal atrial fibrillation

148.x02

$148 \times x 06$

$148 . \times 00 \times 015$

CVD, cardiovascular disease; NYHA, New York Heart Association. 


\section{Appendix 6| Coding of CVD operations}

\begin{tabular}{|c|c|c|c|}
\hline Operation & China edition & Beijing edition & Clinic edition \\
\hline \multirow[t]{6}{*}{ Coronary angiography (CAG) } & & 88.55001 & $\begin{array}{l}88.5500 \\
88.5,500 \times 002\end{array}$ \\
\hline & & 88.56001 & $\begin{array}{l}88.5600 \\
88.5,600 \times 002\end{array}$ \\
\hline & & 88.57002 & 88.5701 \\
\hline & & & 88.5700 \\
\hline & & & $88.5,700 \times 003$ \\
\hline & & & 88.5900 \\
\hline \multirow[t]{6}{*}{ Percutaneous coronary intervention (PCI) } & & 36.06003 & 36.0602 \\
\hline & & & 36.0601 \\
\hline & & 36.06004 & 36.0600 \\
\hline & & 36.07003 & 36.0700 \\
\hline & & & $36.0,700 \times 004$ \\
\hline & & & 36.0701 \\
\hline \multirow[t]{8}{*}{ Coronary artery bypass grafting (CABG) } & & 36.11001 & \\
\hline & & 36.12001 & \\
\hline & & 36.13001 & \\
\hline & & 36.14001 & \\
\hline & & 36.15001 & \\
\hline & & 36.16001 & \\
\hline & & 36.17001 & \\
\hline & & 36.2001 & \\
\hline \multirow[t]{33}{*}{ Pacemaker } & Z95.000 & & Z95.000 \\
\hline & T82.700 & & T82.700 \\
\hline & T82.703 & T82.702 & T82.703 \\
\hline & T82.100 & & \\
\hline & T82.101 & & \\
\hline & T82.102 & & \\
\hline & T82.103 & & \\
\hline & T82.800 & & T82.800 \\
\hline & T82.903 & T82.801 & T82.903 \\
\hline & T82.904 & & T82.904 \\
\hline & T85.707 & & \\
\hline & Z45.007 & & \\
\hline & Z45.001 & & Z45.001 \\
\hline & Z45.002 & & \\
\hline & & Z95.001 & \\
\hline & Z45.004 & Z45.003 & Z45.004 \\
\hline & & & T82.100x002 \\
\hline & & & T82.100x003 \\
\hline & & T82.702 & T82.700x002 \\
\hline & & & Z45.000 \\
\hline & Z45.003 & & Z45.003 \\
\hline & Z45.005 & & Z45.005 \\
\hline & Z45.006 & & Z45.006 \\
\hline & & 37.89001 & 37.8901 \\
\hline & & & 89.4500 \\
\hline & & & 37.7501 \\
\hline & & & 37.7800 \\
\hline & & 37.80001 & $37.8,000 \times 001$ \\
\hline & & 37.80002 & $37.8,000 \times 002$ \\
\hline & & & 37.8001 \\
\hline & & & 37.7701 \\
\hline & & & 37.7600 \\
\hline & & 37.78001 & \\
\hline \multirow[t]{8}{*}{ Implantable defibrillator / cardiac resynchronization therapy defibrillator } & & & Z95.800x007 \\
\hline & & & Z45.800x006 \\
\hline & & & T82.100x011 \\
\hline & & & T82.100x010 \\
\hline & & & 00.5100 \\
\hline & & 00.51001 & $00.5,100 \times 001$ \\
\hline & & & 00.5101 \\
\hline & & & 00.5102 \\
\hline
\end{tabular}




\section{Appendix 6| (Continued) Coding of CVD operations}

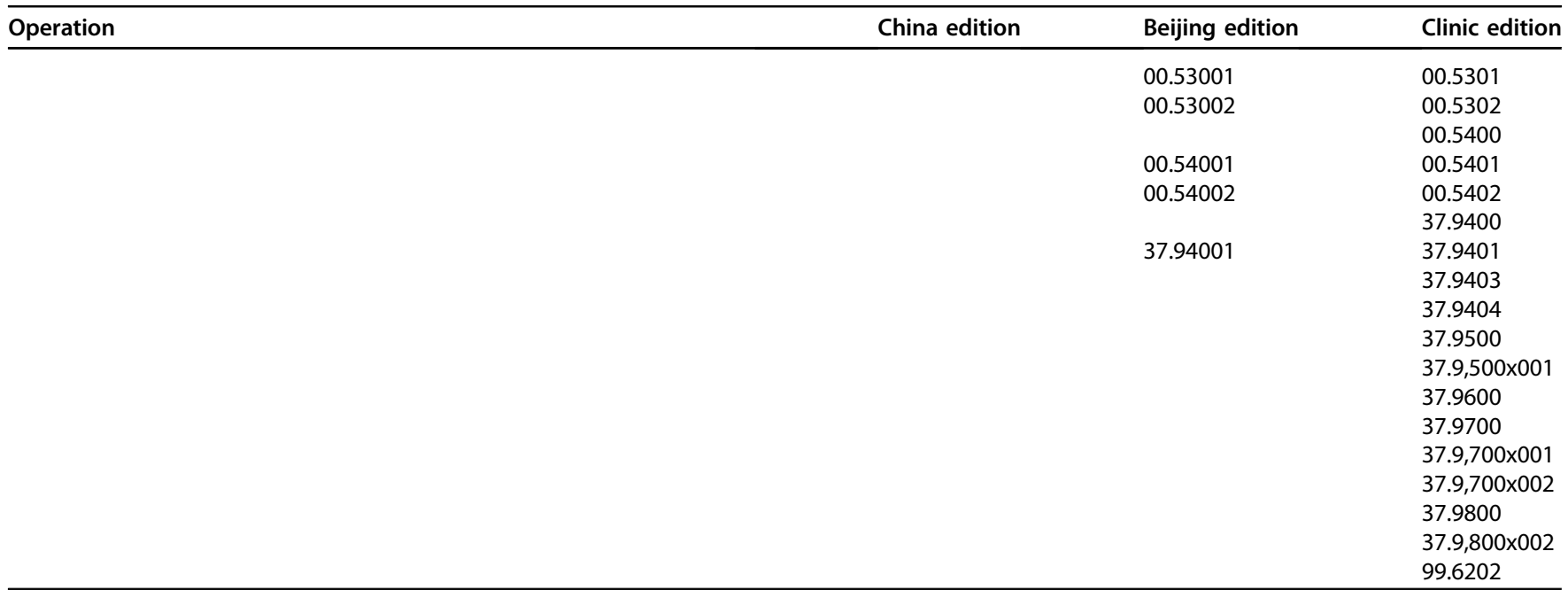

CVD, cardiovascular disease.

\section{Appendix 7| Coding of AKI}

\begin{tabular}{|c|c|c|c|c|}
\hline AKI & All editions & China edition & Beijing edition & Clinic edition \\
\hline Acute renal failure & N17 & & & \\
\hline Rapidly progressive nephritic syndrome & N01 & & & \\
\hline Traumatic anuria & T79.5 & & & \\
\hline Hemolytic-uremic syndrome & D59.3 & & & \\
\hline Hepatorenal syndrome & K76.7 & & & \\
\hline Postpartum acute renal failure & O90.4 & & & \\
\hline Renal failure after abortion & O08.4 & & & \\
\hline Postprocedural disorders of genitourinary system, not elsewhere classified & N99.0 & & & \\
\hline Acute tubulointerstitial nephritis & & N10.x00 & & $\mathrm{N} 10 . \mathrm{x} 00$ \\
\hline Acute interstitial nephritis & & N10.x01 & & N10.x01 \\
\hline Chronic glomerulonephritis with rapidly progressive glomerulonephritis & & & N00.908 & N00.900x009 \\
\hline Acute Infectious interstitial nephritis & & & N10xx03 & N10.x00x003 \\
\hline TINU syndrome & & & $\mathrm{N} 10 \times x 04+\mathrm{H} 20.9^{\mathrm{a}}$ & $\mathrm{N} 12 . \times 00 \times 005$ \\
\hline
\end{tabular}

AKI, acute kidney injury; TINU, tubulointerstitial nephritis and uveitis.

${ }^{\mathrm{a}}$ Denotes to any possible number so that the whole sequence represents all the subclasses of the disease. 